\title{
X-RAY SCATTERING AT THE PERIPHERY: FORBIDDEN BRAGG REFLECTIONS AND SILICON NANOWIRE ARRAYS
}

\author{
A Dissertation presented to \\ the Doctoral Committee \\ at the University of Missouri
}

\author{
In Partial Fulfillment \\ of the Requirements for the Degree \\ Doctor of Philosophy
}

by

JESSE WADE KREMENAK

Dr. Paul F. Miceli, Dissertation Supervisor

MAY 2018 
(c) Copyright by Jesse Wade Kremenak 2018

All Rights Reserved 
The undersigned, appointed by the Dean of the Graduate School, have examined the dissertation entitled:

\section{X-RAY SCATTERING AT THE PERIPHERY: FORBIDDEN BRAGG REFLECTIONS \\ AND SILICON NANOWIRE ARRAYS}

presented by Jesse Wade Kremenak, a candidate for the degree of Doctor of Philosophy and hereby certify that, in their opinion, it is worthy of acceptance.

Dr. Paul F. Miceli

Dr. Ioan Kosztin

Dr. Gavin King

Dr. C. Michael Greenlief 
To my wife, Danielle 


\section{ACKNOWLEDGMENTS}

First of all, I would like to express my appreciation to my doctoral advisor, Pro-

fessor Paul F. Miceli, for the opportunity to work with him and for his guidance and support.

I would like to thank Christopher J. Arendse and Franscious R. Cummings, both from the University of Western Cape, South Africa, for the opportunity to collaborate.

I am thankful to my Miceli laboratory colleagues, Yiyao Chen, Alexander Daykin, Michael Gramlich, Shawn Hayden, and Alessandro Mazza, with whom I have had the pleasure of working.

Lastly, but not least, I am grateful to my family as well as to all of whom have supported me and who are not listed here - the names are too many to list - grazie mille! 


\section{TABLE OF CONTENTS}

ACKNOWLEDGMENTS .................. i i

LIST OF TABLES .................... vii

LIST OF FIGURES ................... viii

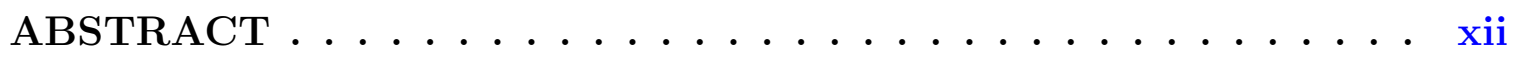

\section{CHAPTER}

1 Introduction ...................... 1

I Silicon Nanowire Arrays . . . . . . . . . . . . 4

2 Insight on the Silver Catalyst Distribution During Silicon Nanowire Array Formation: an X-ray Reflectivity Study . . . . . . . 5

2.1 Introduction . . . . . . . . . . . . . . . 5

2.2 Background .......................... 8

2.3 Experimental .......................... 11

2.3.1 Sample Preparationt . . . . . . . . . . . . . . 11

2.3.2 Electron Microscopy Measurements $\dagger$. . . . . . . . . . . . 12

2.3.3 X-ray Scattering Measurements . . . . . . . . . . . 13

2.4 Results............................... 13

2.4.1 Electron Microscopy† . . . . . . . . . . . . 13

2.4.2 X-ray Diffraction ................... 18

2.4.3 Extracting the X-ray Specular Reflectivity Data . . . . . . . 20

2.4.4 Analysis of the X-ray Specular Reflectivity . . . . . . . . 28

2.5 Discussion . . . . . . . . . . . . . . . . . . . . . . 39 
2.6 Acknowledgments . . . . . . . . . . . . . . . . . . . . . . . 47

\section{Forbidden Bragg Reflections and Charge Density Dis-} tributions in Crystal Truncation Rods (CTRs) . . . . . 49

3 Introduction: Part II . . . . . . . . . . . . . . 50

4 Charge Density Distribution in Real-Space . . . . . . . . . . . 53

4.1 Introduction . . . . . . . . . . . . . . . 53

4.2 Charge Density Distribution . . . . . . . . . . . . . . 54

4.2.1 General Non-spherical Charge Density Distribution . . . . . 54

4.2.2 Diamond Crystal Structure _ . . . . . . . . . . . . 54

5 Charge Density Distribution in Reciprocal-Space . . . . . . . . 59

5.1 Introduction . . . . . . . . . . . . . . . . . 59

5.2 Dawson Formalism . . . . . . . . . . . . . . . . . 60

5.2 .1 Atomic Form Factor $\ldots \ldots$. . . . . . . . . . 60

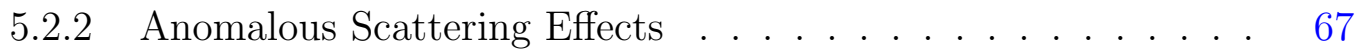

5.2 .3 Structure Factor $\ldots \ldots \ldots \ldots$

5.2.4 Temperature Dependent Structure Factor . . . . . . . . . 70

5.2.5 Anharmonic Temperature Factor: One-Particle Potential model 71

5.3 Scattering From the Diamond Crystal Structure . . . . . . . . . 73

5.3.1 Face-Centered Cubic Structure . . . . . . . . . . . . . 74

5.3.2 Diamond Crystal Structure _. . . . . . . . . . . . 75

5.4 Gamma-Formalism . . . . . . . . . . . . . . . . . . 77

5.4.1 General Charge Density Distribution Correction Factor . . . . 77

5.4.2 Non-spherical Charge Density Distribution Correction for Silicon 80

5.5 Hexagonal Coordinates . . . . . . . . . . . . . . . . . . 90 


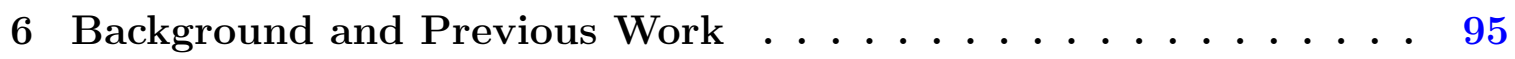

6.1 Introduction . . . . . . . . . . . . . . 95

6.2 Electron Density Distribution from X-ray Scattering . . . . . . . . 97

6.3 Early Forbidden Bragg Reflection Studies by Batterman et al. and Related Work . . . . . . . . . . . . . . . . . 97

6.4 Renewed Interest and Recent Progress _. . . . . . . . . . . 105

7 Forbidden Bragg Reflections and Charge Density Distributions in X-ray CTRs . . . . . . . . . . . . . . . . 107

7.1 Introduction . . . . . . . . . . . . . . . 107

7.2 Crystal Truncation Rods (CTRs) _ . . . . . . . . . . . . 108

7.2.1 $(111)_{C}$ Terminated Diamond Crystal Structure CTR $\ldots \ldots 110$

7.2.2 Specular Reflectivity with Spherical Charge Density Distribution112

7.2.3 Specular Reflectivity with Non-Spherical Charge Density Distribution . . . . . . . . . . . . . . . . . . 114

7.2 .4 In-Plane CTRs . . . . . . . . . . . . . . . . . 117

7.3 Experimental . . . . . . . . . . . . . . . . . . 120

7.4 Results . . . . . . . . . . . . . . . . . . . . . 122

7.4.1 Structure Models . . . . . . . . . . . . . . . . . . . 123

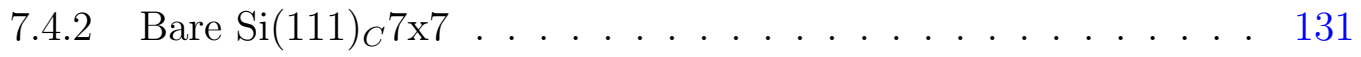

7.4.3 Thin Ag Film on $\operatorname{Si}(111)_{C} 7 \mathrm{x} 7 \ldots \ldots \ldots \ldots$

7.4.4 Thick Ag Film on $\operatorname{Si}(111)_{C} 7 \times 7 \ldots \ldots \ldots \ldots$

7.5 Discussion . . . . . . . . . . . . . . . . . . . . . . . . . . 149 APPENDIX

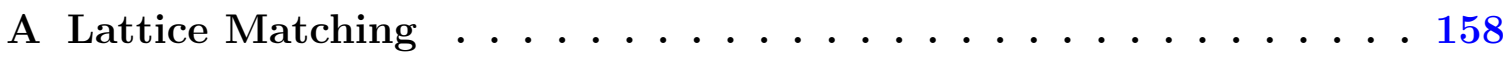

A.1 Lattice Matching . . . . . . . . . . . . . . . 158 
A.2 Potential Film Candidates on $\mathrm{Si}(111)_{C} \ldots \ldots . \ldots 166$ BIBLIOGRAPHY .................. 167

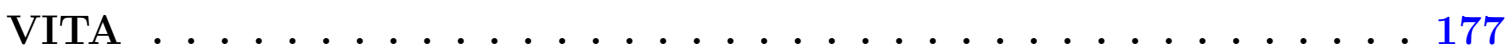




\section{LIST OF TABLES}

Table $\quad$ Page

2.1 Summary of the best-fit parameter values determined from X-ray specular reflectivity .............................. 41

4.1 Charge density distribution parameters . . . . . . . . . . . 57

5.1 Angular factors for the diamond crystal structure . . . . . . . . . 66

5.2 Temperature factor parameters . . . . . . . . . . . . . 72

7.1 Comparison of structure factor and $\gamma$ values for $\operatorname{Si}(222)_{C} \ldots \ldots$. . . 136

7.2 Si charge density distribution parameter values . . . . . . . . . 138

A.1 $(111)_{C}$ Interplanar spacing and the location of $(n n n)_{C}$ Bragg peaks with respect to $\mathrm{Si}(111)_{C}$ reciprocal lattice units. . . . . . . . . . . . . 160

A.2 Potential film candidates on $\mathrm{Si}(111)_{C} \ldots \ldots \ldots 6$ 


\section{LIST OF FIGURES}

$\begin{array}{ll}\text { Figure } & \text { Page }\end{array}$

2.1 Cover artwork for the journal Nanoscale . . . . . . . . . . . . . . 7

2.2 SEM micrograph of the 0 s sample . . . . . . . . . . . . . . . . 14

2.3 SEM micrographs of the 90s, 5m, 15m, and 30m samples . . . . . 15

2.4 TEM micrographs . . . . . . . . . . . . . . . . . . 17

2.5 X-ray diffraction of $\mathrm{Ag}(111) \quad \ldots \ldots \ldots$

2.6 Transverse line shapes for the 90 s sample . . . . . . . . . . . . . . 22

2.7 Intensity maps versus $\omega$ and $20 \ldots \ldots$. . . . . . . . . . 25

2.8 Three-dimentional intensity plots versus $\omega$ and $2 \theta \ldots . . . . . .26$

2.9 Illustration of the method to determine the specular component from a transverse scan measurement . . . . . . . . . . . . . . 27

2.10 X-ray specular reflectivity data for the bare Si, 0s, 90s, and $5 \mathrm{~m}$ samples 29

2.11 Fitted curves to the specular X-ray reflectivity data for the 0s sample 31

$2.12 Q_{c}^{2}$ and composition profiles for the 0 s sample . . . . . . . . 33

2.13 Specular X-ray reflectivity data for the 90s sample with a best-fit curve 35

$2.14 Q_{c}^{2}$ and composition profiles for the 90 s sample . . . . . . . . 37

2.15 Specular X-ray reflectivity data for the $5 \mathrm{~m}$ sample with fitted curves . 38

$2.16 Q_{c}^{2}$ and composition profiles for the $5 \mathrm{~m}$ sample . . . . . . . . . . 40

$2.17 Q_{c}^{2}$ values determined from Yoneda scattering and specular reflectivity 41 
2.18 The effective Ag thickness, $\mathrm{t}^{A g}$, determined from specular X-ray reflec-

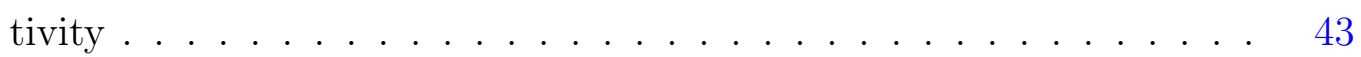

2.19 Etch thickness determined from SEM and X-ray specular reflectivity . 45

4.1 Schematic diagram of the diamond crystal structure cubic unit cell and the bonding configurations of the A and B basis sites. . . . . . . 55

4.2 Three-dimensional plots of the non-spherical components of the electron density distribution . . . . . . . . . . . . . . 58

$5.13^{r d}, 4^{\text {th }}$, and $6^{\text {th }}$ order Fourier-Bessel transform . . . . . . . . 63

5.2 Three-dimensional plots of angular factors of the non-spherical atomic form factor components . . . . . . . . . . . . . 65

5.3 Three-dimensional plots of the non-spherical atomic form factor com-

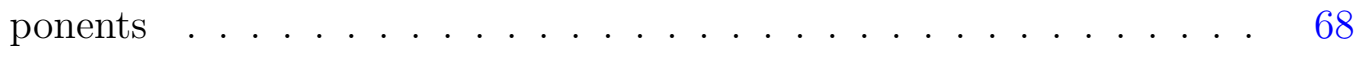

5.4 Cubic diamond-structure lattice and face-centered cubic (FCC) lattice 75

5.5 Three-dimensional plots of $\gamma^{S i}(h k l) \ldots \ldots$. . . . . . . . . 82

$5.6 \operatorname{Im}\left(\gamma^{S i}(h k l)\right)$ along certain crystallographic directions . . . . . . . . 83

5.7 Three-dimensional plots of $\Delta \gamma^{S i}(h k l)$ between $100 \mathrm{~K}$ and $900 \mathrm{~K} \ldots . . \quad 85$

5.8 Temperature dependence of $\operatorname{Im}\left(\gamma^{S i}(h k l)\right)$ for $\operatorname{Si}(222), \operatorname{Si}(442)$, and $\operatorname{Si}(622) 86$

5.9 Temperature dependence of $\operatorname{Im}\left(\gamma^{S i}(442)\right)$ and $\operatorname{Im}\left(\gamma^{S i}(44 \overline{2})\right) \ldots . . \quad 87$

5.10 Three dimensional bubble plots of $\operatorname{Im}\left(\gamma^{S i}(h k l)\right)$ at select forbidden

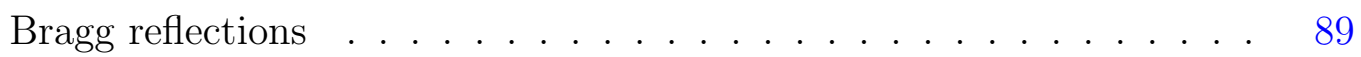

5.11 Three dimensional bubble plots showing the sign of $\operatorname{Im}\left(\gamma^{S i}(h k l)\right)$ at select forbidden Bragg reflections . . . . . . . . . . . . . . . 91

5.12 Schematic of the (111) plane in FCC and a cross-sectional view of the

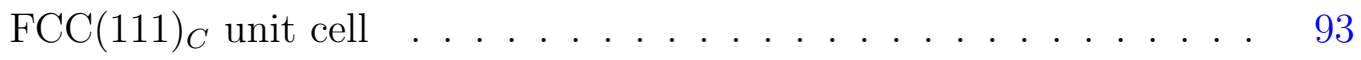

5.13 The diamond crystal structure $(111)_{C}$ lattice vectors . . . . . . . . 94 
6.1 Temperature dependence of the $\mathrm{Si}(442)_{C}$ forbidden Bragg reflection . 101

6.2 Charge anisotropy directions in the tetrahedral bonding configuration 102

7.1 Best-fits of the spherical charge density distribution model to the specular X-ray reflectivity data of bare $\mathrm{Si}(111)_{C} 7 \times 7$ and thin $\mathrm{Ag}$ film on $\operatorname{Si}(111)_{C} 7 \mathrm{x} 7 \ldots \ldots \ldots \ldots$

7.2 Calculated specular X-ray reflectivity of the $(111)_{C}$ diamond crystal structure and its components . . . . . . . . . . . . 113

7.3 Calculated specular X-ray reflectivity near the diamond crystal structure $(222)_{C}$ forbidden Bragg reflection . . . . . . . . . . . . 116

7.4 Calculated in-plane CTRs of the $\mathrm{Si}(111)_{C}$ crystal structure . . . . . . 118

7.5 Maps of forbidden Bragg reflections in reciprocal-space . . . . . . . . 121

7.6 Best-fits to X-ray specular reflectivity data of bare $\operatorname{Si}(111)_{C} 7 x 7$, thin Ag film on $\mathrm{Si}(111)_{C} 7 \times 7$, and thick $\mathrm{Ag}$ film on $\mathrm{Si}(111)_{C} 7 \times 7 \ldots 124$

7.7 Best-fits to X-ray specular reflectivity data of bare $\operatorname{Si}(111)_{C} 7 \times 7$. . 132

$7.8 \mathrm{Si}(222)_{C}$ forbidden Bragg reflection characteristic edge . . . . . . . 135

7.9 Real-space electron density distribution . . . . . . . . . . . . . . 139

7.10 Fits to the X-ray specular reflectivity of thin $\mathrm{Ag}$ film on $\mathrm{Si}(111)_{C} 7 \mathrm{x} 7 . \quad 141$

7.11 Comparing fits to the X-ray specular reflectivity of thin Ag film on $\operatorname{Si}(111)_{C} 7 \mathrm{x} 7 \ldots \ldots \ldots \ldots \ldots$

7.12 Comparing fits to the X-ray specular reflectivity of thin Ag film on $\operatorname{Si}(111)_{C} 7 \mathrm{x} 7 \ldots \ldots \ldots \ldots \ldots$

7.13 Fits to the X-ray specular reflectivity of thick Ag film on $\mathrm{Si}(111)_{C} 7 \times 7.148$

7.14 Calculated X-ray specular reflectivity curves of different Ag film thick-

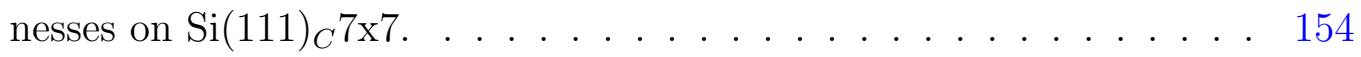


7.15 Calculated X-ray specular reflectivity curves of thick Ag film on $\operatorname{Si}(111)_{C} 7 x 7$ with different Si substrate roughness. . . . . . . . . . . 155 


\begin{abstract}
In this work, we developed two separate unconventional X-ray scattering techniques that enable us to extract novel information from distinct systems at disparate length scales. We examine the formation of Si nanowire (SiNW) arrays during Ag metal-assisted chemical etching (MACE). The exceptionally rough surface of these nanowire arrays result in very low optical reflectivity, which also makes it extremely challenging to measure the X-ray specular reflection. Nevertheless, we demonstrate that with proper care, X-ray specular reflection can be measured and utilized to obtain unique structural information about the composition profile of both $\mathrm{Ag}$ and $\mathrm{Si}$ during the formation of the SiNWs. Secondly, we have discovered a novel interference effect between the crystal surface and the bulk forbidden Bragg reflection that allows — for the first time — both the amplitude and phase of the forbidden reflection to be resolved. The newly attained amplitude and phase information permits one to extract the structure of the non-spherical electron density distribution from diamond crystal structures. We employed X-ray specular reflectivity, a technique conventionally used to study interfaces and surfaces, to examine the non-spherical electron density distribution of bulk Si. We have discovered and demonstrated that the structure of the covalent bond can be ascertained from the weak scattering between the Bragg reflections along the crystal truncation rod. This discovery significantly expands the experimental capability of examining the structure of electron densities, and especially the valence electrons in crystals. Using these novel and unconventional X-ray scattering techniques, we were able to extract valuable information about each system from exceptionally faint signals.
\end{abstract}




\section{Chapter 1}

\section{Introduction}

In this work unconventional X-ray scattering techniques were developed and used to study two distinct systems at disparate length scales. In both investigations we examine Si substrates with and without Ag nanoparticles on the surface. The size of the stuctures examined however, are quite different between the two systems. We were able to investigate the formation of Si nanowire (SiNW) arrays that are hundreds of nanometers tall as well as, in other samples, examine the fine details of the electron density distribution around an atom - either task could be a superlative because these length scales are at the peripheries of the dynamic range of X-ray scattering. Furthermore, using novel experimental techniques, we were able to extract valuable information about each system from exceptionally weak signals.

In one system, $\mathrm{Si}$ nanowire (SiNW) arrays, which are of interest for a variety of technological applications, [1-10] are fabricated by the metal-assisted chemical etching (MACE) method. In this method Ag nanoparticles migrate deep into the semiconductor, forming uniform diameter crystalline nanowires and results in a remarkably rough surface. However, the mechanisms by which MACE forms these structures is poorly 
understood. Prior studies have observed the role of initial nanoparticle morphology deposited on the $\mathrm{Si}$ surface impacts the lateral geometry of the nanowires,[11, 12] yet the vertical distribution of the buried metal nanoparticles has not been carefully studied even though it should be critical to the process that drives the anisotropic etching. Utilizing X-ray specular reflectivity we have revealed fundamentally new information about the evolution of the metal-catalyst distribution, including its flow in and out of the nanowire array, as well as the size and coarsening of the nanoparticles, as a function of etch duration.

In the second system, the substrate surface is exceptionally smooth, which as we have discovered allows us to extract critical information about the electron density distribution of non-centrosymmetric atoms from the bulk crystal. Prior studies[1318] relied on a combination of challenging temperature dependent X-ray and neutron diffraction measurements to determine the phase information about the non-spherical charge density distribution, which is necessary to resolve the structure of the electron density distribution. Whereas we have discovered a novel interference effect between the surface waves and the bulk forbidden Bragg reflection that allows us to ascertain critical phase information from a single X-ray crystal truncation rod measurement. The newly attained phase information also permits us to extract the structure of the covalent bond in Si from extended range specular X-ray reflectivity. By comparison, diffraction techniques are restricted to discerning charge density distribution from a limited number of Bragg peaks. Whereas we have utilized the crystal surface to extract the structure of the bulk electron density distribution from the scattering between the Bragg peaks.

This text is organized into two parts that reflect the two system that were examined. Part I contains the X-ray reflectivity study of the silver catalyst distribution during silicon nanowire array formation. Part II contains the work on forbidden Bragg 
reflections and charge density distributions in crystal truncation rods.

Part II is organized into several chapters: In Chapters 4 and 5 we discuss the real-space charge density distribution and reciprocal-space structure of the diamond crystal structure, respectively. Chapter 5 includes the formalism of the atomic form factor, structure factor, and the aspherical charge density distribution correction. Chapter 6 reviews prior work on experimentally determined non-spherical charge density distribution with a focus on forbidden Bragg reflections. In Chapter 7 we discuss the determination of non-spherical charge density distributions in diamond crystal structures from forbidden Bragg reflections in X-ray crystal truncation rods. 


\section{Part I}

\section{Silicon Nanowire Arrays}




\section{Chapter 2}

\section{Insight on the Silver Catalyst Distribution During Silicon Nanowire Array Formation: an X-ray Reflectivity Study}

\section{$2.1 \quad$ Introduction}

Although metal-catalysts are commonly used to create nanoscale materials at surfaces, little is quantitatively known or understood about the depth distribution profile of the catalyst during the growth process. Using X-ray reflectivity, we report the first quantitative investigation, with nanoscale resolution, of the Ag metal-catalyst depth distribution profile during metal-assisted chemical etch growth of Si nanowire (SiNW) arrays on $\mathrm{Si}(100)$. Given the very low optical reflectivity of these nanowire arrays, specular reflection from these materials in the X-ray region is extremely challenging to measure because it probes interfaces on the nanoscale. Nevertheless, we demonstrate that with suitable investigation, X-ray specular reflection can be measured and 
utilized to obtain unique structural information about the composition profile of both $\mathrm{Ag}$ and Si. The measurements, which also include X-ray diffraction and complementary electron microscopy, reveal that the Ag nanoparticles distribute along the length of the nanowires upon etching with a $\mathrm{Ag}$ density that increases towards the etch front. The Ag nanoparticles coarsen with etch time, indicating a high mobility of Ag ions even though we also find that the Ag does not migrate from the SiNW region into the etch bath during etching. The Ag density gradient and the Ag mobility suggest the existence of a strong chemical force that attracts Ag towards the etch front. These results provide unique and important new insight into the growth process for creating SiNWs from wet chemical etching using metal-catalysts.

This chapter has been adapted from Ref. [19] with permission from The Royal Society of Chemistry. This scientific work is the result of a collaboration between the Paul F. Miceli and Christopher J. Arendse laboratories. Arendse and Franscious R. Cummings, both from the University of Western Cape, South Africa, fabricated the samples at the University of Missouri. The electron microscopy measurements were performed by Cummings at the University of Wester Cape. The microscopy analysis was carried out by Arendse and Cummings. Sections 2.3.1, 2.3.2, 2.4.1 are attributed to Arendse and Cummings. The X-ray measurements and analysis was performed by Jesse W. Kremenak.

The cover artwork[20], seen in Fig. 2.1, that showcases this work was published along with Ref. [19]. The cover artwork is the result of a collaboration between Danielle V. Langdon (Columbia College, Columbia, MO) and Kremenak. 


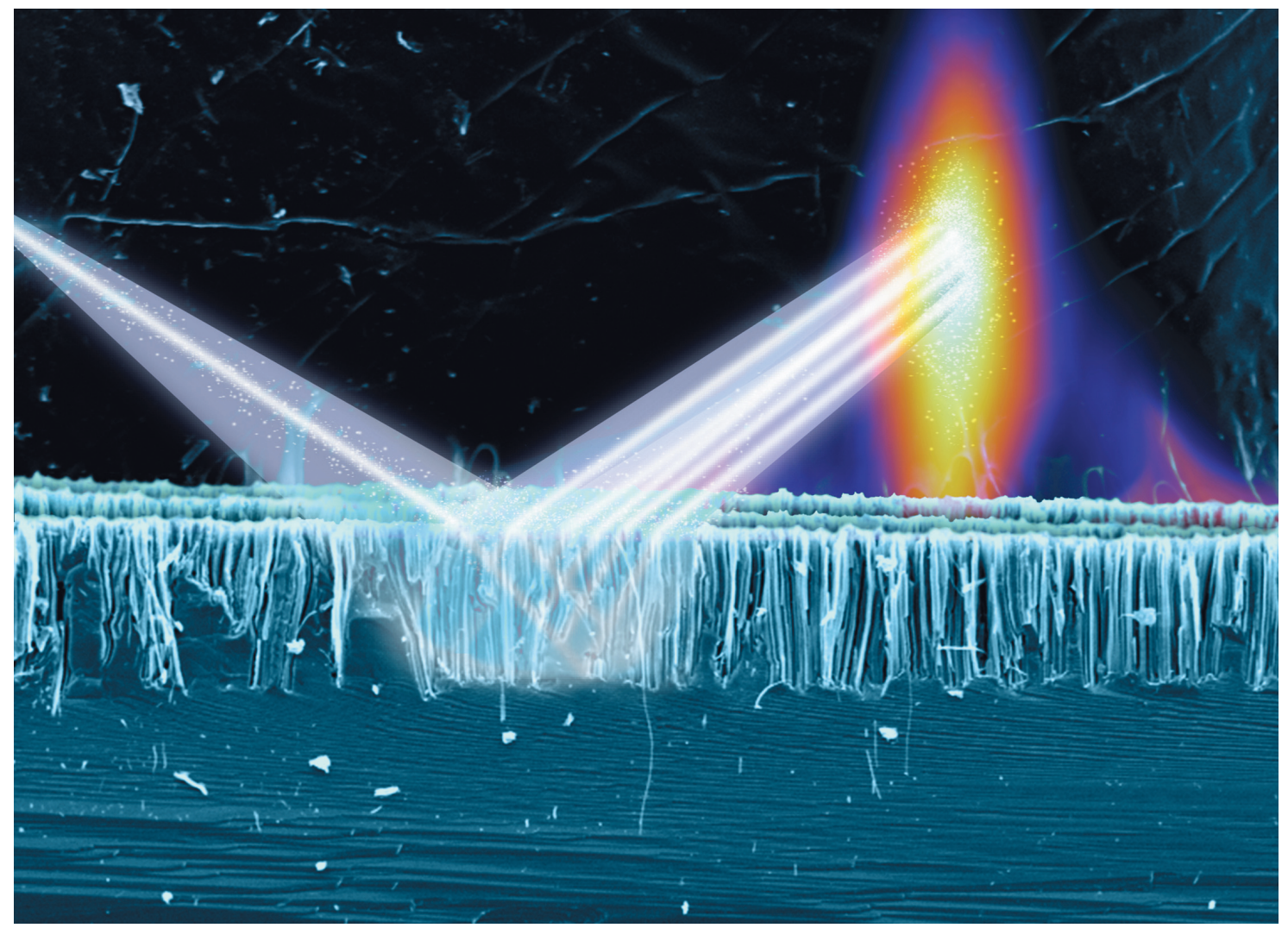

Showcasing research from the Miceli (University of Missouri, USA) and Arendse (University of the Western Cape, ZA) laboratories.

Insight on the silver catalyst distribution during silicon nanowire array formation: an X-ray reflectivity study

The specular reflection of X-rays from Si nanowire (SiNW) arrays is unique in being able to extract the compositional depth profile of the Ag nanoparticle catalysts that participate in the etch formation of the SiNWs. It was revealed that Ag nanoparticles unexpectedly distribute along the length of the nanowires during etching, which provides important insight into the poorly understood metal-assisted chemical etching process. Danielle V. Langdon, Columbia College, and Jesse W. Kremenak, University of Missouri, both in Columbia, MO (USA), are acknowledged for their cover artwork.
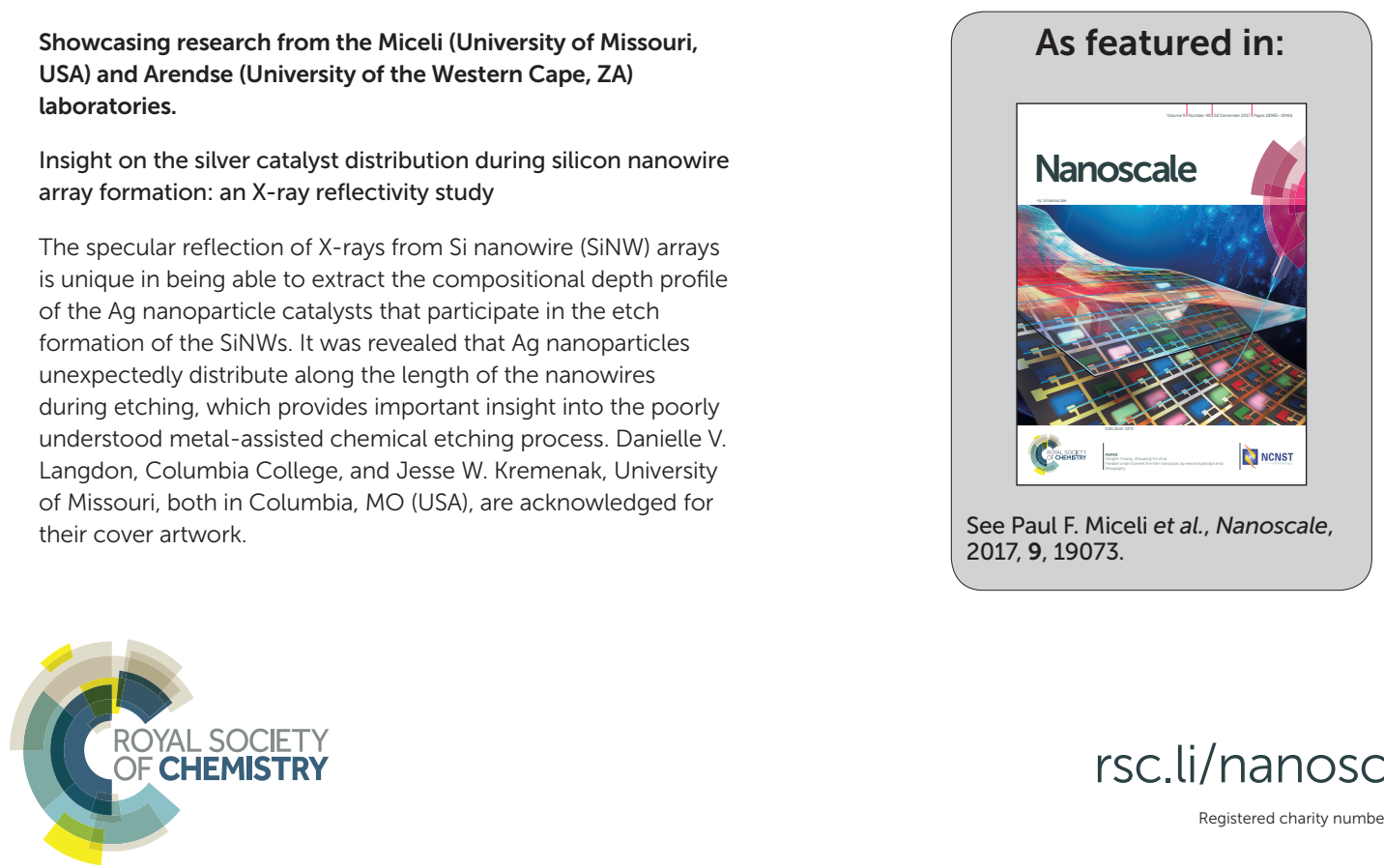

Figure 2.1: Cover artwork for the journal Nanoscale showcasing this research. Reproduced from Ref. [20] with permission from The Royal Society of Chemistry. 


\subsection{Background}

Metal-assisted chemical etching (MACE) is often used to fabricate semiconductor nanostructures because of its easy application, low cost and scalability. MACE involves top-down etching of a semiconductor that is initially coated with metal nanoparticles in a chemical solution.[12] The metal-catalyst nanoparticles aid the etching at the surface, which results in the selective dissolution of the semiconductor and the subsequent migration of the nanoparticles into the etch pit. Nano-pores,[21$23]$-pyramids, [24] -trenches, [25] -cones, [25] and -wires[21, 24-26] are a few examples of the myriad of nanoscale textures and structures that can be fabricated using MACE. Despite the wide application of this fabrication method, there is a very limited fundamental understanding of the role of the metal nanoparticles and the resulting nanoscale geometries.[12]

Nanowire arrays fabricated by MACE, which are of interest for a variety of technological applications, are particularly noteworthy because uniform diameter nanowires are formed by the deep migration of the metal-catalyst into the semiconductor. The mechanism by which this process occurs is poorly understood. Prior studies have observed that the initial morphology of metal deposited on the semiconductor surface plays a role in the lateral geometry of the nanowires.[11, 12] However, the vertical (depth) distribution of the buried metal has not been carefully studied, despite the fact that it should be central to the process of catalysis that drives the anisotropic etching of the semiconductor to form the nanowires. The reason for the absence of such studies is due, in part, to the difficulty in finding a suitable technique to determine the vertical metal distribution. A variety of experimental techniques have previously been used to examine nanowire arrays fabricated by MACE, including elec-

tron microscopy, atomic force microscopy, Raman spectroscopy, X-ray photoelectron 
spectroscopy, and X-ray diffraction; however, these tools are limited in their ability to determine a depth composition profile.[27]

In this paper, we introduce X-ray specular reflectivity (XRR) as a technique to characterize the amount and distribution of the metal-catalyst during the formation of nanowires. Our investigation reveals fundamentally new information about the evolution of the metal-catalyst distribution, its flow in and out of the nanowires, as well as the size and coarsening of the metal nanoparticles, as a function of the etch time. XRR is unique in its ability to quantitatively probe the vertical subsurface composition profile on the nanoscale. Although it is frequently used to study relatively sharp buried interfaces of epitaxial materials,[28] there are significant challenges to using XRR methods to study nanowire arrays because of the extreme roughness of these surfaces. Nevertheless, we show how the specular reflection can be extracted from the data, which contains detrimentally strong diffuse scattering, so that useful information about the metal-catalyst distribution as a function of etch time can be obtained.

Our studies were performed on $\mathrm{Si}(100)$ nanowire (SiNW) arrays using a Ag catalyst. SiNWs have attracted considerable interest for a broad variety of applications due to their unique structural, electrical, optical, and thermoelectrical properties, which are different from those of bulk Si. [4, 7] Advanced electronic devices, $[1,2]$ opto-electrical devices, [3, 4] high-performance batteries, [5] photovoltaic devices, [4, 6, 7] thermoelectrical devices, $[8,9]$ therapeutic drug delivery,[10] and biosensing[10] are just a few examples of the myriad of novel applications of SiNWs.

Vertically-aligned nanowire arrays can significantly improve the efficiency of photovoltaic cells by reducing the unusable light energy that is reflected from the surface. Laminar antireflective films, which are conventionally employed to reduce the wasted reflected energy, have limitations in their spectral range of operation, thermal mis- 
match, adhesion, and stability.[29] Alternatively, SiNW arrays can be used to reduce inefficient reflection by providing a highly rough surface of the photocells - this approach has been shown to not only dramatically reduce the amount of light that is reflected, but it also provides a much wider operational bandwidth than conventional antireflective coatings. [7, 29]

Several methods have been used to fabricate SiNW arrays. Bottom-up methods grow the material on the substrate using various techniques such as laser ablation, chemical vapor deposition, and oxide-assisted growth. These methods, however, can have costly drawbacks such as requiring high-temperature processing and highvacuum environments.[7, 11] Alternatively, top-down methods remove material by etching, which is low-cost and relatively simple to use.

MACE is an effective top-down method for fabricating vertically-aligned SiNW arrays and is commonly performed in either one or two steps.[11] In the one-step method, a metal-catalyst is deposited onto the $\mathrm{Si}$ wafer while simultaneously etching the surface using a single solution (commonly $\mathrm{AgNO}_{3} / \mathrm{HF}$ ).[30-32] Alternatively, in the two-step method used in this study, the metal deposition and wet chemical etching are performed in separate steps, where the metal-catalyst can be deposited on the Si surface with a variety of techniques including thermal evaporation,[33] sputtering,[11] electrochemical deposition,[34] or by precipitation from solution[35]. The metal-coated Si wafer is then etched in a HF chemical bath that usually includes $\mathrm{H}_{2} \mathrm{O}_{2}$ as an oxidizing agent.[12]

In addition to XRR, our investigation utilizes X-ray diffraction (XRD) and complementary electron microscopy to characterize the amount and distribution of $\mathrm{Ag}$ during the formation of SiNWs. Surprisingly, our results demonstrate that the total amount of $\mathrm{Ag}$ within the nanowire array does not change during the etch process. It is a particularly striking result given that we also observe Ag nanoparticle coars- 
ening, which indicates a significant mobility of the $\mathrm{Ag}$ ions in the solution within the nanowire region. Importantly, these quantitative measurements on the nanoscale establish that the Ag depth distribution is spread along the length of the nanowires, with a Ag density gradient that increases towards the etch front. These findings suggest that there is a force which overcomes the diffusion gradient and attracts $\mathrm{Ag}$ towards the etch front while leaving Ag nanoparticles distributed along the SiNWs. The wires do not exhibit a tapered geometry along their length, indicating that the etching process is extremely anisotropic for the conditions used. Our experimental observations provide new and important insight on models that have been proposed to explain the etching process and, moreover, our results demonstrate how XRR techniques might be used to investigate other surface processing systems that possess a high degree of surface roughness.

In the following, Section 2.3 gives experimental details while Section 2.4 presents our experimental results. Special attention is given to the discussion of the XRR analysis because it is new to the application to SiNW arrays: Section 2.4.3 illustrates how specular reflection data is obtained and extracted whereas Section 2.4.4 explains the analysis of the data. The important new implications of our results is discussed in Section 2.5.

\subsection{Experimental}

\subsubsection{Sample Preparation $\dagger$}

Single-side polished p-type $\operatorname{Si}(100)$ wafers of thickness $\sim 300 \mu \mathrm{m}$ and resistivity of $1-10 \Omega \cdot \mathrm{cm}$ were used as the starting material. Vertically-aligned SiNW arrays were prepared by Ag-assisted wet chemical etching of the $\mathrm{Si}(100)$ substrates at room 
temperature that consisted of the following main steps: 1) The Si wafers were first cleaned by sonicating them in acetone and then in methanol for 15 min each to remove organic contamination. They were then rinsed with deionized water $\left(\mathrm{DI}-\mathrm{H}_{2} \mathrm{O}\right)$. The native oxide $\left(\mathrm{SiO}_{2}\right)$ was then removed by immersing the wafers in a solution of 1.39 M hydrofluoric acid (HF) for $60 \mathrm{sec}$ in a Teflon beaker; 2) Ag-nanoparticles were subsequently deposited on the cleaned Si wafers by dipping them into an aqueous solution of $0.14 \mathrm{M} \mathrm{HF}$ and $0.01 \mathrm{M}$ silver nitrate $\left(\mathrm{AgNO}_{3}\right)$ at room temperature for 30 sec; 3) The Ag-coated Si wafers were then etched by immersing them in a solution of 5.52 M HF and 1.96 M hydrogen peroxide $\left(\mathrm{H}_{2} \mathrm{O}_{2}\right)$ for different time durations. Separate etch baths, in individual Teflon beakers, were used for each etch duration. The etched wafers were then rinsed with DI- $\mathrm{H}_{2} \mathrm{O}$. Six samples were examined. For reference, a bare Si wafer containing only native oxide was examined as well as a substrate having the initially deposited Ag without etching ( "0s"). Four other samples having different etch times of $90 \mathrm{sec}, 5 \mathrm{~min}, 15 \mathrm{~min}$, and $30 \mathrm{~min}$, referred to as "90s", "5m", "15m", and "30m", respectively, were also investigated.

\subsubsection{Electron Microscopy Measurements $\dagger$}

Microscopy measurements were performed at the University of Western Cape, South Africa, using a Zeiss Auriga field emission gun scanning electron microscope (FEG-SEM), operated at electron accelerating voltages of $5 \mathrm{kV}$ for imaging and $20 \mathrm{kV}$ for energy dispersive X-ray spectroscopy (EDS) analysis. The EDS spectra were collected using an Oxford Instruments X-Max silicon solid-state drift detector, with each spectrum collected for $30 \mathrm{sec}$. Transmission electron microscopy analyses were performed using an FEI Tecnai $\mathrm{G}^{2} 20$ FEG-TEM operated at $200 \mathrm{kV}$ and a Fischione high angular annular dark field (HAADF) detector for scanning-TEM (STEM) imaging. 


\subsubsection{X-ray Scattering Measurements}

The X-ray scattering measurements were performed at the University of Missouri using a Rigaku RU-300 18kW rotating anode X-ray generator with a line beam from a Mo target. A Ge(111) monochromator was used in conjunction with a slit to produce a high-resolution beam containing only $\operatorname{MoK} \alpha_{1}$ radiation with a wavelength of $\lambda=0.70926 \AA$. In the scattering plane the beam width was $0.12 \mathrm{~mm}$ with an angular divergence of $0.003^{\circ}$. The angular divergence out of the scattering plane was several degrees. The intensity data from each of the samples was monitor normalized and corrected for footprint and sample area in order to compare intensity across the samples.

\subsection{Results}

\subsubsection{Electron Microscopy $\dagger$}

The surface morphology and composition of the Ag-coated Si substrates and the SiNWs were investigated by scanning electron microscopy (SEM) and energydispersive X-ray spectroscopy (EDS), respectively. Figure 2.2 shows results for the 0s sample where the initial Ag thin film is deposited on the Si substrate. As can be seen from Fig. 2.2(b), the Si surface is modified and forms a faceted morphology upon Ag deposition. The inset of Fig. 2.2(b) shows the spot analysis during EDS to confirm the presence of Ag. At higher magnification, Fig. 2.2(c) reveals that the Ag thin film is composed of an interconnected, dense network of Ag agglomerates, with the average Ag nanoparticle diameter width of $330 \AA$, which vary in size by $\sim \pm 35 \%$ (see Fig. 2.2(d)).

Figure 2.3 shows the evolution of the morphology with etching time. It is noted 


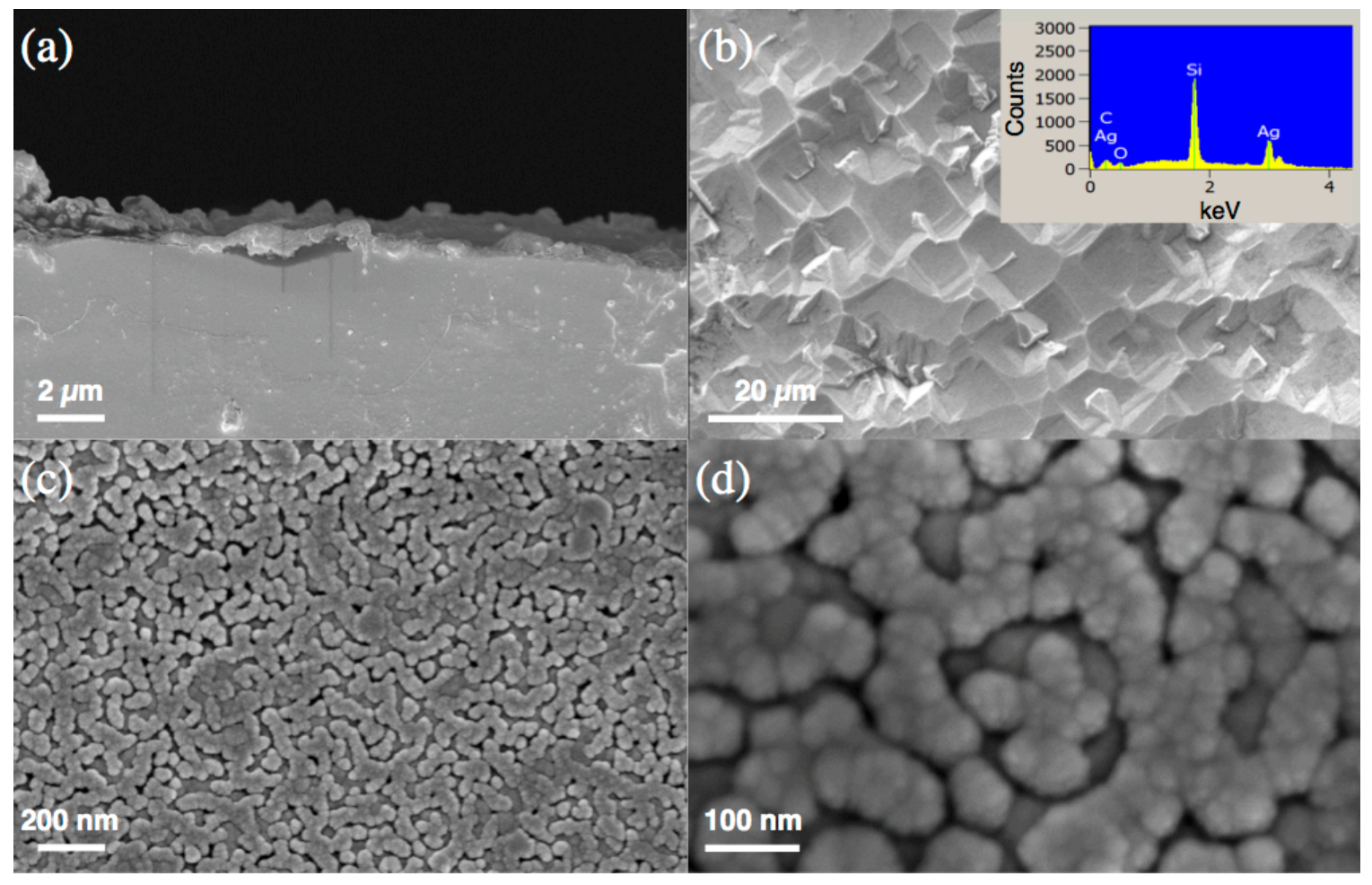

Figure 2.2: (a) cross-sectional and (b) plan view SEM images of the 0s sample. (c) and (d) higher magnified plan view micrographs showing the network of interconnected Ag clusters which are comprised of nano-sized particles. 


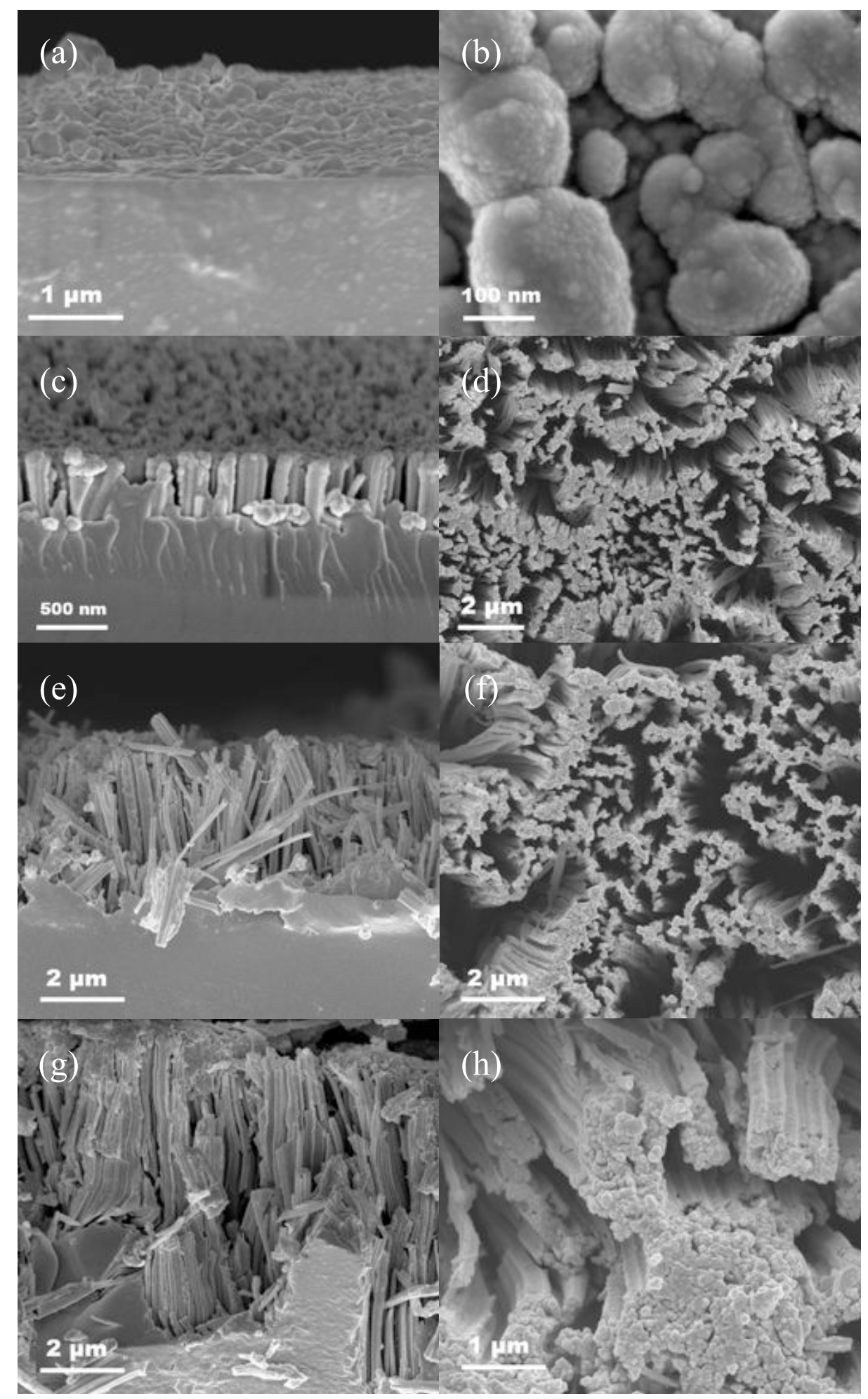

Figure 2.3: Cross-sectional (left) and plan (right) view SEM micrographs of the etched samples: (a, b) 90s; (c, d) 5m; (e, f) 15m; and (g, h) 30m. 
that $90 \mathrm{sec}$ of etching yields no nanowires (NWs) (Fig. 2.3(a)), although, the $\mathrm{Ag}$ nanoparticles are starting to burrow into the Si surface, as shown in in Fig. 2.3(b). This confirms the initial pit formation in the Si substrate via oxide formation by the catalytic action of the Ag particles, followed by its dissolution by the HF. [35, 36] After 5 min of etching, it is evident that SiNW formation is along the [001] direction as seen in Fig. 2.3(c). It has been shown that the $<100\rangle$ is the preferred etching direction when the acid-to-oxidant molar ratio $\left([\mathrm{HF}] /\left[\mathrm{H}_{2} \mathrm{O}_{2}\right]\right)$ is sufficiently low to remove the relatively low number of $\mathrm{Si}$ atoms from the (100) plane.[37] $\mathrm{Ag}$ nanoparticles are seen at the base of the NWs, which is at the etch front, after 5 min of etching in Fig. 2.3(c). EDS analysis confirms Ag at the bottom and detects a low concentration of $\mathrm{Ag}$ on the SiNW walls, which is consistent with the quantitative XRR results discussed below. Tapered or pitted morphologies of the NWs are not observed, which indicates negligible lateral etching.

A wide diameter distribution of $800-2900 \AA$ of the NWs is notable after 5 min of etching and it remains as etching progresses. From the planar views in Fig. 2.3(b, d, f, h), it is evident that the tops of the SiNWs are bundled and the bundling increases as the aspect ratio (length:diameter) increases. The bundling is ascribed to the surface tension forces exerted on the SiNWs during drying.

Figure 2.4 shows transmission electron microscopy (TEM) and selected area electron diffraction images of NWs obtained from the $5 \mathrm{~m}$ sample. The bright-field electron micrograph of Fig. 2.4(a) shows that the NWs have a faceted morphology, rather than being cylindrical. This shape is confirmed by the dark-field scanning-TEM (STEM) micrograph of Fig. 2.4(b) along with the profile of the structure when scanning the electron beam across the width of the NW (Fig. 2.4(b) inset).

The selected area electron diffraction pattern of Fig. 2.4(c), which was collected with the electron beam orientated along the [001] direction, showed no distinct 


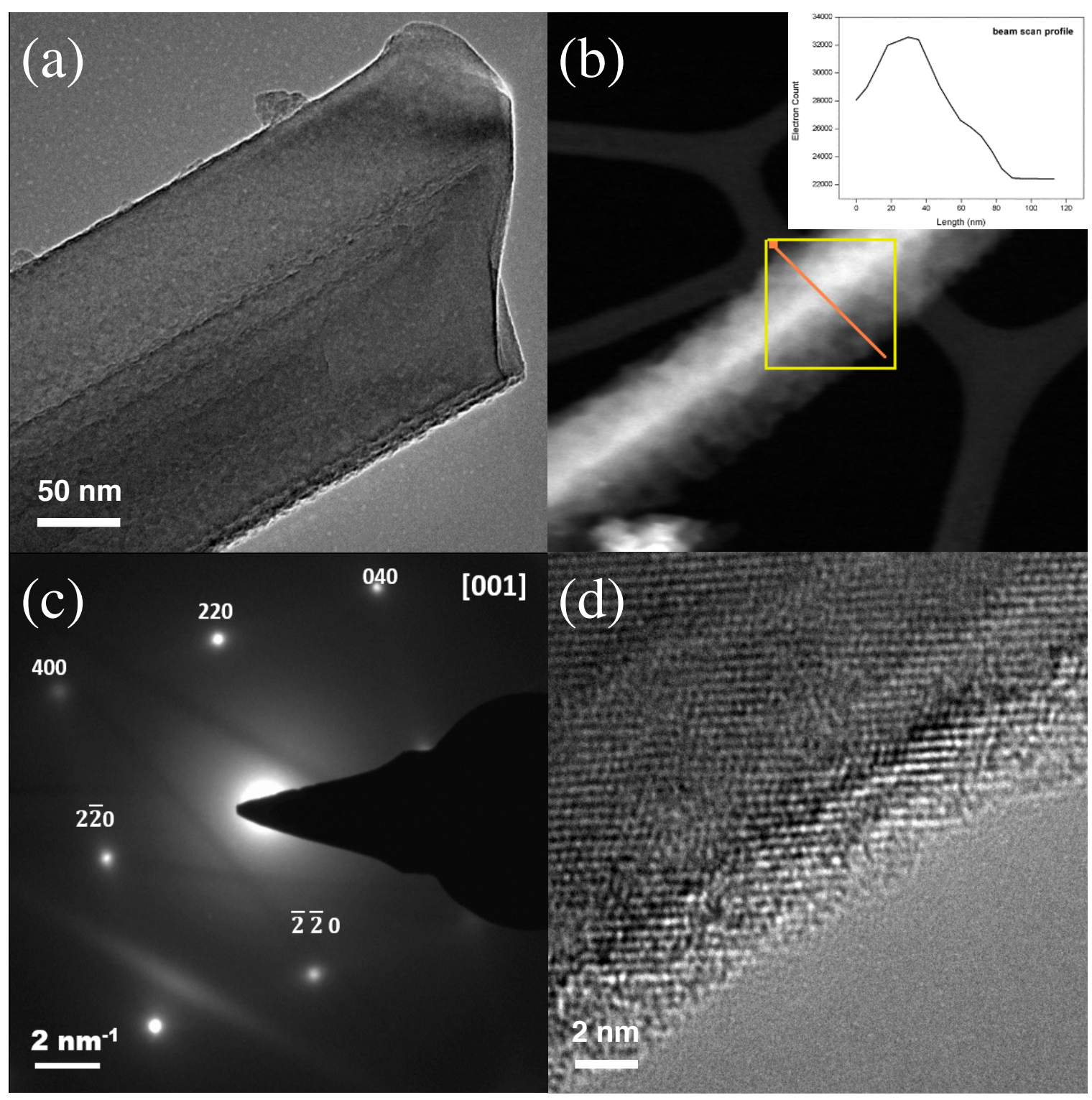

Figure 2.4: (a) Bright-field electron micrograph of a SiNW, (b) STEM micrograph of a NW, which is different than the one shown in (a), with the line profile shown in the inset, obtained from scanning the electron beam across the width of the NW, (c) selected-area electron diffraction, and (d) TEM image of the edge of the crystalline NW. 
changes in the lattice spacing due to strain and shows that the NWs are crystalline. The TEM image of Fig. 2.4(d) shows that the crystallinity of the NWs extends to the edge. Remarkably the disorder on the walls is small in comparison to the diameter of the NW, which indicates negligible etching of the walls.

\subsubsection{X-ray Diffraction}

The $\operatorname{Ag}(111)$ powder diffraction peak was measured, in reflection geometry, to investigate the evolution of the $\mathrm{Ag}$ nanoparticles with etch time. To obtain the integrated intensity, the peak was fit to a Gaussian function and a linear background, as shown in the inset of Fig. 2.5 for the $15 \mathrm{~m}$ sample. Figure 2.5(a) reveals that the integrated intensity is constant with etch time, although, the 90s sample exhibits a lower intensity for reasons that are unknown; it is assumed that the trend is correctly established by the other samples. As discussed below, this trend is also observed by XRR. The constant integrated intensity indicates that the amount of Ag does not change with etch time.

The Ag crystalline grain size, $L$, was determined from the full-width-at-halfmaximum (FWHM), $\Delta 2 \theta$, of the Bragg peak according to the Scherrer equation, [38] $L=\lambda /(\cos \theta \Delta 2 \theta)$, where $\theta$ is the Bragg angle. As seen in Fig. 2.5(b), the Ag grain

size increases with etch time at a rate of $\sim 6 \AA / \mathrm{min}$ and it approximately doubles over the total etch time that was investigated. Conceivably the particle size can be larger than the crystalline grain size if the particles are comprised of multiple grains. Our XRR measurements that are discussed below, however, indicate that the particle size is equal to the grain size because the initial Ag layer thickness for the 0s sample is observed to be equal to the grain size. Given the observed constant integrated intensity in Fig. 2.5(a), which indicates Ag does not leave the sample during etching, the 


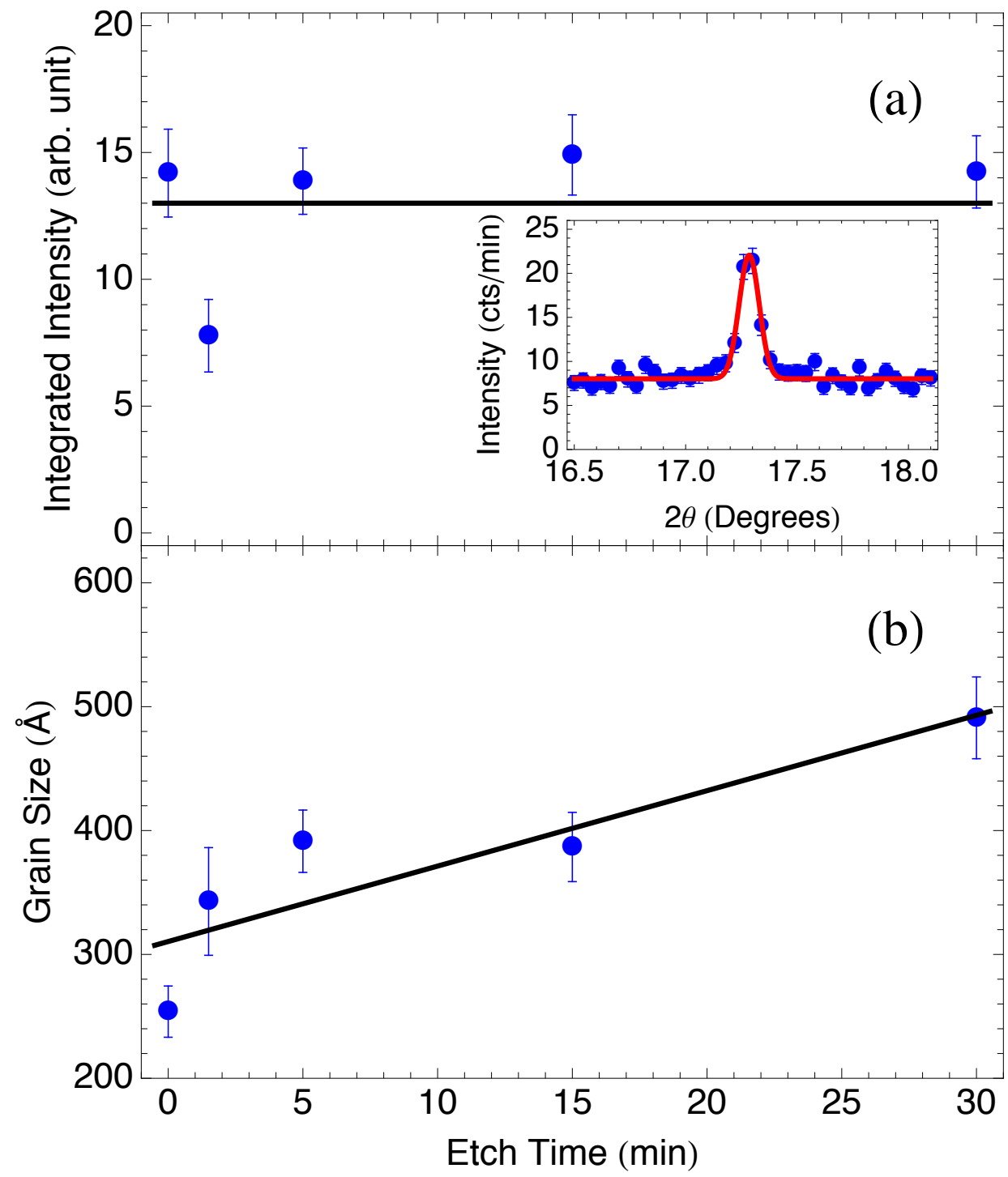

Figure 2.5: (a) integrated intensity and (b) grain size determined from the $\mathrm{Ag}(111)$ Bragg reflection as a function of etch time. The inset in (a) shows the Ag(111) Bragg peak data along with a Gaussian-fitted curve with background for the $15 \mathrm{~m}$ sample. The horizontal line in (a) is the average integrated intensity and the best-fit line in (b) indicates that the $\mathrm{Ag}(111)$ grains have a coarsening rate of $\sim 6 \AA / \mathrm{min}$. 
growing grain size is derived only from the Ag that is initially deposited. Therefore, these results indicate that the $\mathrm{Ag}$ is highly mobile within the sample and it exhibits classic coarsening behavior[39] where smaller grains contribute their material to larger grains over time.

$\mathrm{Si}(111)$ Bragg diffraction was also investigated on the $5 \mathrm{~m}$ and $30 \mathrm{~m}$ samples in order to address the crystallographic orientation of the SiNW arrays. The Si(111) peak was not observed, indicating that the SiNWs do not exhibit the [111] orientation along the surface normal nor are they polycrystalline to within our measurement sensitivity, which is consistent with our electron microscopy results (see Fig. 2.4(c) and (d)) and is in agreement with other studies.[40]

\subsubsection{Extracting the X-ray Specular Reflectivity Data}

$\mathrm{X}$-ray reflectivity is a well-established technique for interrogating the smooth interfaces of thin films.[28, 41, 42] Due to the extremely large surface roughness of the present samples, however, special attention must be given to extracting the specular component of the reflectivity. One problem results from the very limited angular range over which specular reflectivity data can be obtained due to the large roughness. The problem is compounded, however, by other scattering features such as the large diffuse scattering in the vicinity of the specular peak, Yoneda scattering and even the transmitted beam - all interfere with identifying the specular peak and contribute to the difficulty in determining a specular reflectivity curve.

The scattering geometry is shown in the inset of Fig. 2.6, where $\vec{k}_{1}$ and $\vec{k}_{2}$ are the incident and outgoing wavevectors with $\theta_{1}$ and $\theta_{2}$ being the respective angles between these vectors and the sample surface. The scattering angle of the detector is given as $2 \theta=\theta_{1}+\theta_{2} \cdot \vec{Q} \equiv \vec{k}_{2}-\vec{k}_{1}$ is the wavevector transfer and its angle of rotation away 
(transverse direction) from the surface normal direction is given by $\omega=\frac{1}{2}\left(\theta_{1}-\theta_{2}\right)$. These relations also lead to $\theta_{1}=\theta+\omega$ and $\theta_{2}=\theta-\omega$.

The challenge in extracting the specular reflection, which should appear as a peak at $\omega=0$, is illustrated in Fig. 2.6 that shows several transverse line shapes for the 90s sample that were obtained by rocking the sample ( $\omega$ scan) with the detector $(2 \theta)$ fixed. It can be seen that in addition to a central specular peak there are a number of additional scattering features present. Beams that refract and transmit through the surface, rather than reflect, lead to prominently visible peaks labeled "T" in Fig. 2.6. Refraction is given by Snell's law and, in the small angle approximation,[41] $\theta_{1}^{2}+\theta_{c}^{2}=\theta_{2}^{2}$ for the case when $\vec{k}_{1}$ enters through the side of the sample (corresponding to $\left.\theta_{1}<0\right)$ and $\vec{k}_{2}$ exits through the surface, or $\theta_{2}^{2}+\theta_{c}^{2}=\theta_{1}^{2}$ when $\vec{k}_{2}$ exits from the side of the sample (corresponding to $\theta_{2}<0$ ) and $\vec{k}_{1}$ enters through the surface. Here, $\theta_{c}$ is taken to be the critical angle for total external reflection of $\mathrm{Si}$, which is $0.102^{\circ}$. Applying these relations, the position of the "T" peaks are given by

$$
\omega_{T}= \pm \frac{\theta_{c}^{2}}{4 \theta}
$$

where positive (negative) $\omega_{T}$ corresponds to $\vec{k}_{2}$ exiting $\left(\vec{k}_{1}\right.$ entering) the side of the sample. It can be shown that the transmitted beam will be observed in an $\omega$ scan as long as $2 \theta<\theta_{c}$. The "T" peaks in Fig. 2.6 are in excellent agreement with these predictions.

For $2 \theta>2 \theta_{c}$ broad Yoneda peaks, $[43,44]$ labeled "Y" in Fig. 2.6, begin to emerge on both sides of the specular position. The Yoneda peaks, which are due to a dynamical enhancement of diffuse scattering arising from surface roughness, are located in the vicinity of

$$
\omega_{Y} \approx \pm\left(\theta-\theta_{c}\right)
$$




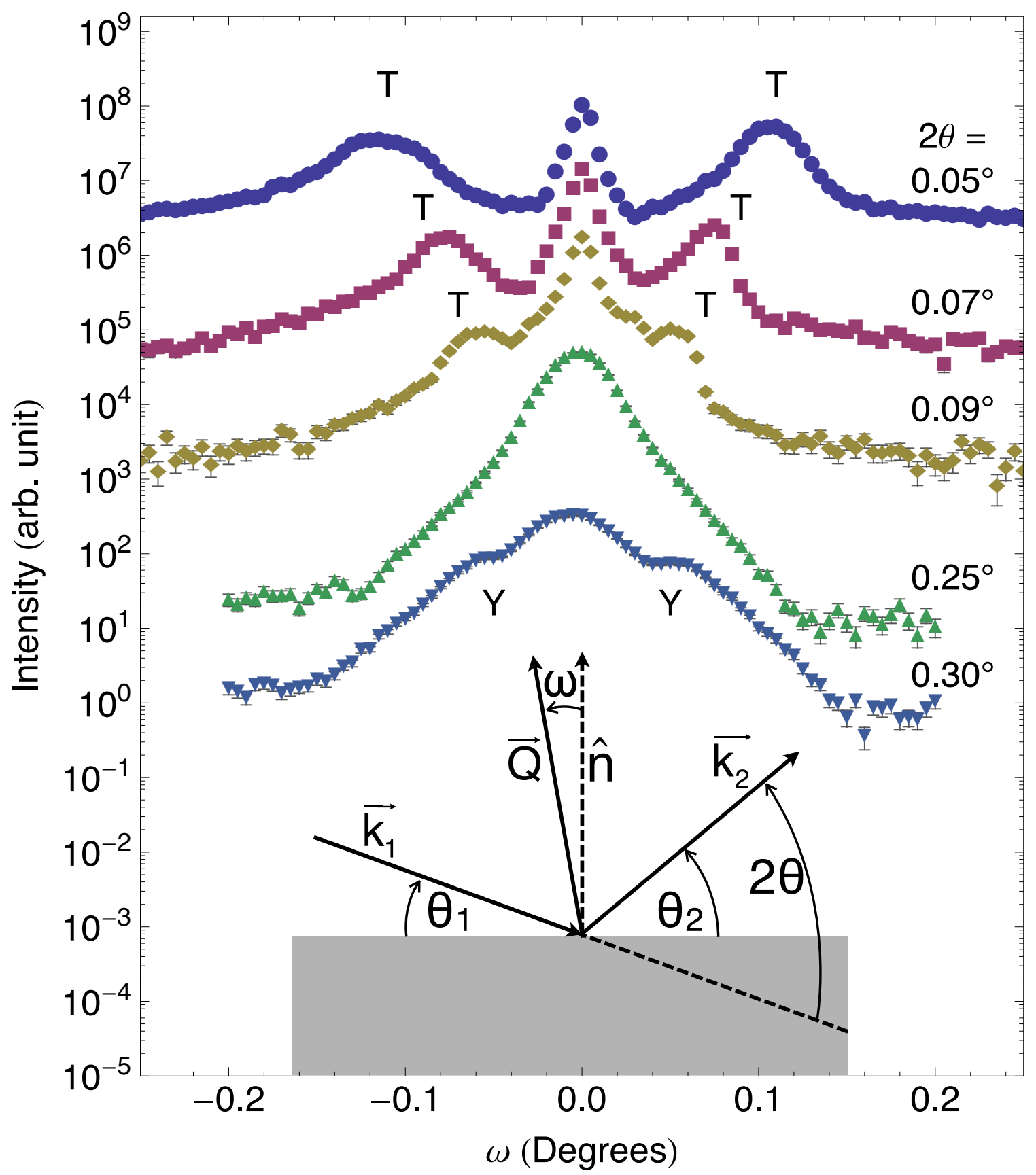

Figure 2.6: A series of transverse line shapes are shown for the 90s sample, where $2 \theta$ is fixed at different angles while rotating the sample angle $\omega$ where $\omega=0$ is the specular reflection condition. The geometry is illustrated in the inset. A pair of transmitted beams, labeled "T", are observed for the lowest three values of $2 \theta$. As discussed in the text, the transmitted peaks are observed for $2 \theta<\theta_{c}^{S i}$ and they move towards $\omega=0$ as $2 \theta$ approaches $\theta_{c}^{S i}=0.10^{\circ}$. Yoneda scattering, labeled "Y", is observed for $2 \theta>2 \theta_{c}$ and they move to higher $|\omega|$ with increasing $2 \theta$. 
The appearance of Yoneda scattering so close to $2 \theta_{c}$ indicates extensive surface roughness on the X-ray length scale and it is accompanied by a precipitous decrease in the specular intensity for $2 \theta>2 \theta_{c}$. In addition to the Yoneda scattering and the transmitted beam, an "anomalous" non-specular scattering feature is also present in the transverse line shapes, which adds to the complexity of extracting the specular component. In between the regions where neither the transmitted beams nor the Yoneda scattering are expected, $\theta_{c}<2 \theta<2 \theta_{c}$, this anomalous feature appears in the form of "shoulders" that broaden the base of the sharp specular peak, as can be seen in Fig. 2.6 for the $2 \theta=0.09^{\circ}$ data. We do not know the origin of this anomalous feature.

The general behavior of the transverse lineshapes shown in Fig. 2.6 is observed for all the samples, although the details depend on the etching time. A useful way to survey the evolution of scattering of the etched samples is through two-dimensional plots of $\omega$ vs. 20, as shown in Fig. 2.7. The plots are false color images of the intensity that were made by meshing the data measured from a series of transverse scans. The scattered intensity is represented linearly and, for visual clarity, the color scale is adjusted to accommodate the intensity range for each sample. Several curves are overlaid to highlight the various scattering features. Reflection occurs in the region between the two dashed horizon lines $(|\omega|<\theta)$ whereas transmission/refraction occurs on curved ridges of intensity indicated by the dot-dashed curves that were calculated from Eq. (2.1) using $\theta_{c}$ of bulk Si. The Yoneda scattering is difficult to see in Fig. 2.7 due to its low intensity; therefore, as a guide, two sets of lines are overlaid. Using Eq. (2.2), the solid thick lines indicate the position of the experimentally observed Yoneda scattering peaks whereas the thin solid lines indicate the position that would be expected for bulk Si. The intersection of the lines at $\omega=0$ gives $2 \theta_{c}$. As can be seen, the experimentally observed $2 \theta_{c}$ decreases with increasing etch time, which points to a progressively decreasing effective electron density at the surface for longer 
etch times.

Another useful way to inspect and appreciate the complexity of the scattering from the etched samples is through three-dimensional plots of intensity vs. $\omega$ and $2 \theta$, as shown in Fig. 2.8. The three-dimensional plots were made by meshing the data measured from a series of transverse scans, which is similar to the method used to make the intensity maps shown in Fig. 2.7. The scattered intensity is represented on a logarithmic scale. Several curves are overlaid to provide visual references. The thin solid curve indicates the $2 \theta_{c}$ position of bulk $\mathrm{Si}$ whereas the thick solid curve indicates the experimentally determined $2 \theta_{c}$ position that was ascertained from the observed Yoneda scattering peaks using Eq. (2.2). In the region where $2 \theta>2 \theta_{c}$, the Yoneda scattering can be seen as pairs of peaks or ridges that emerge on both sides of the specular position. The dot-dashed curve indicates the $\theta_{c}$ position of bulk Si. In the region $2 \theta<\theta_{c}$, the transmitted beam can be seen as pairs of ridges on either side of the specular position that intersect with the specular peak. The "anomalous" non-specular scattering feature, which was described above and observed in Fig. 2.7, can also be identified in Fig. 2.8(a) and (c), in the region between $\theta_{c}$ and $2 \theta_{c}$, as "shoulders" that broaden the base of the sharp specular peak.

We now discuss the method used to determine the specularly reflected intensity. Because the specular reflection should have a transverse width that is resolutionlimited, any additional broadening arises from diffuse scattering or other unwanted sources. In these samples, the diffuse scattering can have a very narrow peak width, on the order of the specular component, which apparently corresponds to correlated disorder that occurs on an unusually long lateral length scale.[44] Therefore, instead of integrating the intensity of the peak at $\omega=0$ in the transverse lineshape irrespective of its width, we extracted the specular component by fitting the peak to two Gaussian curves and a linear sloping background. One Gaussian peak was constrained to have a 


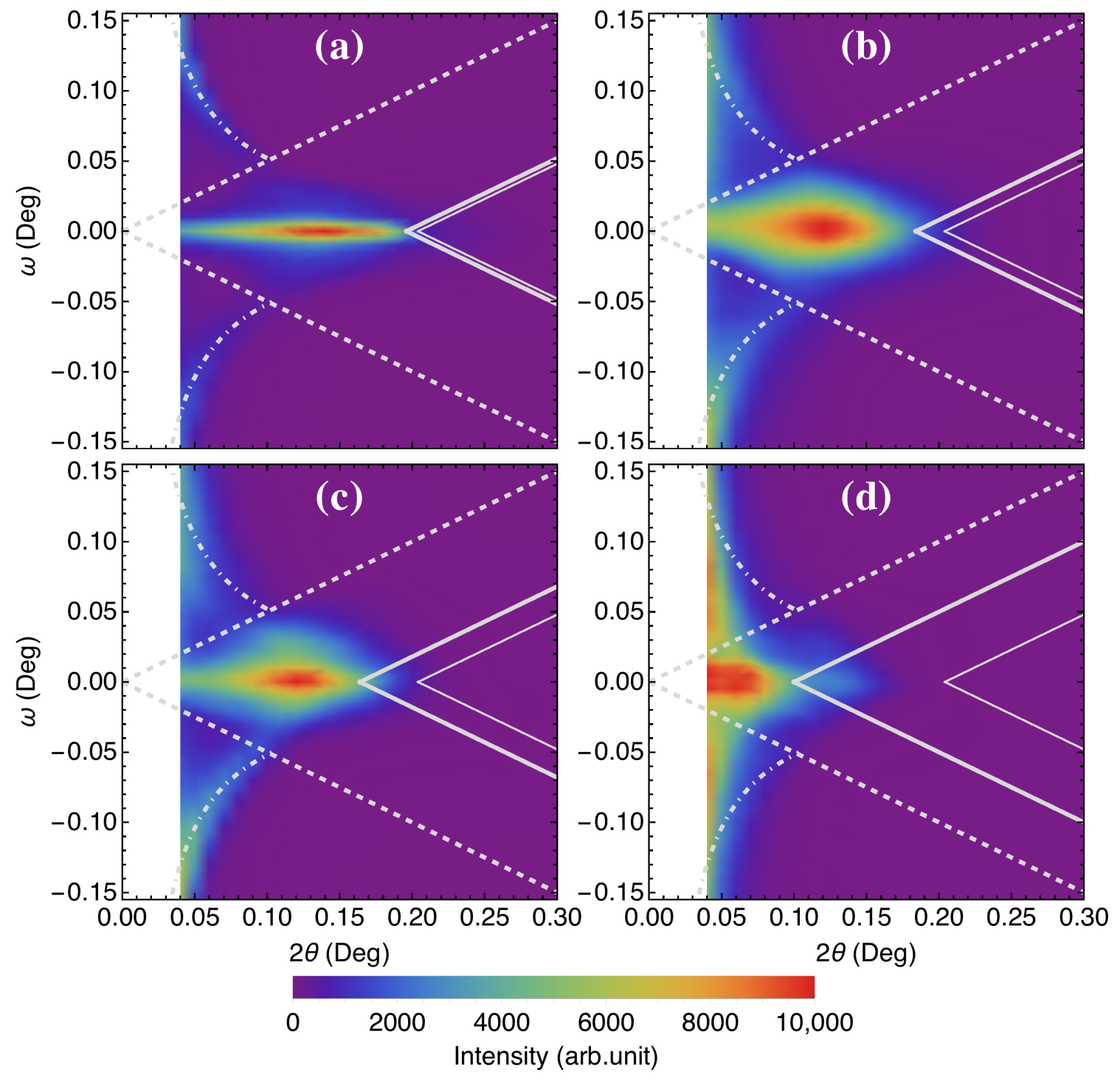

Figure 2.7: Intensity map versus $\omega$ and $2 \theta$ for the (a) $90 \mathrm{~s}$, (b) $5 \mathrm{~m}$, (c) $15 \mathrm{~m}$, and (d) $30 \mathrm{~m}$ samples. The false-color intensity represents a linear scale, which is readjusted for each sample for visual clarity. Several lines and curves are overlaid as a guide and are discussed in the text. 

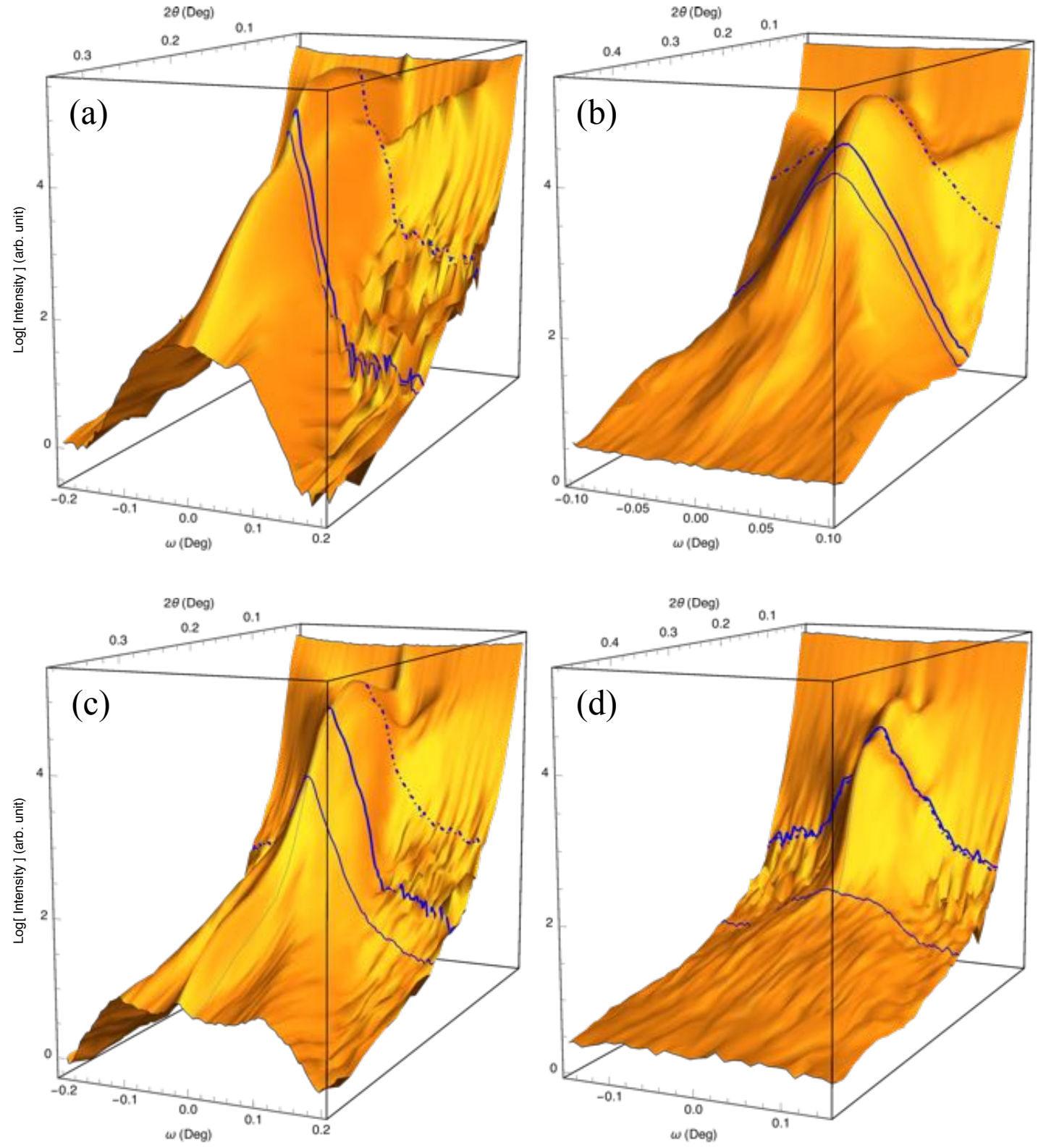

Figure 2.8: Three-dimensional intensity plots versus $\omega$ and $2 \theta$ for the (a) $90 \mathrm{~s}$, (b) $5 \mathrm{~m}$, (c) $15 \mathrm{~m}$, and (d) $30 \mathrm{~m}$ samples. The scattered intensity is represented on a logarithmic scale. Several curves are overlaid as a guide and are discussed in the text. 


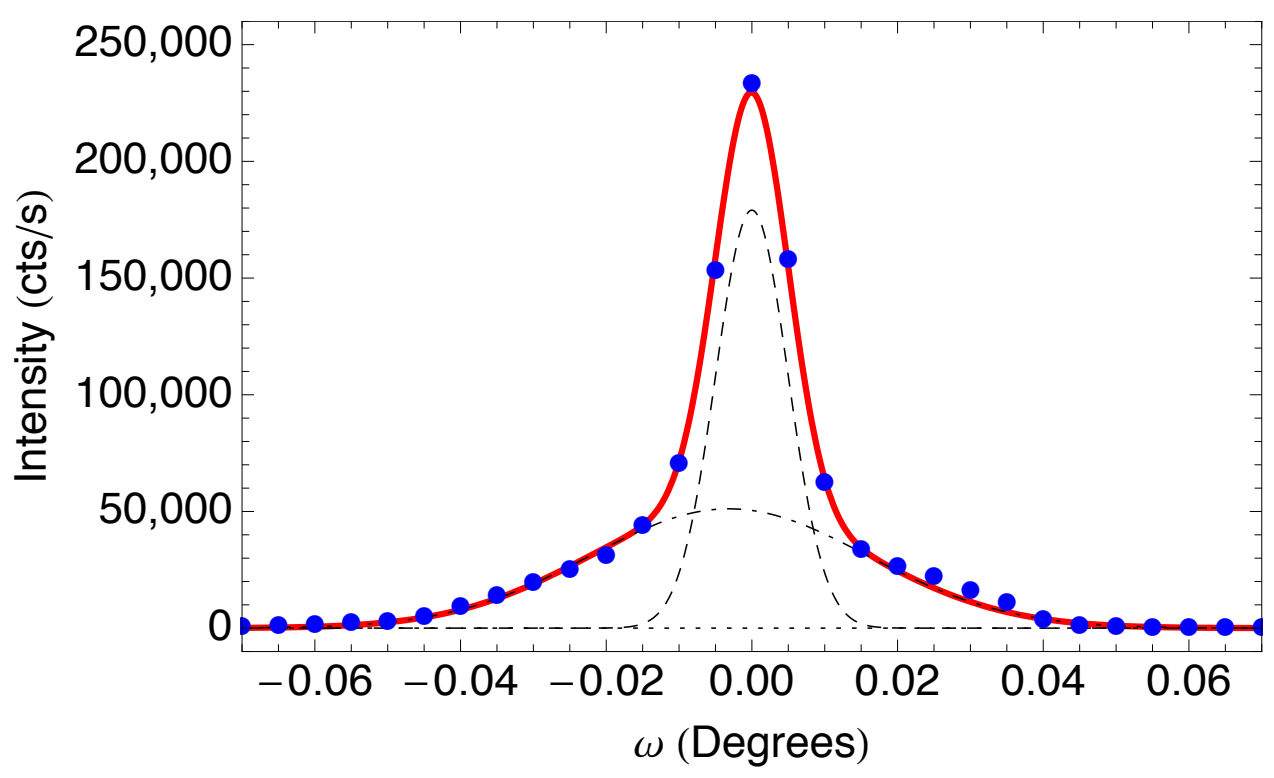

Figure 2.9: Illustration of the method to determine the specular component from a transverse scan measurement (circles) for the 90 s sample at $2 \theta=0.13^{\circ}$. The fitted curve (solid red) is composed of a resolution-limited specular peak having a fixed width (dashed curve), a diffuse peak (dot-dashed curve), and a linear background (dotted line).

pre-determined resolution-limited specular peak width while the other Gaussian was allowed to vary freely in order to accommodate the diffuse scattering in the vicinity of the specular scattering. This procedure, which is illustrated in Fig. 2.9 for the 90s sample at $2 \theta=0.13^{\circ}$, works up until the point where the distinction between the two components becomes ambiguous. The method had a negligible benefit for the 0s sample but it was significant for the other samples where the width increases appreciably above $2 \theta_{c}$.

Because measuring a series of transverse scans as a function of $2 \theta$ with a high point density along $2 \theta$ was not practical with a rotating anode X-ray source, we employed a combination of transverse scan measurements and radial scans along the surfacenormal direction, where the latter can be performed more quickly than the former. Using the transverse peak fitting method described above, the integrated intensities 
of the specular component were extracted from the transverse scan peaks. The radial scans were then used as an interpolation between the $2 \theta$ points obtained from the transverse scans, where the background-subtracted intensities from the radial scans were corrected for the variation in peak-width determined from the transverse scans. In this manner, the measurements could be combined with a sufficiently high point density along $2 \theta$. As a final step, a footprint correction that accounts for the change in X-ray flux when the incident beam spills off the sample at grazing angles, was applied to the measured specular intensity and the resulting intensity was normalized to unity below the critical angle in order to give the specular reflectivity curve.

The resulting X-ray specular reflectivity is plotted versus wavevector transfer, $Q=\frac{4 \pi}{\lambda} \sin \theta$, in Fig. 2.10 for all of the samples where the above analysis was feasible. Samples having etch times of 15 min or greater were too rough to apply this analysis. The XRR data of all samples in Fig. 2.10 clearly exhibit total reflection for $Q$ less than a critical wavevector, $Q_{c} \equiv \frac{4 \pi}{\lambda} \theta_{c}$, although, the $Q_{c}$ is observed to vary significantly among the different samples. Compared to bare $\mathrm{Si}$, it can be seen that the $Q_{c}$ is markedly higher for the 0s sample, indicating that it has a large electron density from $\mathrm{Ag}$ at the surface, although, its $Q_{c}$ is slightly lower than for bulk $\mathrm{Ag}\left(Q_{c}^{A g}=0.062\right.$ $\left.\AA^{-1}\right)$. Remarkably, the $Q_{c}$ for the $90 \mathrm{~s}$ and $5 \mathrm{~m}$ samples is lower than for the bare $\mathrm{Si}$, suggesting that significant density reductions occur at the top interface upon etching. Below, we discuss the density profiles that explain the XRR data.

\subsubsection{Analysis of the X-ray Specular Reflectivity}

The X-ray specular reflectivity data were modeled using the REFLPAK software [45] that is based on the Parratt method.[46] The structural model consists of one or more layers above a semi-infinite $\mathrm{Si}$ substrate, as shown schematically in the inset to 


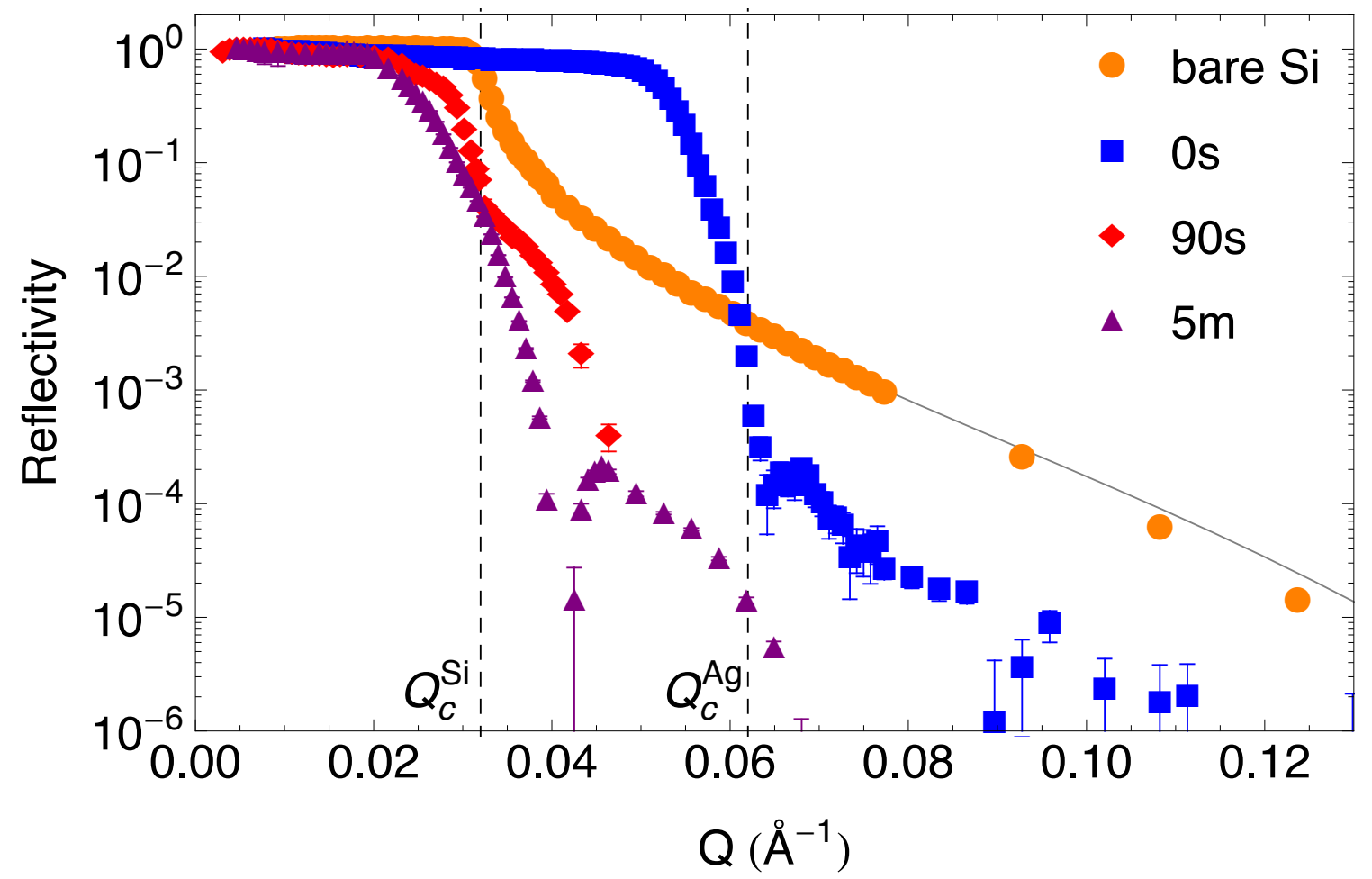

Figure 2.10: X-ray specular reflectivity data for the bare $\mathrm{Si}, 0 \mathrm{~s}, 90 \mathrm{~s}$, and $5 \mathrm{~m}$ samples. The solid curve is a fit to the bare $\mathrm{Si}$ data, which includes $22 \AA$ of $\mathrm{SiO}_{2}$ on the surface. For reference, vertical dashed lines show the expected location of total reflection for bulk $\mathrm{Ag}$ and $\mathrm{Si}, Q_{c}^{A g}=0.062 \AA^{-1}$ and $Q_{c}^{S i}=0.032 \AA^{-1}$, respectively. 
Fig. 2.11. Each layer, numbered as $j$ with $j \geq 1$, has four parameters: layer thickness, $D_{j}$, interface width, $\sigma_{j}$, and two optical constants, which are the critical wavevector squared, $Q_{c j}^{2}$, and the absorption, $\mu_{j}$. The interface between two layers is calculated as an error function [45] that connects the optical constants of the two adjoining layers, $j$ and $j+1$, so that $\sigma_{j}$ is the width of this error function. In order to obtain the elemental composition in the sample, the optical constants of the individual layers can be related to linear combinations of the elemental constituents according to:

$$
\begin{aligned}
\mu_{j} & =c_{j}^{A g} \mu^{A g}+c_{j}^{S i} \mu^{S i} \\
Q_{c j}^{2} & =c_{j}^{A g} Q_{c}^{2 A g}+c_{j}^{S i} Q_{c}^{2 S i} \\
1 & =c_{j}^{A g}+c_{j}^{S i}+c_{j}^{v}
\end{aligned}
$$

where $c_{j}^{A g}$ and $c_{j}^{S i}$ are the elemental compositions of Ag or Si in layer $j$ and $c_{j}^{v}$ allows for densities lower than the bulk elements $\left(0 \leq c_{j} \leq 1\right)$. The elemental optical constants [42] are calculated from $Q_{c}^{2}=16 \pi r_{c} \rho^{N} f_{1}$ and $\mu=2 \lambda r_{c} \rho^{N}\left|f_{2}\right|$, where $r_{c}$ is the classical electron radius, $\rho^{N}$ is the number density of the atomic species, and $f_{1}$ and $f_{2}$ are the real and imaginary parts of the atomic form factor, respectively. The elemental optical constants are given as $Q_{c}^{2 S i}=9.97 \times 10^{-4} \AA^{-2} ; \mu^{S i}=2.00 \times 10^{-7} \AA^{-1}$ and $Q_{c}^{2 A g}=3.82 \times 10^{-3} \AA^{-2} ; \mu^{A g}=3.28 \times 10^{-6} \AA^{-1}$. In the analysis, $D_{j}, \sigma_{j}, Q_{c j}^{2}$, and $\mu_{j}$ of the layers above the Si substrate were allowed to refine and, for each sample, the fewest number of layers that could explain the data were used in the model. The Si composition of an upper layer was constrained to not exceed the composition of the layer below, $c_{j+1}^{S i} \leq c_{j}^{S i}$, corresponding to the lower Si density expected in a layer containing the NWs. Constraining $Q_{c j}^{2}$ and $\mu_{j}$ according to Eq. (2.3) was essential for determining the Ag composition profiles and for distinguishing between structural models. This procedure was enabled by the photoelectric absorption of Ag being 


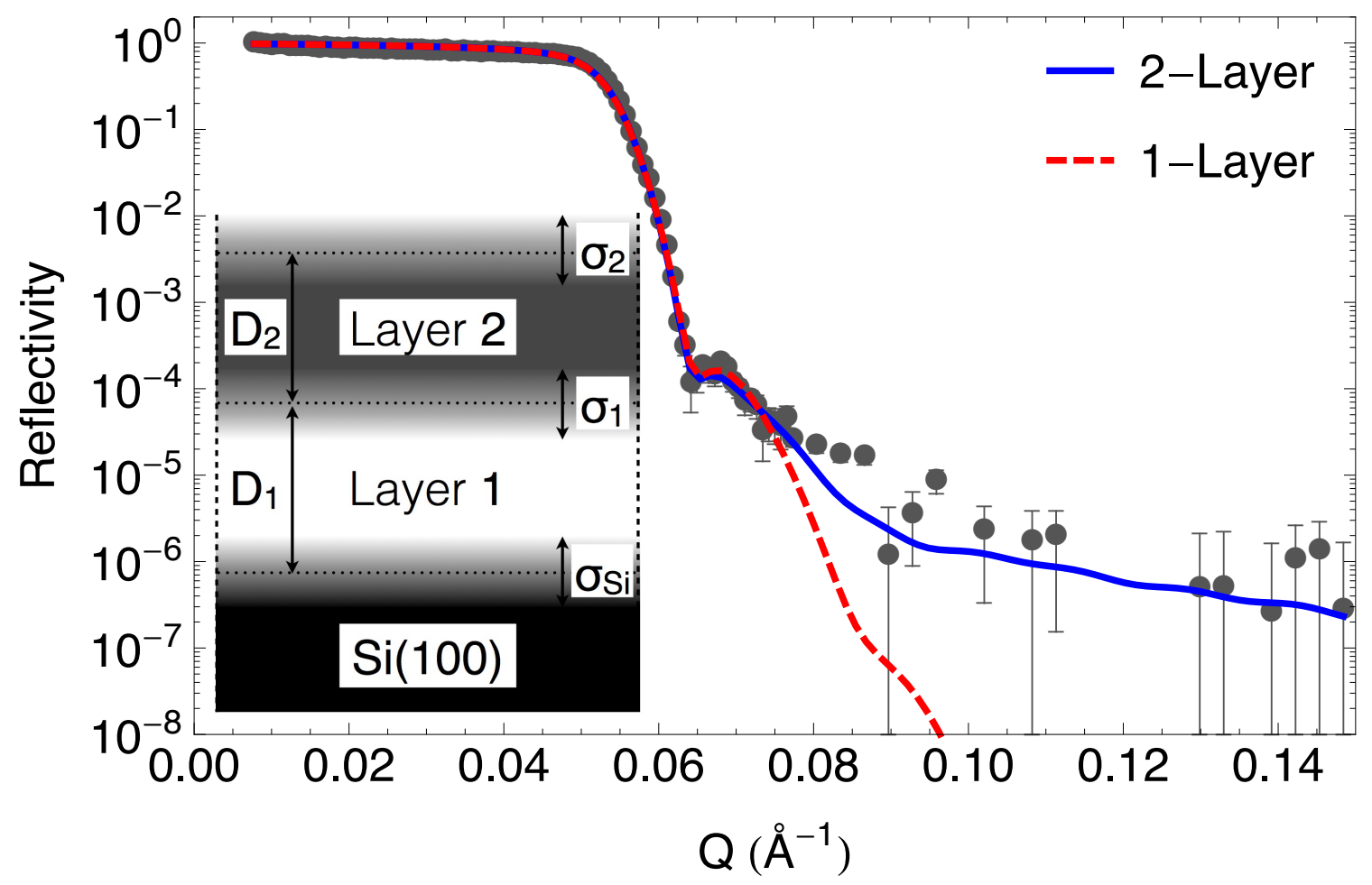

Figure 2.11: X-ray specular reflectivity data for the 0s sample is shown from measurements of both radial scans and integrated specular intensity obtained from transverse scans. Fitted curves to the data are shown for the 1-layer and 2-layer models. The inset shows a schematic side view of the 2-layer model used to calculate the specular reflectivity.

much stronger than for Si.

Figure 2.11 shows the specular reflectivity data for the 0s sample along with fitted curves for a 1-layer and a 2-layer model. In the region below $Q=0.075 \AA^{-1}$, both model curves are similar and explain the data well. Above $Q=0.075 \AA^{-1}$, however, the reflectivity of the 1-layer model decays more rapidly with $Q$ than the data, which suggests the presence of a sharp interface in the sample. Because a 1-layer model cannot provide the needed broad interface at the surface simultaneously with a sharp buried interface, a 2-layer model was used to introduce a small but abrupt density step at the $\mathrm{Ag} / \mathrm{Si}$ interface that explains the larger reflectivity observed at higher $Q$.

The corresponding $Q_{c}^{2}$ profiles for the 1-layer and 2-layer models are shown in 
Fig. 2.12(a). The peak in $Q_{c}^{2}$ is dominated by the Ag contribution, which is located on top of the Si substrate. The two models produce very similar profiles except at the $\mathrm{Ag} / \mathrm{Si}$ interface where the 2-layer model enables a very small 'step' in density compared to the smooth transition of the 1-layer model. As can be seen, the small step has a minor effect on the $Q_{c}^{2}$ profile but it has a significant impact on the reflectivity curve at high $Q$, which illustrates the high sensitivity of XRR to the interface widths.

The reflectivity was found to be less sensitive to the amount of Ag adjacent to the Si substrate, layer 1, than at the top surface, layer 2. This is because $\mu_{1}$ is much smaller than $\mu_{2}$. As a result, a range of composition profiles at the $\mathrm{Ag} / \mathrm{Si}$ interface can be determined from the 2-layer model according to Eq. (2.3) and the limiting range of profiles are shown in Fig. 2.12(b) and (c). The sharper Ag/Si interface of Fig. 2.12(b) could correspond to Ag nanoparticles resting on top of a still flat substrate, whereas the broader profile of Fig. 2.12(c) corresponds to the nanoparticles embedded into the Si by $\sim 100 \AA$. Interestingly, the peak value of both $c^{A g}$ profiles, $\sim 73 \%$, is similar to the value expected for the close-packed density of spherical particles, which is $74 \%$. The FWHM of the $c^{A g}$ peak in Fig. 2.12(b) and (c), 300 and $260 \AA$, respectively, are also similar to the initial grain size determined from XRD in Fig. 2.5 and the average lateral diameter determined from SEM in Fig. 2.2(c) and (d).

A convenient method to quantify the amount of $\mathrm{Ag}$ in the sample is to define a total effective Ag thickness, $t^{A g}$, which is the thickness of a Ag layer that would occur if all of the $\mathrm{Ag}$ in the sample was combined into a single layer having a composition of $100 \%$. It is determined by integrating $c^{A g}$ over the sample thickness, $t^{A g}=\int c^{A g} d z$. The $t^{A g}$ in Fig. 2.12(b) and (c) are $233 \AA$ and $193 \AA$, respectively. These values are remarkably consistent with the $t^{A g}$ of close-packed Ag nanoparticles with the initial grain size $(74 \%$ of $300 \AA)$ determined by XRD shown in Fig. 2.5. It also suggests that each $\mathrm{Ag}$ nanoparticle is a single crystalline grain. Given that reflectivity is 


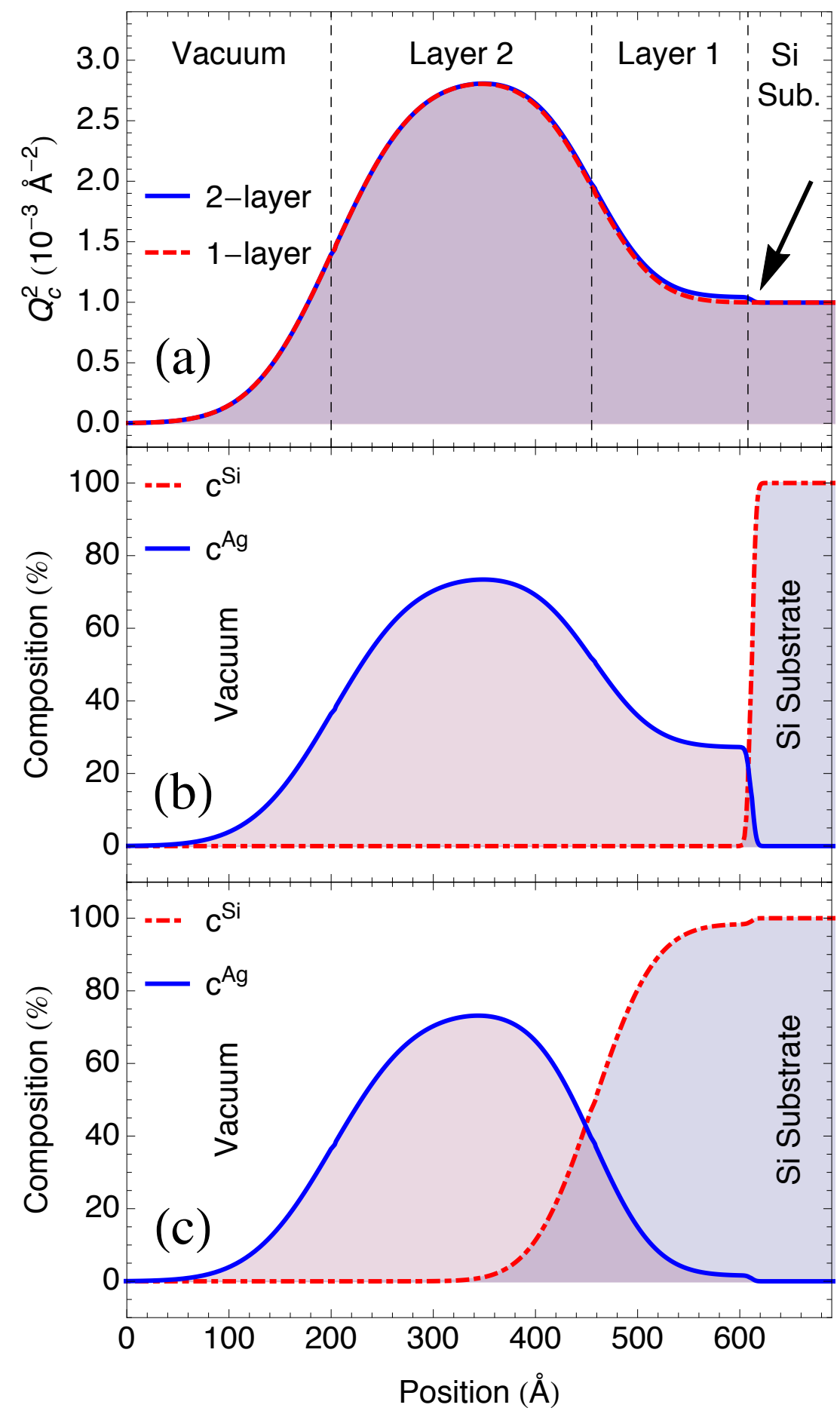

Figure 2.12: (a) $Q_{c}^{2}$ profiles for the 0s sample that were determined from the 1-layer and 2-layer models used to fit the data in Fig. 2.11. The arrow points to the density 'step' discussed in the text. (b) and (c) show the limiting range of composition profiles that can be determined from the data. 
insensitive to the crystallinity of the material and that its analysis is completely independent of the diffraction results, the consistency between these very different X-ray measurements gives confidence in the method used to extract the specular scattering from the transverse scans. A summary of best-fit parameter values, their uncertainties, as well as $t^{A g}$ are given in Table 2.1 for all of the samples studied by XRR.

The specular reflectivity data for the 90s sample along with the best fitted curve to a 2-layer model is shown in Fig. 2.13. The value of $D_{1}, \sim 200 \AA$, being much smaller than $D_{2}$, was determined by the shape of the reflectivity above $Q \approx 0.032 \AA^{-1}$. It was found that $D_{2}$ could be varied over a broad range of values without appreciably changing the reflectivity curve, although, other parameters would change in order to compensate for $D_{2}$. Therefore, the parameters were refined by using fixed values of $D_{2}$ and a range of acceptable $D_{2}$ values, $1500 \leq D_{2} \leq 4000 \AA$, was determined by requiring physically reasonable values of composition, defined by Eq. (2.3) $\left(c_{j}\right.$ bounded between 0 and 1 at every position). The values of $Q_{c 2}^{2}$ and $Q_{c 1}^{2}$ were very sensitive to the shape of the reflectivity near the total reflection, $Q \approx 0.03 \AA^{-1}$, and the 'bump' around $Q=0.035 \AA^{-1}$, respectively.

Figure 2.14(a) shows the corresponding $Q_{c}^{2}$ profiles at the limiting values of $D_{2}$. A $\sim 200 \AA$ wide peak is observed in both cases and the height of the peak is greater than $Q_{c}^{2 S i}$, which suggests the presence of $\mathrm{Ag}$ in layer 1 and at the etch front. Fig. 2.14(b) and (c) show the corresponding range of composition profiles and they confirm the presence of a $\sim 200 \AA$ thick $\mathrm{Ag}$ feature adjacent to the Si substrate, independent of the value of $D_{2}$. To within experimental sensitivity, this $\sim 200 \AA$ step in Ag density is similar to the Ag nanoparticle size and, therefore, suggests Ag nanoparticles residing at the etch front, which is remarkably abrupt compared to the length-scale of $D_{2}$. Additionally, however, a major conclusion of our study is that a large amount of $\mathrm{Ag}$ 


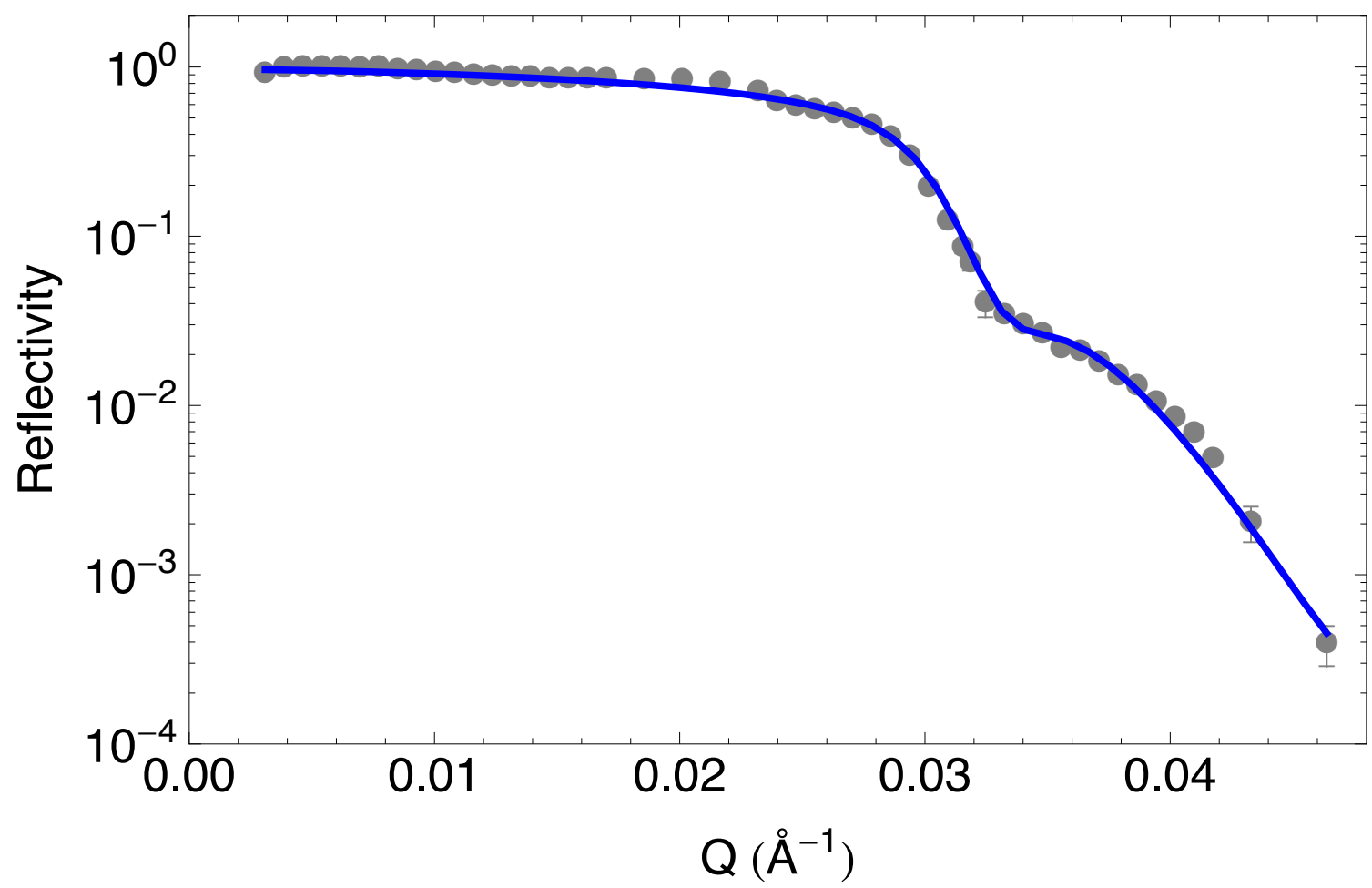

Figure 2.13: X-ray specular reflectivity data for the 90s sample (circles) are shown with the best-fit curve of a 2-layer model (solid curve). 
is distributed along the NWs, as can be seen from Fig. 2.14(b) and (c).

The specular reflectivity data for the $5 \mathrm{~m}$ sample is shown in Fig. 2.15 along with the fitted curves for a 2-layer and a 3-layer model. Although the 2-layer model generally reproduces the overall shape of the reflectivity data, it is unable to capture the pronounced rounding of the reflectivity curve in the total reflection region for $Q<0.03 \AA^{-1}$. The rounding is an indication of a large extended interface that would effectively broaden the critical angle region. As can be seen in Fig. 2.15, using an additional layer captures the shape of the reflectivity in the critical angle region and below. The thicknesses of the top two layers in the 3-layer model, $D_{2}$ and $D_{3}$, could be changed over a broad range without appreciably changing the reflectivity curve. Therefore, the parameter relationships were explored by separately stepping through fixed values of $D_{2}$ and $D_{3}$ while allowing the other parameters to refine.

$D_{2}$ and $D_{3}$ were found to be roughly equivalent and the range of the combined thickness of the top two layers, $D_{2}+D_{3}$, that produced an acceptable fit while providing physically reasonable parameter values in accordance with Eq. (2.3), was between 4000-7000 $\AA$. The $Q_{c}^{2}$ of the three layers were particularly sensitive to the shape of the reflectivity for the total reflection region, $Q<0.02 \AA^{-1}$, the change in curvature near $Q=0.035 \AA^{-1}$, and the broad oscillation after the cusp, $Q>0.04 \AA^{-1}$. However, neither model was able to accommodate the additional reflectivity between $Q=0.044$ and $0.05 \AA^{-1}$.

The corresponding $Q_{c}^{2}$ profiles are shown in Fig. 2.16(a). The two 3-layer model curves represent the range limits of the acceptable combined thickness of $D_{2}+D_{3}$. Compared to the 2-layer model, it can be seen that the addition of the third layer gives a slight additional step in the density profile that is responsible for the rounding of the reflectivity data in Fig. 2.15 below the critical angle near $Q=0.02 \AA^{-1}$. Near the etch front, the height of the $Q_{c}^{2}$ profile is greater than $Q_{c}^{2 S i}$, which indicates that 


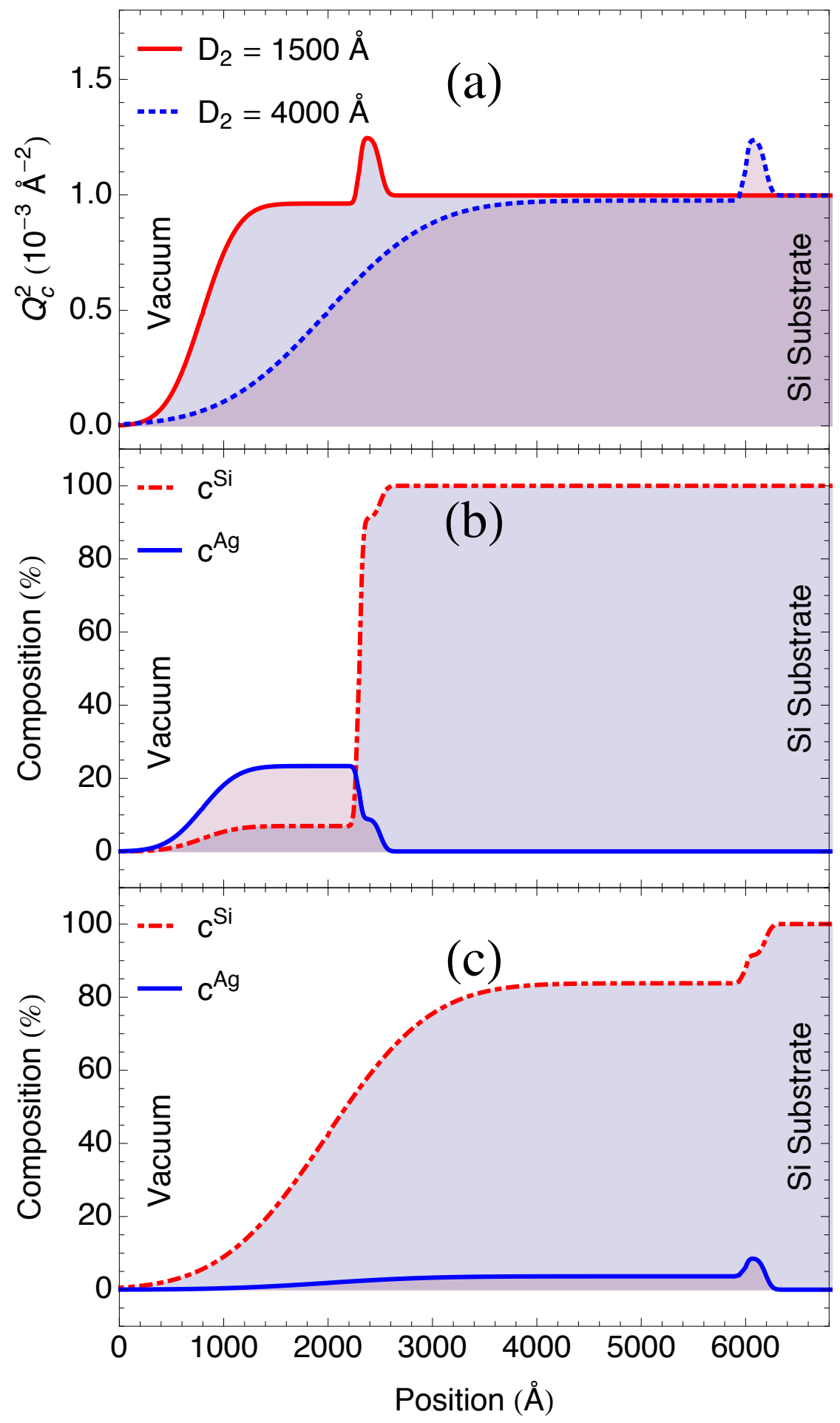

Figure 2.14: (a) $Q_{c}^{2}$ profiles for the 90 s sample at the limits of $D_{2}$ that produced an acceptable fit to the XRR data in Fig. 2.13. (b) and (c) are material composition profiles determined from the $Q_{c}^{2}$ profiles in (a), for $D_{2}=1500$ and $4000 \AA$, respectively. 


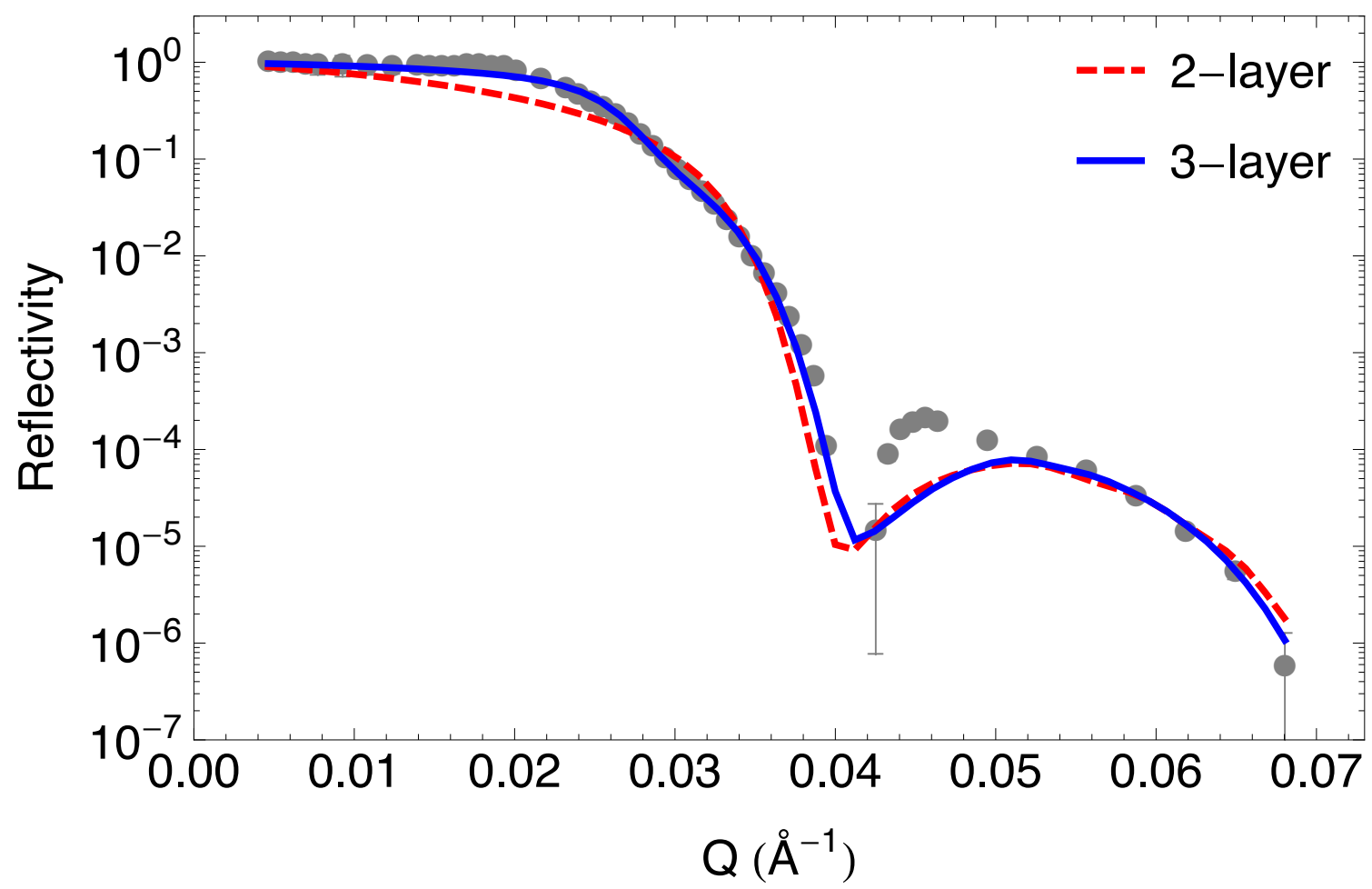

Figure 2.15: X-ray specular reflectivity data (circles) for the $5 \mathrm{~m}$ sample along with fitted curves for the 2-layer and 3-layer models. 
Ag is broadly distributed along the NWs. Independent of whether a 2-layer or 3-layer model is used, a $\sim 160 \AA$ thick feature appears adjacent to the Si substrate, in layer 1, similar to the 90 s sample. This feature results from the broad oscillation in the reflectivity above $Q=0.04 \AA^{-1}$. Figure 2.16(b) shows the composition profile for the midrange of acceptable fitted curves to the 3-layer model. Notably, the profile shows that the Ag is distributed throughout the etched layers with a composition gradient

that increases toward an abrupt etching front terminated with a $\sim 160 \AA$ thick $\mathrm{Ag}$ feature, which is likely limited by the shape of the Ag nanoparticles at the interface.

Finally, we can use the Yoneda scattering, which is diffuse rather than specular scattering, to independently corroborate the general observation that the specular reflectivity exhibits a decreasing effective $Q_{c}^{2}$ with increasing etch time. The $Q_{c}^{2}$ was extracted from the position of the Yoneda scattering peaks in Fig. 2.7 according to Eq. (2.2) and it is plotted in Fig. 2.17 along with the $Q_{c}^{2}$ values of the top layer obtained from modeling the specular reflectivity. As can be seen, Fig. 2.17 shows excellent agreement between the Yoneda scattering and the specular reflectivity. After an initial precipitous drop, the $Q_{c}^{2}$ of the etched surface decreases linearly with etch time at a rate of $\sim 2.5 \times 10^{-5} \AA^{-2} \mathrm{~min}^{-1}$. This clear trend indicates a reduction in electron density near the surface with etch time, which is due to the progressive diffusion of $\mathrm{Ag}$ along the SiNWs during the etch. It is noteworthy that both the specular and diffuse scattering confirm this point.

\subsection{Discussion}

Our results provide important new insight into the amount and depth distribution of Ag during the formation of SiNWs by MACE and this new information is essential if one is to understand the mechanism by which SiNWs are formed. Our study also 


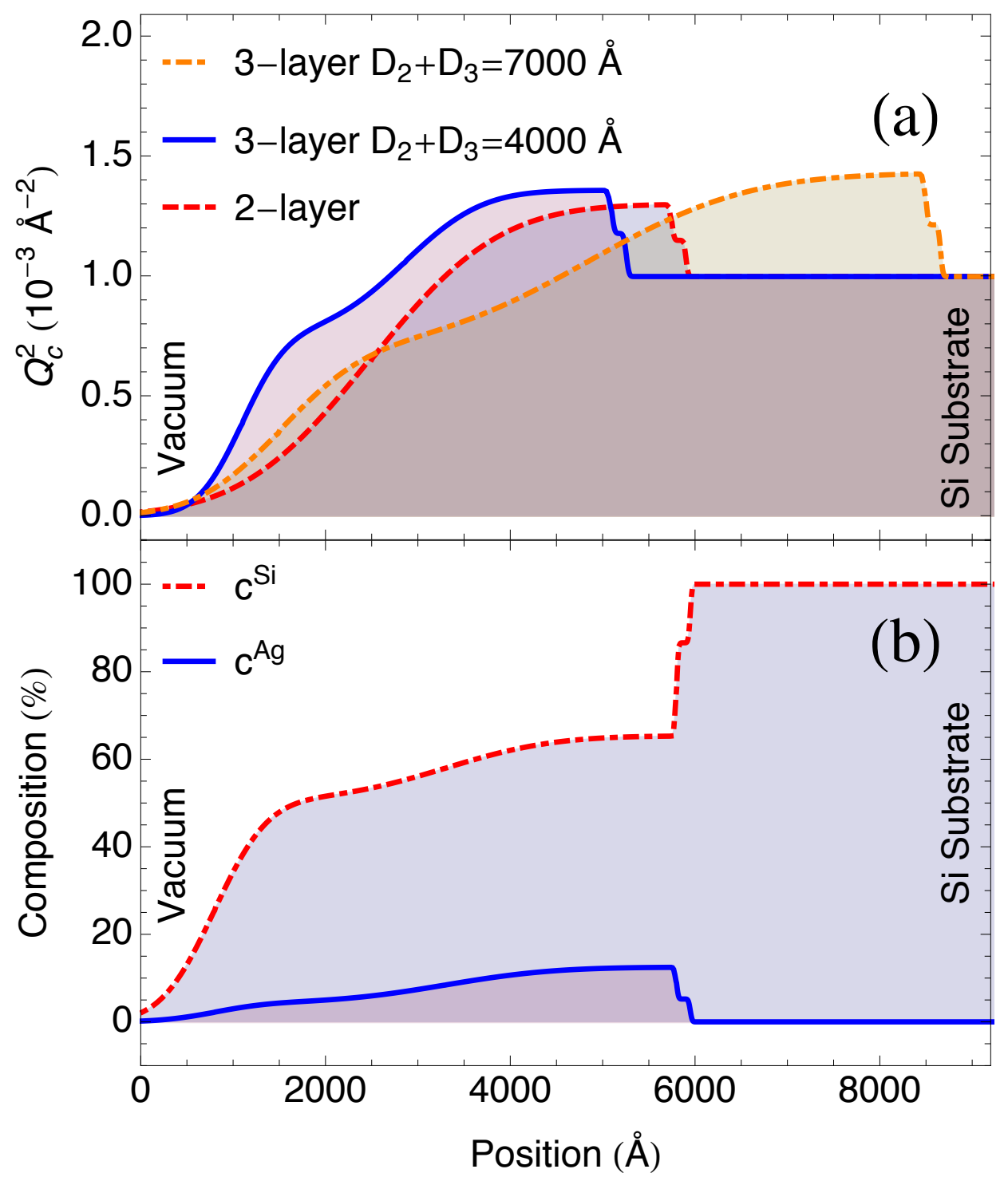

Figure 2.16: (a) $Q_{c}^{2}$ profiles for the $5 \mathrm{~m}$ sample using a 2-layer model and 3-layer model. The 3-layer model profiles are at the limits of $D_{2}+D_{3}$ that produced an acceptable fit to the XRR data shown in Fig. 2.15. (b) composition profile for the midrange of acceptable fits using the 3-layer model. 


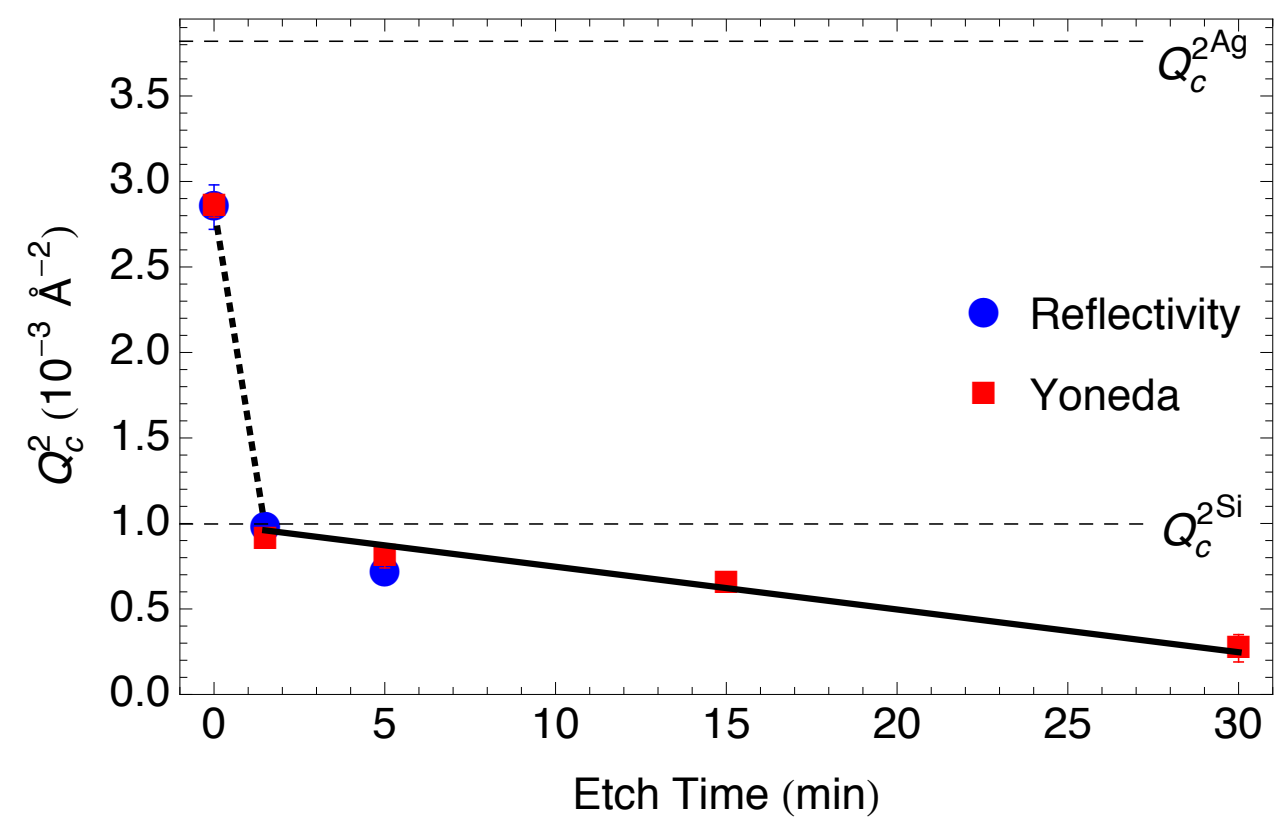

Figure 2.17: The $Q_{c}^{2}$ values determined from the Yoneda scattering as a function of etch time are compared to those obtained for the top layer from specular reflectivity. The solid line is a fit to the Yoneda scattering $Q_{c}^{2}$ values and, for reference, the horizontal dashed lines indicate the values for bulk $\mathrm{Si}$ and $\mathrm{Ag}$.

Table 2.1: Summary of the best-fit parameter values determined from X-ray specular reflectivity and the corresponding effective Ag thickness. Error bars were obtained by determining the range that the parameter could be varied while maintaining an acceptable fit to the data.

\begin{tabular}{lcccccc} 
Sample & Layer, $j$ & $Q_{c j}^{2}\left(10^{-3} \AA^{-2}\right)$ & $\mu_{j}\left(10^{-6} \AA^{-1}\right)$ & $D_{j}(\AA)$ & $\sigma_{j}(\AA)$ & $t^{A g}(\AA)$ \\
\hline \hline \multirow{2}{*}{$0 \mathrm{~s}$} & 2 & $2.85 \pm 0.1$ & $2.5 \pm 0.2$ & $250 \pm 40$ & $140 \pm 50$ & \\
& 1 & $1.07 \pm 0.03$ & $0.6 \pm 0.3$ & $155 \pm 15$ & $120 \pm 20$ & $300 \pm 100$ \\
& $\mathrm{Si}$ & 0.997 & 0.20 & $\infty$ & $10 \pm 10$ & \\
\hline \multirow{3}{*}{$90 \mathrm{~s}$} & 2 & $0.97 \pm 0.01$ & $0.6 \pm 0.2$ & $2750 \pm 1250$ & $1200 \pm 600$ & \\
& 1 & $1.26 \pm 0.02$ & $0.8 \pm 0.2$ & $199 \pm 7$ & $40 \pm 20$ & $290 \pm 120$ \\
& $\mathrm{Si}$ & 0.997 & 0.20 & $\infty$ & $130 \pm 60$ & \\
$5 \mathrm{~m}$ & 3 & $0.71 \pm 0.05$ & $0.6 \pm 0.2$ & $2750 \pm 750$ & $1400 \pm 400$ & \\
& 2 & $1.39 \pm 0.07$ & $1.2 \pm 0.2$ & $2750 \pm 750$ & $2300 \pm 600$ & $330 \pm 140$ \\
& 1 & $1.19 \pm 0.03$ & $0.9 \pm 0.5$ & $161 \pm 7$ & $60 \pm 10$ & \\
& $\mathrm{Si}$ & 0.997 & 0.20 & $\infty$ & $49 \pm 6$ & \\
\hline
\end{tabular}


demonstrates that, under appropriate conditions and with special care in identifying the specular component of reflection, useful information on highly rough interfaces can be obtained from X-ray specular reflectivity methods. The etched Si interfaces studied here are considerably rougher than what is usually studied with this technique. The large contrast between the Ag and Si optical constants as well as the high photoelectric absorption of Ag were essential to our ability to determine the elemental composition profiles.

An important and surprising result is that the total amount of Ag in the sample does not change with the etch time. This fact was established independently by two quite different X-ray measurements: the X-ray diffraction intensity, which measures the amount of crystalline Ag, and X-ray specular reflectivity, which measures the total amount of $\mathrm{Ag}$ in terms of its spatial distribution, independent of the crystal structure. As shown in Fig. 2.5(a), the $\mathrm{Ag}(111)$ integrated Bragg intensity is constant across the samples, indicating that the amount of crystalline Ag does not change with the etch time. Similarly, the effective Ag thickness, $t^{A g}$, shown in Fig. 2.18, which was obtained from our X-ray specular reflectivity analysis, also does not change with etch time. Moreover, the average $t^{A g}$ from Fig. 2.18, $\sim 300 \AA$, is remarkably close to the initial grain size determined by X-ray diffraction, shown in Fig. 2.5(b), and it is also similar to the average lateral width of the Ag nanoparticles seen in the SEM images of Fig. 2.2(c) and (d). Therefore, it appears that the initial crystalline Ag grain size is determined by the initial layer thickness of $\mathrm{Ag}$ that is deposited before etching occurs. The fact that the initial amount of Ag does not change during etching indicates that the Ag within the NW region does not exit the sample into the etching bath. It is an important constraint for developing an understanding of how the Ag nanoparticles lead to the formation of the SiNWs.

The crystalline Ag grain size, as seen in the XRD results of Fig. 2.5(b), was 


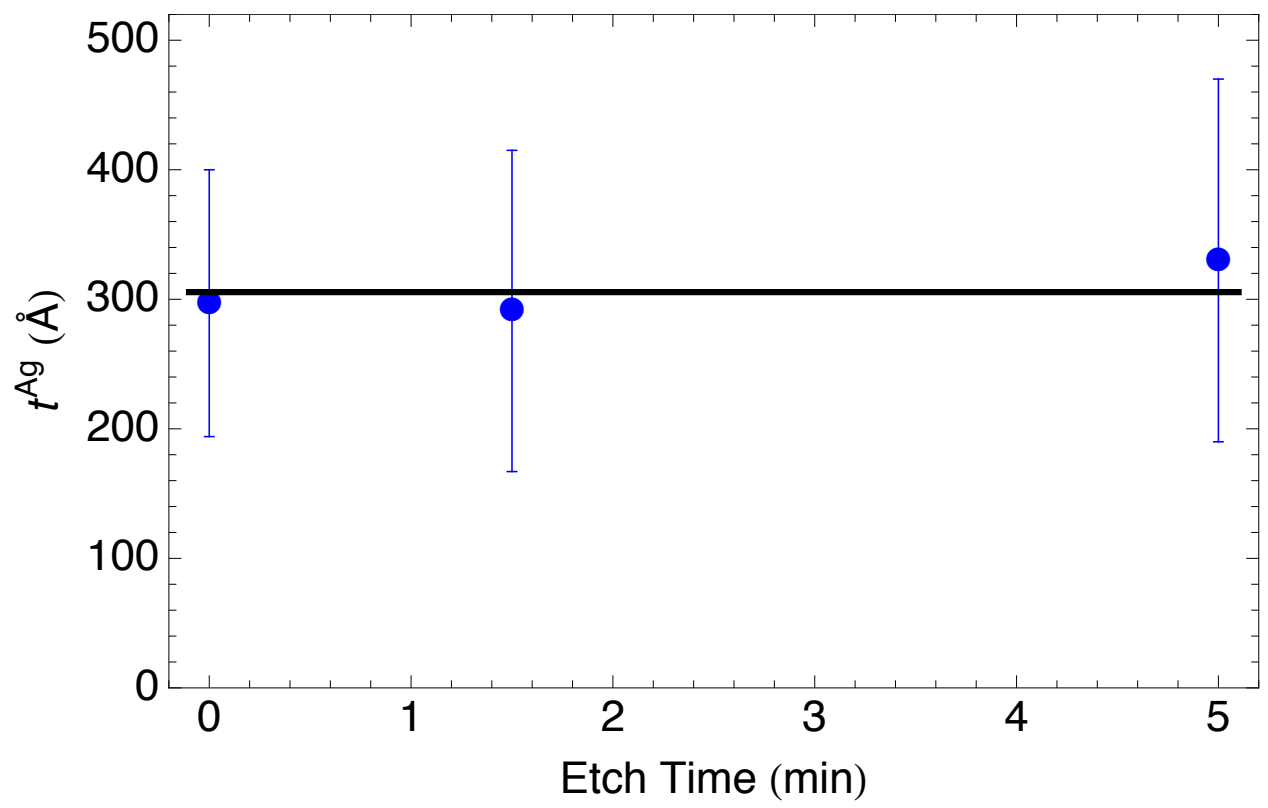

Figure 2.18: Effective Ag thickness, $t^{A g}$, determined from XRR. The line indicates the average $t^{A g}$ of $307 \AA$.

observed to increase with etch time, nearly doubling during $30 \mathrm{~min}$ of etching. This coarsening indicates that there is a significant amount of mass transport of Ag during the etch process. Given that the total amount of $\mathrm{Ag}$ does not change during the etching, however, the coarsening of the Ag nanoparticles suggests that the Ag mobility is short-ranged and confined to be within the NW region. The coarsening of the $\mathrm{Ag}$ crystalline grain size also does not alter the diameter of the SiNWs. Even though the Ag grains nearly double in size, the SiNW diameter is uniform over its length, as seen in the SEM images of Fig. 2.3(g); no tapering is observed over the length of the wires. Other studies have also reported uniform diameter wires.[11, 40] Therefore, it is evident that the SiNW diameter is independent of the Ag coarsening.

The most striking result of our investigation relates to the broad distribution of $\mathrm{Ag}$ nanoparticles along the NWs. Earlier studies have suggested that Ag is concentrated either at the surface[30-32] or at the etch front[33, 40] of the SiNWs. In contrast, our XRR measurements definitively demonstrate that the $\mathrm{Ag}$ composition is distributed 
along the SiNWs. This result is independently confirmed by our EDS analysis that detects Ag on the NW walls, although, one should note that determining the Ag distribution by electron microscopy is both qualitative and challenging due to shadowing effects by the SiNWs themselves, as well as due to artifacts arising from the unavoidable cleaving of the sample that is necessary to obtain a cross-sectional view. Models with Ag concentrated only at the top or bottom of the SiNWs completely fail to fit our X-ray reflectivity data for the etched samples. The reason X-ray reflectivity is so clear on this point is because of its relation to a Fourier transform: a large amount of Ag localized near an interface would lead to a significantly higher reflectivity at large $Q$, which is not observed (see Fig. 2.10). Indeed, the reflectivity decreases precipitously close to the total reflection. Furthermore, if the Ag was concentrated at an interface, the $Q$ for total reflection would remain near the value for Ag rather than decrease below the Si value, which is clearly demonstrated in Fig. 2.17.

For all etched samples, we find a Ag concentration gradient that increases toward the etch front at the Si interface. With this concentration gradient, Fick's law would predict that Ag should diffuse away from the Si interface, with some Ag exiting into the etching bath. Because we have shown that Ag does not leave the sample upon etching, it is apparent that there must be an opposing chemical force, which is greater in magnitude than that for diffusion, in order to attract the Ag nanoparticles towards the etch front at the Si interface while leaving a trail of Ag nanoparticles deposited along the SiNWs.

Our analysis has also revealed that a $\sim 170 \AA$ feature is present at the etch front for different etch times, as shown for example in the ${Q_{c}}^{2}$ profiles of Fig. 2.14 and 2.16. The feature corresponds to an abrupt etching front having a width on the order of the Ag nanoparticle radius. It is notable that this aspect of the etching interface does not significantly broadened over time, up to at least $5 \mathrm{~min}$, suggesting a uniform etch 


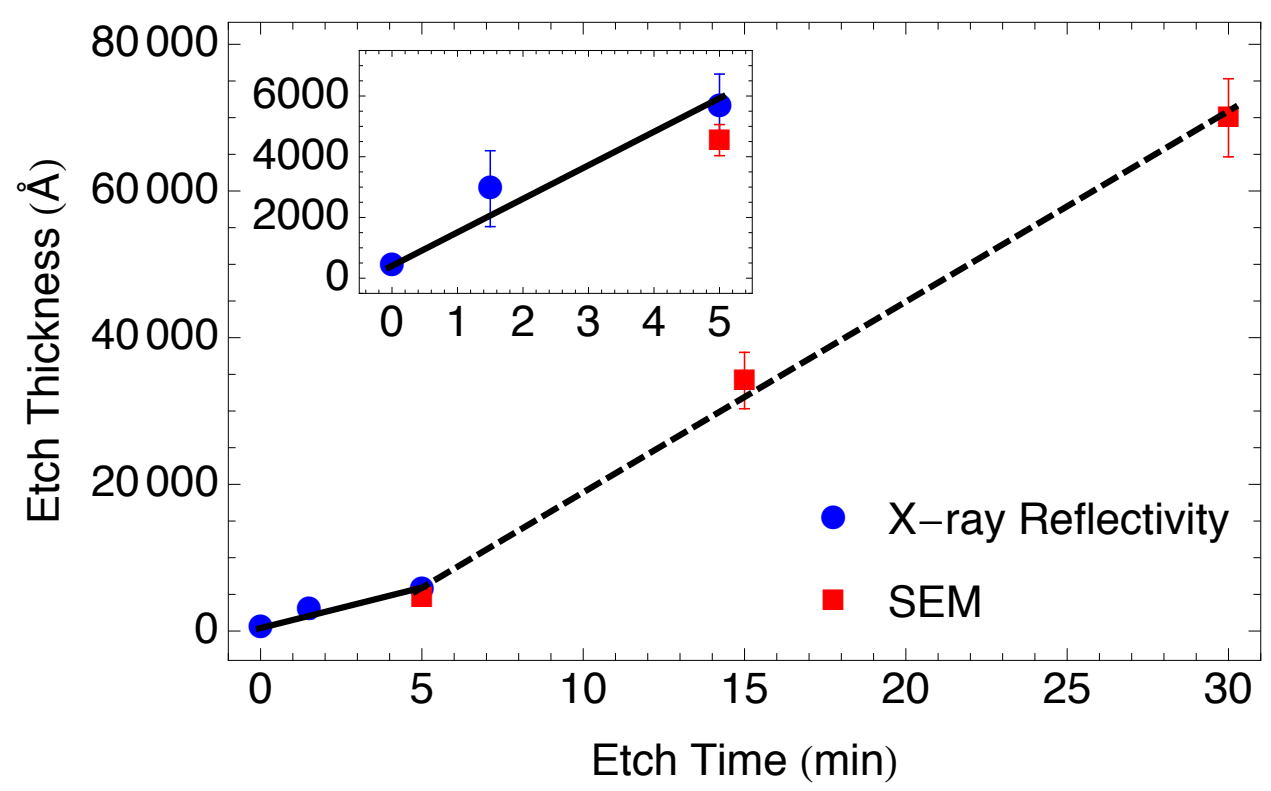

Figure 2.19: Etch thickness determined from SEM (red squares) and XRR (blue circles). The best fit lines indicate two etch rate regimes where the rate starts at $\sim 1000 \AA / \mathrm{min}$ (solid line) and then increases to $\sim 2600 \AA / \mathrm{min}$ (dashed line) after 5 min of etching. The inset shows a magnified view of the etch thicknesses for etch times of $5 \mathrm{~min}$ and under.

rate.

Figure 2.19 shows a summary of the NW lengths determined from both the SEM and the X-ray reflectivity measurements. Two etch-rate regimes are observed. In the early stages of etching, before the SiNWs are established, the rate starts at $\sim 1000$ $\AA /$ min and then increases to $\sim 2600 \AA /$ min after 5 min of etching, where the latter rate agrees with previous literature.[12] These different regimes exhibit different morphological features in the SEM images. The initial pitting of the Si surface, before the NWs are formed, can be seen in the SEM images of Fig. 2.3(a) and (b) whereas after 5 min of etching, established SiNWs are clearly observed in Fig. 2.3(c-h). It is interesting that the etch rate does not decrease with time even though the Ag progressively distributes along the wires with etch time, which suggests that only a small amount of $\mathrm{Ag}$ is required at the etch front in order to maintain the etching rate. 
The Ag composition profile determined from our study addresses etch mechanisms proposed in the literature. For example, because the 1-step fabrication method leaves significant amounts of $\mathrm{Ag}$ on the top surface, it has been proposed that the $\mathrm{Ag}$ acts as a mask protecting the covered Si from chemical etching.[30-32] However, our experimental results clearly show that a protecting mask is not required to form the NWs because very little Ag resides at the surface. Other studies using the 2step fabrication method, which is used in the present investigation, observed Ag concentrated at the etch front and attributed the etching to the Ag nanoparticles that are in direct contact with the Si. $[33,40]$ The latter explanation is supported by the present results that show the highest concentration of Ag at the etch front.

Our results, however, also suggest that the process is more complex than simply having Ag at the etch front because there is a considerable amount of Ag distributed along the NWs. The TEM images in Fig. 2.4(a) and (b) show faceted walls that are not pitted and the SEM images show no tapering of the NWs. Together, the XRR and microscopy results challenge the notion that $\mathrm{Si}$ that is in direct contact with $\mathrm{Ag}$ will be etched.[12, 33, 40, 47] The question which ultimately must be answered is why do the nanoparticles at the etch front propagate the etching while those along the wires appear to be ineffective at etching? Although the crystalline anisotropy of the etch rate is clearly important $-\{100\}$ is the preferred[37] etch direction for the low ratios of $[\mathrm{HF}] /\left[\mathrm{H}_{2} \mathrm{O}_{2}\right]$ used in this investigation - anisotropic etching alone is not sufficient to explain the results. For example, $\{100\}$ planes will be present at some point in time on the surface of the NWs and they would be etched. An additional process must, therefore, be active. An intriguing possibility could involve the surface curvature,[48] noting that the curvature of an etch pit has the opposite sign of the curvature of a wire surface. With Ag ions in solution and the highest ion density expected to be located relatively close to the Ag nanoparticle, the catalytic activity 
might be affected by surface curvature on relatively longer length-scales. While this idea is speculative, it is consistent with our experimental results. Clearly further work will be necessary.

In conclusion, using the unique ability of the X-ray specular reflectivity technique to determine buried composition profiles on the nanoscale we have been able to quantitatively show, for the first time, that the Ag nanoparticle etch catalyst is distributed along the length of the SiNWs. We note that the reflectivity technique works on rough surfaces as long as one can find a method to identify the specular component of the reflection. New information about the conservation of $\mathrm{Ag}$, the coarsening of the Ag nanoparticles as well as the abruptness of the interface at the etch front was obtained. The fact that the $\mathrm{Ag}$ is not localized at the surface or at the buried interface at the etch front provides new insight into the mechanism of the metal-assisted etching process. For example, the etching is highly directional and it apparently requires a relatively small amount of $\mathrm{Ag}$, while there appears to be a strong attraction of the Ag towards the etch front. In order to make further progress towards understanding the mechanism of metal-assisted etching of NWs, we suggest that future investigations focus on understanding the directionality of the etch rate as well as explore the role of surface curvature of the SiNWs and the metal-catalyst on the resulting NW morphology.

\subsection{Acknowledgments}

$\dagger$ Arendse and Cummings are acknowledged for contributing these sections.

Support is gratefully acknowledged from the National Science Foundation (USA) - DGE1069091, the National Research Foundation (RSA) - TTK14052167658, 76568, 92520, and 93212; and the University of Missouri/University of Western Cape Linkage 
Program. 


\section{Part II}

\section{Forbidden Bragg Reflections and Charge Density Distributions in Crystal Truncation Rods (CTRs)}




\section{Chapter 3}

\section{Introduction: Part II}

The distribution of atoms and charge on the atomic length scale in solids is pivotally important in determining all traits of a material including its crystal structure, electronic and vibrational properties, as well as all of the ancillary physical properties that depend on these, such as the optical, transport, and thermodynamic behavior. Furthermore, charge density distribution dictates many property characteristics of matter, such as diamagnetic susceptibility, multi-pole moments, electrostatic interaction energy, and non-linear optical susceptibilities, to name a few.[49] Therefore, it is not surprising that charge density distributions is important to many fields of science, including chemistry, physics, biology, and material science.[50] A variety of experimental techniques are used to study solid-state materials at the atomic length scale, examples include electron microscopy, X-ray photoelectron spectroscopy, and atomic force microscopy.[27] These tools are used to ascertain the structure of materials, infer bonding characteristics, and determine the distribution of atoms. However

these tools are limited in their ability to spatially resolve the surface and subsurface structure of a material at the atomic scale simultaneously.[41] X-ray scattering has 
the unique ability to not only measure the surface and bulk structure simultaneously, but is also capable of extracting spacial information throughout a wide range of length scales.

The conventional isotropic spherical treatment of the charge density distribution, also known as the independent-atom model (IAM), is frequently used to explain Xray scattering data and solve crystallographic structures.[51] However, this approach is inadequate for explaining the presences of forbidden Bragg reflections in our X-ray reflectivity measurements, nor is it able to describe the electron density distribution around an atom. In Chapter 5 we will introduce an non-spherical charge density distribution correction that will permit us to examine the interactions between X-ray scattering measurements and the non-spherical electron density distributions using a simple formalism. Our results demonstrate that with proper care, phase information can be extracted from the interference between the bulk forbidden Bragg reflection and the waves from the surface. Additionally, we show that the covalent bond density distribution can be extracted - for the first time - from a X-ray crystal truncation rod measurement.

To begin, it is useful to examine the real-space structure of the charge density distribution of the diamond crystal structure. Multiple models are available that incorporate the complexities of the distribution beyond the first-order spherical approximation. $[49,51]$ Dawson's multi-pole density formalism is a convenient approach to describe the deviation from spherical symmetry. For example, the atom-centered multi-polar functions of the Dawson formalism allows for convenient visualization of the system. Furthermore, it has been shown that it is sufficient at explaining experimental data. [52] Therefore it will be used here to discuss the non-spherical electron density distribution.

We acknowledge that other models have better agreement with experiential data 
due to their greater abundance of parameters, [51] but these are unnecessarily complex for the purposes here.

We will examine the charge density distribution of diamond crystal structures in real-space and reciprocal-space in Chapters 4 and 5, respectively. Chapter 5 will include the atomic form factor, structure factor, as well as the non-spherical charge density distribution correction factor. In Chapter 6 we will review previous experimental work on forbidden Bragg reflections and experimentally determined charge density distributions. Chapter 7 we will discuss forbidden Bragg reflections and charge density distributions in crystal truncation rods (CTRs).

Note: the term charge density is often defined as the sum of both the electron and nuclear densities in position spaces. Though, "charge density" and "electron density" are used interchangeably in the literature even when referring to the electron distribution on its own.[53] Therefore, both terms will be used interchangeably in this text. 


\section{Chapter 4}

\section{Charge Density Distribution in Real-Space}

\subsection{Introduction}

Here we discuss the non-spherical nature of the diamond crystal structure electron density distribution in real-space. Dawson's[54, 55] multi-pole charge density distribution is a convenient formalism for examining the nature of the charge density distribution. This approach involves deforming the conventional spherical charge density distribution with a combination of spherical harmonics. Therefore, this method allows us to examine the details of the covalent bonding distribution and the core deformation separately. 


\subsection{Charge Density Distribution}

\subsubsection{General Non-spherical Charge Density Distribution}

In the case of non-centrosymmetric atoms, such as in diamond crystal structures like Si and Ge, the total charge density distribution, $\rho(\vec{r})$, can be constructed generally in terms of centrosymmetric, $\rho_{c}(\vec{r})$, and asymmetric, $\rho_{a}(\vec{r})$, parts:

$$
\rho(\vec{r})=\rho_{c}(\vec{r})+\rho_{a}(\vec{r})
$$

where $\vec{r}$ is position about the atom. The details of each term are discussed for the diamond crystal structure below.

\subsubsection{Diamond Crystal Structure}

Figure 4.1(a) shows a schematic diagram of the diamond crystal structure cubic unit cell. In the this crystal structure, each atom has four nearest neighbors that

are arranged tetrahedrally, which corresponds to the Fd3m space group. [55] The structure type is centrosymmetric, but the atoms lack this property. This character is seen in the difference between the orientation of the two basis sites, as seen in Fig 4.1(b) and (c), which exhibit $\overline{4} 3 m$ site symmetry.

Tailoring the charge density distribution for the diamond crystal structure, Eq. (4.1) is expressed in terms of antisymmetric bonding, based on the $\overline{4} 3 m$ site symmetry, and the centrosymmetric term is expanded into two components in order to satisfy the $m 3 m$ site symmetry. The centrosymmetric terms include, the spherically symmetric part, $\bar{\rho}_{c}(r)$, and the centrosymmetric time-averaged shifted position part, $\delta \rho_{c}(\vec{r})$. The 


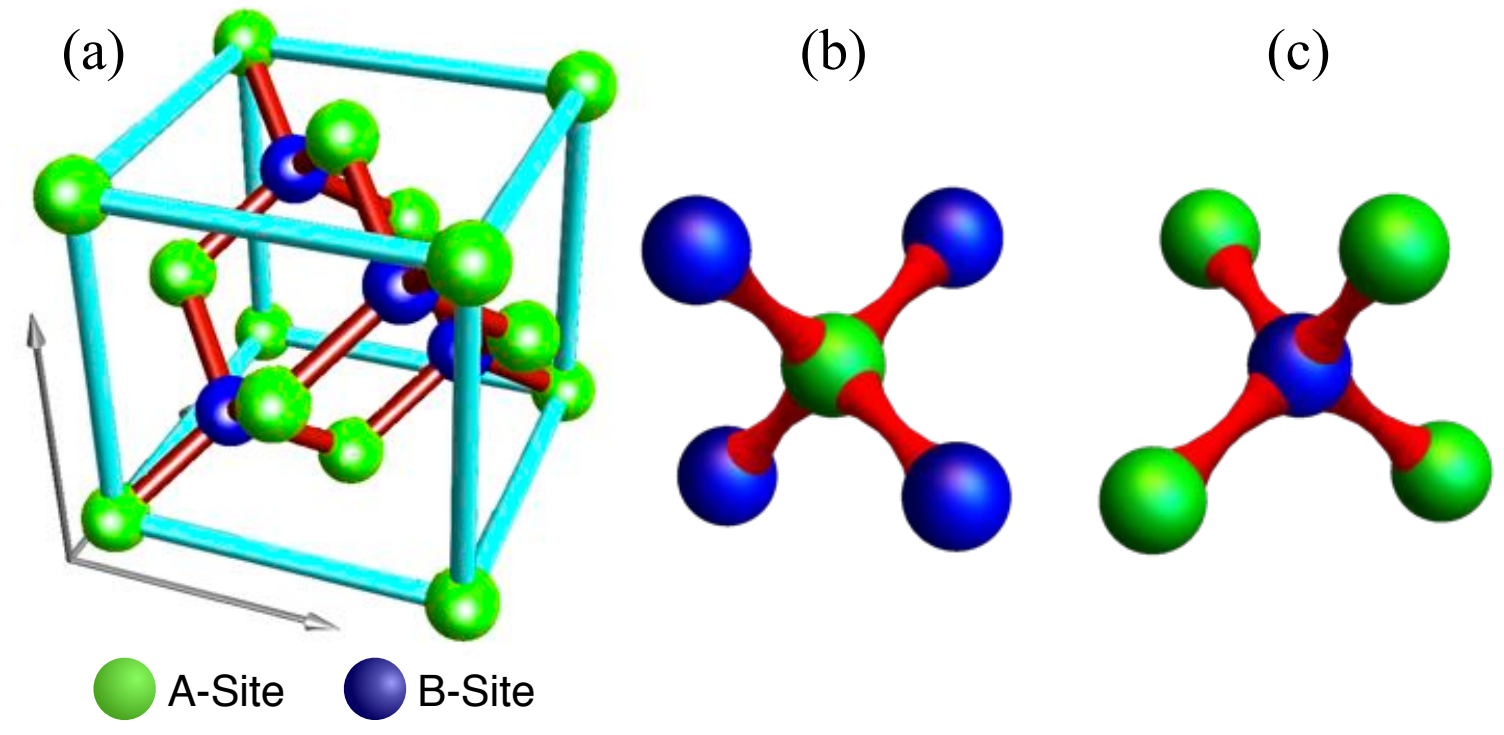

Figure 4.1: (a) Schematic diagram of the diamond crystal structure cubic unit cell. (b) and (c) show schematically the bonding configurations of the A and B basis sites, respectively. The A and B sites are identified with green and blue spheres, respectively.

charge density of an atom in the diamond crystal structure is written as[54]:

$$
\rho(\vec{r})=\bar{\rho}_{c}(r)+\delta \rho_{c}(\vec{r})+\rho_{a}(\vec{r}) .
$$

The non-spherical components do not contribute additional electrons or charge to the atom but rather redistribute the existing electron density distribution. Therefore, the expansion from Eq. (4.2) satisfies the following two condition[56]:

$$
\int \rho(\vec{r}) d \vec{r}=\int_{0}^{\infty} 4 \pi r^{2} \bar{\rho}_{c}(r) d r=Z
$$

where $Z$ is the atomic number, and

$$
\int \rho_{a}(\vec{r}) d \vec{r}=\int \delta \rho_{c}(\vec{r}) d \vec{r}=0
$$


The non-spherical charge density about the nucleus, from Eq. (4.2), can be expanded to include the sixth-order term $[54,55]$ :

$$
\begin{aligned}
\rho_{a}(\vec{r}) & =\rho_{a, 3}(\vec{r}), \\
\delta \rho_{c}(\vec{r}) & =\delta \rho_{c, 4}(\vec{r})+\delta \rho_{c, 6}(\vec{r}) .
\end{aligned}
$$

The fifth order asymmetric term is not allowed due to the $\overline{4} 3 m$ site symmetry[57]. Expressing the density in terms of Kubic Harmonics of Von der Lage and Bethe[55, 58] we have

$$
\begin{aligned}
\rho_{a, 3}(\vec{r}) & =F_{3}(r)\left(\frac{x y z}{r^{3}}\right) \\
\delta \rho_{c, 4}(\vec{r}) & =G_{4}(r)\left(\frac{x^{4}+y^{4}+z^{4}}{r^{4}}-\frac{3}{5}\right), \\
\delta \rho_{c, 6}(\vec{r}) & =G_{6}(r)\left(\frac{x^{2} y^{2} z^{2}}{r^{6}}+\frac{1}{22}\left(\frac{x^{4}+y^{4}+z^{4}}{r^{4}}-\frac{3}{5}\right)-\frac{1}{105}\right) .
\end{aligned}
$$

$x, y, z$ are real-space directions along the edges of a cubic unit cell. $K_{3, q m}$ and $L_{n, q m}$ are constants. The independent radial functions $F_{3}(r)$ and $G_{n}(r)$ are

$$
\begin{gathered}
F_{3}(r)=K_{3, q m} r^{q} e^{-\alpha r^{m}} \\
G_{n}(r)=L_{n, q m} r^{q} e^{-\alpha r^{m}}
\end{gathered}
$$

where $q$ and $m$ are constants with integer values, $q \neq 0$, and $\alpha$ is a parameter that adjusts the radial distribution of the charge density and has the units of $\AA^{-m}$.

It has been determined elsewhere[59] that the radial functions, Eq. (4.7), with $q=m=2$ give the best-fit to experimental data than when $q$ and $m$ are other integer values. Therefore, we use $q=m=2$ in our calculations. Furthermore, it has been determined elsewhere $[56,59]$ that including up to the $4^{\text {th }}$ order expansion 
Table 4.1: Previously determined[59] charge density distribution parameters.

\begin{tabular}{cccc} 
& $\alpha\left(\AA^{-2}\right)$ & $K_{3,22}$ & $L_{4,22}$ \\
\hline \hline Si & $0.970 \pm 0.005$ & $1.382 \pm 0.020$ & $-0.206 \pm 0.021$ \\
\hline
\end{tabular}

is sufficient for explaining experimental results from $\mathrm{C}$ and $\mathrm{Si}$.

It is helpful to examine the non-spherical charge density components using the three-dimensional plots shown in Fig. 4.2. Previously determined[59] Si values of $\alpha$, $K_{3,22}$, and $L_{4,22}$, seen in Table 4.1 , were used to render $\rho_{a, 3}$ and $\delta \rho_{c, 4}$. The values for $\delta \rho_{c, 6}$ are shown in arbitrary units due to the absence of experimental values for Si. The non-spherical charge density distributions are shown in two formats, where the plots on the left and right are three-dimensional density plots and complimentary cross-sectional contour plots, respectively.

The positive lobes are shown in red and yellow and the negative lobes are shown in blue and purple. Eq. (4.4) is satisfied in these plots. The positive and negative lobes alternate in $\rho_{a, 3}$. In $\delta \rho_{c, n}$, the density distributions of the positive and negative lobes are different, because they compensate for the uneven number of positive and negative lobes.

The spatial orientation of the electron density distributions shown in Fig. 4.2 correspond to the A-site atoms, while the B-site atoms are related by an inversion symmetry. The $\rho_{a, 3}$ positive lobes in (a) and (b) are oriented toward the nearest neighbor atoms. It can be seen that $\delta \rho_{c, n}$ are invariant to an inversion operation while $\rho_{a, 3}$ is not - this attribute will be exploited later in this text. The difference in densities between $\rho_{a, 3}$ and $\delta \rho_{c, 4}$ can be seen by the difference between the scale bars. 

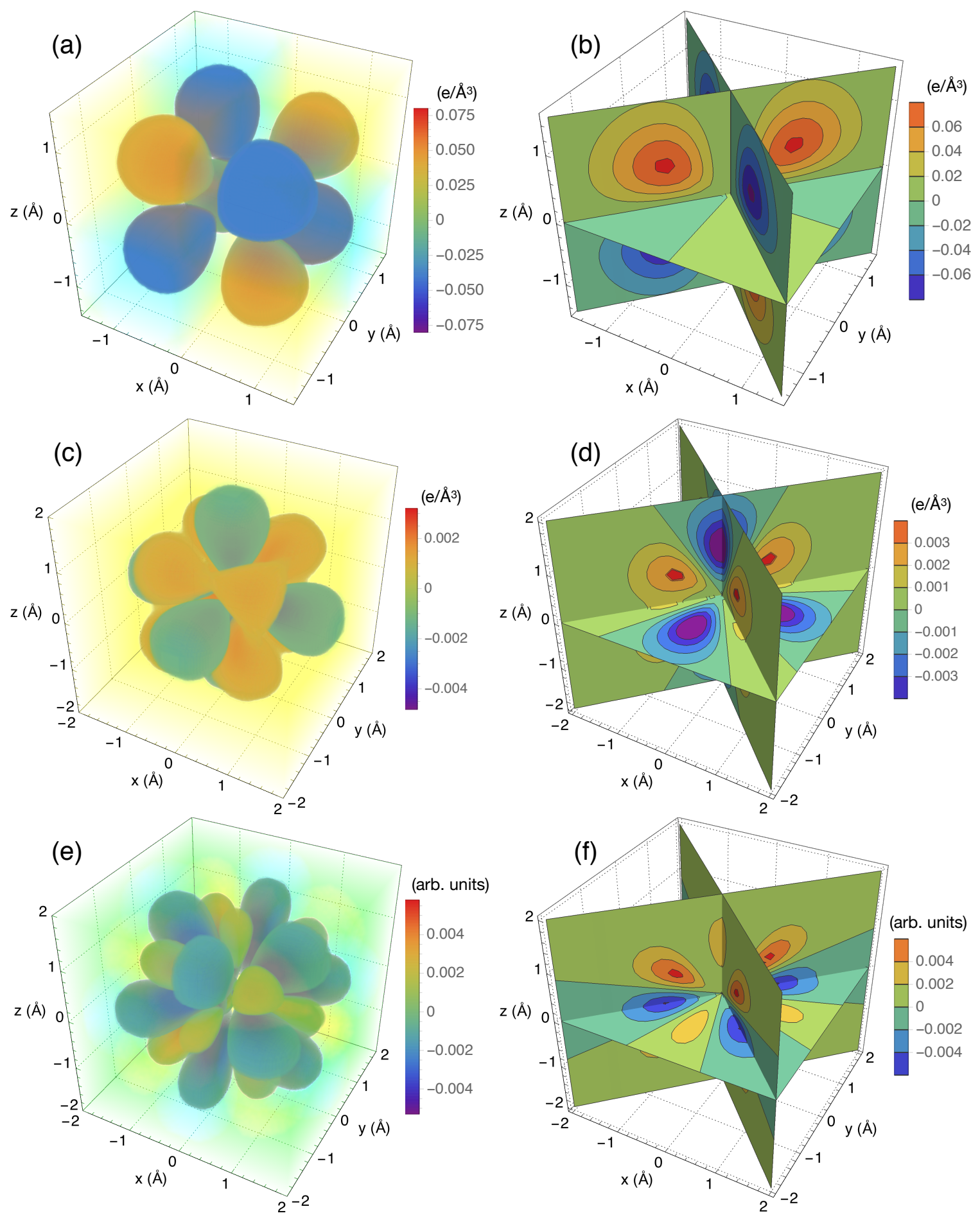

Figure 4.2: Non-spherical electron density distribution components of the A-site atoms (B-site is related by inversion symmetry). ((a) and (b)) are $\rho_{a, 3},((\mathrm{c})$ and (d)) are $\delta \rho_{c, 4}$, and $((\mathrm{e})$ and $(\mathrm{f}))$ are $\delta \rho_{c, 6}$. On the left and right are three-dimensional density plots and complimentary cross-sectional contour plots, respectively. 


\section{Chapter 5}

\section{Charge Density Distribution in Reciprocal-Space}

\section{$5.1 \quad$ Introduction}

In this chapter we examine the charge density distribution in reciprocal-space. We use the Dawson formalism[60] to aid in our inspection of the electron density distribution in diamond crystal structures. After the example model from Dawson we will introduce the Gamma-formalism, which modifies the conventional spherical charge density distribution model with a general non-spherical charge density distribution correction. It will allow us to examine the scattering behavior of non-spherical charge density distributions with a general formalism. For simplicity we will use the electron density distribution from the Dawson formalism to provide the details of the Gamma-formalism for the diamond crystal structure.

Before we get there, it is helpful to examine the non-spherical charge density distribution of the diamond crystal structure using Dawson's formalism. Section 5.2 will review the Dawson formalism, which was developed over the course of several 
publications[54-56, 60-63]. More specifically, Section 5.2.1 will discuss the reciprocalspace analogue of the real-space charge density distribution discussed in Chapter 4 . In Section 5.2.2 we will discuss how to correctly incorporate the anomalous scattering effects into the complex atomic form factor. In Sections 5.2.4 and 5.2.5 we will review the general temperature dependent structure factor and anharmonic thermal vibrations in the diamond crystal structure, respectively. The $\gamma$-formalism will be presented and discussed in Section 5.4.

The general structure factor is given in Section 5.2.3 and the structure factor for the diamond crystal structure is given in Section 5.3.2. $\mathrm{Si}(111)$ samples were used in our X-ray measurements and due to the orientation of the crystal structure relative to surface it is convenient use the hexagonal coordinate system when discussing our experimental results. The relationship between the cubic and hexagonal coordinate systems is given in Section 5.5.

The cubic coordinate system is used exclusively until Section 5.5. Then after, references to both coordinate systems are used, therefore subscripts " $C$ " and " $H$ " are used to differentiate between the cubic and hexagonal coordinate systems, respectively.

\subsection{Dawson Formalism}

\subsubsection{Atomic Form Factor}

The atomic form factor, $f$, is the reciprocal-space analogue of the real-space charge density distribution, $\rho$, discussed in Chapter 4 . The atomic form factor is determined by taking the Fourier transform of the atomic charge density distribution and is 
expressed as[60]

$$
f(\vec{Q})=\int \rho(\vec{r}) e^{-i \vec{Q} \cdot \vec{r}} d^{3} r
$$

where $\vec{r}$ is the position about the atom and $\vec{Q}$ is the wavevector transfer in reciprocalspace. $\vec{Q} \equiv \vec{k}_{2}-\vec{k}_{1}$, where $\vec{k}_{1}$ and $\vec{k}_{2}$ are the incident and outgoing wavevectors, respectively, and $|Q|=\frac{4 \pi}{\lambda} \sin \theta$, where $\lambda$ is the wavelength and $\theta$ is half the angle between the incident and outgoing wavevectors, a schematic diagram is seen in Fig. 2.6 inset.

The reciprocal-space analogue of Eq. (4.1) is the general atomic form factor for non-spherical charge density distributions and is expressed as[60]

$$
f(\vec{Q})=f_{c}(\vec{Q})+i f_{a}(\vec{Q})
$$

where $f_{c}(\vec{Q})$ and $f_{a}(\vec{Q})$ are the centrosymmetric and asymmetric components, respectively.

The reciprocal-space analogue of the non-spherical real-space charge density distribution of Eq. (4.2) expanded to the sixth-order term is[55]

$$
f(\vec{Q})=\bar{f}_{c}(Q)+\delta f_{c, 4}(\vec{Q})+\delta f_{c, 6}(\vec{Q})+i f_{a, 3}(\vec{Q})
$$

where $\bar{f}_{c}(Q)$ is the spherically symmetric component, $\delta f_{c, n}(\vec{Q})$ are the centrosymmetric time-averaged shifted position components, and $f_{a, 3}(\vec{Q})$ is the asymmetric component. The form factor components determined in terms of Fourier-Bessel transforms 
are[54]:

$$
\begin{aligned}
\bar{f}_{c}(Q) & =\int_{0}^{\infty} 4 \pi r^{2} \bar{\rho}_{c}(r) j_{0}(Q r) d r=\left\langle j_{0}(Q)\right\rangle, \\
f_{a, 3}(\vec{Q}) & =-A_{3} \int_{0}^{\infty} 4 \pi r^{2} F_{3}(r) j_{3}(Q r) d r=-A_{3} K_{3, q m}\left\langle j_{3}(Q)\right\rangle, \\
\delta f_{c, n}(\vec{Q}) & =B_{n} \int_{0}^{\infty} 4 \pi r^{2} G_{n}(r) j_{n}(Q r) d r=B_{n} L_{n, q m}\left\langle j_{n}(Q)\right\rangle,
\end{aligned}
$$

where $j_{n}(Q r)$ are spherical Bessel functions of the order $n,\left\langle j_{n}(Q)\right\rangle$ are the FourierBessel transform of the atomic density, $K_{3, q m}$ and $L_{n, q m}$ are constants from Eq. (4.7),

$$
\begin{aligned}
& A_{3}=\frac{h k l}{\left(h^{2}+k^{2}+l^{2}\right)^{3 / 2}}, \\
& B_{4}=\frac{h^{4}+k^{4}+l^{4}}{\left(h^{2}+k^{2}+l^{2}\right)^{2}}-\frac{3}{5}, \\
& B_{6}=\left(A_{3}\right)^{2}+\frac{1}{22} B_{4}-\frac{1}{105},
\end{aligned}
$$

are angular factors that are analogous to the Kubic Harmonics from Eq. (4.6), and $h k l$ are the Miller indices. Analytic expressions of the Fourier-Bessel transforms for different values of $q, m$, and $n$ are tabulated by Dawson[55].

The behavior of the $3^{r d}, 4^{\text {th }}$, and $6^{\text {th }}$ order Fourier-Bessel transforms of the atomic charge density, $\left\langle j_{n}(Q)\right\rangle$, verse $Q$ and $\alpha$ can be seen in Fig. 5.1. It can be seen that the peak positions along $Q$ are dependent on $\alpha$. Thereby $\alpha$ alters the radial dependence of the charge density distribution. For a monatomic crystal, Dawson[54] proposed that $\alpha$ can be approximated by setting $\alpha$ so that the maximum value of $F_{3}(r)$ occurs at the bond midpoint. The estimated $\alpha$ for $\mathrm{Si}$ is $0.723 \AA^{-2}$.[55] This value is then refined experimentally with X-ray scattering.

The directional dependence of the $A_{3}, B_{4}$, and $B_{6}$ angular factors are illustrated 


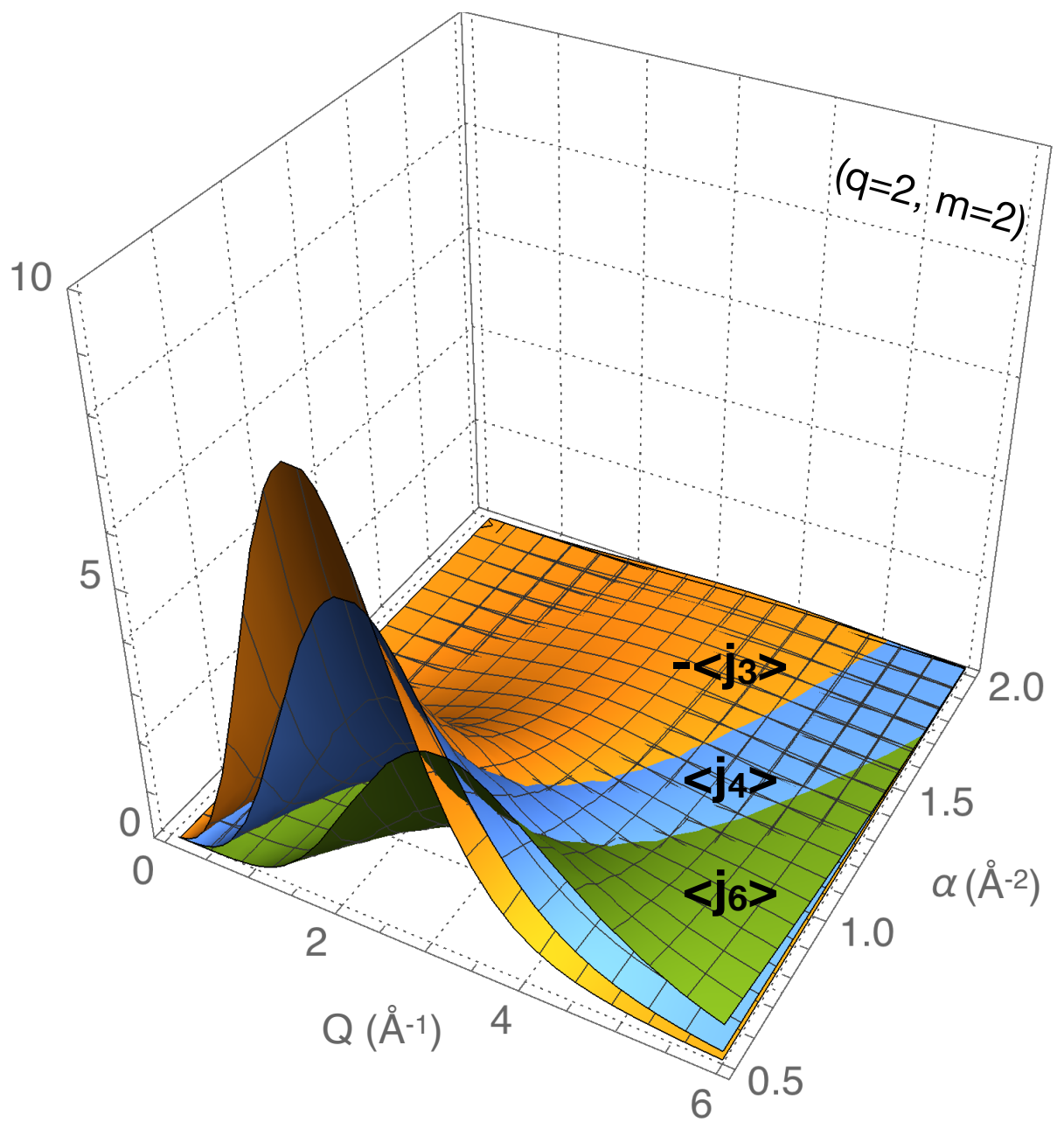

Figure 5.1: $3^{\text {rd }}, 4^{\text {th }}$, and $6^{\text {th }}$ order Fourier-Bessel transform of the atomic charge density, $\left\langle j_{n}(Q)\right\rangle$, verse $Q$ and $\alpha$. The constants are $q=2$ and $m=2$. 
in the three-dimensional plots in Fig. 5.2. Figure 5.2(a) and (b) show the directional dependence of $A_{3}$, where each set of four cones, positive (colored in red) and negative (colored in purple and blue), are oriented in a tetrahedral fashion, which provides the angular dependence of $f_{a, 3}$. The six positive cones of $B_{4}$ are shown in Fig. 5.2(c) and (d). These cones are oriented along the $h k l$ axises and dictate the angular nature of $\delta f_{c, 4}$ The directional dependence of $B_{6}$ is more complex that $A_{3}$ and $B_{4}$, where twenty cones occupy the same plotted region. These features reflect the higher angular frequency of the $6^{\text {th }}$ order atomic form factor term.

The values of several $A_{3}, B_{4}$, and $B_{6}$ angular factors for select reflections are listed in Table 5.1. The conventionally expected reflections are shown in regular text. The diamond structure specific $4 n+2$ forbidden reflections can be divided into two groups, which is reflected in the value of $A_{3}[55]$ and is identified in the table with either italics or bold text:

1. $A_{3}=0$ - when at least one of the Miller indices is zero, like (200) and (420) (in italics text) these are truly forbidden reflections

2. $A_{3} \neq 0$ - when none of the Miller indices are zero, like (222) and (622) (in bold text) Dawson referred to these as "unusual reflections"

Even though $B_{n} \neq 0$ for the truly forbidden reflections, the temperature factor extinguishes these contributions (see Section 5.2.5) and therefore these reflections remain truly forbidden.

Combining the angular dependence of $A_{3}$ and $B_{n}$, from Eq. (5.5) with the radial dependence of $\left\langle j_{n}(Q)\right\rangle$ we get the directional dependence, in three-dimensions, of the non-spherical atomic form factor components from Eq. (5.4). The three-dimensional nature of these components is shown in Fig. 5.3. Previously determined Si parameter values for $\alpha, K_{3,22}$, and $L_{3,22}$, seen in Table 4.1, were used to render these plots. The 

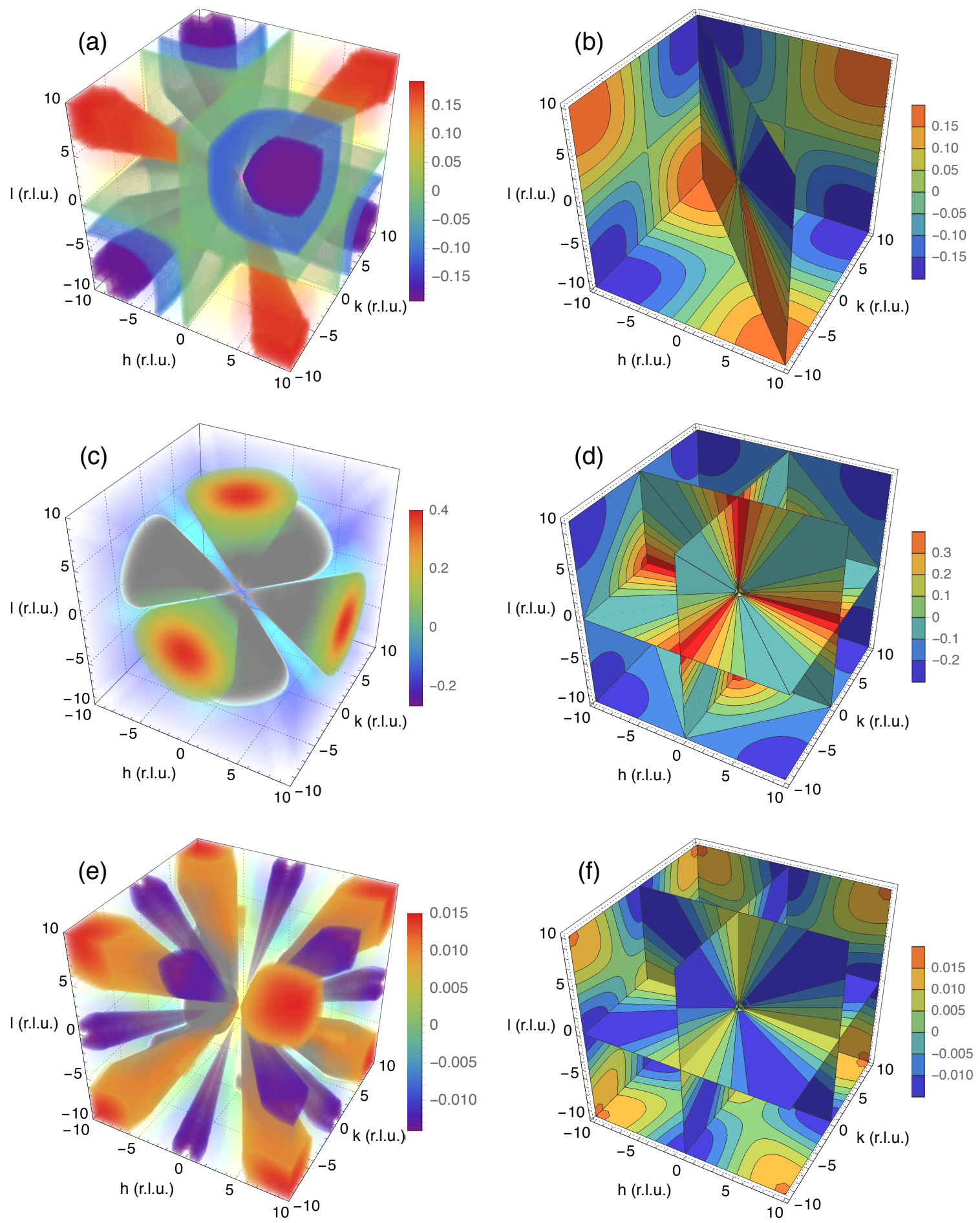

Figure 5.2: Three-dimensional plots of ((a) and (b)) $A_{3},\left((\mathrm{c})\right.$ and (d)) $B_{4}$, and ((e) and (f)) $B_{6}$. The plots on the left are three-dimensional density plots and complimentary cross-sectional contour plots are shown on the right. 
Table 5.1: Angular factors for the diamond crystal structure from Eq. (5.5), which are analogous to the Kubic Harmonics from Eq. (4.6).

\begin{tabular}{|c|c|c|c|c|c|}
\hline$h$ & $k$ & $l$ & $A_{3}$ & $B_{4}$ & $B_{6}$ \\
\hline 1 & 1 & 1 & 0.1925 & -0.2667 & 0.0154 \\
\hline 2 & 0 & 0 & 0.0000 & 0.4000 & 0.0087 \\
\hline 2 & 2 & 0 & 0.0000 & -0.1000 & -0.0141 \\
\hline 3 & 1 & 1 & 0.0822 & 0.0860 & 0.0011 \\
\hline 2 & 2 & 2 & 0.1925 & -0.2667 & 0.0154 \\
\hline 4 & 0 & 0 & 0.0000 & 0.4000 & 0.0087 \\
\hline 3 & 3 & 1 & 0.1087 & -0.1485 & -0.0045 \\
\hline 4 & 2 & 0 & 0.0000 & 0.0800 & -0.0059 \\
\hline 4 & 2 & 2 & 0.1361 & -0.1000 & 0.0044 \\
\hline 3 & 3 & 3 & 0.1925 & -0.2667 & 0.0154 \\
\hline 4 & 4 & 0 & 0.0000 & -0.1000 & -0.0141 \\
\hline 5 & 3 & 1 & 0.0724 & -0.0229 & -0.0053 \\
\hline 4 & 4 & 2 & 0.1481 & -0.1926 & 0.0037 \\
\hline 6 & 2 & 0 & 0.0000 & 0.2200 & 0.0005 \\
\hline 5 & 3 & 3 & 0.1596 & -0.1744 & 0.0080 \\
\hline 6 & 2 & 2 & 0.0822 & 0.0860 & 0.0011 \\
\hline 4 & 4 & 4 & 0.1925 & -0.2667 & 0.0154 \\
\hline
\end{tabular}


directional orientation corresponds to the A-site atoms, while the B-site atoms are related by an inversion symmetry. The lobes of the asymmetric bonding component, shown in (a) and (b), are oriented tetrahedrally, which is similar to the real-space charge density distribution seen in Fig. 4.2(a) and (b). Furthermore, it can also be seen that the general shape of the centro-symmetric form factor components are similar to the respective charge density distributions in Fig. 4.2.

\subsubsection{Anomalous Scattering Effects}

Accounting for the anomalous scattering effects the spherically symmetric component of the atomic form factor, from Eq. (5.3), is determined by

$$
\bar{f}_{c}(Q)=f_{c}^{0}(Q)+\Delta f^{\prime}(Q)+i \Delta f^{\prime \prime}(Q)
$$

where $f_{c}^{0}(Q)$ is the tabulated atomic form factor, $\Delta f^{\prime}(Q)$ and $\Delta f^{\prime \prime}(Q)$ are the in-phase and out-of-phase modifications of the tabulated atomic form factor, respectively, and are also referred to as the anomalous dispersion corrections. [60] Values of the atomic form factors and their anomalous dispersion corrections are tabulated in the International Tables for Crystallography, Volume C[64].

It is worth noting that the two types of complex scattering seen in Eq. (5.3) and (5.6) are the result of different effects.[60] The anomalous dispersion corrections in Eq. (5.6) are related to the inner electrons and are directionally invariant, therefore as a consequence the values are roughly independent of $Q$. Whereas in Eq. (5.3), the asymmetric scattering effects are related to the outer electrons and as a consequence is dependent on both the magnitude and direction of $\vec{Q}$.

As an example, lets include the anomalous scattering effects into Eq. (5.3) and then compare the total atomic form factor for when the direction of the scattering 

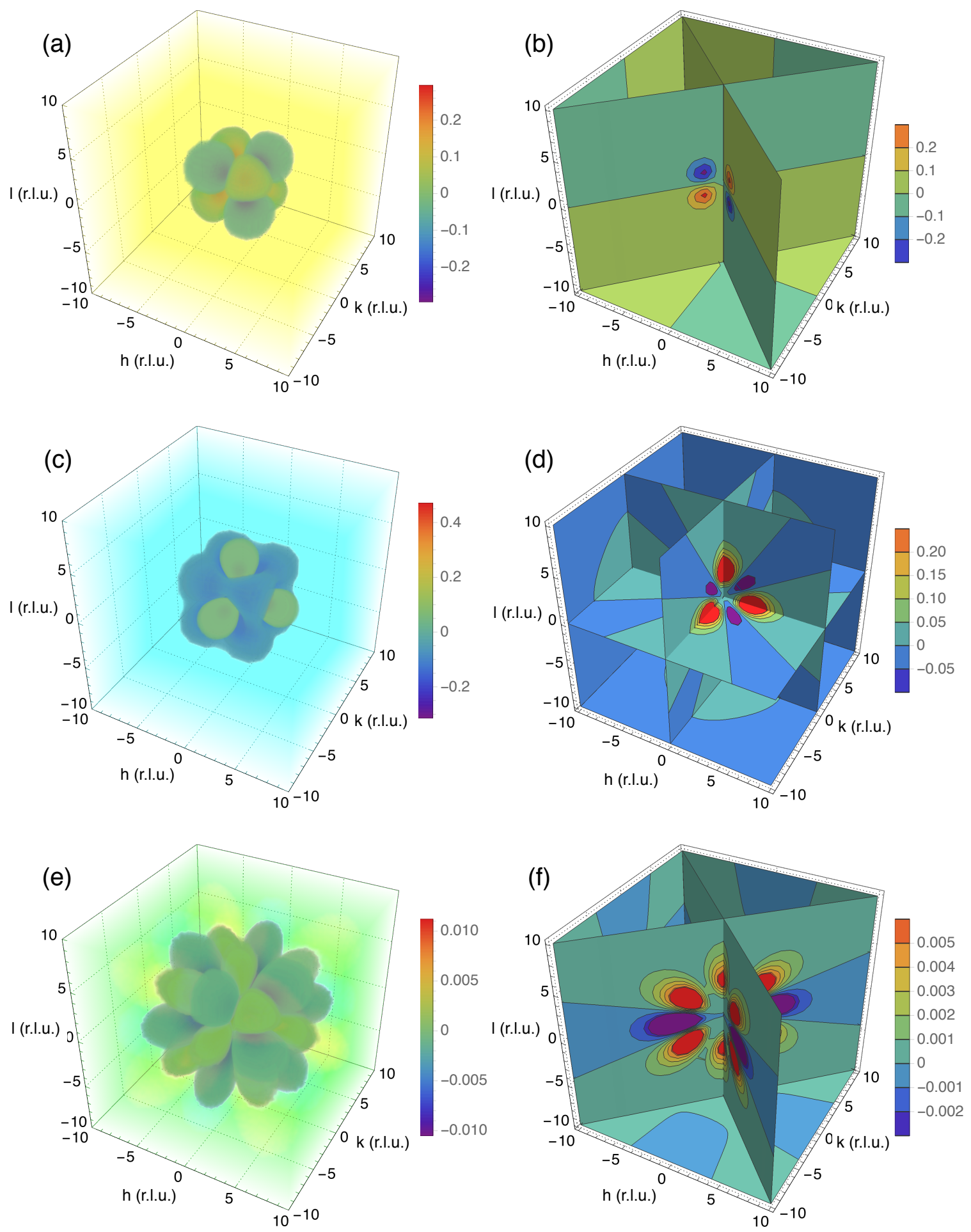

Figure 5.3: Three-dimensional plots of ((a) and (b)) $f_{a, 3},((\mathrm{c})$ and (d) $) \delta f_{c, 4}$, and ((e) and (f)) $\delta f_{c, 6}$. The plots on the left are three-dimensional density plots and complimentary cross-sectional contour plots are on the right. 
vector is reversed:

$$
\begin{aligned}
f(\vec{Q}) & =\left(f_{c}^{0}(Q)+\Delta f^{\prime}(Q)+i \Delta f^{\prime \prime}(Q)\right)+\delta f_{c, n}(\vec{Q})+i f_{a, 3}(\vec{Q}), \\
f(-\vec{Q}) & =\left(f_{c}^{0}(Q)+\Delta f^{\prime}(Q)+i \Delta f^{\prime \prime}(Q)\right)-\delta f_{c, n}(\vec{Q})-i f_{a, 3}(\vec{Q})
\end{aligned}
$$

It can be seen that the spherically symmetric and anomalous scattering terms, $\Delta f^{\prime}(Q)$ and $\Delta f^{\prime \prime}(Q)$, are directionally invariant and only depend on the scalar $Q$, whereas $\delta f_{c, n}(\vec{Q})$ and $f_{a, 3}(\vec{Q})$, from Eq. (5.3), are dependent on the vector $\vec{Q}$.

\subsubsection{Structure Factor}

The structure factor can be thought of as the collection of the independent atomic form factors in an ordered configuration, such as in a lattice. Where the atomic form factor describes the electron density around an atom. The structure factor describes the position density of atoms and therefore the collective position density of the electrons in the crystal. Or in other words, the convolution of the atomic form factor and the lattice.

The structure factor formalism developed by Dawson[60] is briefly reviewed below. The structure factor is determined by

$$
F(\vec{Q})=\sum_{l}^{\text {Unit cell }} f_{l}(\vec{Q}) e^{-i \vec{Q} \cdot \vec{r}_{l}}
$$

where $f_{l}(\vec{Q})$ is the atomic form factor for the $l^{\text {th }}$ atom located at $\vec{r}_{l}$ in the unit cell. 


\subsubsection{Temperature Dependent Structure Factor}

The effect of thermal vibrations on a charge density distribution can be thought of as the convolution of a static charge density distribution, $\rho(\vec{r})$, and a probability distribution that acts as a nuclear smearing function, $P(\vec{r})$, where $\langle\rho(\vec{r})\rangle=\rho(\vec{r}) * P(\vec{r}) \cdot[65]$ Then by taking the Fourier transform of the thermally smeared charge density distribution we can express the temperature dependent form factor as $\langle f(\vec{Q})\rangle=f(\vec{Q}) T(\vec{Q})$. Therefore the structure factor from Eq. (5.8) is rewritten as[51, 60]

$$
\begin{aligned}
F^{\prime}(\vec{Q}) & =\sum_{l}^{\text {Unit cell }}\left\langle f_{l}(\vec{Q})\right\rangle e^{-i \vec{Q} \cdot \vec{r}_{l}} \\
& =\sum_{l}^{\text {Unit cell }} f_{l}(\vec{Q}) T_{l}(\vec{Q}) e^{-i \vec{Q} \cdot \vec{r}_{l}}
\end{aligned}
$$

to account for the temperature dependence of the $l^{\text {th }}$ atom.

It is often sufficient to describe the thermal vibrations in an isotropic quadratic potential. The corresponding temperature factor is the Thermal Debye Waller Factor, which is expressed as[60]

$$
T_{c}=e^{-\frac{Q^{2} k_{B} T}{2 \alpha_{t}}}
$$

where $k_{B}$ is the Boltzman constant, $T$ is the absolute temperature, and $\alpha_{t}$ is the harmonic force constant as defined below.

However, when the potential is anisotropic $T_{c}$ is no longer adequate to describe the thermal vibrations. This is the case for the diamond crystal structure, where a consequence of its $\overline{4} 3 \mathrm{~m}$ site symmetry is anharmonic thermal vibrations. We will discuss a commonly used model for describing the anharmonic thermal vibrations of an atom in the diamond crystal structure in Section 5.2.5. 


\subsubsection{Anharmonic Temperature Factor: One-Particle Poten- tial model}

Multiple models are used to explain anharmonic thermal behavior.[51] It is convenient to use the One-Particle Potential model due to to its relative simplicity and because it is generally consistent with previous experimental results[66] for $\mathrm{Si}$ and Ge. This model was determined[62] and discussed[51] elsewhere and it is briefly summarized below.

The probability distribution of a particle located in a potential, $V(r)$, is expressed as $[51,62]$

$$
P(r)=N e^{\frac{-V(r)}{k_{B} T}}
$$

where $T$ is the absolute temperature and $N$ is the normalization constant that is defined by

$$
\int P(r) d r=1
$$

Using Dawson's[62] single-atom Einstein potential expanded to the third-order antisymmetric term for a tetrahedrally bonded atom is expressed as

$$
V(r)=V_{0}+\frac{1}{2} \alpha_{t}\left(x^{2}+y^{2}+z^{2}\right)+\beta_{t} x y z
$$

where $\beta_{t}$ is the anharmonic force constant. The harmonic force constant is determined by [59]

$$
\alpha_{t}=\frac{4 \pi^{2} m k_{B}^{2} \Theta_{M}^{2}}{3 h^{2}}
$$

where $m$ is the atomic mass, $\Theta_{M}$ is the Debye temperature, and $h$ is the Planck

constant. $\beta_{t}$ can be estimated from the thermal expansion coefficient or determined from the neutron intensity of the (222) reflection. [59] Previously determined[68] values of $\alpha_{t}$ and $\beta_{t}$ for Si are shown in Table 5.2. 
Table 5.2: Previously determined[68] values of the temperature factor parameters for Si.

\begin{tabular}{ccc} 
& $\alpha_{t}\left(\operatorname{erg} \AA^{-2}\right)$ & $\beta_{t}\left(\operatorname{erg} \AA^{-3}\right)$ \\
\hline \hline Si & $7.85 \times 10^{-12}$ & $5.0 \times 10^{-12}$ \\
\hline
\end{tabular}

Plugging the single-atom potential, Eq. (5.13), into the probability distribution, Eq. (5.11), we have

$$
P(r)=N e^{-\frac{\frac{1}{2} \alpha_{t}\left(x^{2}+y^{2}+z^{2}\right)+\beta_{t} x y z}{k_{B} T}} .
$$

$V_{0}$ is omitted because it does not affect the probability distribution. Using the approximation $e^{-x}=1-x$ for the cubic term, we get

$$
P(r)=N e^{-\frac{\frac{1}{2} \alpha_{t}\left(x^{2}+y^{2}+z^{2}\right)}{k_{B} T}}\left(1-\frac{\beta_{t} x y z}{k_{B} T}\right) .
$$

Considering that the temperature factor, $T(\vec{Q})$, is the Fourier transform of the probability distribution, the temperature factor for an atom in the diamond crystal structure is expressed as

$$
T(\vec{Q})=e^{-\frac{Q^{2} k_{B} T}{2 \alpha_{t}}}\left(1-i\left(k_{B} T\right)^{2}\left(\frac{2 \pi}{\alpha_{t}}\right)^{3} \frac{\beta_{t}}{V} h k l\right)
$$

where $V$ is the volume of the unit cell and $h k l$ are the Miller indices. The temperature factor can also be expressed generally in terms of its centrosymmetric and asymmetric components, $[67]$

$$
T(\vec{Q})=T_{c}(Q)+i T_{a}(\vec{Q})
$$


where $T_{c}$ is the Thermal Debye Waller Factor and $T_{a}$ is the anharmonic correction factor where[68]

$$
T_{a}=-T_{c}\left(k_{B} T\right)^{2}\left(\frac{2 \pi}{\alpha_{t}}\right)^{3} \frac{\beta_{t}}{V} h k l .
$$

Notice the sign of Eq. (5.19), it is the opposite of $f(\vec{Q})$, therefore $T_{a}$ will act in the opposite direction of $f(\vec{Q})$.

\subsection{Scattering From the Diamond Crystal Struc- ture}

In a scattering event, the observed X-ray intensity is proportional to the modulus square of the scattering amplitude,[69]

$$
I(\vec{Q}) \propto|A(\vec{Q})|^{2}
$$

The amplitude, $A(\vec{Q})$, of the X-rays scattering from atoms in a crystalline structure can be determined by the structure factor, $F(\vec{Q})$, of the material.

The diamond crystal structure can be described as two interpenetrating facecentered cubic (FCC) structures that are offset by $\frac{1}{4}$ along the body diagonal. Therefore, it is convenient to account for the atoms in terms of a FCC lattice. The position

of the atoms in the crystal can be organized as $\vec{r}_{l}=\vec{R}_{n}+\vec{r}_{j}$, where $\vec{R}_{n}$ is the lattice vector and $\vec{r}_{j}$ is the position of the $j$ th atom within a unit cell. The scattering amplitude in terms of these components, including the unit cell structure factor from 
Eq. (5.8) is:[42]

$$
\begin{aligned}
A(\vec{Q}) & =\sum_{\vec{R}_{n}+\vec{r}_{j}}^{\text {All atoms }} f_{l}(\vec{Q}) e^{-i \vec{Q} \cdot\left(\vec{R}_{n}+\vec{r}_{j}\right)} \\
& =\sum_{n}^{\text {Lattice }} e^{-i \vec{Q} \cdot \vec{R}_{n}} \times \sum_{j}^{\text {Unit cell }} f_{j}(\vec{Q}) e^{-i \vec{Q} \cdot \vec{r}_{j}}
\end{aligned}
$$

The first sum is of the FCC lattice sites and the second is the unit cell structure factor from Eq. (5.8), which includes two atoms, one at each basis site.

\subsubsection{Face-Centered Cubic Structure}

The cubic FCC lattice is shown in Fig. 5.4(a) where the cubic unit cell consists of four lattice sites located at $(0,0,0),\left(\frac{a}{2}, \frac{a}{2}, 0\right),\left(\frac{a}{2}, 0, \frac{a}{2}\right)$, and $\left(0, \frac{a}{2}, \frac{a}{2}\right)$, where $a$ is the lattice constant. Therefore, the corresponding the structure factor, and also the first sum from Eq. (5.21), is[69]

$$
F_{F C C}=1+e^{-i \pi(H+K)}+e^{-i \pi(H+L)}+e^{-i \pi(K+L)}
$$

where $H, K, L$ are the Miller indices in reciprocal-space, the wavevector transfer is $\vec{Q}=\frac{2 \pi}{a}(H \hat{x}+K \hat{y}+L \hat{z})$, and $\hat{x}, \hat{y}, \hat{z}$ are the real-space unit vectors. Evaluating the structure factor we find that [69]

$$
F_{F C C}=\left\{\begin{array}{cc}
4 & \text { when } H, K, L \text { are all odd or all even } \\
0 & \text { when } H, K, L \text { are mixed }
\end{array}\right.
$$

According to the selection rules it can be seen that the (111) reflection will be observed while the (100) reflection is forbidden due to the vanishing structure factor when the Miller indices are mixed. 

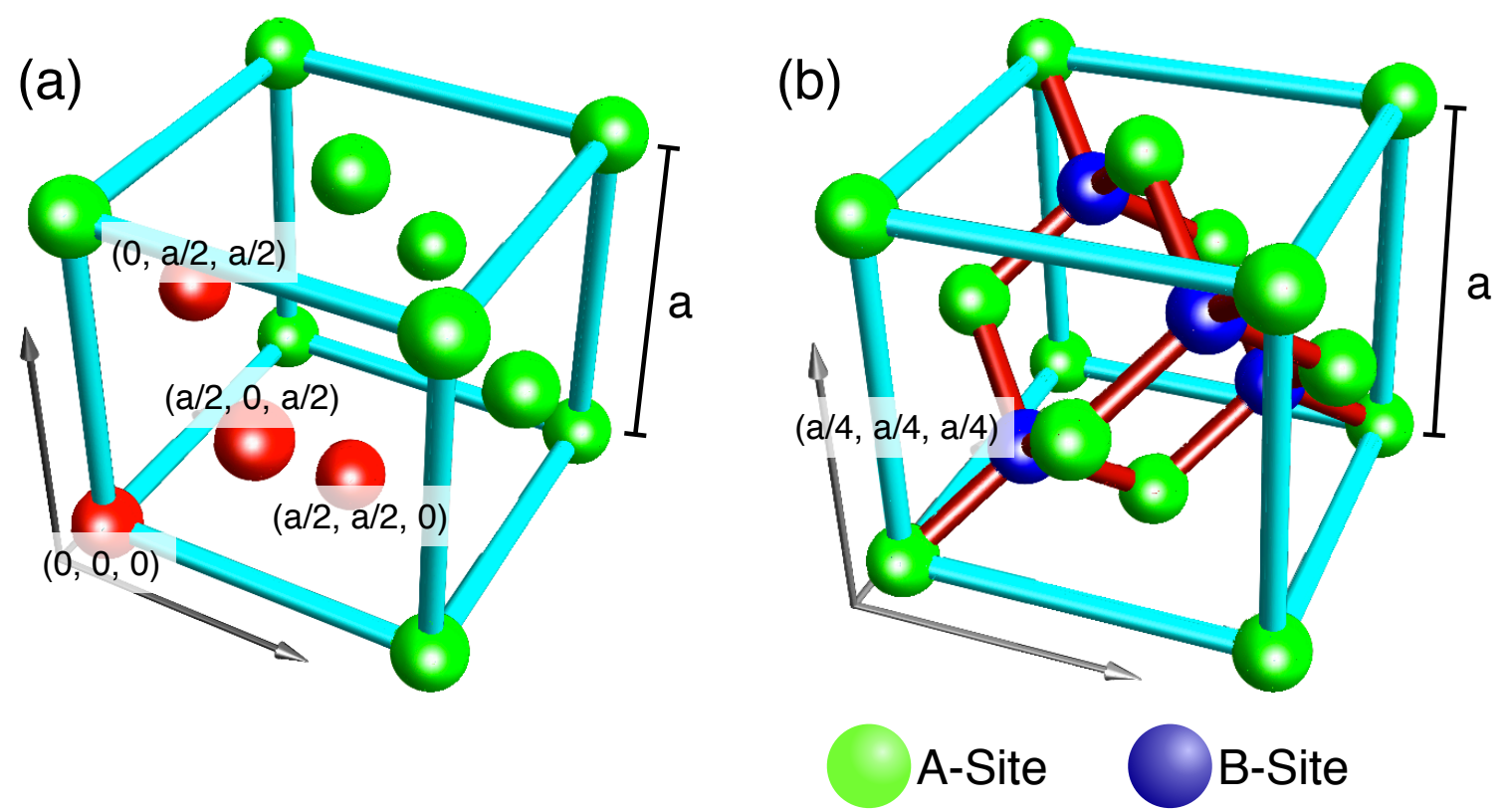

Figure 5.4: (a) Schematic of a face-centered cubic lattice. The four atoms that make up the cubic unit cell are shown in red. The cubic lattice constant, $a$, is shown. (b) Schematic of a cubic diamond-structure lattice. The B-sites are translated $\frac{1}{4}$ along the body diagonal from the A-sites.

\subsubsection{Diamond Crystal Structure}

Figure 5.4(b) shows a schematic diagram of the diamond crystal structure. Considering that its structure can be described as two interpenetrating FCCs, the structure factor can be determined by the convolution of an unit cell, consisting of an A-site and B-site atoms, and a FCC lattice. The A-B site unit cell will be referred to as the diamond double-layer (DL) unit cell, where the B-sites are translated from the A-sites by $\left(\frac{a}{4}, \frac{a}{4}, \frac{a}{4}\right)$ and the unit cell structure factor is expressed as [42]

$$
F_{D L}=f^{A}(\vec{Q})+f^{B}(\vec{Q}) e^{-i \frac{\pi}{2}(H+K+L)}
$$

where $f^{A}(\vec{Q})$ and $f^{B}(\vec{Q})$ are the atomic form factors of the A- and B-sites, respectively. Therefore, the structure factor of the diamond crystal structure in terms of the FCC 
lattice, from Eq. (5.22), and the the DL unit cell, Eq. (5.24) is[42]

$$
F_{D i a}=F_{F C C}\left[f^{A}(\vec{Q})+f^{B}(\vec{Q}) e^{-i \frac{\pi}{2}(H+K+L)}\right]
$$

The basis sites have tetrahedral symmetry (see Fig. 4.1(b) and (c)) and the A$\mathrm{B}$ sites are related by a point inversion, where the nearest neighbors and covalent bonds of the A-sites are oriented in opposite directions to those of the B-sites. The relation of the basis site charge density distributions can therefore be expressed as $\rho^{A}(\vec{r})=\rho^{B}(-\vec{r})$. Fourier transforming the charge density distribution we see that the atomic form factors are complex conjugates of each other, $f^{B}(\vec{Q})=f^{A}(\vec{Q})^{*}$, which is due to basis site inversion symmetry.

The second basis in the diamond-structure modifies the allowed FCC reflections from Eq. (5.23), by the selection rules of the double-layer structure factor, $F_{D L}$. Evaluating Eq. (5.25) we find that

$F_{D i a}=\left\{\begin{array}{c}\text { when } H, K, L \text { are: } \\ 4 \quad \text { all odd or even } \quad \times\left\{\begin{array}{l}f^{A}(\vec{Q})+f^{B}(\vec{Q}) \\ f^{A}(\vec{Q}) \pm i f^{B}(\vec{Q}) \\ 0 \quad \text { mixed }\end{array} \quad \text { when }(H+K+L)=\text { odd }\right. \\ f^{A}(\vec{Q})-f^{B}(\vec{Q}) \quad \text { when }(H+K+L)=4 n+2\end{array}\right.$

where $n$ is an integer.

When the A and B sites are identical, as when the charge is spherical, the electron density distributions and the form factors are indistinguishable, where $\rho^{A}(\vec{r})=\rho^{B}(\vec{r})$ and $f(\vec{Q})=f^{B}(\vec{Q})=f^{A}(\vec{Q})$, the $4 n+2$ reflections will be extinguished. It is worth noting the difference in origin between the two types of forbidden reflections in the diamond-structure. The FCC lattice structure extinguishes the reflections when the 
Miller indices are mixed. Whereas the $4 n+2$ reflections are due to the additional symmetry that is introduced from the second basis in the diamond-structure - these are diamond crystal structure specific forbidden Bragg reflections. These forbidden Bragg reflections are of interest because, as we saw in Chapter 4, the charge density is not entirely spherically distributed and therefore not all of the $4 n+2$ reflections are extinguished. We will discuss how to handle this situation below.

\subsection{Gamma-Formalism}

Here we introduce the Gamma-Formalism, which corrects the conventional spherical charge density model for the non-spherical charge density distributions. As an example we use the Dawson formalism to calculate the details of the correction and inspect the behavior of the non-spherical charge distributions.

\subsubsection{General Charge Density Distribution Correction Fac- tor}

Only a minor amount of the charge density distribution deviates from being spherically symmetric.[51] Therefore it is often sufficient to assume spherical symmetry and use the anomalous dispersion corrected tabular atomic form factor, $\bar{f}_{c}$, from Eq. (5.6) and the Thermal Debye Waller factor, $T_{c}$, from Eq. (5.10), when calculating the temperature dependent structure factor from Eq. (5.9), for example:

$$
F_{c}^{\prime}(\vec{Q})=\sum_{l}^{\text {Unit cell }} \bar{f}_{c, l}(Q) T_{c, l}(Q) e^{-i \vec{Q} \cdot \vec{r}_{l}}
$$

This is referred to as the Independent Atom Model (IAM). However, this approach,

as noted by W. H. Bragg,[70] dose not account for small perturbations from spherical 
symmetry that permit the observation of unexpected reflections.

We account for the details of the non-spherical charge density distribution by introducing a simple formalism that amends Eq. (5.27) with a non-spherical charge density distribution correction factor, $\gamma(\vec{Q})$. As a result, the product of the complex atomic form factors and temperature factors from Eq. (5.2) and (5.18), respectively, can be written in terms of the conventional spherically symmetric terms, from Eq. (5.27), and the correction factor:

$$
f(\vec{Q}) T(\vec{Q})=\bar{f}_{c}(Q) T_{c}(Q) \gamma(\vec{Q})
$$

$\gamma(\vec{Q})$ is complex and its details can be ascertained from atomic charge density distribution models.

We use the Dawson formalism, discussed above, to calculate the details of $\gamma(\vec{Q})$ because of the simplicity of this model and that it has been shown to be generally sufficient for describing the charge density distribution in the diamond crystal structure[57]. To calculate $\gamma(\vec{Q})$ we start with the general form of the temperature dependent atomic form factor in the Dawson formalism:[67]

$$
f(\vec{Q}) T(\vec{Q})=\left(f_{c}+i f_{a}\right)\left(T_{c}+i T_{a}\right)
$$

which is the product of Eq. (5.2) and (5.18).

Expressing Eq. (5.29) in terms of $\gamma(\vec{Q})$, we have

$$
\begin{aligned}
f(\vec{Q}) T(\vec{Q}) & =\bar{f}_{c} T_{c}\left[\left(\frac{f_{c}}{\bar{f}_{c}}-\frac{f_{a} T_{a}}{\bar{f}_{c} T_{c}}\right)+i\left(\frac{f_{a}}{\bar{f}_{c}}+\frac{f_{c} T_{a}}{\bar{f}_{c} T_{c}}\right)\right] \\
& =\bar{f}_{c} T_{c} \gamma(\vec{Q}) .
\end{aligned}
$$


Therefore, $\gamma(\vec{Q})$ calculated with the Dawson formalism is

$$
\gamma(\vec{Q})=\left(\frac{f_{c}}{\bar{f}_{c}}-\frac{f_{a} T_{a}}{\bar{f}_{c} T_{c}}\right)+i\left(\frac{f_{a}}{\bar{f}_{c}}+\frac{f_{c} T_{a}}{\bar{f}_{c} T_{c}}\right)
$$

where

$$
\begin{aligned}
& \frac{f_{c}}{\bar{f}_{c}}-\frac{f_{a} T_{a}}{\bar{f}_{c} T_{c}}=\operatorname{Re}(\gamma(\vec{Q})) \\
& \frac{f_{a}}{\bar{f}_{c}}+\frac{f_{c} T_{a}}{\bar{f}_{c} T_{c}}=\operatorname{Im}(\gamma(\vec{Q})) .
\end{aligned}
$$

Written in this form it can be seen that $\operatorname{Re}(\gamma(\vec{Q}))$ and $\operatorname{Im}(\gamma(\vec{Q}))$ represent the centrosymmetric and anisotropic components of the non-spherical charge density distribution correction, respectively. "Charge anisotropy" refers to anisotropic component of the non-spherical charge density distribution and is represented by $\operatorname{Im}(\gamma(\vec{Q})) . \gamma(\vec{Q})$ deforms the charge density distribution and is unit-less. $\operatorname{Re}(\gamma(\vec{Q}))$ and $\operatorname{Im}(\gamma(\vec{Q}))$ consolidate the centrosymmetric and anisotropic charge density corrections, respectively, into a single term.

It is convenient to examine the net non-spherical density distribution using the $\gamma$-formalism. An example of its utility is that the direction and magnitude of the net charge anisotropy can be directly identified from $\operatorname{Im}(\gamma(\vec{Q}))$. The sign of $\operatorname{Im}(\gamma(\vec{Q}))$ indicates the direction of the net anisotropic charge density distribution, where:

1. $\operatorname{Im}(\gamma(\vec{Q}))<0$ - the charge anisotropy is dominated by the asymmetric bonding charge from the accumulation of charge between the nearest neighbor atoms.

2. $\operatorname{Im}(\gamma(\vec{Q}))>0$ - the charge anisotropy is dominated by anharmonic thermal vibrations from the accumulation of charge toward the void opposite the bonds.

3. $\operatorname{Im}(\gamma(\vec{Q}))=0$ - indicates that the charge density distribution is symmetric. 
$\operatorname{Im}(\gamma(\vec{Q}))=0$ does not imply that $\operatorname{Re}(\gamma(\vec{Q}))=1 . \operatorname{Re}(\gamma(\vec{Q})) \neq 1$ indicates that the charge density distribution is only centrosymmetric in nature, as an example where $\rho(\vec{r})=\bar{\rho}_{c}(r)+\delta \rho_{c, n}(\vec{r})$. Lastly, $|\operatorname{Im}(\gamma(\vec{Q}))|$ is proportional to the magnitude of the net anisotropic charge density distribution.

\subsubsection{Non-spherical Charge Density Distribution Correction for Silicon}

In this section we will inspect the behavior of $\gamma(\vec{Q})$, from Eq. (5.31), using silicon as an example. The temperature factor from Eq. (5.17) was calculated using previously determined parameter values given in Table 5.2. The Boltzmann constant is taken as $1.38 \times 10^{-16} \mathrm{erg} \mathrm{K}^{-1}$ and $5.431 \AA$ is the Si lattice constant.[71] $f_{c}$ and $f_{a}$, from Eq. (5.4), were calculated using previously determined $\alpha, K_{3,22}$, and $L_{4,22}$ values seen in Table 4.1.

\section{Three-dimensional Behavior}

A useful way to survey the features of the non-spherical charge density distribution correction is with three-dimensional plots of $\gamma^{S i}(h k l)$ shown in Fig. 5.5. Figure 5.5(a) and (b) show the real parts, (c) and (d) show the imaginary parts, and (e) and (f) show the magnitude of the correction factor, all at $300 \mathrm{~K}$. The plots on the left are three-dimensional density plots and complimentary cross-sectional contour plots are shown on the right for visual clarity. The features of the imaginary and real parts of $\gamma^{S i}(h k l)$ appear to be similar to the three-dimensional distribution of $f_{a, 3}$ and $\delta f_{c, 4}$ seen in Fig. 5.3, respectively. The similarity is due to the fact that $\operatorname{Re}\left(\gamma^{S i}(h k l)\right)$ and $\operatorname{Im}\left(\gamma^{S i}(h k l)\right)$ represent the centrosymmetric and anisotropic components of the charge density distribution correction, respectively. Qualitative differences between 
the sets of plots are artifacts of the different plot ranges.

\section{Radial Dependence}

We will now inspect the radial dependence of $\operatorname{Im}\left(\gamma^{S i}(h k l)\right)$ along certain reciprocal lattice directions. Figure 5.6 shows calculated values of $\operatorname{Im}\left(\gamma^{S i}(h k l)\right)$, at 300K, along the $\langle 222\rangle,\langle 442\rangle$, and $\langle 622\rangle$ directions, where the $\left(h_{0}, k_{0}, l_{0}\right)=(222)$, (442), and (622) Si forbidden reflections are at $n=1$ and where $(h k l)=\left(h_{0}, k_{0}, l_{0}\right) n$. The horizontal axis is along the reciprocal lattice vector that goes between the origin and the forbidden reflection in reciprocal space. The horizontal scale of each curve has been adjusted so that the forbidden reflections line up at $n=1$, and so that the second and third multiples of the reflections occur at $n=2$ and $n=3$ in the plot, respectively. Negative $\operatorname{Im}\left(\gamma^{S i}(h k l)\right)$ values corresponds to charge anisotropy that is dominated by the bonding asymmetry, while positive values correspond to the anharmonic vibrations of the core. The dip appearing below $n=1$ is related to the bonding asymmetry, which is spread out in real-space and appears as a sharper feature in reciprocal space. Comparing the amplitude of the dips we can also discern that the bonding asymmetry is greater along $\langle 222\rangle$ than along the $\langle 622\rangle$ direction.

At higher $n$, the behavior of $\operatorname{Im}\left(\gamma^{S i}(h k l)\right)$ corresponds to charge anisotropy features that are dominated by anharmonic thermal vibrations.

It is worth mentioning again that at non-forbidden reflections the scattering contribution from the spherical charge density distribution effectively overwhelms the contributions from the anisotropic charge density distribution. Therefore, the only opportunities within the range shown in Fig. 5.6 to experimentally measure the charge anisotropy, $\operatorname{Im}\left(\gamma^{S i}(h k l)\right)$, alone are at $n=1$ and $n=3$. Furthermore, considering that the three lowest index forbidden reflections for Si are, in order, (222), (442), and (622) and that higher index reflections will be dominated by the anharmonic 

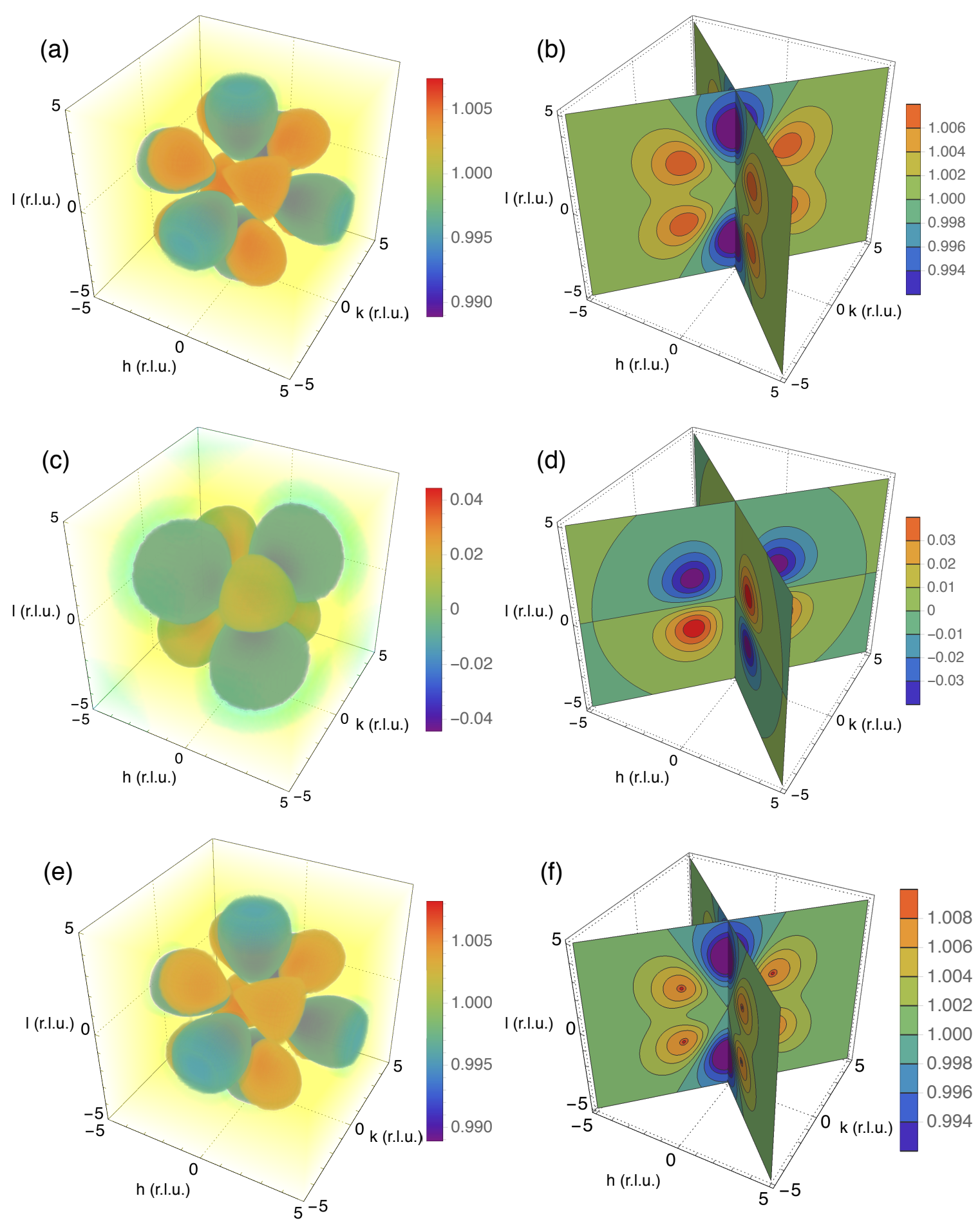

Figure 5.5: Three-dimensional plots of ((a) and (b)) $\operatorname{Re}\left(\gamma^{S i}(h k l)\right),((\mathrm{c})$ and (d)) $\operatorname{Im}\left(\gamma^{S i}(h k l)\right)$, and ((e) and (f)) $\left|\gamma^{S i}(h k l)\right|$. The left and right are three-dimensional density plots and complimentary cross-sectional contour plots, respectively. The cross-sectional planes correspond to (110) and (110). The plots are calculated for $300 \mathrm{~K}$. 


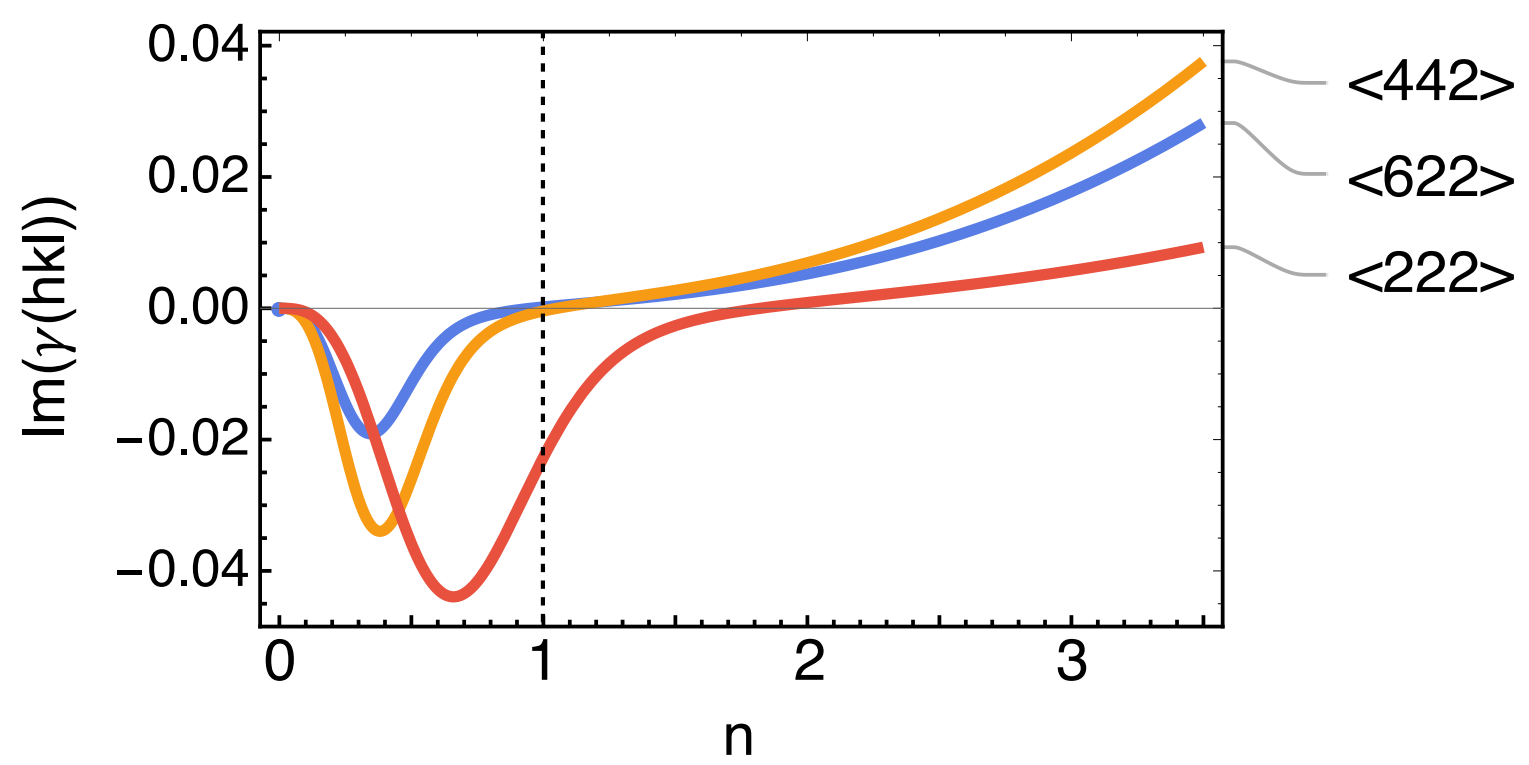

Figure 5.6: Calculated value of $\operatorname{Im}(\gamma(h k l))$ for $\mathrm{Si}$, at 300K, along the $\langle 222\rangle,\langle 442\rangle$, and $\langle 622\rangle$ directions. The horizontal axis is along the reciprocal lattice vector that starts at the origin and intersects with the (222), (442), and (622) forbidden reflections, respectively, at $n=1$. $n=2$ and $n=3$ correspond to the second and third multiples, respectively, of the forbidden reflections.

vibration component, the opportunity to experimentally measure charge anisotropy that is dominated by bonding asymmetry contributions is quite limited.

\section{Temperature Dependence}

The change in $\gamma^{S i}(h k l)$ between $100 \mathrm{~K}$ and $900 \mathrm{~K}$ is shown Fig. 5.7, where the difference is determined by

$$
\Delta \gamma^{S i}(h k l)=\gamma^{S i}(h k l ; 900 K)-\gamma^{S i}(h k l ; 100 K)
$$

The difference in the real part, $\operatorname{Re}\left(\Delta \gamma^{S i}(h k l)\right)$, is shown in Fig. 5.7(a) and (b), where eight egg-shaped lobes extend out from the origin to the corners of the cube and accounts for the redistribution of the centrosymmetric core charge. The impact of the anharmonic thermal vibrations is noticeable in Fig. 5.7(c) and (d), which show the 
change in the imaginary part, $\operatorname{Im}\left(\Delta \gamma^{S i}(h k l)\right) \cdot \operatorname{Im}\left(\Delta \gamma^{S i}(h k l)\right)$ is concentrated at the corners of the cube due to the anharmonic thermal vibrations, which redistribute the charge in the direction opposite of the bonds. The lobe-like features previously seen in Fig. 5.5(c) and (d) are absent in Fig. 5.7(c) and (d). The absence of these features is because the asymmetric bonds have a relatively minor temperature dependence. Lastly, the similarity between $\operatorname{Im}\left(\Delta \gamma^{S i}(h k l)\right)$ and $\left|\Delta \gamma^{S i}(h k l)\right|$ (shown in Fig. 5.7(e) and (f)) indicates that the temperature dependence of the imaginary part is significantly greater than the real part. Or in other words, the temperature dependence of the anisotropic charge density distribution is greater than the centrosymmetric charge density distribution.

Figure 5.8 shows the calculated temperature dependence of $\operatorname{Im}\left(\gamma^{S i}(h k l)\right)$ for the (222), (442), and (622) Si forbidden reflections. The calculation for this plot used previously determined Si parameters for the charge density distribution[59] and the anharmonic temperature[68]. $\operatorname{Im}(\gamma)$ is negative below $\sim 200 \mathrm{~K}$, for all three forbidden reflections, which corresponds to the asymmetric bonding dominating the charge anisotropy. At higher temperature, $\operatorname{Im}\left(\gamma^{S i}(442)\right)$ and $\operatorname{Im}\left(\gamma^{S i}(622)\right)$ are both positive, which indicates that the anharmonic thermal vibrations are dominating the charge anisotropy. The difference in the slope of the three curves reflects the varying influence of the anharmonic thermal vibrations at the different forbidden reflections. The relatively minor influence of the anharmonic thermal vibrations on the 222 reflection is illustrated by its shallower slope when compared to the other curves.

Figure 5.9 shows the calculated temperature dependence of $\operatorname{Im}\left(\gamma^{S i}(442)\right)$ and $\operatorname{Im}\left(\gamma^{S i}(44 \overline{2})\right)$. At low temperature $\operatorname{Im}\left(\gamma^{S i}(442)\right)$ is negative, which corresponds to the asymmetric bonding dominating the charge anisotropy. Then above $\sim 400 \mathrm{~K}$, $\operatorname{Im}\left(\gamma^{S i}(442)\right)$ is positive, which indicates a switch to the anharmonic thermal vibrations dominating the charge anisotropy. The switchover was first observed experimen- 

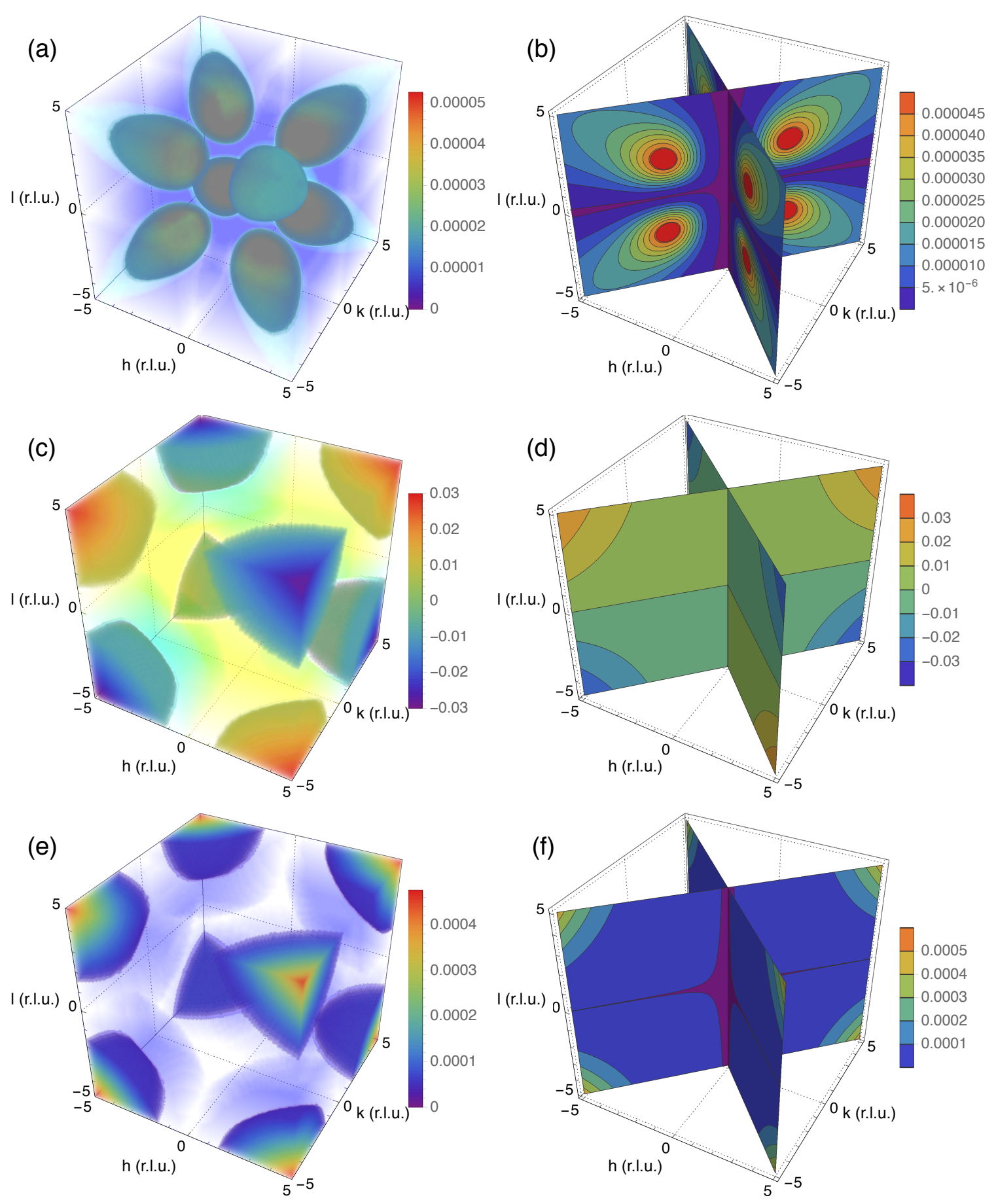

Figure 5.7: $\Delta \gamma^{S i}(h k l)$ between $100 \mathrm{~K}$ and 900K. Three-dimensional plots of ((a) and (b)) $\operatorname{Re}\left(\Delta \gamma^{S i}(h k l)\right),\left((\mathrm{c})\right.$ and (d)) $\operatorname{Im}\left(\Delta \gamma^{S i}(h k l)\right)$, ((e) and (f)) $\left|\Delta \gamma^{S i}(h k l)\right|$. The plots on the left are three-dimensional density plots and complimentary cross-sectional contour plots are on the right. The intersecting cross-sectional planes correspond to (110) and (110). 


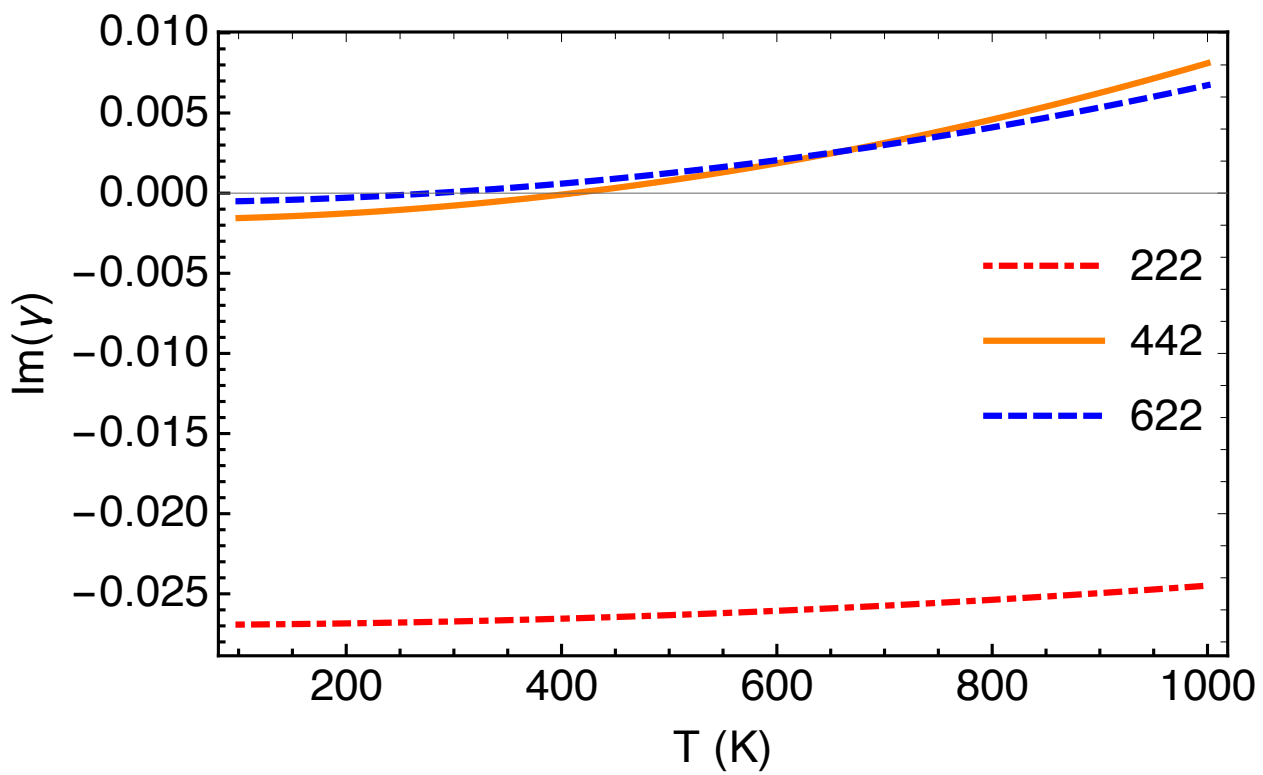

Figure 5.8: Calculated temperature dependence of $\operatorname{Im}\left(\gamma^{S i}(222)\right), \operatorname{Im}\left(\gamma^{S i}(442)\right)$, and $\operatorname{Im}\left(\gamma^{S i}(622)\right)$. The calculated curves uses Fehlmann's[59] values for $\alpha, K_{3,22}$, and $L_{3,22}$.

tally at $\sim 500 \mathrm{~K}$ by Trucano and Batterman[15] and is discussed further in Section 6.3. The discrepancy between the calculated crossover temperature shown in Fig. 5.9 and with Trucano is attributed to the charge density distribution parameters that were used in our calculation. Our calculations used previously determined[59] values for $\alpha$ and $K_{3,22}$ and $L_{4,22}$. A higher crossover temperature can be explained with a greater $\alpha$ value.

The change in sign between $\operatorname{Im}\left(\gamma^{S i}(442)\right)$ and $\operatorname{Im}\left(\gamma^{S i}(44 \overline{2})\right)$ is due to the change in direction of the scattering vector. When an odd number of Miller indices are negative the correction factor will experience a sign change, or in other words:

$$
\operatorname{Im}(\gamma(-\vec{Q}))=-\operatorname{Im}(\gamma(\vec{Q}))
$$

This behavior is expected because of the directional dependence of the anisotropic charge density distribution. As a result, a positive $\operatorname{Im}(\gamma(-\vec{Q}))$ value corresponds 


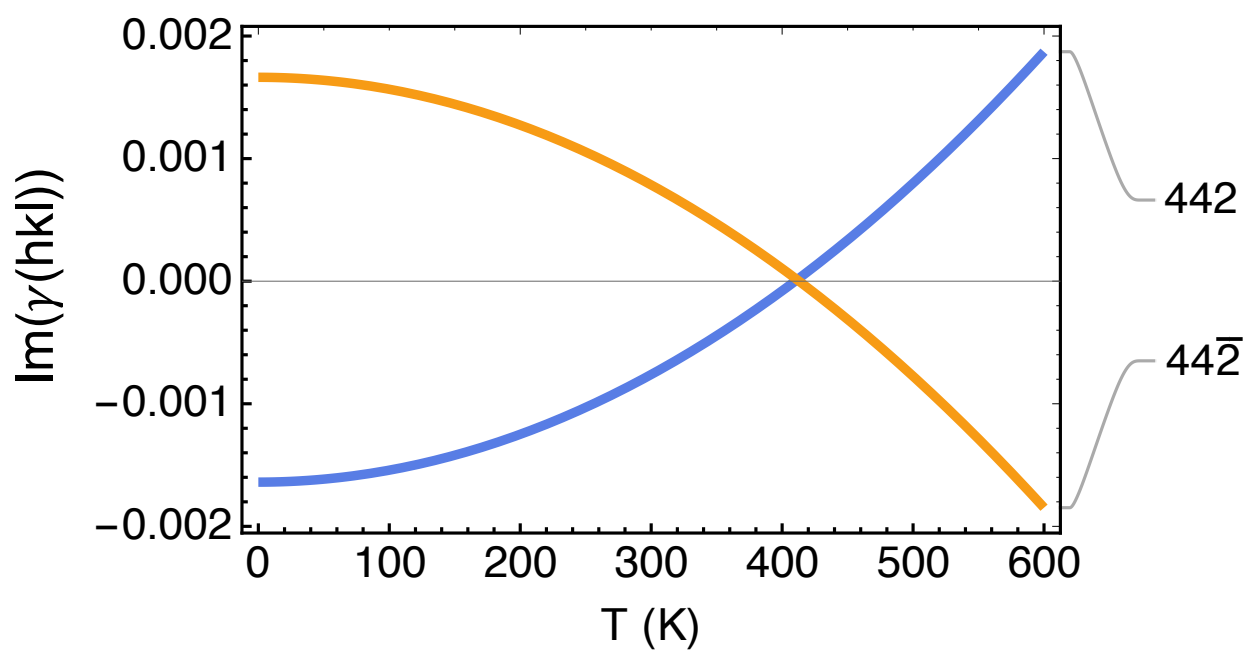

Figure 5.9: Calculated temperature dependence of $\operatorname{Im}\left(\gamma^{S i}(442)\right)$ and $\operatorname{Im}\left(\gamma^{S i}(44 \overline{2})\right)$. The calculated curves uses Fehlmann's[59] values for $\alpha, K_{3,22}$, and $L_{3,22}$.

to the case when bonding asymmetry dominates the net charge anisotropy, while a negative value indicates that the anharmonic vibrations are the dominant component.

\section{$\operatorname{Im}(\gamma(\mathbf{h k l}))$ of Select Si Forbidden Reflections}

A useful way to survey the anisotropic charge density distribution at different forbidden reflections is with three-dimensional bubble plots, which are shown in Fig. 5.10. The diameter of the spheres are proportional to $\left|\operatorname{Im}\left(\gamma^{S i}(\vec{Q})\right)\right|$ and the color indicates the dominant component of the charge anisotropy. At 100K, (Fig. 5.10(a)) the two largest spheres, shown in yellow, correspond to the charge anisotropy at the (222) and $(2 \overline{2} 2)$ forbidden reflections of $\mathrm{Si}$, which is dominated by the bonding asymmetry. Immediately beyond the $\operatorname{Si}(222)$ forbidden reflections are the (442) and (622) sets of reflections. These reflections exhibit a notably smaller net charge anisotropy than (222), which is dominated by a significantly smaller contribution from the asymmetric bonding and this fact was also seen in Fig. 5.6. Correspondingly, the small yellow spheres for the (442) and (622) forbidden reflections are too small to see in this plot 
without magnification. The remaining spheres are colored in cyan and correspond to higher index forbidden reflections, which are dominated by anharmonic thermal vibrations. The dramatic small size of the spheres at the higher index reflections indicates that at $100 \mathrm{~K}$ the net anisotropic charge at these reflections is significantly smaller than at the $\operatorname{Si}(222)$ reflection.

At a low temperature of $100 \mathrm{~K}$, one may expect the contribution from the anharmonic thermal vibrations to be greatly diminished; however, the anisotropic charge density distribution at the higher index reflections remains dominated by the anharmonic thermal component and not by the bonding asymmetry. This behavior is due to two factors: First, the contribution from the bonding asymmetry will be the greatest at low $\vec{Q}$ and then it diminish at higher $\vec{Q}$. This behavior was also seen in Fig. 5.6. Secondly, the anharmonic contribution increases with the triple product of the Miller indices, as seen by Eq. (5.19), therefore the anharmonic contributions will be greater at higher $\vec{Q}$.

The temperature and $h k l$ dependence of the charge anisotropy is more apparent in Fig. 5.10(b) and (c), where the temperature is at $300 \mathrm{~K}$ and $400 \mathrm{~K}$, respectively. The $\operatorname{Si}(222)$ spheres appear to remain the same size throughout the temperature range shown. Whereas the anharmonic thermal vibrations, indicated by the cyan spheres, grow with the square of the temperature. This behavior is expected because the anharmonic thermal contribution to the charge anisotropy at the (222) reflection is negligible, while at higher index reflections the anharmonic contributions tend to dominate with elevated temperature, as indicated by Eq. (5.19). Furthermore, the cyan spheres appear to get larger further from the origin, which reflects the $h k l$ dependence of the anharmonic thermal vibrations.

The three-dimensional bubble plot in Fig. 5.11 is a useful way to survey sign change of $\operatorname{Im}\left(\gamma^{S i}(\vec{Q})\right)$. Here the colors of the spheres indicate the sign of the anisotropy 

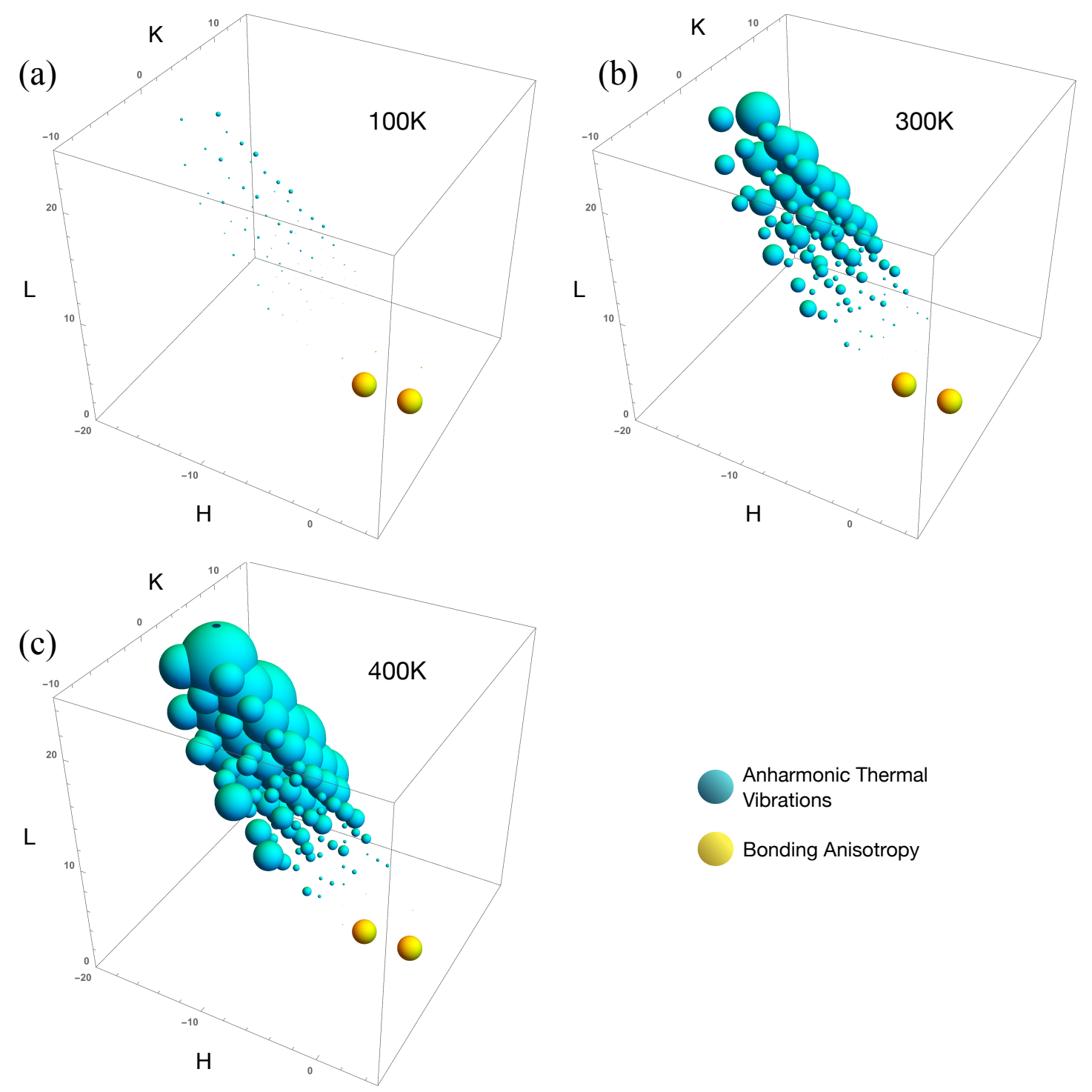

Anharmonic Thermal

Vibrations

Bonding Anisotropy

Figure 5.10: Three dimensional bubble plots of $\operatorname{Im}\left(\gamma^{S i}(h k l)\right)$ for select forbidden reflections at (a) $100 \mathrm{~K}$, (b) $300 \mathrm{~K}$, and (c) 400K. The diameter of the spheres are proportional to the magnitude of $\operatorname{Im}(\gamma(\operatorname{Si}(h+k+l=4 n+2)))$. The color indicates whether the asymmetric bonding (yellow) or anharmonic thermal vibrations (cyan) are the dominant contribution to the charge anisotropy. 
correction factor rather than the dominant contribution of the charge anisotropy. Positive and negative values of $\operatorname{Im}\left(\gamma^{S i}(\vec{Q})\right)$ are represented with cyan and yellow spheres, respectively. It can be seen that $\operatorname{Im}\left(\gamma^{S i}(\vec{Q})\right)$ experiences a sign change when crossing a reciprocal axis. For example, the $\left.\operatorname{Im}\left(\gamma^{S i}(222)\right)\right)$ is shown in yellow near the origin, whereas the $\left.\operatorname{Im}\left(\gamma^{S i}(2 \overline{2} 2)\right)\right)$ is positive and shown in cyan, even though both are dominated by the bonding asymmetry. Furthermore, it can also be seen that when an odd number of Miller indices are positive the original sign convention can be used.

\subsection{Hexagonal Coordinates}

It is convenient to use hexagonal coordinates when examining a (111) terminated FCC or the diamond crystal structure. We will use the FCC lattice to establish our hexagonal coordinate system and subscripts ' $C$ ' and ' $H$ ' will be used to distinguish between cubic and hexagonal coordinates. The $(111)_{C}$ FCC unit cell contains three layers of $(111)_{C}$ FCC planes with an atom located on each plane. Figure 5.12 shows an example of a $(111)_{C}$ FCC plane in a cubic FCC lattice and a cross-sectional view of the FCC unit cell. The distance between the nearest neighbors atoms in the $(111)_{C}$ plane is $a_{H}=\frac{a_{C}}{\sqrt{2}}$, where $a_{C}$ is the cubic FCC lattice constant and $a_{H}$ is the hexagonal lattice constant. The distance between the $(111)_{C}$ planes is $c_{H}=\sqrt{3} a_{C} \cdot \vec{a}_{1}$ and $\vec{a}_{2}$ are the in-plane lattice vectors, which are $120^{\circ}$ apart from one another, and $\vec{a}_{3}$ is the out-of-plane lattice vector. The relationship between the real-space primitive lattice vectors, in hexagonal coordinates, and Cartesian coordinates, in terms of the cubic lattice constant $a_{C}$, are[72]

$$
\vec{a}_{1}=\frac{a_{C}}{\sqrt{2}} \hat{x} ; \quad \vec{a}_{2}=\frac{a_{C}}{\sqrt{2}}\left(\frac{-1}{2} \hat{x}+\frac{\sqrt{3}}{2} \hat{y}\right) ; \vec{a}_{3}=\sqrt{3} a_{C} \hat{z}
$$




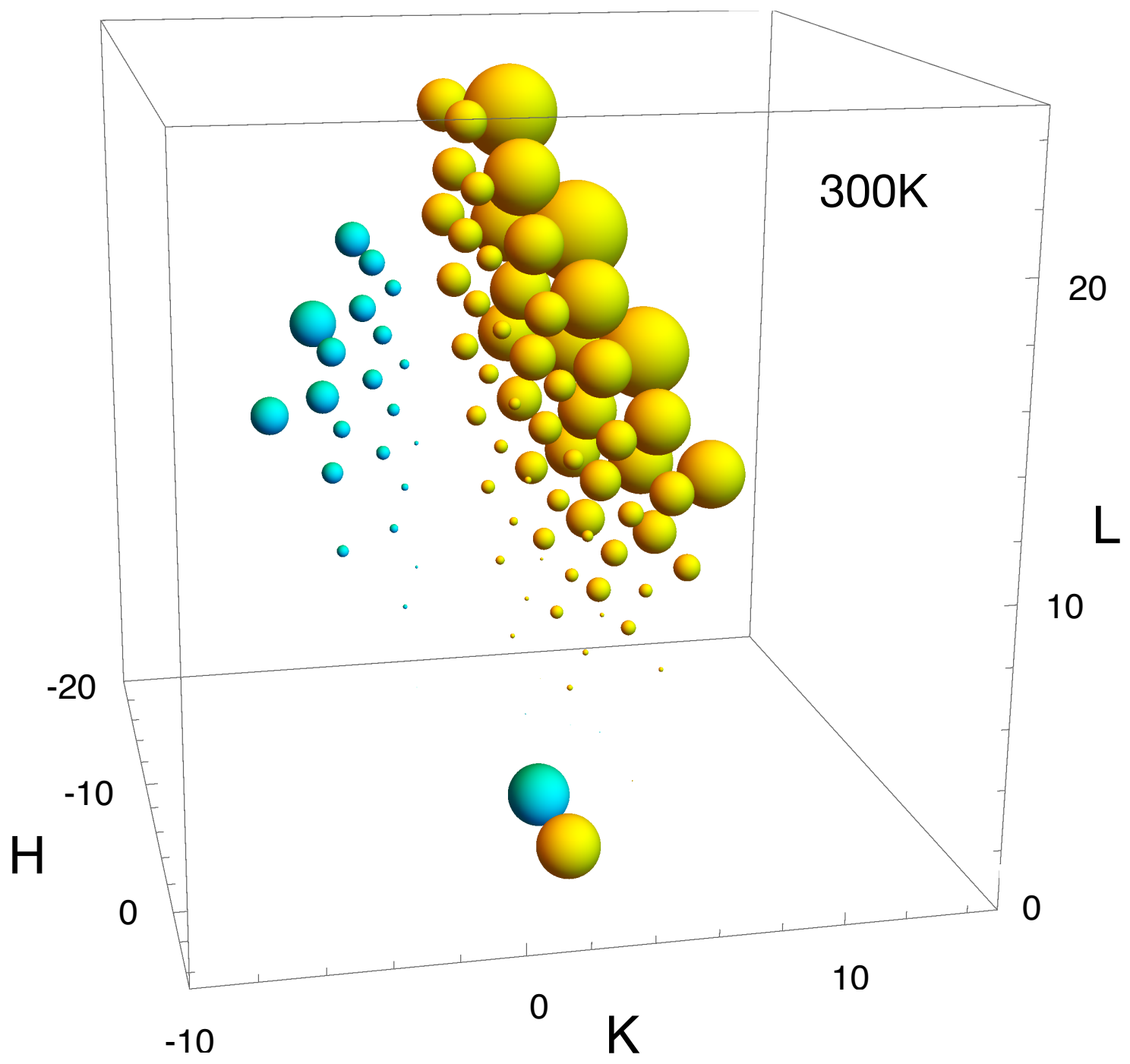

\section{Positive $\operatorname{Im}(\gamma(\mathrm{hkl}))$}

\section{Negative $\operatorname{Im}(\gamma(\mathrm{hkl}))$}

Figure 5.11: Three dimensional bubble plot showing the sign of $\operatorname{Im}\left(\gamma^{S i}(h k l)\right)$ for select forbidden reflections at $300 \mathrm{~K}$. The diameter of the spheres are proportional to the magnitude of $\operatorname{Im}\left(\gamma^{S i}(h k l)\right)$. The color indicates the $\operatorname{sign}$ of $\operatorname{Im}\left(\gamma^{S i}(h k l)\right)$ : positive and negative values are represented by cyan and yellow spheres, respectively. 
and in terms of the hexagonal lattice constant $a_{H}$ are

$$
\vec{a}_{1}=a_{H} \hat{x} ; \quad \vec{a}_{2}=a_{H}\left(\frac{-1}{2} \hat{x}+\frac{\sqrt{3}}{2} \hat{y}\right) ; \vec{a}_{3}=c_{H} \hat{z}
$$

\section{Relationship of Hexagonal and Cubic Coordinates}

The hexagonal and cubic Miller indices are related by

$$
\begin{aligned}
& H_{H}=\frac{1}{2}\left(-H_{C}+K_{C}\right) ; \\
& K_{H}=\frac{1}{2}\left(-K_{C}+L_{C}\right) ; \\
& L_{H}=H_{C}+K_{C}+L_{C}
\end{aligned}
$$

and

$$
\begin{aligned}
& H_{C}=\frac{1}{3}\left(-4 H_{H}-2 K_{H}+L_{H}\right) ; \\
& K_{C}=\frac{1}{3}\left(2 H_{H}-2 K_{H}+L_{H}\right) ; \\
& L_{C}=\frac{1}{3}\left(2 H_{H}+4 K_{H}+L_{H}\right) .
\end{aligned}
$$

\section{FCC $(111)_{C}$ Structure Factor in Hexagonal Coordinates}

Each $(111)_{C}$ layer in the $\mathrm{FCC}(111)_{C}$ unit cell is translated by

$$
\vec{r}_{1}=\frac{2}{3} \vec{a}_{1}+\frac{1}{3} \vec{a}_{2}+\frac{1}{3} \vec{a}_{3}
$$

Therefore the structure factor for the $\mathrm{FCC}(111)_{C}$ unit cell in hexagonal coordinates is expressed as

$$
F_{F C C(111)_{C}}=1+e^{-i \vec{Q} \cdot \vec{r}_{1}}+e^{-i \vec{Q} \cdot 2 \vec{r}_{1}}
$$



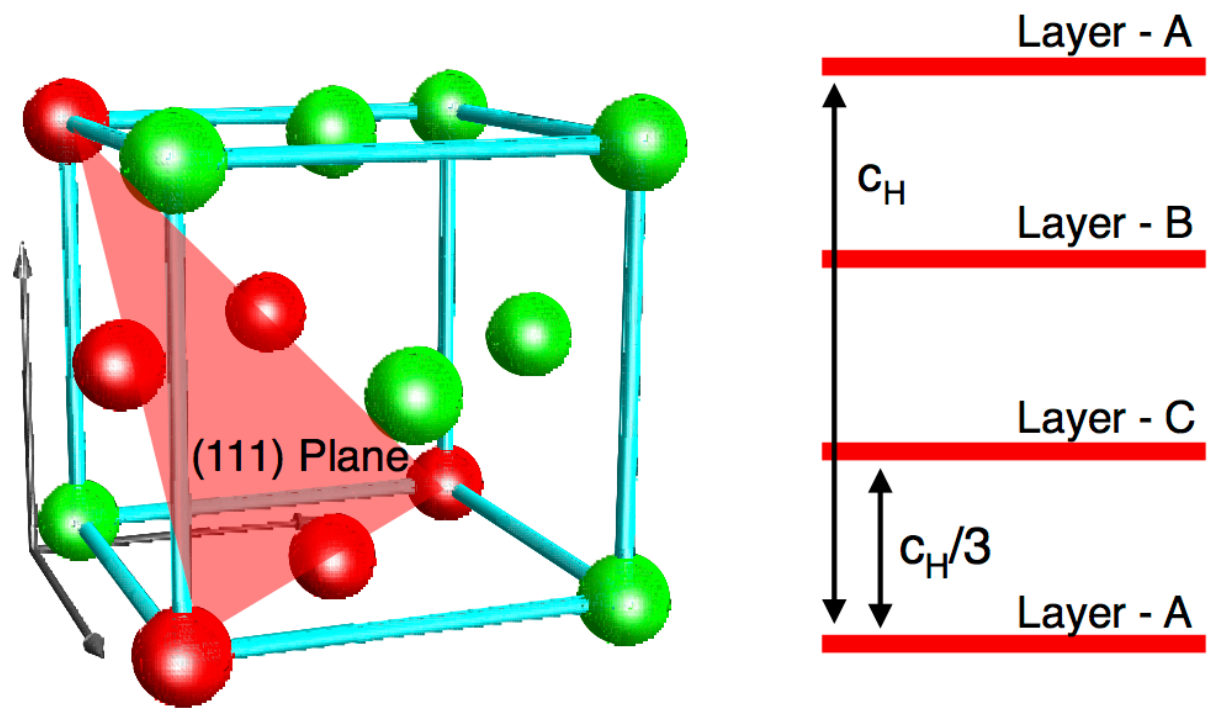

Figure 5.12: On the left is a schematic of the $(111)_{C}$ plane in a FCC lattice. The diagram on the right shows the A-B-C stacking of $\mathrm{FCC}(111)_{C}$ planes. The lattice constant in hexagonal coordinates is $c_{H}$ and the layer spacing is $\frac{c_{H}}{3}$.

where $\vec{Q} \cdot \vec{r}_{1}=2 \pi\left(\frac{2}{3} H_{H}+\frac{1}{3} K_{H}+\frac{1}{3} L_{H}\right)$ and $H_{H}, K_{H}, L_{H}$ are the Miller indices in hexagonal coordinates. Note that Eq. (5.39) is not the same as the FCC structure factor in cubic coordinates, Eq. (5.22).

\section{Diamond Crystal Structure in Hexagonal Coordinates}

Now lets consider the structure factor in hexagonal coordinates for the diamond crystal structure. Figure 5.13 shows a cross-sectional and top view of the $(111)_{C}$ diamond crystal structure. The basis sites of the two interpenetrating FCC lattices are distinguished by different colors. The atomic layers of the two basis sites exhibit A-B stacking where the B-site is translated from the A-site by $\vec{r}_{A}=\frac{2}{3} \vec{a}_{1}+\frac{1}{3} \vec{a}_{2}+\frac{1}{12} \vec{a}_{3}$. The DL unit cell structure factor in hexagonal coordinates is

$$
\begin{aligned}
F_{D L} & =f^{A}(\vec{Q})+f^{B}(\vec{Q}) e^{-i \vec{Q} \cdot \vec{r}_{A}} \\
& =f^{A}(\vec{Q})+f^{B}(\vec{Q}) e^{-i 2 \pi\left(\frac{2}{3} H_{H}+\frac{1}{3} K_{H}+\frac{1}{12} L_{H}\right)} .
\end{aligned}
$$




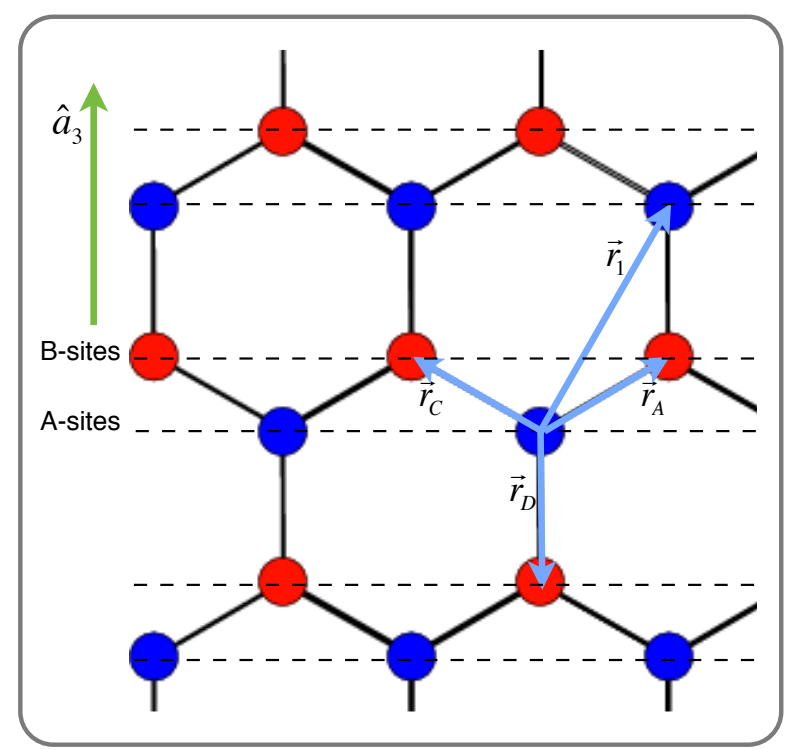

Side View

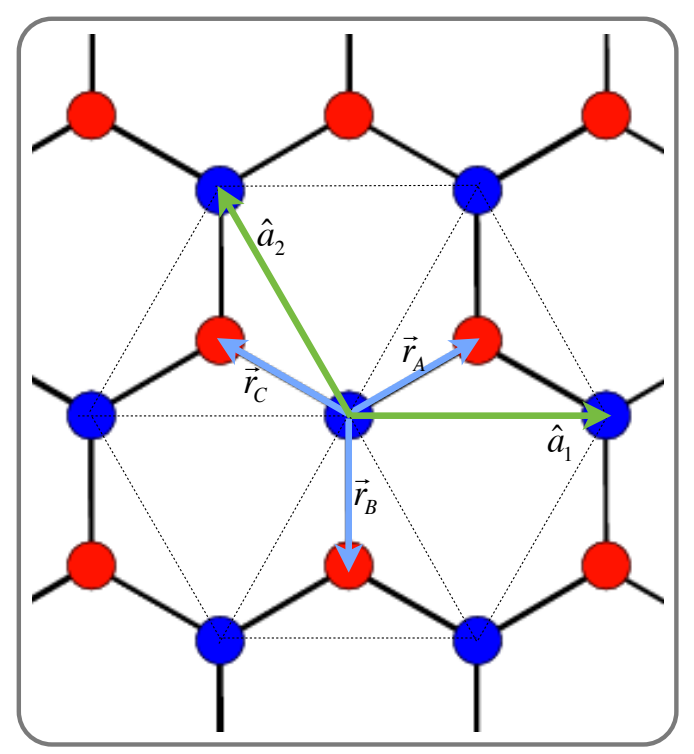

Top View

Figure 5.13: The left panel is a profile view of the diamond-structure $(111)_{C}$ planes in real-space. The vertical direction is along $\langle 111\rangle_{C}$. The right panel shows the top view of the $(111)_{C}$ planes of the diamond-structure lattice in real-space. The A and B sites are color coded blue and red, respectively. $\hat{a}_{n}$ are the unit vectors in hexagonal coordinates. The in-plane vectors, $\hat{a}_{1}$ and $\hat{a}_{2}$, are 120 degrees apart and $\hat{a}_{3}$ is the out of plane vector. $\vec{r}_{j}$ are the lattice vectors.

The product of the FCC $(111)_{C}$ and DL structure factors, Eq. (5.39) and Eq. (5.40), is the structure factor for the diamond-structure in hexagonal coordinates:

$$
\begin{aligned}
F_{\text {Dia }}= & F_{F C C(111)_{C}} F_{D L} \\
= & {\left[1+e^{-i \vec{Q} \cdot \vec{r}_{1}}+e^{-i \vec{Q} \cdot 2 \vec{r}_{1}}\right]\left[f^{A}(\vec{Q})+f^{B}(\vec{Q}) e^{-i \vec{Q} \cdot \vec{r}_{A}}\right] } \\
= & {\left[1+e^{-i 2 \pi\left(\frac{2}{3} H_{H}+\frac{1}{3} K_{H}+\frac{1}{3} L_{H}\right)}+e^{-i 4 \pi\left(\frac{2}{3} H_{H}+\frac{1}{3} K_{H}+\frac{1}{3} L_{H}\right)}\right] } \\
& {\left[f^{A}(\vec{Q})+f^{B}(\vec{Q}) e^{-i 2 \pi\left(\frac{2}{3} H_{H}+\frac{1}{3} K_{H}+\frac{1}{12} L_{H}\right)}\right] . }
\end{aligned}
$$




\section{Chapter 6}

\section{Background and Previous Work}

\subsection{Introduction}

Determining charge densities experimentally and theoretically has been of great interest for the past $40+$ years,[73] with a significant amount of activity taking place during the 70's. [50] A notable example is the experimental work conducted by Batterman et al., starting in 1970 Batterman and colleagues[13] performed a series of temperature dependent X-ray and complimentary neutron diffraction studies on forbidden Bragg reflections from Si and Ge over the course of two decades. The combination of X-rays and neutrons allowed them to deconvolute the density distributions of the valence electrons from the core, because the former experimental tool is sensitive to both the core and valence electrons, whereas neutrons are sensitive to the nucleus of an atom. Studying these forbidden Bragg reflections were critically important for determining the fine details of the structure of the charge density distribution because their diffracted intensity is entirely dependent on electron bonding and anharmonic effects.[57] By comparison, in conventionally allowed Bragg reflections, these small 
effects are overwhelmed by the scattering contribution from the significantly larger spherical charge density distribution. These studies along with many others have improved our understanding of the electronic structures that dictate many characteristics of matter. $[49,51]$

Interestingly, recent advances in computing power, improved experimental instruments and hardware, and brighter X-ray sources have allowed for advances in experimentally determined charge densities - which has catalyzed a renewed interest on the topic. $[50,74,75]$ As a consequence, the results from modern experiments are able to challenge conventional theoretical models and shed light on their deficiencies.[50, 76] For example, in a study published in 2014, Bindzus et al.[73] experimentally determined that the conventional method of treating the core electron distribution as an inert sphere was insufficient to explain their highly accurate synchrotron X-ray diffraction data. Their results revealed finer features of the electron density distribution, which included deformation of the core electron density distribution. Considering that over the past few years new results have challenged convention and that only a minor fraction of the published literature on charge density is based on synchrotron scattering experiments, [74] the opportunity for further advances in the field is promising $[50]$.

In this chapter, we will review previous work on experimentally determined charge density distribution. In particular, we review several studies that utilized forbidden Bragg reflections to investigate the fine details of charge density distribution in diamond crystal structures, like $\mathrm{Si}$ and Ge. In Section 6.2 we will briefly review the formalism of determining charge density distribution from X-ray scattering, which compliments the discussions on charge density distribution in real-space and reciprocal-space found in Chapters 4 and 5, respectively. Section 6.3 will review the work of Batterman et al., who over the course of several decades of experimental work 
made significant early contributions to the study of charge anisotropy in $\mathrm{Si}$ and Ge. Lastly, Section 6.4 reviews examples of recent studies.

\subsection{Electron Density Distribution from X-ray Scat- tering}

As we discussed above, the observed X-ray intensity, $I(\vec{Q})$, is proportional to the modulus square of the scattering amplitude, see Eq. (5.20). Keeping in mind that the amplitude of the X-rays scattering from atoms in a crystalline structure can be determined by the structure factor of the material, $F(\vec{Q})$. The atomic charge density distribution can therefore be determined from X-ray measurements by taking the inverse Fourier transform of the structure factor and it is expressed here in integral form as well as an infinite sum of the structure factors[51]

$$
\rho(\vec{r})=\int F(\vec{Q}) e^{i \vec{Q} \cdot \vec{r}} d^{3} r=\frac{1}{V} \sum_{\vec{Q}} F(\vec{Q}) e^{i \vec{Q} \cdot \vec{r}}
$$

where $V$ is the unit cell volume.

\subsection{Early Forbidden Bragg Reflection Studies by Batterman et al. and Related Work}

The first observation of a forbidden X-ray reflection was by W. H. Bragg in 1920 when he measured the $(222)_{C}$ reflection from carbon diamond.[70] Considering spherical charge density distribution for each atom, the calculated structure factor for the $(222)_{C}$ reflection from a diamond structure lattice is zero, because the scattering is expected to deconstructivly interfere and extinguish. As we saw from Eq. (5.26), this 
reflection as well as others with Miller indices that satisfy $\left(h_{C}+k_{C}+l_{C}\right)=4 n+2$ are considered forbidden for diamond crystal structures. Bragg explained that the presence of the forbidden reflection invalidates the hypothesis that there is an isotropic force around an atom. Therefore, considering that a spherical charge does not contribute to a forbidden reflection, the observed scattering is entirely (neglecting sources of multiple scattering events) from the part of the charge density distribution that is anisotropic.

50 years after W. H. Bragg[70] first observed a forbidden X-ray reflection, in 1970 Batterman et al.[13] started on a series of temperature dependent X-ray and complimentary neutron diffraction measurements of forbidden Bragg reflections from Si and Ge. Over the course of two decades Batterman and colleagues experimentally investigated fine features in the charge density distribution including the magnitude, direction, and the components of the charge anisotropy in Si and Ge.

These studies took advantage of the fact that X-rays and neutrons are sensitive to matter in different ways, in order to study the components of the anisotropic charge density distribution. X-rays probe the atomic electron density, which includes both the core and valence electrons. Whereas neutrons are sensitive to the atomic nucleus. Therefore a combination of X-ray and neutron diffraction measurements would be able to parse out structural information of the covalent bonds from the X-ray data. Furthermore, the combination of the two techniques were required to deconvolute the contributions because phase information is lost in diffraction measurements.

In an early study,[13] the temperature dependence of the X-ray $\operatorname{Si}(222)_{C}$ forbidden Bragg reflection was studied by measuring the integrated intensity of the $(222)_{C}$ peak relative to the conventionally allowed $(111)_{C}$ and $(333)_{C}$ peaks. The $\mathrm{Si}(222)_{C}$ integrated intensity was observed to decrease as the temperature increased, which suggests that the $\mathrm{Si}(222)_{C}$ forbidden reflection is primarily due to bonding effects 
rather than anharmonic thermal vibrations of the core - as seen in Eq. (5.19), anharmonic thermal vibrations are expected to increase the overall anisotropic charge density distribution with higher temperature rather than decrease it. Two different models were proposed to explain the $\mathrm{Si}(222)_{C}$ temperature dependence:[13]

1. The temperature dependence is due to bonding electrons that vibrate with the core electrons, which assumes that the Debye-Waller factors of the core and the bonding electrons are the same. This model is often referred to as the "ridged-ion model."

2. The bonding electrons do not vibrate and that the temperature dependence is due to the anharmonic thermal motion of the core, which assumes different Debye-Waller factors for the core and bonding electrons.

Due to experimental uncertainties in this study, the origin of the $\mathrm{Si}(222)_{C}$ temperature dependence remained inconclusive.

What is the origin of the Si(222) $C$ temperature dependence? Succeeding studies sought to answer this question. The results of a $\operatorname{Si}(222)_{C}$ X-ray diffraction study by Keating et al.[14] indicated that the bonding electrons vibrate with half the mean square amplitude of the core, which suggests the failure of the ridged-ion model. Meanwhile temperature dependent neutron diffraction measurements of $\mathrm{Si}(222)_{C}$ confirmed that the thermal vibrations of the core are in fact anharmonic.[77] In contrast to the results of Keating et al.[14], subsequent temperature and pressure dependent Xray diffraction measurements of the $\mathrm{Si}(222)_{C}$ reflection by Fujimoto[78] indicated that the thermal vibration mean-square amplitudes of the bonds and core are equivalent within experimental uncertainty, which supports the ridged-ion model. Fujimoto's results were then later corroborated by a neutron and X-ray diffraction study of $\mathrm{Si}(222)_{C}$ and $\mathrm{Ge}(222)_{C}$ by Roberto et al.[66] Roberto's work also improved upon the 
group's previous studies[14, 77], which resulted in a revised conclusion of the vibrational behavior of the bonds and core, which now agrees with the ridged-ion model. Furthermore, the results by Roberto et al. also indicated that the anharmonic thermal behavior of Si and Ge are generally consistent with Eq. (5.13), Dawson's[62] single-atom Einstein potential. The conflicting results reflect the difficulty of these experiments.

What is the temperature dependence of higher index forbidden Bragg reflections? A study by Truncano and Batterman[15] sought to further understand the bonding electron and anharmonic charge density distribution of Si by examining the temperature dependence of the $\mathrm{Si}(442)_{C}$ X-ray forbidden reflection. The measured integrated intensity exhibited interesting temperature behavior, as seen in Fig. 6.1, which was a departure from the temperature dependence previously observed in $\operatorname{Si}(222)_{C}$ (where the intensity is inversely dependent on the temperature). It can be seen that at low temperature the integrated intensity decreases with increasing temperature, reaching zero around $\sim 500 \mathrm{~K}$, and then increases at higher temperatures. This result elegantly demonstrated that two competing contributions to the charge anisotropy act in opposite directions.

The competing contributions are illustrated schematically in Fig. 6.2. The bonding electrons are directed to the nearest neighbor atoms, as seen in Fig. 6.2(a). Whereas the core thermally vibrate anharmonically towards the voids opposite the bonds, as seen in Fig. 6.2(b). Thus at low temperature, when the amplitude of the thermal vibrations are small, the net charge anisotropy would be dominated by the contributions from the bonds. As the temperature increases so will the anharmonic vibrations and the net charge anisotropy will decrease as the core vibrates further away from the bonds and toward the void. Around $500 \mathrm{~K}$ the contributions are equivalent and cancel out. At higher temperature the net charge anisotropy will be dominated by 


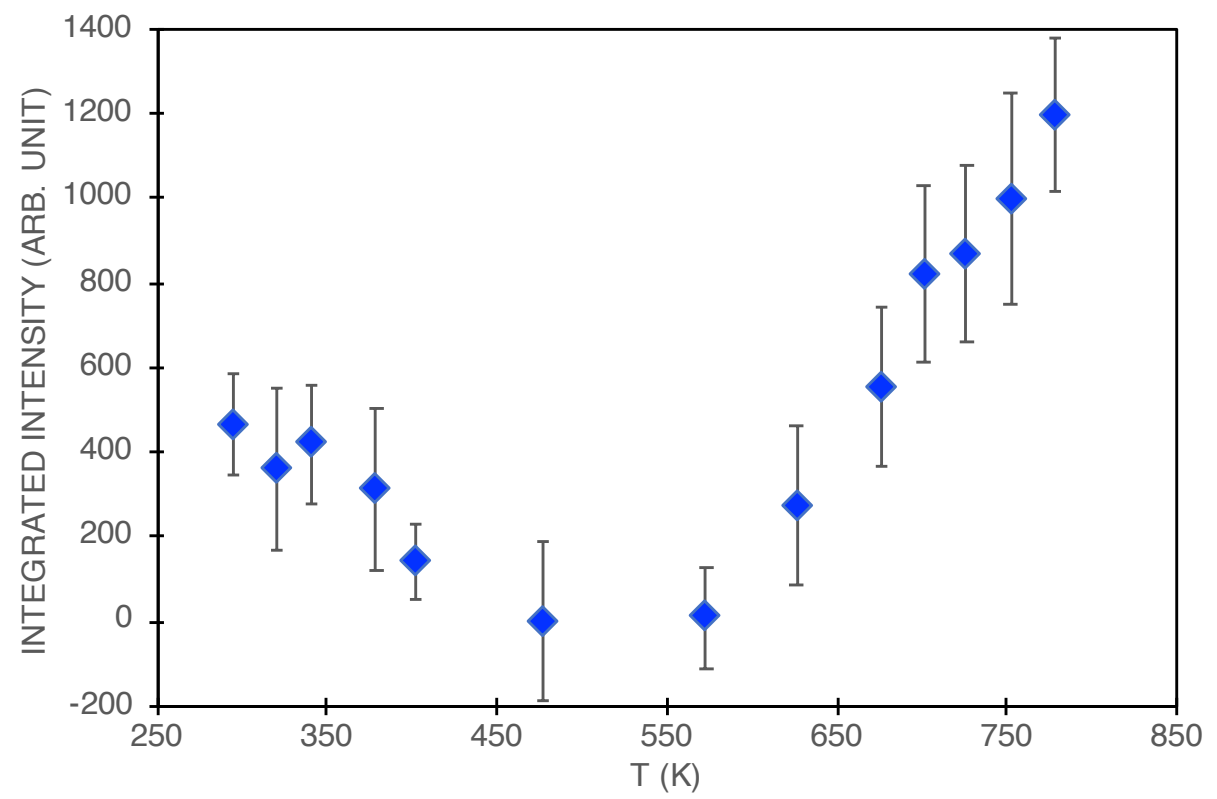

Figure 6.1: Temperature dependence of the $\mathrm{Si}(442)_{C}$ integrated X-ray intensity. The plot is reproduced using data from Trucano and Batterman[15].

the anharmonic core contribution.

As a thought exercise, lets consider a hypothetical situation where the charge anisotropy contributions had the same sign. Or in other words the contributions would act in the same direction (rather than in opposite directions illustrated in Fig. 6.2). In this situation, the $\operatorname{Si}(442)_{C}$ intensity would only increase with temperature and therefore not agree with the behavior seen in Fig. 6.1. Only a scenario where the anisotropic charge density distribution contributions compete can explain the temperature dependence observed in Fig. 6.1.

Truncano and Batterman[15] expressed the two competing contributions of the forbidden reflection with the following X-ray structure factor

$$
\begin{aligned}
F(h+k+l=4 n+2) & =-F_{\text {bond }}+F_{\text {core }} \\
& =8 i\left(-f_{\text {bond }} e^{-M_{\text {bond }}}+f_{\text {core }} e^{-M_{\text {core }}} A(h k l ; T)\right),
\end{aligned}
$$


(a) Bonding Directions

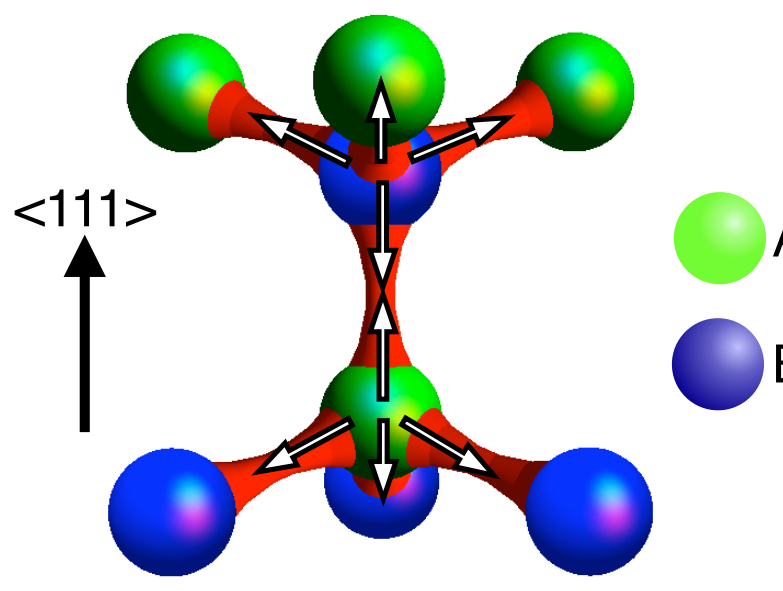

(b) Anharmonic Thermal

A-Site

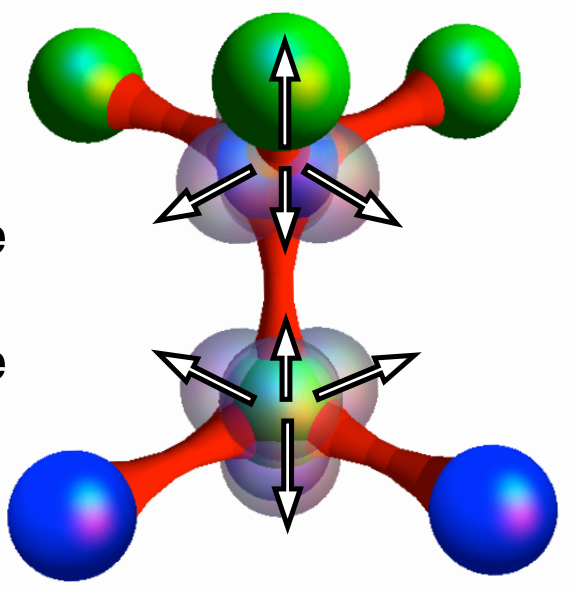

Figure 6.2: Schematic diagram of the tetrahedral configuration of the A-B sites and their nearest neighbors in diamond-structure crystals (a) The arrows point in the bonding directions toward the nearest neighbor atoms. (b) Shows the directions of the anharmonic thermal vibrations.

where $F_{\text {bond }}$ and $F_{\text {core }}$ are the structure factors, $f_{\text {bond }}$ and $f_{\text {core }}$ are the atomic form factors, $e^{-M_{\text {bond }}}$ and $e^{-M_{\text {core }}}$ are the Debye-Waller factors of the bonds and core, respectively. $A(h k l ; T)$ is the anharmonic vibrational factor and is determined by[77]

$$
A(h k l ; T)=\left(\frac{2 \pi}{\alpha_{t}}\right)^{3} \frac{\beta_{t}}{V} h k l\left(k_{B} T\right)^{2}
$$

where $\alpha_{t}$ and $\beta_{t}$ are the harmonic and anharmonic force constants, respectively, from the single-atom potential seen in Eq. (5.13), $V$ is the volume of the unit cell, $h k l$ are the Miller indices, $k_{B}$ is the Boltzmann constant, and $T$ is the absolute temperature. Eq. (6.3) is related to $T_{a}$ from Eq. (5.19), where $A(h k l ; T)=\frac{-T_{a}}{T_{c}}$. The difference between Eq. (6.3) and Eq. (5.19) is because Eq. (6.2) treats the harmonic thermal vibrations of the core and bonding electrons separately, whereas in Eq. (5.17) the amplitude of the harmonic vibrations are indistinguishable.

In a later study Hastings et al.[16] verified the anharmonic behavior of the Si core 
with temperature dependent measurements of the "thermally activated" $(442)_{C}$ and $(622)_{C}$ neutron diffraction forbidden reflections. The term "thermally activated" is used here because the intensity increases with temperature, rather than decreasing as seen with the $(222)_{C}$ reflection. The integrated intensity of the $(442)_{C}$ and $(622)_{C}$ reflections was found to increase as the anharmonic thermal vibrations grew with higher temperature, which is in agreement with the single-atom Einstein potential of Dawson and Willis[62]. Their results indicated that the temperature dependence can be described sufficiently by a potential that includes just the first anharmonic term, which is the third order displacement term in Eq. (5.13).

With the advent of high brilliance synchrotron X-ray radiation sources Mills and Batterman[18] were able to not only improve upon the results of previous studies but also accessed very weak higher index reflections. The results from X-ray diffraction measurements at a single temperature of the $(442)_{C}$ and $(622)_{C}$ forbidden reflections in both $\mathrm{Si}$ and Ge were in good agreement with the Dawson formalism[54, 61], but there was some discrepancy with Dawson's predicted values. Interestingly, the experimentally determined Si structure factors were consistently slightly larger than the Dawson predicted values[54]. Whereas the Ge structure factors were smaller than the expected values[61]. These results suggest that the bonding electron density is sharper in between the $\mathrm{Si}$ atoms and more distributed between the Ge atoms than what is predicted using Dawson's values. The departure from the predicted values can be explained when considering that $\mathrm{Si}$ and Ge have the same number of bonding electrons but there is a significant difference between their core charges.

Forbidden Bragg reflection measurements provide important information about the fine details of the charge density distribution of non-centrosymmetric atoms in crystals. Only the deviations in charge density distribution from spherical symmetry contribute to the forbidden Bragg reflection, like the charge density distribution from 
asymmetric bonding and anharmonic thermal effects, which accounts for a small portion of the total atomic charge. Whereas in allowed reflections the anisotropic part of the charge density distribution is obscured by the scattering contribution from the significantly greater isotropic component. Thus, forbidden Bragg reflections provide a unique opportunity to examine the subtle features of the charge density distribution. However, the difficulty of these experiments cannot be overstated. Therefore, it is worth highlighting some of the experimental challenges that are associated with diffraction measurements of forbidden reflections, including in the studies discussed above.

- The intensity of forbidden Bragg reflections are weak - several orders of magnitude weaker than allowed reflections.[57]

- Phase information is lost in diffraction measurements. Therefore, a combination of accurate temperature dependent X-ray and neutron diffraction measurements are required to extract the contributions of the anisotropic charge density distribution.[18]

- These studies depended on accurate temperature dependent integrated intensity measurements. For example, as was noted by Roberto et al.[66], faulty temperature readings in an earlier diffraction experiment[77] resulted in a misinterpretation of the data.

- Forbidden Bragg reflections, due to their inherent weak intensity, are vulnerable to contamination, such as multiple scattering events. Contamination of the measured reflection would result in misinterpretation of the charge density distribution, therefore it is important to avoid it.[13]

These are just a few examples and is not an exhaustive list of the experimental 
challenges that faced earlier studies.

\subsection{Renewed Interest and Recent Progress}

Recent technological advances have catalyzed a renewed interest in charge density. For example, the combination of, advancements in detectors including the availability of position-sensitive two-dimensional detectors have allowed for more complete experimental data sets; the brighter and more stable third-generation synchrotron Xray sources allow for more accurate measurements; and improved computing power; allows modern experiments to measure and process more information, and use models that have greater complexity.[50,74, 75] Consequently, modern experiments can challenge conventional theoretical models and improve on their shortcomings.

Recent studies are challenging conventional theoretical charge density models,[79] which uses a multi-polar treatment of the charge around a spherical core. The deficiencies of the conventional model only started to become apparent in the late 1990s when modern detector techniques were introduced, because up until then the accuracy of the data was insufficient to shed light on the shortcomings of the data. [50] For example, a recent synchrotron powder diffraction study of carbon diamond by Bindzus et al.[73] revealed that the conventional model that treated the core electron density distribution as an inert sphere was insufficient to explain their highly accurate data. Their experimental results revealed finer features about the electron density distribution including a deformation of the core electron density distribution, which is associated with the covalent bond configuration. Another recent charge density study that used X-ray powder diffraction measurements from a third-generation synchrotron source, Fisher et al.[76] examined the structure factors of carbon diamond and silicon. The experimental results revealed that the covalent bonding configu- 
ration in both elemental species perturb the overall charge density distribution and results in a deformed core shell.

It is worth noting that the deformations to the core electron density distribution mentioned above and the charge anisotropy from anharmonic thermal vibrations discussed in Section 6.3 are oriented in the same direction in the crystal. The thermal dependence of the core charge density distribution determined by Bindzus et al.[73] and Fisher et al.[76] is yet unknown. Therefore, the factors that dictate the deformation to the core electron density distribution are yet to be determined. In other words, at this time the following question remains unresolved: Is the core density deformation due to the influence from the covalent bonds, or is it due to anharmonic thermal vibrations of the core, or a combination of the two? With sufficient care and novel methods, subsequent X-ray scattering studies have the potential to not only resolve this outstanding question but also provide new insight on the electron density distribution in other crystals. 


\section{Chapter 7}

\section{Forbidden Bragg Reflections and Charge Density Distributions in X-ray CTRs}

\section{$7.1 \quad$ Introduction}

Forbidden Bragg reflections appear in our X-ray specular reflectivity, as seen in the reflectivity from $\mathrm{Si}(111)_{C} 7 \mathrm{x} 7$ and thin $\mathrm{Ag}$ film on $\mathrm{Si}(111)_{C} 7 \mathrm{x} 7$ in Fig. 7.1. The $\mathrm{Si}(222)_{C}$ forbidden reflection is seen at $L_{H}=6$ in both samples. The appearance of forbidden Bragg reflections complicates the interpretation and analysis of the scattering along crystal truncation rods (CTRs) because they appear in regions that contain important surface structure information. However, current CTR models, including specular reflectivity, do not include the forbidden Bragg reflection. For example, both best-fit curves to the conventional spherical charge distribution model in Fig. 7.1 are unable to explain the peak and the data surrounding it. In surface science the forbidden Bragg reflection is addressed by working around the sharp peak — often 
skipping over the region of reciprocal space that contains the forbidden reflection. Alternatively, in this work we have determined how to handle forbidden reflections in X-ray CTRs. In doing so, we have not only discovered a novel interference effect that allows - for the first time - one to determine both the amplitude and phase of the scattering from the forbidden reflection. But also, using the crystal surface, we have discovered a method for experimentally determining the bulk non-spherical electron density distribution, including the structure of the covalent bonds, from the continuous scattering between the Bragg reflections.

\subsection{Crystal Truncation Rods (CTRs)}

The scattering from a semi-infinite crystal will give rise to constructive interference in the form of Bragg peaks. Additional features are introduced to the scattering when the crystal is abruptly terminated with a flat surface, which is referred to as crystal truncation rods (CTRs). CTRs have been determined[80, 81] and discussed[42, 8284] elsewhere. Here we briefly review this type of scattering.

A cleaved crystal can be represented mathematically, in real-space, as the convolution of the bulk crystal density distribution, $\rho(z)$, and a step function, $h(z)$, where $z$ is perpendicular to the surface.[42] In reciprocal-space, the presence of a sharp terminated surface results in streaks of continuous scattering that span between the bulk Bragg peaks in the direction perpendicular to the surface. These surface morphology sensitive streaks of scattering are referred to as crystal truncation rods. The X-ray specular reflectivity rods in Fig. 7.1 are a particular example of CTRs, where due to the geometry the out-of-plane structure is probed. A ribbon of intensity can be

seen that extends perpendicular to the surface (along $L_{H}$ ) and drapes between the $\operatorname{Si}(000)_{C}, \operatorname{Si}(111)_{C}$, and $\mathrm{Si}(333)_{C}$ Bragg peaks. 


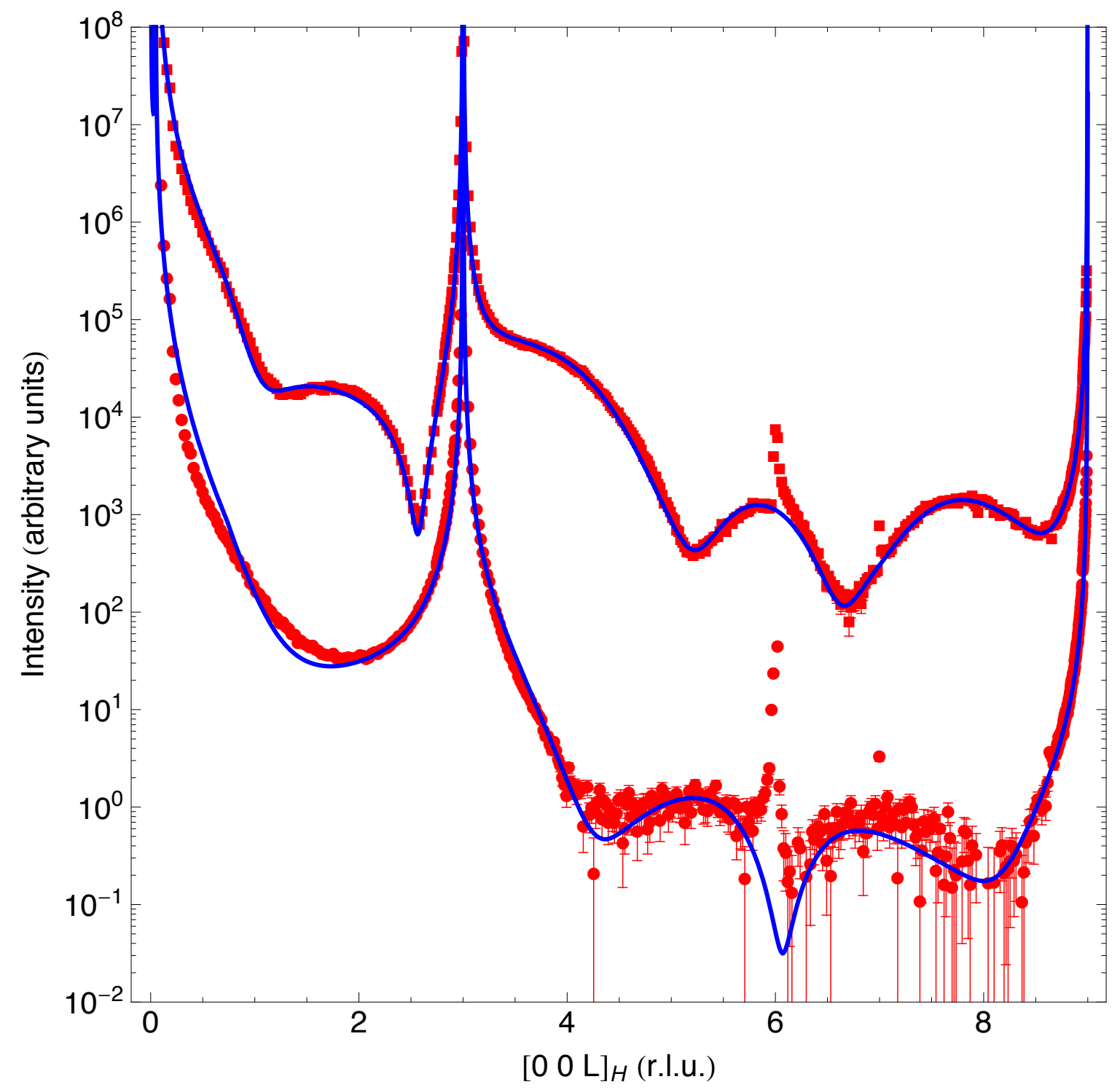

Figure 7.1: Best-fits of the spherical charge density distribution model to the specular X-ray reflectivity data of (bottom) bare $\mathrm{Si}(111)_{C} 7 \times 7$ and (top) thin Ag film on $\mathrm{Si}(111)_{C} 7 \times 7$. The $\mathrm{Si}(000)_{C}, \mathrm{Si}(111)_{C}$, and $\mathrm{Si}(333)_{C}$ Bragg peaks are located at $L_{H}=0,3$, and 9, respectively. All samples show the $\mathrm{Si}(222)_{C}$ forbidden Bragg reflection at $L_{H}=6$. Conventional spherical charge density distribution models are unable to explain the data around the forbidden reflection. 
The general form of the CTR scattering amplitude is[42]

$$
A_{C T R}=\frac{1}{1-e^{-i Q_{z} c}}
$$

where $c$ is the vertical lattice constant and $Q_{z}$ is the vertical component of the wavevector transfer. The scattering amplitude of a truncated crystal is determined by the product of the CTR and the bulk crystal scattering amplitudes, $A_{B u l k} A_{C T R}$.

\subsection{1 (111) $C$ Terminated Diamond Crystal Structure CTR}

The scattering amplitude of the $(111)_{C}$ terminated diamond crystal structure is the product of the amplitudes from the bulk, Eq. (5.41), and the CTR, Eq. (7.1), and expressed in hexagonal coordinates is

$$
\begin{aligned}
A_{D i a-C T R} & =F_{D i a} A_{C T R} \\
& =\left[f^{A}(\vec{Q})+f^{B}(\vec{Q}) e^{-i \vec{Q} \cdot \vec{r}_{A}}\right]\left[1+e^{-i \vec{Q} \cdot \vec{r}_{1}}+e^{-i \vec{Q} \cdot 2 \vec{r}_{1}}\right]\left[\frac{1}{1-e^{-i Q_{z} c_{H}}}\right],
\end{aligned}
$$

where $Q_{z} c_{H}=2 \pi L_{H}$.

Using the $\gamma$-formalism from Eq. (5.28), the temperature dependence is incorporated in the scattering amplitude by replacing the atomic form factors in Eq. (7.2) with

$$
\begin{aligned}
& f^{A}(\vec{Q})=\bar{f}_{c} T_{c} \gamma(\vec{Q}) \text { and } \\
& f^{B}(\vec{Q})=\bar{f}_{c} T_{c} \gamma(\vec{Q})^{*} .
\end{aligned}
$$

Therefore the temperature dependent scattering amplitude of the $(111)_{C}$ terminated 
diamond crystal structure is written as

$$
\begin{aligned}
A_{\text {Dia-CTR }}^{\prime}= & \bar{f}_{c} T_{c}\left[\gamma(\vec{Q})+\gamma(\vec{Q})^{*} e^{-i 2 \pi\left(\frac{2}{3} H_{H}+\frac{1}{3} K_{H}+\frac{1}{12} L_{H}\right)}\right] \times \\
& \frac{\left[1+e^{-i 2 \pi\left(\frac{2}{3} H_{H}+\frac{1}{3} K_{H}+\frac{1}{3} L_{H}\right)}+e^{-i 4 \pi\left(\frac{2}{3} H_{H}+\frac{1}{3} K_{H}+\frac{1}{3} L_{H}\right)}\right]}{1-e^{-i 2 \pi L_{H}}},
\end{aligned}
$$

It is convenient to use specular reflectivity to examine the interaction of the nonspherical charge density distribution with the CTR, because the specular geometry is less complicated than in-plane CTRs, (Specular reflectivity was also used to study the Si nanowire samples in Chapter 2. Though in that study the range of the specular reflectivity was significantly limited.) Specular reflectivity is a special case of CTRs where $H_{H}=K_{H}=0$. In this geometry the in-plane structure is integrated and the out-of-plane structure is probed. In the specular reflectivity geometry the scattering amplitude from Eq. (7.2) reduces to

$$
A_{\text {Dia-Spec }}=\left[f^{A}(\vec{Q})+f^{B}(\vec{Q}) e^{-i 2 \pi\left(\frac{1}{12} L_{H}\right)}\right] \frac{\left[1+e^{-i 2 \pi\left(\frac{1}{3} L_{H}\right)}+e^{-i 4 \pi\left(\frac{1}{3} L_{H}\right)}\right]}{1-e^{-i 2 \pi L_{H}}} .
$$

Similarly, the temperature dependent scattering amplitude from Eq. (7.4) reduces to

$$
A_{\text {Dia-Spec }}^{\prime}=\bar{f}_{c} T_{c}\left[\gamma(\vec{Q})+\gamma(\vec{Q})^{*} e^{-i 2 \pi\left(\frac{1}{12} L_{H}\right)}\right] \frac{\left[1+e^{-i 2 \pi\left(\frac{1}{3} L_{H}\right)}+e^{-i 4 \pi\left(\frac{1}{3} L_{H}\right)}\right]}{1-e^{-i 2 \pi L_{H}}} .
$$

In the following sections we examine the behavior of specular reflectivity and its components, as well as their interactions with spherical and non-spherical charge density distributions. 


\subsubsection{Specular Reflectivity with Spherical Charge Density Distribution}

Before we discuss the implications of non-spherical charge density distribution on the scattering it is helpful to examine the contributions from the spherical charge density distribution. Figure 7.2 shows the calculated specular reflectivity of an ideally terminated $(111)_{C}$ diamond crystal structure with spherically symmetric charge density distribution, as well as its reflectivity components from the diamond doublelayer, $\left|F_{D L}\right|^{2}$, and the ideally terminated FCC lattice, $\left|A_{C T R} F_{F C C}\right|^{2}$. When the charge density distribution is symmetric, the atomic form factors in Eq. (7.5) are indistinguishable and $f^{A}(\vec{Q})=f^{B}(\vec{Q})=f(Q)$, or in other words $\operatorname{Im}(\gamma(\vec{Q}))=0$. For these curves, the atomic form factor is taken as $f(Q)=1 .\left|F_{D L}\right|^{2}$ and $\left|A_{C T R} F_{F C C}\right|^{2}$ are calculated from the first and second terms in Eq. (7.5), respectively.

The calculated reflectivity curves are consistent with the conventional selection rules from Eq. (5.23) and (5.26). The FCC Bragg peaks appear at multiples of $L_{H}=3 n$, where $n$ is an integer. The $(000)_{C},(111)_{C},(222)_{C},(333)_{C}$, and $(444)_{C}$ Bragg peaks are located at $L_{H}=0,3,6,9$, and 12, respectively, while the $(222)_{C}$ peak is extinguished for the diamond crystal structure. The ideally terminated surface gives rise to a CTR, where a ribbon of non-zero intensity spans between the Bragg peaks along $L_{H}$ and perpendicular to the surface. The reflectivity component from the DL unit cell structure, $\left|F_{D L}\right|^{2}$, produces broad oscillations along $L_{H}$ and when the charge density distribution is spherically symmetric its intensity goes to zero when $L_{H}=6(2 n-1)$, where $n$ is an integer. As for the diamond crystal structure reflectivity curve, $\left|A_{\text {Dia-Spec }}\right|^{2}$, the Bragg peaks from the FCC lattice remain present except for when $L_{H}=6(2 n-1)$. These Bragg peaks are extinguished by the diamond double-layer structure component. This conventional treatment of the bulk $\operatorname{Si}(111)_{C}$ structure does not explain the peak at $L_{H}=6$ in Fig. 7.1. 


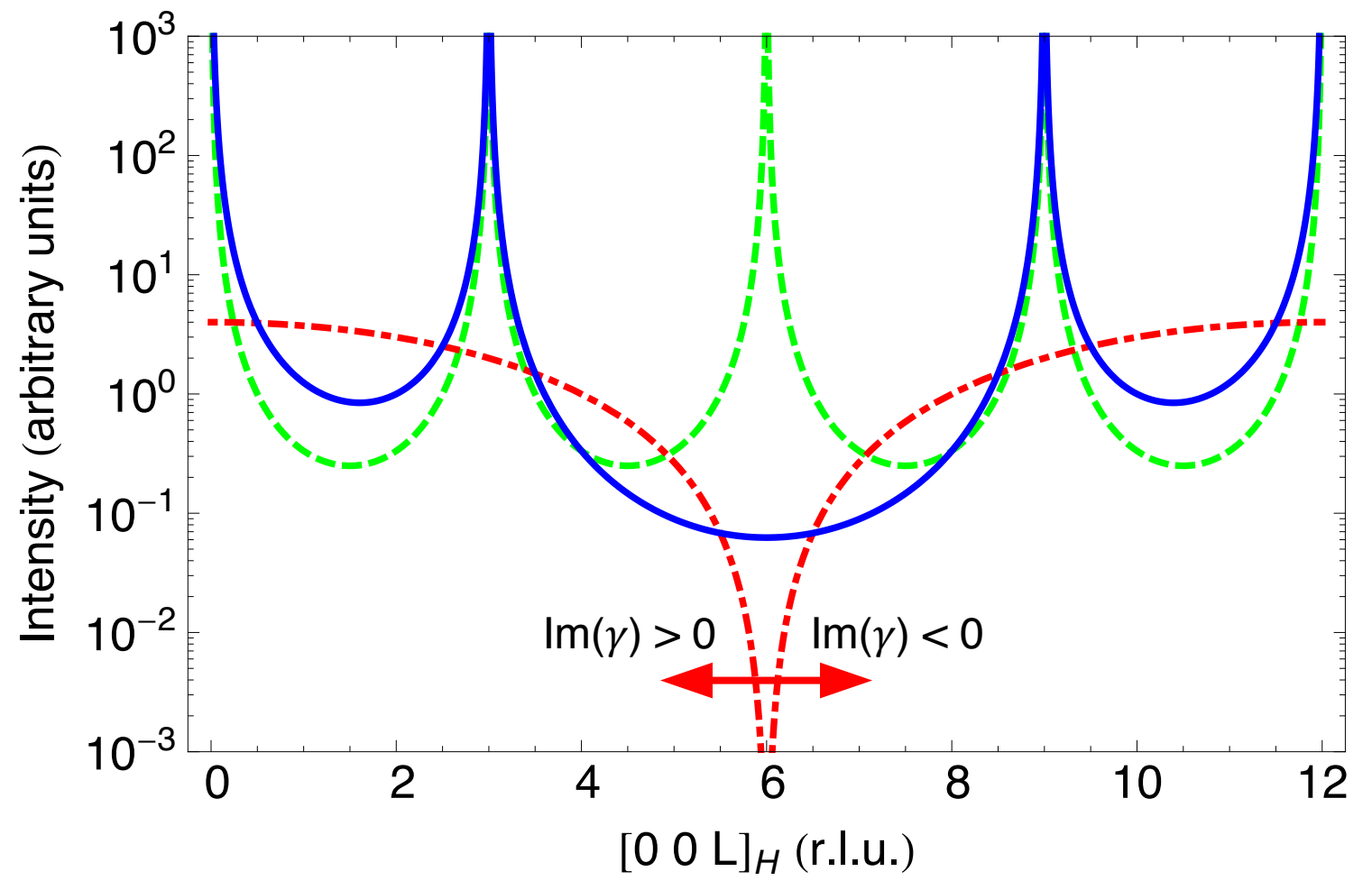

Figure 7.2: Calculated specular X-ray reflectivity of an ideally terminated $(111)_{C}$ diamond crystal structure (solid blue curve) from Eq. (7.5) with spherical charge density distribution (i.e. $f^{A}(\vec{Q})=f^{B}(\vec{Q})=f(Q)$ or $\operatorname{Im}(\gamma(\vec{Q}))=0$ ) along with its components. The dashed green curve is the specular reflectivity for an ideally terminated $(111)_{C}$ FCC crystal, $\left|A_{C T R} F_{F C C}\right|^{2}$, which is determined from the second term in Eq. (7.5). The dot-dashed red curve is the intensity from the diamondstructure double-layer component, $\left|F_{D L}\right|^{2}$, which is determined from the first term in Eq. (7.5). For these curves, the atomic form factor is taken as $f(Q)=1 .\left|F_{D L}\right|^{2}$ experiences a phase shift along $L_{H}$ when the charge density distribution is anisotropic $\operatorname{Im}(\gamma) \neq 0$. 


\subsubsection{Specular Reflectivity with Non-Spherical Charge Den- sity Distribution}

When the charge density distribution is non-spherical the A- and B-sites are distinguishable (i.e. $f^{A}(\vec{Q}) \neq f^{B}(\vec{Q})$ and $\operatorname{Im}(\gamma) \neq 0$ ) the form factors in Eq. (7.5) are

complex, which introduces a phase on $\left|F_{D L}\right|^{2}$ that results in a shift along $L_{H}$. In other words, this phase shift effectively translates the $\left|F_{D L}\right|^{2}$ curve in Fig. 7.2 along the direction perpendicular to the surface, i.e. along $L_{H}$. This phase shift impacts the reflectivity in two important ways:

1. $\left|F_{D L}\right|^{2}$ no longer extinguishes the FCC Bragg peaks at $L_{H}=6(2 n-1)$, thus allowing the forbidden reflection to be observed, and

2. the minima of $\left|F_{D L}\right|^{2}$ extinguishes the intensity of the truncation rod, which produces a cusp adjacent to the $L_{H}=6(2 n-1)$ forbidden reflection.

The former can be observed with or without the presence of a CTR. Though, the later requires the a truncation rod from an atomically smooth crystal surface.

Either a positive or negative phase shift will result in an observable forbidden reflection peak. Diffraction techniques have been previously used to experimentally measure these peaks (see Chapter 6). Though, this technique measures the integrated intensity and because either a positive or negative phase shift results in the forbidden Bragg reflection, diffraction is insensitive to the sign of the phase shift, regardless of the surface morphology.

However, when the crystal surface is smooth on the atomic scale, the influence from the phase shift is broader than just the peak and extends along the CTR. Interference between the waves from the surface and the bulk forbidden Bragg reflection produce an adjacent cusp, which can be measured using CTR techniques, and is seen in the reflectivity data in Fig. 7.1. 
To help us understand the impact of the phase shift in $\left|F_{D L}\right|^{2}$ we turn to the calculated reflectivity curves in Fig. 7.3. When $\operatorname{Im}(\gamma)>0,\left|F_{D L}\right|^{2}$ experiences a phase shift towards the origin and results in the reflectivity curve shown in Fig. 7.3(a). Similarly, $\left|F_{D L}\right|^{2}$ is shifted away from the origin when $\operatorname{Im}(\gamma)<0$ and results in the calculated curve shown in Fig. 7.3(b).

The phase shift in the diamond double-layer structure, including its sign, can be explained by the anisotropic charge density distribution. More specifically, when the anharmonic thermal vibrations dominate the anisotropic charge density, as in $\operatorname{Im}(\gamma)>0$, the charge deformation is oriented toward the void opposite the bonds, as illustrated in Fig. 6.2(b). In this case the cusp will be adjacent to the forbidden reflection on the side closer to the origin, as seen in Fig. 7.3(a). Similarly, when the asymmetric bonding distribution dominates $(\operatorname{Im}(\gamma)<0)$ the net anisotropic charge density is directed toward the bonds, as illustrated in Fig. 6.2(a), the cusp will be on the side away from the origin, as seen in Fig. 7.3(b). Furthermore, considering that the proximity of the cusp to the forbidden reflection is related to the magnitude of the phase shift, the deflection of the cusp from the peak can be used to determine the magnitude of the anisotropic charge density.

For the first time we have shown, with a single X-ray measurement, that phase information is extracted from the cusp adjacent to the diamond crystal structure forbidden Bragg reflection. The forbidden reflection from the bulk interferes with the scattering from the atomically smooth crystal surface and, as a result, influences the scattering along the CTR beyond the forbidden reflection peak. In other words, when the anisotropic charge density is non-zero (i.e. $\operatorname{Im}(\gamma) \neq 0$ ) the first term in brackets of the total scattering amplitude in Eq. (7.6) experiences a phase shift and interferes with the last term, which produces a cusp adjacent to the peak along the crystal truncation rod. The interference between the scattered amplitudes of the bulk and 

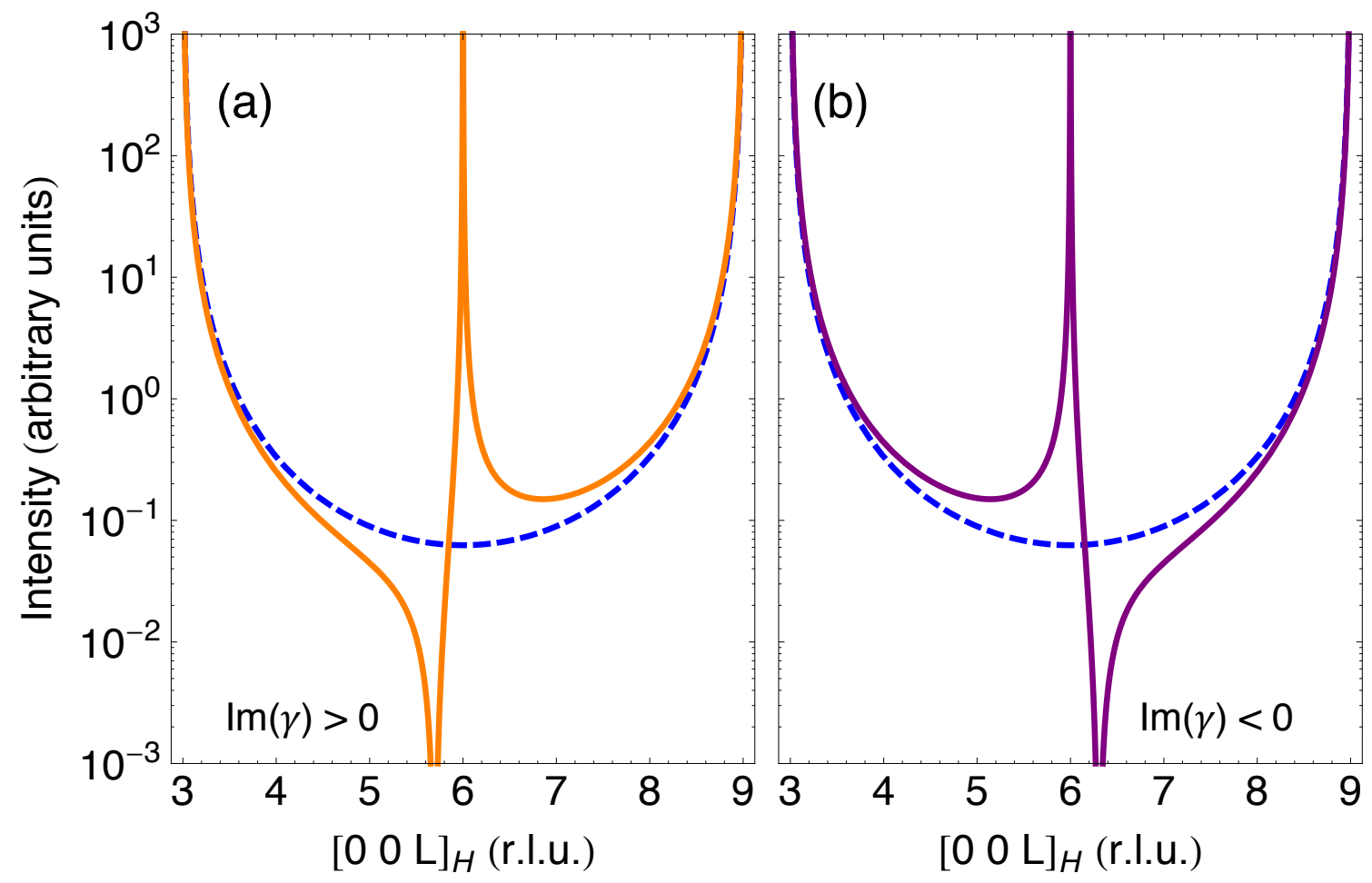

Figure 7.3: Calculated specular X-ray reflectivity, from Eq. (7.6), near the diamond crystal structure $(222)_{C}$ forbidden reflection (appearing here at $L_{H}=6$ ). For simplicity the atomic form factor and temperature factor are set to one, $\bar{f}_{c} T_{c}=1$. For comparison the dashed blue curve is the calculated intensity for the spherical charge density distribution model, where $\operatorname{Im}(\gamma)=0$. The two solid curves are the calculated intensities using the non-spherical charge density distribution model (i.e. $\operatorname{Im}(\gamma) \neq 0$ ). The solid curves in (a) and (b) illustrate the behavior of the forbidden reflection cusp when the anisotropic charge density is dominated by the anharmonic thermal vibrations (i.e. $\operatorname{Im}(\gamma)>0$ ) and the asymmetric bonding charge density distribution (i.e. $\operatorname{Im}(\gamma)<0)$, respectively. For simplicity $\gamma=$ constant was used to generate the solid curves in (a) and (b), where $\gamma=1+i 0.08$ and $\gamma=1-i 0.08$, respectively. 
the surface permit one to determine both amplitude and phase information of the bulk crystal using CTR measurement techniques.

\subsubsection{In-Plane CTRs}

Forbidden Bragg reflections also occur in non-specular geometry CTRs. Calculated examples of in-plane CTRs are shown in Fig. 7.4. Intensities for the $\left[\begin{array}{lll}1 & 0\end{array}\right]_{H}$, $\left[\begin{array}{lll}2 & 0 & \mathrm{~L}\end{array}\right]_{H}$, and $\left[\begin{array}{lll}3 & 0 & \mathrm{~L}\end{array}\right]_{H}$ rods are shown at $100 \mathrm{~K}$ and $900 \mathrm{~K}$. Here we use $\operatorname{Si}(111)_{C}$ as

an example. The temperature dependent intensities, $\left|A_{S i(11)_{C}-C T R}^{\prime}\right|^{2}$, are calculated from Eq. (7.4), using bulk Si structure and charge density distribution parameter values. Tabulated[64] Si values were used for $\bar{f}_{c}^{S i}(Q)$. Previously determined charge density distribution and temperature parameters, from Tables 4.1 and 5.2, respectively, were used to calculate $\gamma^{S i}(\vec{Q})$ and $T_{c}^{S i}$. By comparison, in Section 7.2.3 we set $\gamma=$ constant for simplicity and to demonstrate the impact on the scattering when $\operatorname{Im}(\gamma) \neq 0$. In this section we will examine the $\vec{Q}$ and temperature dependence of $\gamma^{S i}(\vec{Q})$ at Si forbidden Bragg reflections in several in-plane CTRs.

Forbidden Bragg reflections appear at one of three locations along a CTR for a $(111)_{C}$ terminated diamond crystal structure, within the range of $L_{H}=0$ and 13: $(\mathrm{H} \mathrm{K} \mathrm{2})_{H},(\mathrm{H} \mathrm{K} 6)_{H}$, and $(\mathrm{H} \mathrm{K} \mathrm{10})_{H}$. An example of each is shown in Fig. 7.4. The $(244)_{C},(\overline{2} 22)_{C}$, and $(\overline{2} 44)_{C}$ Si forbidden Bragg reflections are located at (a and b) $\left(\begin{array}{lll}1 & 0 & 10\end{array}\right)_{H}$, (c and d) $\left(\begin{array}{lll}2 & 0 & 2\end{array}\right)_{H}$, and (e and f) $\left(\begin{array}{llll}3 & 0 & 6\end{array}\right)_{H}$, respectively. The plots on the right show magnified views of the region near the forbidden reflections.

These calculated curves are helpful for inspecting the behavior of the interference between the forbidden Bragg reflection and the in-plane CTRs. A cross-over between $-\operatorname{Im}(\gamma)$ and $+\operatorname{Im}(\gamma)$ can be seen in the interference between the $\left(\begin{array}{lll}2 & 4\end{array}\right)_{C}$ forbidden reflection and the CTR in Fig. 7.4(a and b). At low temperature the asymmetric 

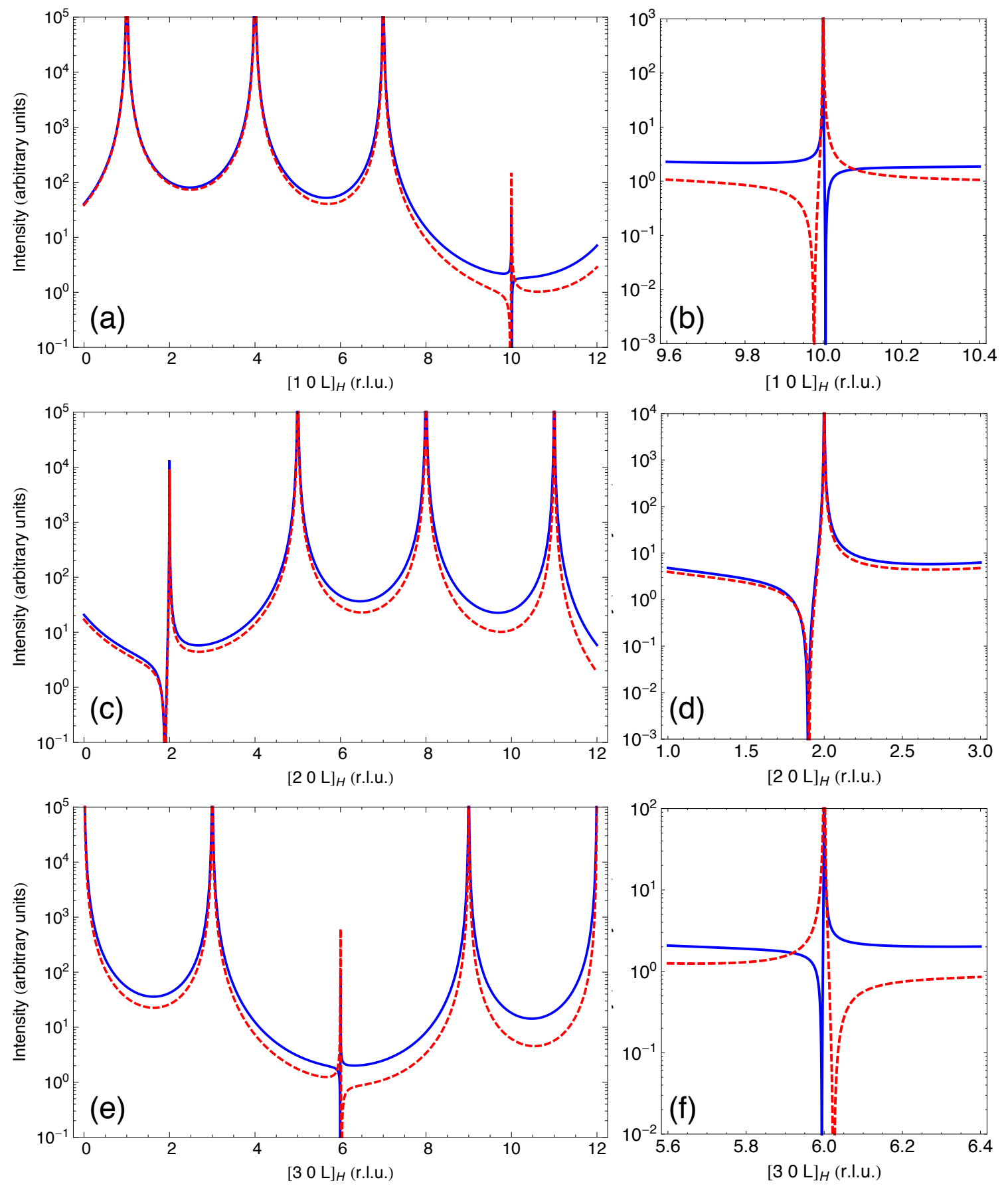

Figure 7.4: Calculated intensities of the ((a) and (b)) $[10 \mathrm{~L}]_{H}$, ((c) and (d)) $[20 \mathrm{~L}]_{H}$, and $((\mathrm{e})$ and $(\mathrm{f}))\left[\begin{array}{ll}3 & 0 \mathrm{~L}\end{array}\right]_{H}$ CTRs from the $\mathrm{Si}(111)_{C}$ crystal structure at (solid blue curves) $100 \mathrm{~K}$ and (dashed red curves) $900 \mathrm{~K}$. The $\operatorname{Si}(244)_{C}, \operatorname{Si}(\overline{2} 22)_{C}$, and $\operatorname{Si}(\overline{2} 44)_{C}$ forbidden reflections are located at ((a) and (b)) $\left(\begin{array}{lll}1 & 0 & 10\end{array}\right)_{H},((\mathrm{c})$ and (d) $)\left(\begin{array}{lll}2 & 0 & 2\end{array}\right)_{H}$, and $((\mathrm{e})$ and $(\mathrm{f}))\left(\begin{array}{lll}3 & 0 & 6\end{array}\right)_{H}$, respectively. (The three-dimensional dependence of the change in $\gamma^{S i}(\vec{Q})$ between $100 \mathrm{~K}$ and $900 \mathrm{~K}$ was also seen in Fig. 5.7.) 
bonding distribution dominates the anisotropic charge density and results in a cusp located away from the origin, while at high temperature the anharmonic vibrations dominate and cause the cusp to switch sides. On the other hand, the cusp associated with the $(\overline{2} 22)_{C}$ forbidden reflection, seen in Fig. $7.4(\mathrm{c}$ and $\mathrm{d})$ is located on the side closer to the origin. The orientation of the cusp is opposite to that of the (2 22 2 $)_{C}$ reflection because, as we discussed in Ch. 6, an odd number of negative cubic Miller indices causes the phase to switch sign due to reflection symmetry, i.e. $\operatorname{Im}(\gamma(-\vec{Q}))=-\operatorname{Im}(\gamma(\vec{Q}))$. Even at the elevated temperature of 900K the cusp remains on the same side of the peak because the asymmetric bonding significantly dominates the $\operatorname{Si}\left(\begin{array}{lll}2 & 2\end{array}\right)_{C}$ reflections at all temperatures. The strong contribution from the bonding distribution and negligible influence from the anharmonic vibrations were also exhibited in the temperature dependent curves of $\operatorname{Im}\left(\gamma^{S i}(222)_{C}\right)$ in Fig. 5.8. The cusp associated with $(\overline{2} 44)_{C}$, shown in Fig. 7.4(e) and (f), has the opposite orientation from the $\left(\begin{array}{lll}2 & 4 & 4\end{array}\right)_{C}$ forbidden reflection because one of the cubic Miller indices is negative.

The CTRs shown in Fig. 7.4 probe the crystal structure along different orientations therefore the scattering contributions from the non-spherical charge density distributions will also be different. For example, the $\left(\begin{array}{lll}2 & 2 & 2\end{array}\right)_{C}$ reflections is oriented along the $\langle 111\rangle_{C}$ direction of the crystal. In this geometry the covalent bond between the $\mathrm{A}$ and $\mathrm{B}$ sites are probed along the bonding axes, where the radial density of the asymmetric charge density distribution is expected to be the greatest. Therefore as predicted the $\left(\begin{array}{lll}\overline{2} & 2 & 2\end{array}\right)_{C}$ cusp has the greatest deflection away from the peak at low temperature. Whereas the deflection of the cusps shown in Fig. 7.4(b) and (f), at low temperature, are small by comparison because the orientation of the $\left(\begin{array}{lll}4 & 4\end{array}\right)_{C}$ scattering vector relative to the bonding distribution.

It is convenient to use maps of reciprocal space to help navigate the locations of the 
forbidden reflections. Figure 7.5(a) and (b) shows two- and three-dimensional maps, respectively, of the first sextant for the $(111)_{C}$ diamond crystal structure forbidden reflections in hexagonal coordinates. The colors and shapes indicate the location of the forbidden reflection along the CTRs. For example, the specular reflectivity rod, which is located at $\left[\begin{array}{lll}0 & 0 & \mathrm{~L}\end{array}\right]_{H}$, is labeled with a green square and therefore has a forbidden reflection at $\left(\begin{array}{lll}0 & 0 & 6\end{array}\right)_{H}$. The in-plane CTRs from Fig. 7.4 are located near the specular reflectivity rod along the $H_{H}$ axis. The $\left[\begin{array}{lll}1 & 0 & \mathrm{~L}\end{array}\right]_{H},\left[\begin{array}{lll}2 & 0 & \mathrm{~L}\end{array}\right]_{H}$, and $\left[\begin{array}{lll}3 & 0 & \mathrm{~L}\end{array}\right]_{H}$ rods are coded with a yellow diamond, blue circle, and green square, which correspond to forbidden Bragg reflections that occur at $\left(\begin{array}{lll}1 & 0 & 10\end{array}\right)_{H},\left(\begin{array}{lll}2 & 0 & 2\end{array}\right)_{H}$, and $\left(\begin{array}{lll}3 & 0 & 6\end{array}\right)_{H}$, respectively.

There are several unmarked rods because their forbidden reflections are truly absent in the range between $L_{H}=0$ and 13 . These absent forbidden reflections correspond to scattering geometries where at least one of the cubic Miller indices are zero - also discussed in Chapter 5.2.1. It is helpful to use three-dimensional maps like Fig. 7.5(b) to visualize the locations of the truly extinct forbidden reflections. Three trisecting planes are formed when at least one of the cubic Miller indices are zero. One of these extinction planes is seen in the three-dimensional map and is oriented into the page on an angle and tilted from vertical and nearly bisects the sextant.

\subsection{Experimental}

In-Situ X-ray reflectivity experiments were performed in an ultra-high vacuum growth and analysis chamber (base pressure of $1 \times 10^{-10}$ Torr) that is integrated with a $\psi$-diffractometer at the 6IDC beam line at the Advance Photon Source, Argonne National Laboratory. The photon energy was $16.2 \mathrm{keV}$ for this study. A Bicron de- 

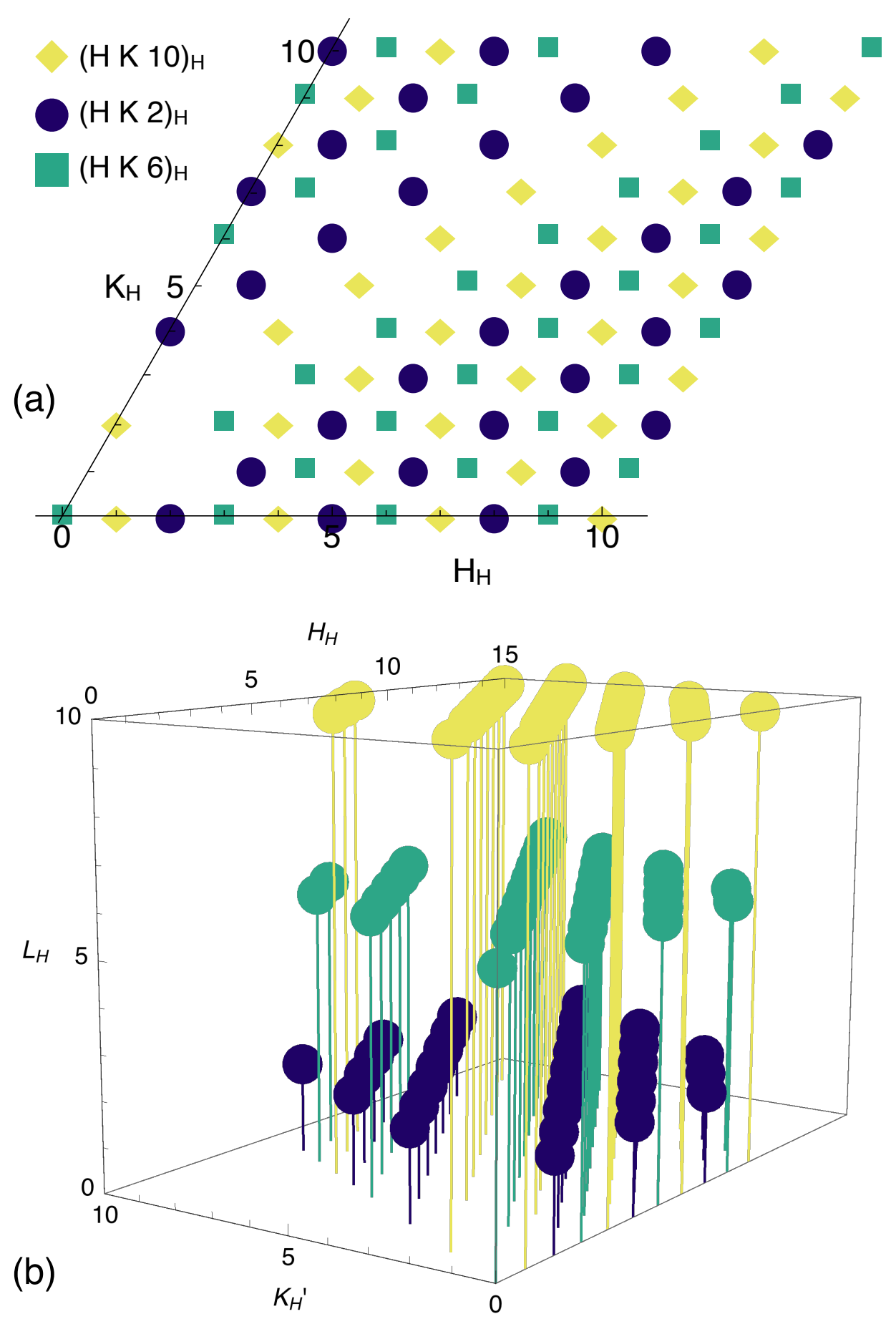

Figure 7.5: (a) Two- and (b) three-dimensional maps of the $(111)_{C}$ diamond crystal structure forbidden reflections in hexagonal coordinates. The colors (and shapes in (a)) indicate the location of the forbidden Bragg reflection along the CTR. Example calculated CTR curves are shown in Fig. 7.4. $K_{H}$ is not perpendicular to $H_{H}$, therefore in (b) the projection of $K_{H}$ onto the Cartesian axis is labeled " $K_{H^{\prime}}$ ". 
tector was used to collect the data. A dedicated $\frac{\lambda}{3}$ scaler channel was used and $\frac{\lambda}{3}$ contamination was not observed during the measurement of the forbidden reflection. Abnormalities in the background intensity data around the $\mathrm{Si}(222)_{C}$ forbidden reflection, $L_{H}=6 \pm 0.5$, such as contributions from the forbidden Bragg reflection, were corrected with a linear interpolation of the background intensity. We are confident that the background subtraction did not introduce artifacts into the final reflectivity data.

Commercially available $1 \mathrm{~mm}$-thick n-type $\mathrm{Si}(111)_{C}$ substrates were used in this study. The $\operatorname{Si}(111)_{C} 7 \times 7$ surfaces were prepared by flash annealing using established methods[85]. Ag was thermally deposited on the $\mathrm{Si}(111)_{C} 7 \times 7$ surface at $300 \mathrm{~K}$. The samples were at 300K during the specular X-ray reflectivity measurements. The temperature was monitored by a type-K thermocouple located on the sample holder. The Ag deposition rate was $1.1 \pm 0.1 \mathrm{ML} / \mathrm{min}$ and was determined by a commercial quartz crystal oscillator monitor that was calibrated by measuring layer-by-layer homoepitaxial growth of $\mathrm{Ag}(001)_{C}$. 1ML is one monolayer of $\mathrm{Ag}(111)_{C}$ and is equivalent to $1.38 \times 10^{15}$ atoms $/ \mathrm{cm}^{2}$. Three samples are presented in this study, a bare $\mathrm{Si}(111)_{C} 7 \times 7$ substrate, thin $\mathrm{Ag}$ film deposited on $\mathrm{Si}(111)_{C} 7 \times 7$, and thick $\mathrm{Ag}$ film deposited on $\mathrm{Si}(111)_{C} 7 \times 7$. The Miceli group[86] performed the X-ray measurements.

\subsection{Results}

Figure 7.6 shows best-fits to the X-ray specular reflectivity of three samples, which have distinctive surface morphologies: clean $\mathrm{Si}(111)_{C} 7 \mathrm{x} 7$, thin $\mathrm{Ag}$ film on $\mathrm{Si}(111)_{C} 7 \mathrm{x} 7$, and thick $\mathrm{Ag}$ film on $\mathrm{Si}(111)_{C} 7 \times 7$. The prominent peaks at $L_{H}=0,3$, and 9 correspond to the $\mathrm{Si}(000)_{C}, \mathrm{Si}(111)_{C}$, and $\mathrm{Si}(333)_{C}$ Bragg reflections, respectively, and the sharp peak at $L_{H}=6$ corresponds to the $\operatorname{Si}(222)_{C}$ forbidden reflection. 
The scattering between the Si Bragg peaks is noticeably evolving as Ag is added to the $\operatorname{Si}(111)_{C} 7 x 7$ surface. With sufficiently thick films the $\operatorname{Ag}(111)_{C}$ and $\operatorname{Ag}(222)_{C}$ Bragg peaks near $L_{H}=4$ and 8 become pronounced along the truncation rod and drown out the scattering from the Si7x7 surface structure. However, in the midst of the interference from the $\mathrm{Ag}$ film the $\mathrm{Si}(222)_{C}$ forbidden reflection is not extinguished, but rather the peak undergoes a symmetry flip due to the additional phase that is introduced by the Ag film. It is demonstrated by the quality of fits to the reflectivity data that our models are able to explain the flip in peak symmetry. The quality of fit across all the samples is impressive considering that the three model curves use the same non-spherical charge density distribution correction, $\gamma^{S i}(\vec{Q})$, keeping in mind their vastly differing surface morphologies. The analysis of each sample including the method for determining $\gamma^{S i}(\vec{Q})$ is discussed in greater detail below.

\subsubsection{Structure Models}

The samples used in this study are $\mathrm{Si}(111)_{C}$ with a $\mathrm{Si} 7 \mathrm{x} 7$ reconstructed surface and $\operatorname{Si}(111)_{C} 7 \times 7$ with thin and thick Ag films. The temperature dependent specular reflectivity scattering amplitude of bulk $\mathrm{Si}(111)_{C}$ is calculated from Eq. (7.6) and is expressed as

$$
\begin{array}{r}
A_{S i(111)_{C}-\text { Spec }}^{\prime}=\bar{f}_{c}^{S i}(Q) T_{c}^{S i}(Q)\left[\gamma^{S i}(\vec{Q})+\gamma^{S i}(\vec{Q})^{*} e^{-i 2 \pi \frac{1}{12} L_{H}}\right] \\
\times \frac{\left[1+e^{-i 2 \pi \frac{1}{3} L_{H}}+e^{-i 4 \pi \frac{1}{3} L_{H}}\right]}{1-e^{-i 2 \pi L_{H}}} .
\end{array}
$$

Tabulated[64] Si values were used for $\bar{f}_{c}^{S i}(Q) . \quad T_{c}^{S i}$ was calculated from Eq. (5.10) using previously determined temperature parameters from Table 5.2. Previously determined charge density distribution and temperature parameters, from Tables 4.1 

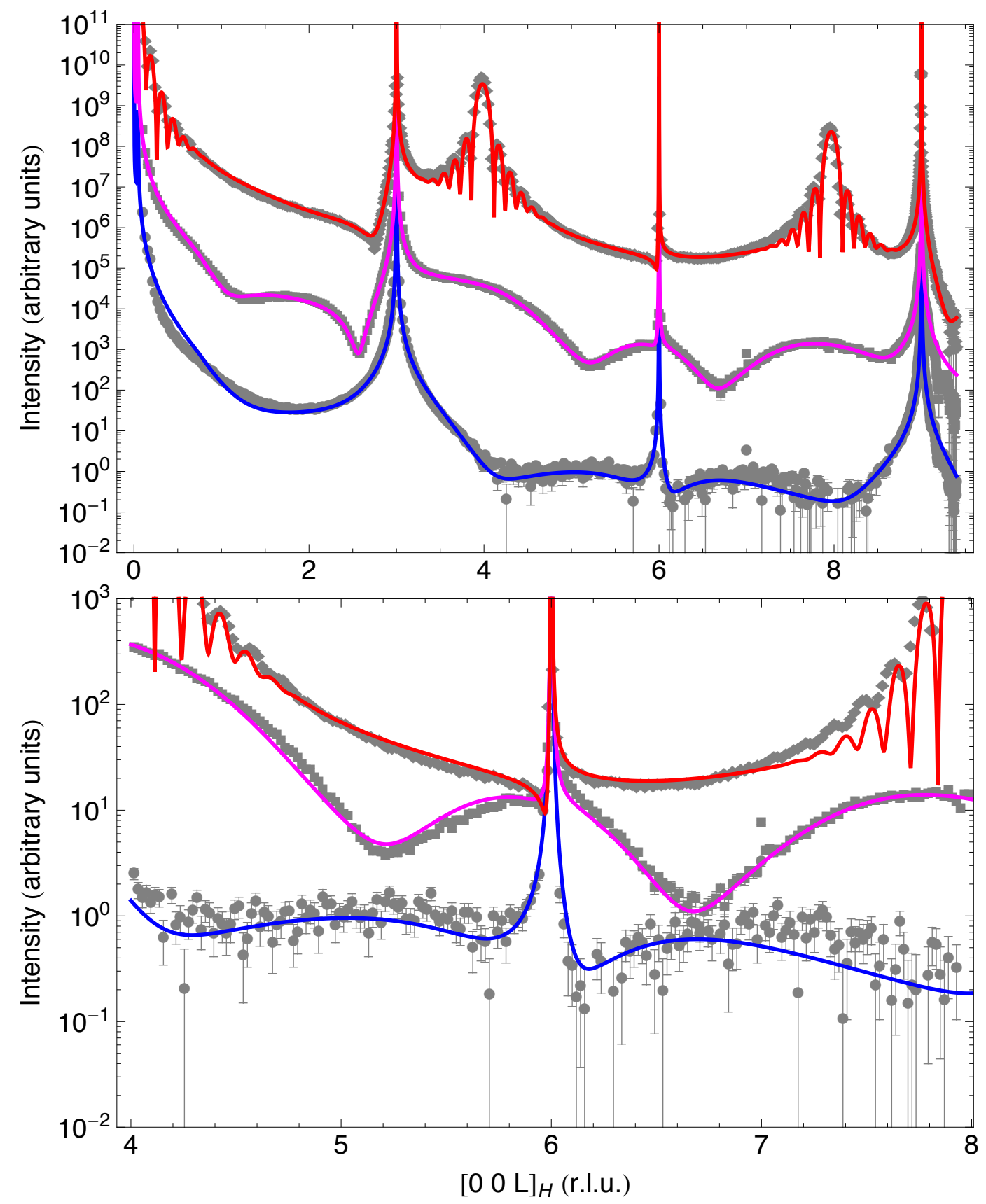

Figure 7.6: Best-fits to X-ray specular reflectivity data of three samples, from bottom to top: bare $\mathrm{Si}(111)_{C} 7 \times 7$, thin $\mathrm{Ag}$ film on $\mathrm{Si}(111)_{C} 7 \times 7$, and thick $\mathrm{Ag}$ film on $\mathrm{Si}(111)_{C} 7 \times 7$. (a) The intensities of each sample are offset for visual clarity. (b) Magnified view near the $\mathrm{Si}(222)_{C}$ forbidden reflection, located at $L_{H}=6$, with the intensities shown on an absolute scale. All samples show the $\mathrm{Si}(222)_{C}$ forbidden reflection at $L_{H}=6$. All of the curves shown use the same $\gamma^{S i}(\vec{Q})$, where $\operatorname{Im}\left(\gamma^{S i}(222)_{C}\right)=-0.034$. 
and 5.2, respectively, were used to initially calculate $\gamma^{S i}(\vec{Q}) \cdot \gamma^{S i}(\vec{Q})$ was then refined using experimental specular X-ray reflectivity data. More specifically, the values of $\alpha$ and $K_{3,22}$ were refined in this study. Whereas $L_{4,22}, \alpha_{t}$, and $\beta_{t}$ were fixed at the previously determined values because this study was not sensitive to these parameters. The temperature was constant during this study and the specular reflectivity from $\mathrm{Si}(111)_{C}$ is not sensitive to the centrosymmetric charge density distribution nor to the anharmonic thermal vibrations due to geometry.

Two versions of the X-ray specular reflectivity model for bulk $\operatorname{Si}(111)_{C}$ from Eq. (7.7) are used in this study:

1. Conventional spherical charge density distribution model, where $\gamma^{S i}(\vec{Q})=1+i 0$.

2. Non-spherical charge density distribution model, where $\operatorname{Im}\left(\gamma^{S i}(\vec{Q})\right) \neq 0$.

All other aspects of these two models are identical.

The general scattering amplitude from a diamond crystal structure with a surface reconstruction and a deposited film is

$$
A_{\text {total }}=\left(\rho_{\text {bulk }}\left(A_{\text {Dia-CTR }}+A_{\text {surface }}\right)+\rho_{\text {film }} A_{\text {film }}\right) V_{\text {sub }},
$$

where $A_{D i a-C T R}$ is the CTR amplitude from Eq. (7.2), $A_{\text {surface }}$ is the amplitude from the reconstructed surface structure, $A_{\text {film }}$ is the amplitude of the film, $\rho_{b u l k}$ and $\rho_{\text {film }}$ are areal densities, and $V_{s u b}$ is the substrate roughness.

$\operatorname{Si}(111)_{C}$ substrates with $7 \times 7$ reconstructed surfaces were used in this study. The temperature dependent specular scattering amplitude from the $\mathrm{Si}(111)_{C}$ is determined by Eq. (7.7). The calculation of $V_{\text {sub }}$, the specular scattering amplitude of the Si7x7 surface reconstruction and the Ag films are proved below.

The error bars for structural and charge density distribution parameters reported in this text were established by determining the range that the parameter could be 
varied while maintaining an acceptable fit to the data. The error bars for the X-

ray data are statistical in nature, where $\delta$ counts $=\frac{\sqrt{\text { counts }}}{\text { time }}$. Several experimental corrections were incorporated in our model, which include: resolution correction for the detector slit size, $\frac{1}{Q^{2}}$ for the Lorentz factor, linear polarization correction, and footprint correction for the illuminated area of the sample.

Yiyao Chen is acknowledged for helping with implementing the crystal structure models used in this work.

\section{Substrate Roughness}

The substrate roughness $[41,87]$ is expressed as

$$
\begin{aligned}
\left|V_{\text {sub }}\right|^{2} & =e^{-\left(2 \frac{\sigma}{d} \sin \frac{\vec{Q} \cdot \vec{R}}{2}\right)^{2}} \\
& =e^{-\left(2 \frac{\sigma}{d} \sin \frac{2 \pi\left(2 H_{H}+K_{H}+L_{H}\right)}{6}\right)^{2}}
\end{aligned}
$$

where $\sigma$ is the RMS of the substrate and $d=\frac{c_{H}}{3}$ is the $\operatorname{Si}(111)_{C}$ layer spacing.

\section{$\operatorname{Si}(111)_{C} 7 \times 7$ Reconstructed Surface}

The $\operatorname{Si}(111)_{C} 7 \mathrm{x} 7$ reconstructed surface consists of five Si layers: $2 \mathrm{~B}, 2 \mathrm{~A}, 1 \mathrm{~B}, 1 \mathrm{~A}$, and an Ad-atom layer. We will use the vertical structure layer occupancies that were determined by Robinson and Vlieg [88]. Layers 2B and 2A, which have 49 atoms in the unit cells, are not reconstructed and have the same structure as the bulk Si below. Layer $1 \mathrm{~B}$ has a dimer structure and is missing an atom at the origin; therefore 48 atoms are this unit cell. Layer 1A exhibits staking fault islands with only 42 atoms in the unit cell. Lastly, at the top of the reconstructed surface, there are only 12 atoms in the Ad-atom layer. The temperature dependent specular scattering amplitude for the reconstructed Si7x7 surface is determined by adding up the amplitudes of the five 
layers, [88]

$$
A_{7 x 7}^{\prime}=\bar{f}_{c}^{S i}(Q) T_{c}^{S i}(Q)\left(A_{2 B}+A_{2 A}+A_{1 B}+A_{1 A}+A_{\text {Ad-atom }}\right),
$$

where $\bar{f}_{c}^{S i}(Q)$ is the spherical atomic form factor for $\mathrm{Si}$, from Eq. (5.6), $T_{c}^{S i}(Q)$ is the thermal Debye Waller factor from Eq. (5.10), and $A_{n}$ are the amplitudes of the $n^{\text {th }}$ layer of the Si7x7 reconstructed surface.

$\bar{f}_{c}^{S i}(Q)$ is used for the surface structure to simplify the analysis, rather than a complex atomic form factor. The bond lengths of the Si7x7 atoms differ from that of the bulk Si atoms, which complicates the calculation of the non-spherical charge density distribution for these atoms. Considering that the scattering from the five Si7x7 layers is relatively weak, the contribution to the CTR from the non-spherical charge density distribution of the Si7x7 atoms will be limited, and therefore can be left out at this time.

In this study, specular reflectivity geometry $\left(H_{H}=K_{H}=0\right)$ was used to measure the $\operatorname{Si}(111)_{C} 7 \times 7$ structure. Therefore for simplicity, we can ignore the in-plane positions of the atoms and construct the Si7x7 structure model for the out-of-plane positions.

The specular scattering amplitude for each layer of the $\mathrm{Si}(111)_{C} 7 \times 7$ reconstructed surface in Eq. (7.10), including root-mean-square (RMS) roughness, was previously 
determined by the Miceli group[72, 89] and is expressed as

$$
\begin{aligned}
A_{2 B} & =e^{i \vec{Q} \cdot\left(\frac{c_{H}}{4}+z_{2 B}\right) \hat{z}} e^{-\frac{1}{2} Q^{2} \sigma_{2 B}^{2}} \\
A_{2 A} & =e^{i \vec{Q} \cdot\left(\frac{c_{H}}{3}+z_{2 A}\right) \hat{z}} e^{-\frac{1}{2} Q^{2} \sigma_{2 A}^{2}} \\
A_{1 B} & =\frac{48}{49} e^{i \vec{Q} \cdot\left(\frac{7 c_{H}}{12}+z_{1 B}\right) \hat{z}} e^{-\frac{1}{2} Q^{2} \sigma_{1 B}^{2}} \\
A_{1 A} & =\frac{42}{49} e^{i \vec{Q} \cdot\left(\frac{2 c_{H}}{3}+z_{1 A}\right) \hat{z}} e^{-\frac{1}{2} Q^{2} \sigma_{1 A}^{2}} \\
A_{\text {Ad-atom }} & =\frac{12}{49} e^{i \vec{Q} \cdot\left(\frac{2 c_{H}}{3}+z_{1 A}+z_{\text {Ad-atom }}\right) \hat{z}} e^{-\frac{1}{2} Q^{2} \sigma_{\text {Ad-atom }}^{2}} .
\end{aligned}
$$

The origin is at the top of the bulk $\operatorname{Si}(111)_{C}, c_{H}$ is the vertical lattice parameter, $z_{j}$ are deviations in the vertical heights from the theoretical position of the $j t h$ layer,

and $e^{-\frac{1}{2} Q^{2} \sigma_{j}^{2}}$ is the Gaussian distribution of electrons where $\sigma_{j}$ is the RMS variation of the $j$ th layer vertical position.

The vertical structure parameters of the Si7x7 surface reconstruction were refined to give slight improved fits, where changes did not exceed $\pm 0.08 \AA$ from the previously determined[88] vertical positions. The Si7x7 layer root-mean-square (RMS) roughness parameters were fixed at $0.1 \AA$.

The total temperature dependent specular scattering amplitudes from the bulk $\mathrm{Si}(111)_{C}$, from Eq. (7.7), with the $7 \times 7$ reconstructed surface, from Eq. (7.10), is

$$
A_{\text {total }}^{\prime}=\left(A_{S i(111)_{C}-S p e c}^{\prime}+A_{7 x 7}^{\prime}\right) V_{s u b}
$$

where $V_{\text {sub }}$ is determined from Eq. (7.9).

\section{Thin Ag Film}

Previous studies[72, 86] by our group have determined the structure of thin $\mathrm{Ag}$ films grown on $\mathrm{Si}(111)_{C} 7 \mathrm{x} 7$ for the same sample used in this study. The structure was 
refined to give slight improved fits. We will use the island height distribution model that was implemented by Chen et al. $[72,86]$ to explain the distribution of $j$-layer Ag islands on $\mathrm{Si}(111)_{C} 7 \times 7$.

The Ag coverage is expressed as

$$
\Theta=p_{w e t} \phi_{w e t}+\sum_{j=1} p_{j} \sum_{n=1}^{j} \phi_{n}
$$

where $p_{w e t}$ and $p_{j}$ are the portions of the surface occupied by the wetting layer and island of height $j$, and $\phi_{w e t}$ and $\phi_{n}$ are the areal densities of the wetting layer and islands, respectively. $\phi_{n}=1$ is for the FCC Ag in the islands. The $p$ values are constrained so that all the surface fractions sum to unity,

$$
1=p_{w e t}+\sum_{j=1}^{\infty} p_{j}
$$

Therefore, using Eq. (7.13), the out-of-plane Ag islands structure factor is determined by

$$
F_{\text {Ag thin }}=f^{A g}(Q)\left(p_{w e t} \phi_{w e t} e^{i Q z_{0}}+\sum_{j=1} p_{j} \sum_{n=1}^{j} \phi_{n} e^{i Q z_{n}}\right)
$$

where $f_{c}^{A g}(Q)$ is the tabulated[64] Ag atomic form factor, $z_{0}$ is the height of the wetting layer above Si7x7 layer-1A, and $z_{n}=z_{0}+d_{1}+d_{2}+\cdots d_{n-1}$. The Ag atomic layer spacing along $\left\langle 111>_{C}\right.$ is $d_{A g}=2.361 \AA$. Taking into account the root-meansquare (RMS) roughness, $e^{-\frac{1}{2} Q^{2} \sigma^{2}}$, of each Ag layer, and the thermal Debye Waller factor for $\mathrm{Ag}, T_{c}^{A g}(Q)$, the temperature dependent specular scattering amplitude of the $\mathrm{Ag}$ islands is

$$
A_{A g \text { thin }}^{\prime}=f^{A g}(Q) T_{c}^{A g}(Q)\left(p_{\text {wet }} \phi_{\text {wet }} e^{i Q z_{0}} e^{-\frac{1}{2} Q^{2} \sigma_{\text {wet }}^{2}}+\sum_{j=1} p_{j} \sum_{n=1}^{j} \phi_{n} e^{i Q z_{n}} e^{-\frac{1}{2} Q^{2} \sigma_{j}^{2}}\right)
$$


where $\sigma_{w e t}$ and $\sigma_{j}$ are the RMS variation of the wetting layer and $j t h$ layer vertical position, respectively.

The total temperature dependent specular scattering amplitude of thin Ag film, from Eq. (7.16), on $\mathrm{Si}(111)_{C} 7 \times 7$, from Eq. (7.12), is expressed as

$$
A_{\text {total }}^{\prime}=\left(\rho_{S i}\left(A_{S i(111)_{C}-S p e c}^{\prime}+A_{7 x 7}^{\prime}\right)+\rho_{A g} A_{A g \text { thin }}^{\prime}\right) V_{\text {sub }}
$$

where $\rho_{S i}$ and $\rho_{A g}$ are the bulk areal densities of $\operatorname{Si}(111)_{C}$ and $\operatorname{Ag}(111)_{C}$, respectively, and the densities are related by $\rho_{A g}=1.763 \rho_{S i}$.

\section{Thick Ag Film}

When Ag films are thick, sensitivity for distinguishing the coverage of $j$-layer islands is lost and therefore the use of the island height model is not practical. We turn to the binomial distribution model, which was discussed elsewhere[41, 87] to model the distribution of $p_{j}$ islands for a thick Ag film. A binomial height distribution of islands with a maximum height of $M$ (only integer values) and an average height of $\bar{n}$ is given as

$$
\frac{1-\left(p e^{i Q d_{A g}}+q\right)^{M}}{1-e^{i Q d_{A g}}}
$$

where $p=\frac{\bar{n}}{M}$ is the probability of occupying a site and the probability of not occupying a site is $q=1-p$. The temperature dependent specular scattering amplitude from a thick Ag film is expressed as[72]

$$
\begin{aligned}
A_{A g \text { thick }}^{\prime}= & f_{c}^{A g}(Q) T_{c}^{A g}(Q)\left[p_{\text {wet }} \phi_{\text {wet }} e^{i Q z_{0}} e^{-\frac{1}{2} Q^{2} \sigma_{\text {wet }}^{2}}\right. \\
& \left.+\left(1-p_{\text {wet }}\right) e^{i Q z_{0}} e^{-\frac{1}{2} Q^{2} \sigma^{2}} \frac{1-\left(p e^{i Q d_{A g}}+q\right)^{M}}{1-e^{i Q d_{A g}}}\right]
\end{aligned}
$$


where $\sigma$ is the RMS roughness of the islands. In comparison to the thin film model, the binomial distribution model simplifies the description of the Ag film - only two parameters, $M$ and $\bar{n}$, are required to explain the height distribution of the thick film.

The total temperature dependent specular scattering amplitude of thick Ag film, from Eq. (7.19), on $\operatorname{Si}(111)_{C} 7 x 7$, Eq. (7.12), is expressed as

$$
A_{\text {total }}^{\prime}=\left(\rho_{S i}\left(A_{S i(111)_{C}-S p e c}^{\prime}+A_{7 x 7}^{\prime}\right)+\rho_{A g} A_{A g-t h i c k}^{\prime}\right) V_{s u b}
$$

\subsubsection{Bare $\operatorname{Si}(111)_{C} 7 \times 7$}

The best-fit of the non-spherical (also shown in Fig. 7.6) and conventional spherical (also seen in Fig. 7.1) charge density distribution models to the X-ray specular reflectivity of bare $\mathrm{Si}(111)_{C} 7 \mathrm{x} 7$ is shown in Fig. $7.7(\mathrm{a})$. A magnified view near the base of the $\operatorname{Si}(222)_{C}$ forbidden reflection is shown in Fig. 7.7(d). Eq. (7.12) was used to calculated specular scattering amplitude for the model curves, where $\operatorname{Im}\left(\gamma^{S i}(\vec{Q})\right)=0$ and $\neq 0$ for the spherical and non-spherical charge density distribution models, respectively. The percent difference between the best-fit curves of the two models is shown in Fig. 7.7(b) and is determined by

$$
\Delta I \%=\frac{\left|I_{\text {non-spherical }}-I_{\text {spherical }}\right|}{I_{\text {spherical }}} \times 100 .
$$

$\Delta I \%$ is an indicator of the sensitivity to the non-spherical charge density distribution. The details about the analysis method are discussed in further below.

Refinement of the structure and charge density distribution parameters were conducted separately, to avoid mutual influence. We start by refining the surface structure. Using the conventional spherical charge density distribution model, the Si7x7

structure parameters were refined from the Robinson values[88]. Influence from the 

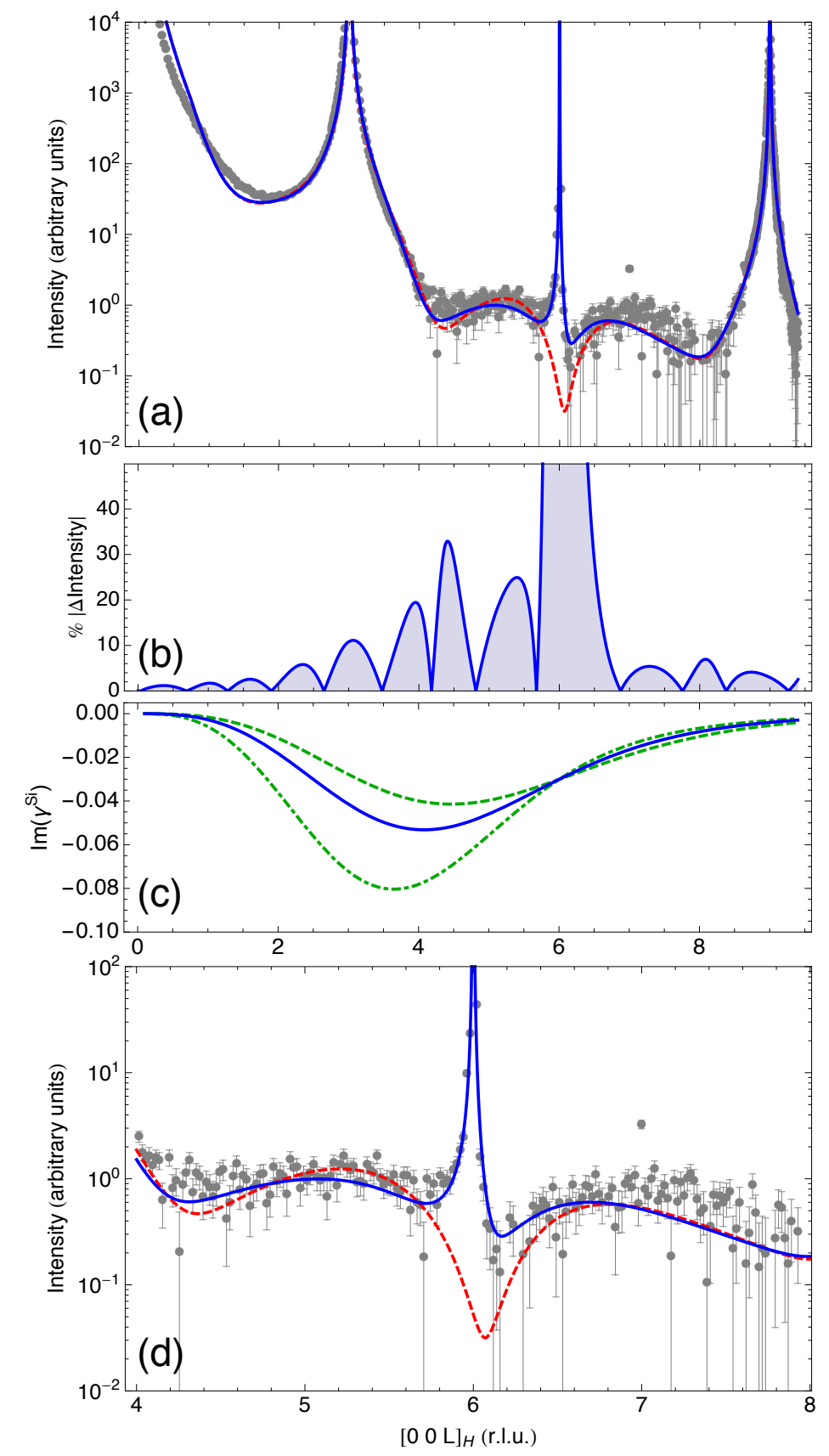

Figure 7.7: (a) Best-fits of the (solid blue curve) non-spherical and (dashed red curve) spherical charge density distribution models to X-ray specular reflectivity data of $\operatorname{Si}(111)_{C} 7 \times 7$. (b) The percent difference in intensity between the spherical and non-spherical charge density distribution models shown in (a). (c) (solid blue curve) Best-fit of $\operatorname{Im}\left(\gamma^{S i}(\vec{Q})\right)$ and for comparison: (dashed and dot-dashed curves) example $\operatorname{Im}\left(\gamma^{S i}(\vec{Q})\right)$ with the same value at $L_{H}=6$, but with different $\alpha$ and $K_{3,22}$ values: $\alpha=1.116$ and $0.744 \AA^{-2}$, and $K_{3,22}=1.790$ and 1.356 for the dashed and dot-dashed curves, respectively. (d) Magnified view of (a) near $\mathrm{Si}(222)_{C}$. 
forbidden Bragg reflection on the surface structure parameter refinement was reduced by temporally omitting the region of data that contains strong interference, between $L_{H}=5.8$ and 6.1, during this procedure. The best-fit of the conventional spherical charge density distribution model from the surface structure refinement is shown in Fig. 7.1 and by the dashed curves in Fig. 7.7(a) and (d).

After the surface structure is refined we can start to determine the bulk charge density distribution, $\gamma^{S i}(\vec{Q})$, while keeping the surface parameters fixed. We start by determining the phase shift that is responsible for the $\mathrm{Si}(222)_{C}$ forbidden reflection, i.e. $\operatorname{Im}\left(\gamma^{S i}\left(L_{H}=6\right)\right)=$ constant, using the non-spherical charge density distribution model. As we discussed above, the phase shift caused by the anisotropic charge density distribution is dependent on the contributions of the asymmetric bonding and the anharmonic thermal vibrations of the core. Due to geometry, anharmonic contributions in Eq. (7.7) are negligible at 300K for the $\mathrm{Si}(222)_{C}$ reflection. Therefore, this reflection is dependent on the details of the asymmetric bonding charge density distribution, Eq. (5.4b), which depends on the radial distribution parameter, $\alpha$, and the scale factor, $K_{3,22}$. However, non-unique combinations of the radial dependence and scale factor result in the same phase shift at the peak, $\operatorname{Im}\left(\gamma^{S i}\left(L_{H}=6\right)\right)$, and therefore the values of $\alpha$ and $K_{3,22}$ cannot be resolved from a single peak. For example, Fig. 7.7(c) shows three $\operatorname{Im}\left(\gamma^{S i}\left[\begin{array}{ll}0 & 0\end{array}\right]_{H}\right)$ curves that have different values of $\alpha$ and $K_{3,22}$ but they share the same value at the $\operatorname{Si}(222)_{C}$ forbidden reflection and therefore are indistinguishable at $\mathrm{L}_{H}=6$. Yet, they have different explanations of the electron density distribution of the covalent bond.

To resolve the electron density distribution of the covalent bonds it is helpful to first determine the contribution of the anisotropic charge density distribution at the forbidden Bragg reflection - we need to determine $\operatorname{Im}\left(\gamma^{S i}\left(L_{H}=6\right)\right)$. Afterwards the $\vec{Q}$ dependence can be resolved. Fortunately, the $\gamma$-formalism consolidates the 
details of the distribution into a single factor that depends on the net anisotropic charge density distribution, see Eq. (5.32b). The phase at the forbidden reflection can be conveniently resolved by determining $\operatorname{Im}\left(\gamma^{S i}\left(L_{H}=6\right)\right)=$ constant. First, the value of $\operatorname{Im}\left(\gamma^{S i}\left(L_{H}=6\right)\right)$ is established based on the quality of fit to the cusp and peak. The cusp side of the peak is used to determine $\operatorname{Im}\left(\gamma^{S i}\left(L_{H}=6\right)\right)=$ constant because the deconstructive interference is the dominant contributor to the scattering in this region and therefore is sensitive to $\operatorname{Im}\left(\gamma^{S i}\left(L_{H}=6\right)\right)$. This behavior is seen in our experimental data in Fig. 7.8. The X-ray specular reflectivity from two different $\operatorname{Si}(111) 7 \times 7$ samples is shown and the differences between the two sets of data is because of slight detailed structural differences between the Si7x7 reconstructed surfaces. Interestingly, in the presence of different surface morphologies the cusp side of the forbidden Bragg reflection remains the same, which indicates that the origin of this feature is from the bulk, rather than the surface, and that the scattering from the bulk is the dominant factor at the cusp. The details of this "characteristic edge" is significantly determined by the phase from the bulk and more specifically the dominant extinction from the diamond double layer structure.

Remarkably, using the "characteristic edge" as a guide, the same anisotropic charge density distribution correction value of, $\operatorname{Im}\left(\gamma^{S i}\left(L_{H}=6\right)\right)=-0.034 \pm 0.005$, is able to explain both samples in Fig. 7.8. This result indicates that $3.4 \%$ of the total atomic form factor at $L_{H}=6$, which is equivalent to 0.204 electrons per atom, is from the anisotropic charge density distribution and that it is dominated by the asymmetric bonding contribution. This value is consistent with the previous work shown in Table 7.1. It is worth mentioning that the previous experimental work was only able to measure the magnitude of the $\mathrm{Si}(222)_{C}$ anisotropic charge density because those measurements were insensitive to the sign. Our work is the first time that both the magnitude and sign of the anisotropic charge density distribution could 


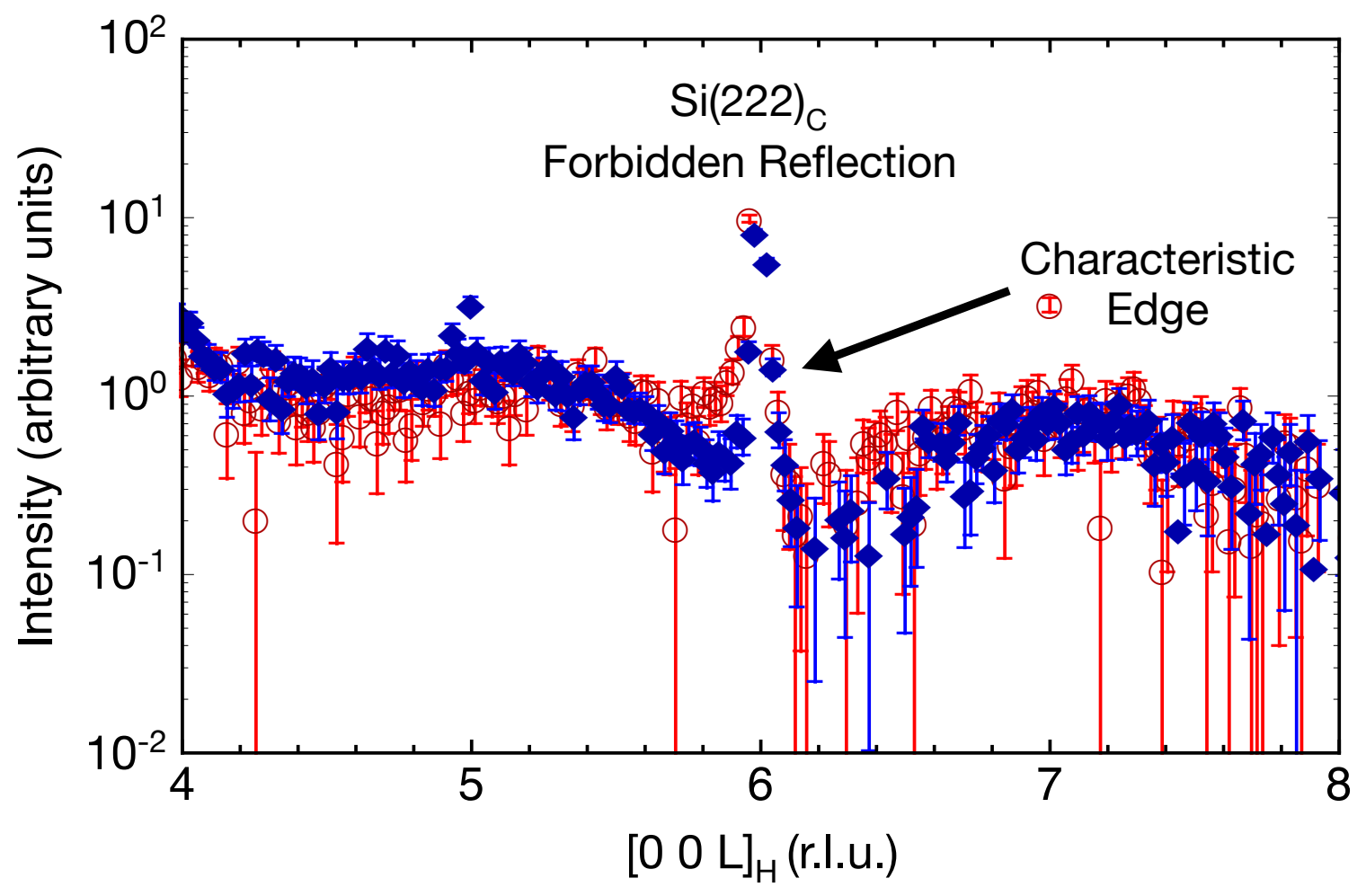

Figure 7.8: Specular X-ray reflectivity data of two different bare $\operatorname{Si}(111)_{C} 7 \times 7$ samples near the $\mathrm{Si}(222)_{C}$ forbidden reflection. Variations in the Si7x7 surface morphology are responsible for the differences between the two sets of data. The characteristic edge of the forbidden reflection is on the $L_{H}>6$ side of the peak, where the shape is independent of the surface morphology. 
Table 7.1: Comparison of structure factor and $\gamma$ values for $\mathrm{Si}(222)_{C}$ at room temperature. The structure factor values are from Alkire et al.[90] Error bars are shown in parenthesis. The error bars from the current work were established by determining the range that the parameter could be varied while maintaining an acceptable fit to the data. The structure factor and $\operatorname{Im}\left(\gamma^{S i}(222)_{C}\right)$ are related by: $F_{S i(222)_{C}}=8 T_{c}^{S i} \bar{f}_{c}^{S i} \operatorname{Im}\left(\gamma^{S i}(222)_{C}\right)$. Note: Two "Current Work" values are shown here and they correspond to the method used for determining the values: *Determined from the data in the proximity of $\operatorname{Si}(222)_{C}$. †Determined from refining $\alpha$ and $K_{3,22}$ with the extended range specular reflectivity.

\begin{tabular}{llll}
\hline & & & \\
Authors & Year & $\mathbf{F}\left(\mathbf{S i}(\mathbf{2 2 2})_{\mathbf{C}}\right)$ & $\mathbf{I m}\left(\gamma^{\mathbf{S i}}(\mathbf{2 2 2})_{\mathbf{C}}\right)$ \\
\hline \hline Hewat, Prager, Stephenson and Wagenfeld & 1969 & 0.88 & -0.0184 \\
Aldred and Hart (model value) & 1973 & $1.35(0.04)$ & $-0.0282(0.0008)$ \\
DeMarco and Weiss & 1965 & $1.44(0.08)$ & $-0.0300(0.0017)$ \\
Current Work†(Extended Range) & $\mathbf{2 0 1 8}$ & $\mathbf{1 . 4 4 ( 0 . 0 4 )}$ & $\mathbf{- 0 . 0 3 0 1 ( \mathbf { 0 . 0 0 0 9 } )}$ \\
Alkire, Yelon, Schneider & 1982 & $1.456(0.008)$ & $-0.0304(0.00017)$ \\
Roberto and Batterman & 1970 & $1.46(0.04)$ & $-0.0305(0.0008)$ \\
Jennings & 1969 & $1.48(0.03)$ & $-0.0309(0.0006)$ \\
Fujimoto (CuK $\left.\alpha_{1}\right)$ & 1974 & $1.48(0.02)$ & $-0.0309(0.0004)$ \\
Schneider, Hansen, and Pattison & 1980 & $1.5(0.05)$ & $-0.0313(0.0010)$ \\
Fujimoto (Pendellösung value) & 1974 & $1.5(0.015)$ & $-0.0313(0.0003)$ \\
Fujimoto (AgK $\left.\alpha_{1}\right)$ & 1974 & $1.51(0.02)$ & $-0.0315(0.0004)$ \\
Colella and Merlini & 1966 & 1.54 & -0.0321 \\
Renninger & 1960 & 1.55 & -0.0323 \\
Current Work* (Si(222) $C$ Reflection) & $\mathbf{2 0 1 8}$ & $\mathbf{1 . 6 3 ( 0 . 2 4 )}$ & $\mathbf{- 0 . 0 3 4}(\mathbf{0 . 0 0 5})$ \\
Fehlmann and Fujimoto (Pendellösung value) & 1975 & $1.65(0.03)$ & $-0.0344(0.0006)$ \\
Cramb & 1970 & $1.76(0.03)$ & $-0.0367(0.0006)$ \\
Göttlicher and Wölfel & 1959 & 1.78 & -0.0371 \\
\hline
\end{tabular}

be experimentally determined using one measurement.

A word of caution before we proceed with refining the parameters that govern the asymmetric bonding distribution: refining $\alpha$ and $K_{3,22}$ simultaneous without determining $\operatorname{Im}\left(\gamma^{S i}\left(L_{H}=6\right)\right)$ is prone to influence from unintended sources, such as the surface structure, and therefore is susceptible to misinterpretation. As seen by the difference in intensities between the spherical and non-spherical charge density distribution models in Fig. 7.7(b), the forbidden reflection and the adjacent cusp are the most sensitive regions along the CTR to the details of the anisotropic charge 
density. Therefore it is prudent to extract and define the phase from the forbidden Bragg reflection first. Further refinement of the charge density distribution is more successful when $\operatorname{Im}\left(\gamma^{S i}\left(L_{H}=6\right)\right)$ at the forbidden reflection is resolved and fixed, because this provides a valuable constraint during the analysis.

We treat the atomic form factor as a continuous function along the CTR, rather than conventionally as a quantity that is only experimentally measured at Bragg peaks. This treatment is analogous to the concept of treating the structure factor as a continuous distribution along the CTR, rather than as an absolute quantity, when a crystal is terminated with an atomically flat surface. [82] The broad influence along the CTR from the few electrons that contribute to the non-spherical charge density distribution is evident in Fig. 7.7(a) and (b). The oscillations in Fig. 7.7(b) are attributed to the Si7x7 structure. The impact on the scattering is not simply contained near the forbidden Bragg reflection but rather is extended broadly along the specular reflectivity rod. Therefore, the CTR can be utilized to determine the structure of the covalent bond in $\mathrm{Si}$.

After determining $\operatorname{Im}\left(\gamma^{S i}\left(L_{H}=6\right)\right)$ we can proceed with refining $\alpha$ and $K_{3,22}$. Seeing as the non-spherical electron density distribution impacts the scattering along the whole CTR and is not simply contained near the forbidden reflection. The extended range specular reflectivity was used to refine $\alpha$ and $K_{3,22}$ from Fehlmann's values[59]. $\operatorname{Im}\left(\gamma^{S i}\left(L_{H}=6\right)\right)$ was kept fixed, which is a valuable constraint that allows $K_{3,22}$ to be dependent on $\alpha . K_{3,22}$ was chosen as the dependent parameter because $\alpha$ dictates the radial distribution and therefore the shape of the charge density distributed along the bond and therefore along the specular reflectivity rod. The refined values of $\alpha$ and $K_{3,22}$ are in Table 7.2. The error bars were established by determining the range that $\alpha$ and $K_{3,22}$ could be varied while maintaining an acceptable fit to the data. As a result $\operatorname{Im}\left(\gamma^{S i}\left(L_{H}=6\right)\right)$ also varied because it is dependent on $\alpha$ and $K_{3,22}$. Therefore, 
Table 7.2: $\quad$ Si charge density distribution parameter values. $\dagger$ In the current work $L_{4,22}$ was not refined, therefore the previously determined value from Fehlmann[59] was used in our models. The error bars in the current work were established by determining the range that $\alpha$ and $K_{3,22}$ could be varied while maintaining an acceptable fit to the data. Fehlmann's values were also in Table 4.1.

\begin{tabular}{l|lll}
\hline & & & \\
& $\alpha\left(\AA^{-2}\right)$ & $K_{3,22}$ & $L_{4,22}$ \\
\hline \hline Current Work & $0.93 \pm 0.02$ & $1.505 \pm 0.0025$ & $-0.206 \pm 0.021 \dagger$ \\
Fehlmann[59] & $0.970 \pm 0.005$ & $1.382 \pm 0.020$ & $-0.206 \pm 0.021$ \\
Dawson[54] & 0.88 & 1.11 & -0.32 \\
\hline
\end{tabular}

based on the refined values of $\alpha$ and $K_{3,22}, \operatorname{Im}\left(\gamma^{S i}\left(L_{H}=6\right)\right)$ is $-0.0301 \pm 0.0009$, which agrees with the previously value obtained from fitting the region near the $\operatorname{Si}(222)_{C}$ peak. This result also agrees well with previous studies, as seen in Table 7.1. The significant reduction in the $\operatorname{Im}\left(\gamma^{S i}\left(L_{H}=6\right)\right)$ error bar is because $\alpha$ and $K_{3,22}$ impacts a broad range of the specular reflectivity, whereas the previously obtained result of $\operatorname{Im}\left(\gamma^{S i}\left(L_{H}=6\right)\right)=-0.034 \pm 0.005$ was determined from a limited range near the forbidden Bragg reflection.

Figure 7.9 shows the non-spherical component of the real-space Si electron density distribution. Experimentally determined values of $\alpha$ and $K_{3,22}$, along with the previously determined value of $L_{4,22}$ from Table 4.1, were used to render the density plot. The shape of the covalent bonds shown here resemble that of an American football or rugby ball, where the cross-sectional slice perpendicular to the bonding axis is circular and has a radial dependence outward from the bonding axis. As expected, we find that the density is greatest between the atoms. The shape of the electron density distribution is consistent with previous results[51, 54].

The non-spherical charge density distribution is a striking improvement from the conventional model. It is able to explain the experimental data of the forbidden reflection peak as well as the interference along the reflectivity rod. strikingly improves 


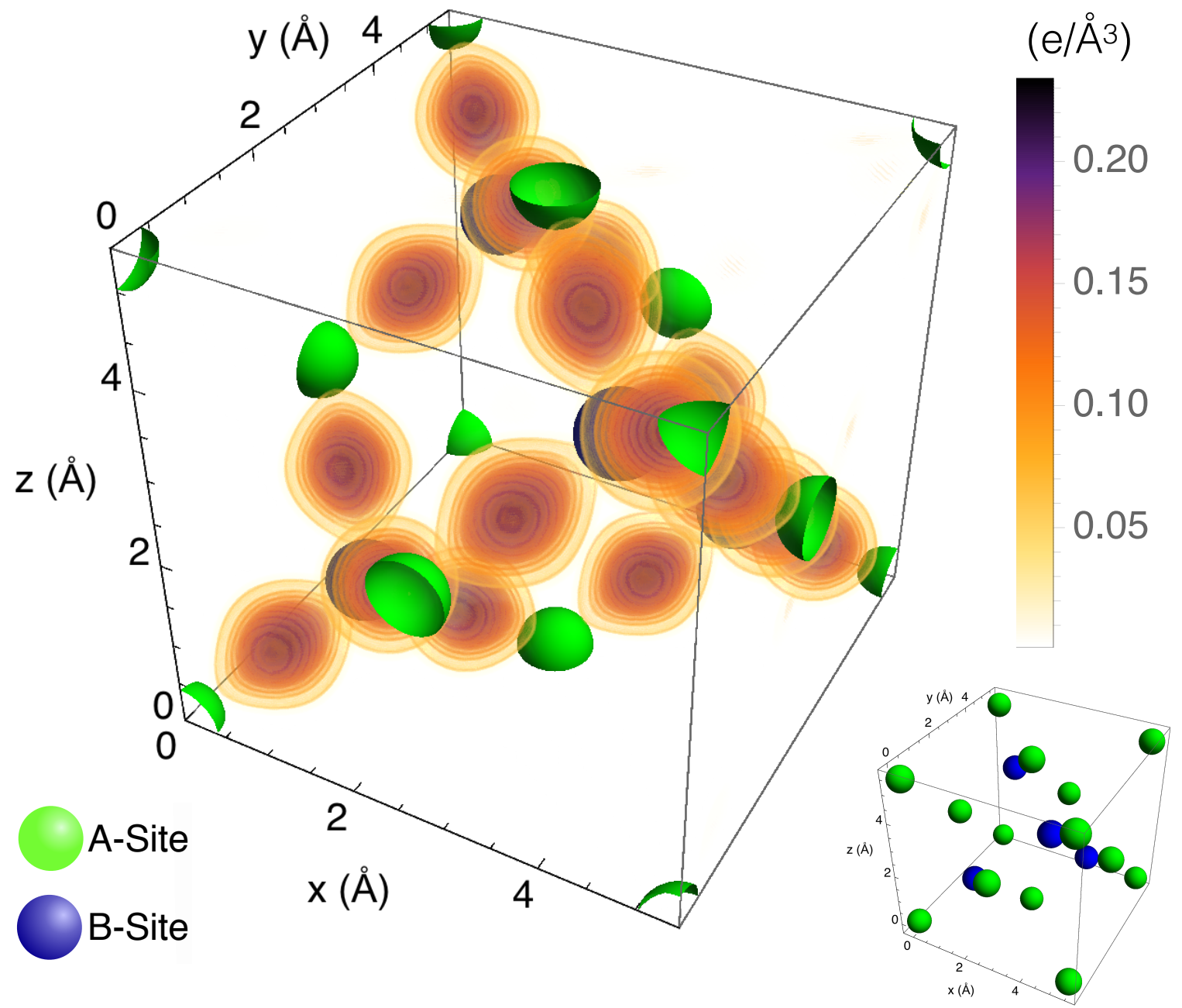

Figure 7.9: Non-spherical component of the real-space Si electron density distribution determined from the X-ray specular reflectivity in Fig. 7.7. For visual clarity, green and blue spherical shells are overlaid at the A- and B-basis sites, respectively. (Bottom right) Schematic figure of the Si cubic cell without the electron density distribution, to help guide the eye. The density distribution was rendered using experimentally determined values of $\alpha$ and $K_{3,22}$, and previously the determined value of $L_{4,22}$, seen in Table 4.1. For visual clarity the density plot is clipped at $0.001 \mathrm{e} / \AA^{3}$. The shape of the covalent bonds shown here resemble that of an American football or rugby ball, where the cross-section perpendicular to the bonding axis is circular. The rings in the covalent bonds are rendering artifacts. 
the models ability to explain This is the first time that the radial distribution, amplitude, and direction of the asymmetric bond charge density distribution have all been determined with a single X-ray measurement.

\subsubsection{Thin Ag Film on $\operatorname{Si}(111)_{C} 7 \times 7$}

The bulk anisotropic charge density can also be determined for surfaces that include thin and thick films. Figure 7.10(a) shows the best-fit curve of the non-spherical charge density distribution model, from Eq. (7.17), to the specular reflectivity data from a thin $\mathrm{Ag}$ film on $\mathrm{Si}(111)_{C} 7 \mathrm{x} 7$ along with a best-fit curve of the conventional charge density distribution model $\left(\gamma^{S i}(\vec{Q})=1\right)$ for comparison. Previously determined[86] surface morphology parameters, including Ag island coverage values, were used to model the thin Ag film sample. Scattering from the Ag was found to be dominant between the $\mathrm{Si}(111)_{C}$ and $(333)_{C}$ Bragg peaks, where the oscillations between $\mathrm{L}_{H}=3$ and 9 correspond to a surface predominantly covered by 3 monolayer (ML) tall $\mathrm{Ag}$ islands and a total of $\mathrm{MLL}$. The bulk Si charge density distribution is inde-

pendent of the surface therefore, it is appropriate to use the refined $\gamma^{S i}(\vec{Q})$ from the bare $\operatorname{Si}(111)_{C} 7 \times 7$ sample. The refined values of $\alpha$ and $K_{3,22}$ can be found in Table 7.2. Compared with the conventional model, the non-spherical charge density distribution model shows noticeable improved agreement with the data around the $\mathrm{Si}(222)_{C}$ forbidden Bragg reflection, as seen in the magnified view in Fig. 7.10(c) between $\mathrm{L}_{H}=6$ and 6.3, while also maintaining its agreement with the data elsewhere.

In the presence of amplitude contributions from the $\mathrm{Ag}$ film, the $\mathrm{Si}(222)_{C}$ forbidden Bragg reflection and the adjacent cusp experience a symmetry flip. The cusp on the bare $\operatorname{Si}(111)_{C} 7 \times 7$ samples resides on the side of the peak away from the origin, as seen in Fig. 7.7(c). Whereas with the addition of the Ag film the cusp resides on 

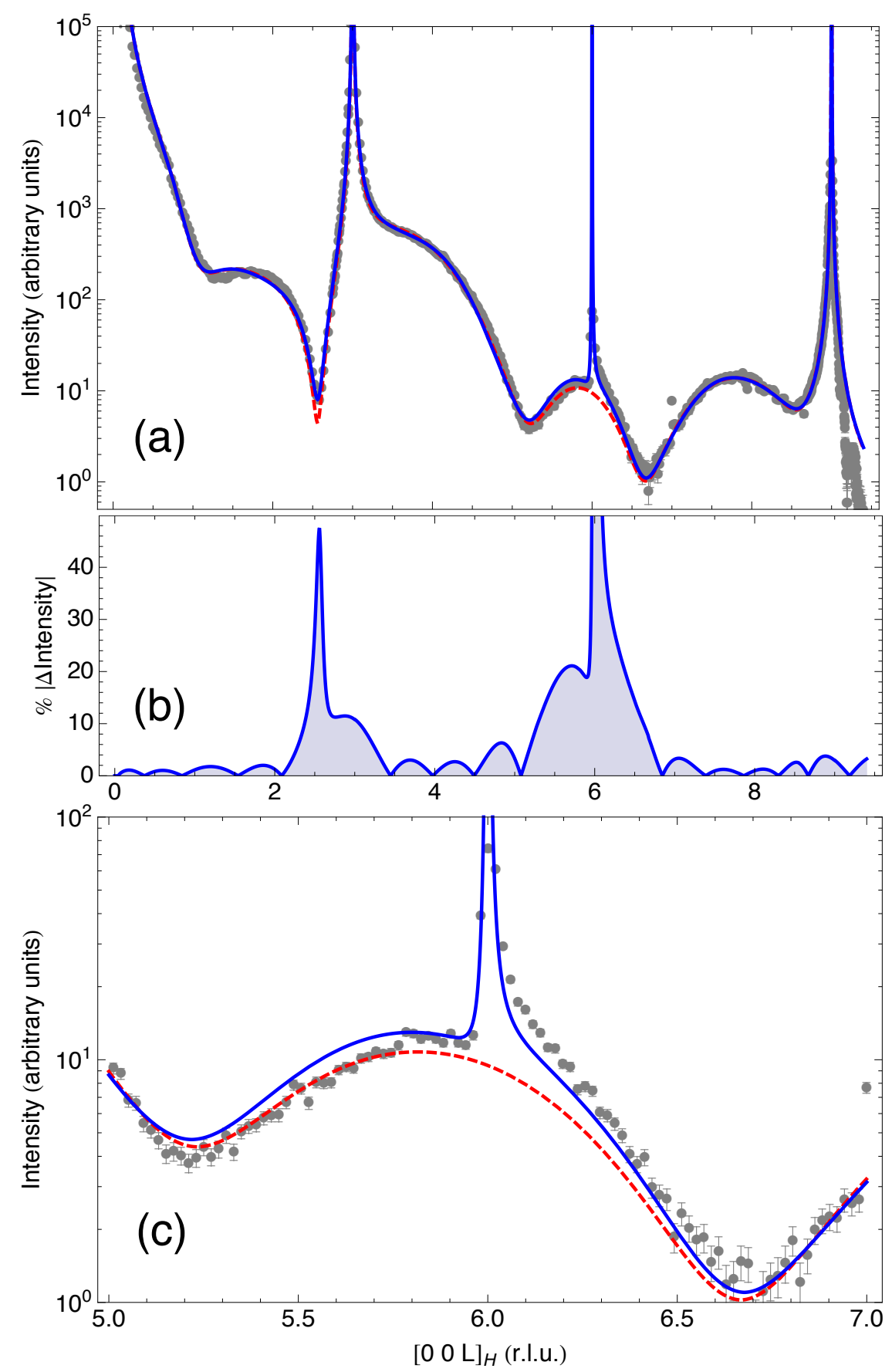

Figure 7.10: (a) and (c) best-fit of the (solid blue curve) non-spherical and (dashed red curve) spherical charge density distribution models to X-ray specular reflectivity data of thin $\mathrm{Ag}$ film on $\mathrm{Si}(111)_{C} 7 \times 7$. (c) is a magnified view of the extended range reflectivity shown in (a), near the base of the $\operatorname{Si}(222)_{C}$ forbidden reflection. (b) shows the percent difference in intensity between the spherical and non-spherical charge density distribution models shown in (a). 
the side towards the origin. The symmetry flip is explained by the additional phase contribution from the Ag film. Notably, our model, which uses $\gamma^{S i}(\vec{Q})$ determined from the bare $\mathrm{Si}(111)_{C} 7 \times 7$ sample, also agrees well with the samples that include $\mathrm{Ag}$ films and explains the symmetry flip without any modifications.

The percent difference in intensity between the non-spherical charge density distribution model and the conventional model is shown in Fig. 7.10(b). It can be seen that the Ag film dampens the sensitivity to the non-spherical charge density distribution throughout much of the specular reflectivity rod. This is the result of the Ag film amplitude suppressing the relatively weak contribution from the bulk $\mathrm{Si}$ anisotropic charge density distribution. However, there is a significant change in intensity in the region around $L_{H} \approx 2.5$. Compared with the bare $\mathrm{Si}(111)_{C} 7 \times 7$ sample (see Fig. 7.7(b)), the difference in intensity in this region has increased appreciably for the sample with thin $\mathrm{Ag}$ film. The depth of the cusp near $L_{H} \approx 2.5$ is sensitive to $\operatorname{Im}\left(\gamma^{S i}(\vec{Q})\right)$ - four times more sensitive than at the $\operatorname{Si}(111)_{C}$ Bragg peak. This effect is noticeable in Fig. 7.10(a), the conventional model over shoots the data, extending too low, while the non-spherical distribution model shows better agreement with the data. Therefore, the region near $L_{H} \approx 2.5$ and around the forbidden reflection, together, are sensitive to the details of the asymmetric bonding distribution and can be used to refine the non-spherical charge density distribution parameters.

The enhancement in sensitivity at the $L_{H} \approx 2.5$ cusp prior to the $\operatorname{Si}(111)_{C}$ peak can be explained by the destructive interference between the $\operatorname{Si}(111)_{C}$ peak and the Ag film. The two amplitudes are competing at the cusp, which reduces the competition for the scattering contribution from the bulk covalent bonds. The amplitude cancellation is analogous to the interference at the $\mathrm{Si}(222)_{C}$ reflection, where the strong contributions from the core electrons cancel out and leaves the anisotropic charge density distribution. In the case of the $L_{H} \approx 2.5$ cusp the core electrons from 
the bulk Si are interfering with the Ag film and revealing the bulk Si covalent bonds.

The interference between the $\mathrm{Si}(111)_{C}$ Bragg peak and the Ag film occurs at a fortunate location along $[00 L]_{H}$, because the value of $\operatorname{Im}\left(\gamma^{S i}(\vec{Q})\right)$ is sensitive to $\alpha$ and $K_{3,22}$ in the region near $L_{H} \approx 2.5$. Figure 7.11(a) and (b) show magnified views of the cusp near $L_{H} \approx 2.5$ and the $\mathrm{Si}(222)_{C}$ forbidden Bragg reflection, respectively, along with three model curves. The solid curve is the best-fit to the non-spherical charge density distribution model, the dashed and dot-dashed curve uses different $\alpha$ and $K_{3,22}$ values in the same model (values are in the figure caption), and as a reference the dotted is the best-fit to the conventional spherical charge density distribution model. The corresponding curves of $\operatorname{Im}\left(\gamma^{S i}(\vec{Q})\right)$ with the best-fit and comparison values for $\alpha$ and $K_{3,22}$ are shown in Fig. 7.11(c). The three non-spherical charge density distribution models share the same $\operatorname{Im}\left(\gamma^{S i}\left(L_{H}=6\right)\right)$ and therefore they are indistinguishable near the forbidden Bragg reflection in Fig. 7.11(b). The difference between the three $\operatorname{Im}\left(\gamma^{S i}(\vec{Q})\right)$ curves near $L_{H} \approx 2.5$ is noticeably greater than near in the region between $L_{H}=5$ and 7 . The difference between the two $\operatorname{Im}\left(\gamma^{S i}(\vec{Q})\right)$ curves is expressed in the corresponding specular reflectivity model curves in Fig. 7.11(a). The difference in intensity between the best-fit and comparison curves near the $L_{H} \approx 2.5$ cusp is noticeable. Although, elsewhere along the specular reflectivity the three model curves are indistinguishable. These results indicate that sensitivity to the bulk Si electron density distribution in this sample is limited to two regions along the specular reflection, near the forbidden Bragg reflection and near the $L_{H} \approx 2.5$ cusp.

The non-spherical charge density distribution model curves in Fig. 7.11(a) and (b) agree well with the data and in comparison to the spherical charge density distribution model are significant improvements. Though there are a few critical differences between the non-spherical charge density curves. The dashed curve over extended the $L_{H} \approx 2.5$ cusp in Fig. 7.11(a), while the dot-dashed curve under extends, while 

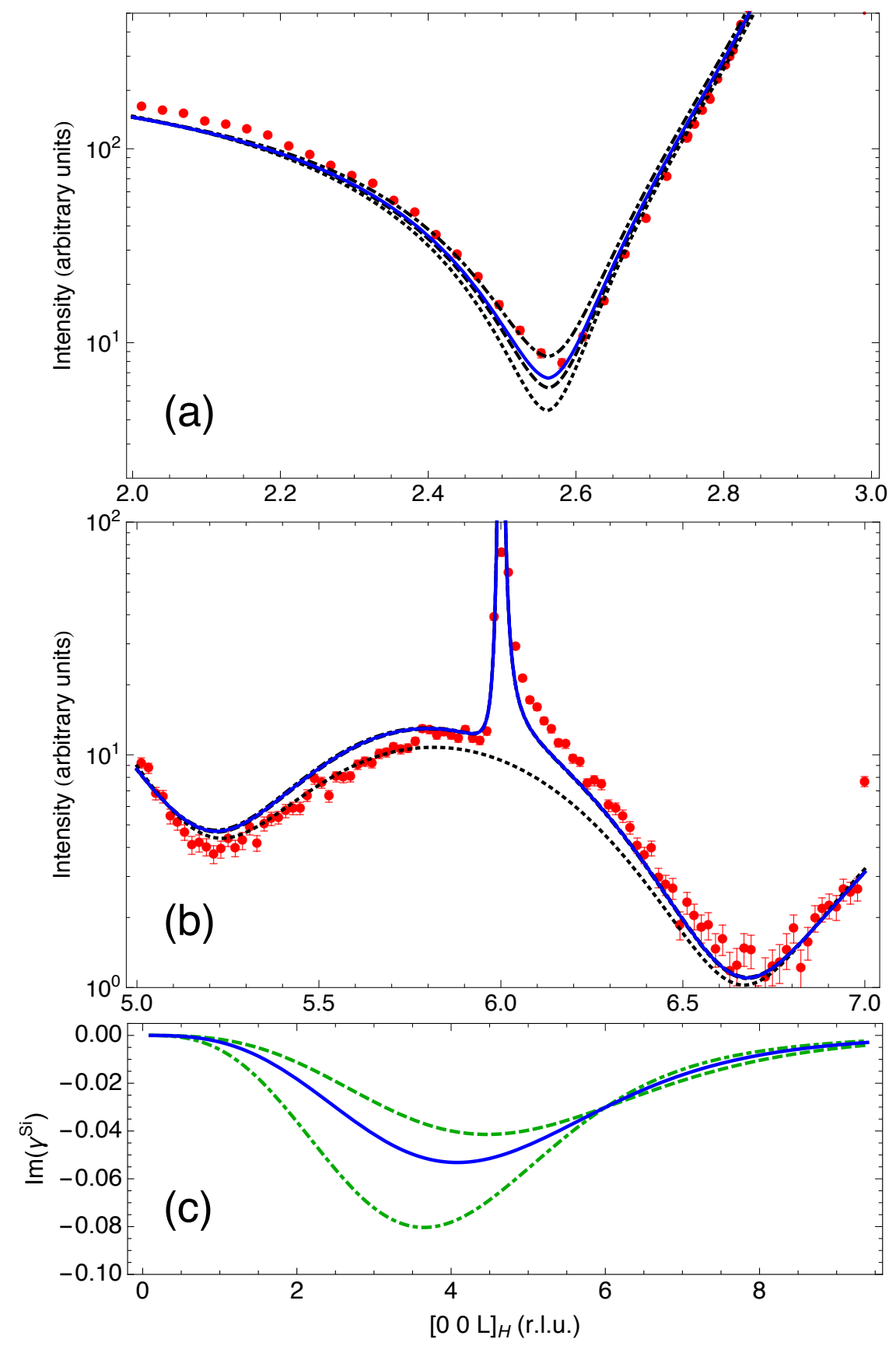

Figure 7.11: (a) and (b) are magnified views of the X-ray specular reflectivity data of thin $\mathrm{Ag}$ film on $\operatorname{Si}(111)_{C} 7 \times 7$. (c) $\operatorname{Im}\left(\gamma^{S i}(\vec{Q})\right)$ Non-spherical charge density distribution model using: (solid blue curve) best-fit values, (dashed curve) $\alpha=1.116 \AA^{-2}$ and $K_{3,22}=1.790$, and (dot-dashed curve) $\alpha=0.744 \AA^{-2}$ and $K_{3,22}=1.356$. (dotted curve) Spherical charge density distribution model for comparison. The best-fit curves and data are identical to that shown in Fig. 7.10. 
the best-fit curve is located in between and slightly over extending. The range in the specular reflectivity curves near the $L_{H} \approx 2.5$ cusp indicates that this region is sensitive to the details of the electron density distribution. It is notable that we are sensitive to the structure of the covalent bonds at angles below the $\operatorname{Si}(111)_{C}$

Bragg peak. Particularly as seen by the $\operatorname{Im}\left(\gamma^{S i}(\vec{Q})\right)$ curves shown in Fig. 7.11(c), the structure of the covalent bond varies considerably in this region.

It is worth discussing the shape of the interference between the forbidden Bragg reflection and the $\mathrm{Ag}$ film oscillations. Figure 7.12 shows the X-ray specular reflectivity data of thin $\mathrm{Ag}$ film on $\mathrm{Si}(111)_{C} 7 \mathrm{x} 7$ with non-spherical charge density distribution model curves using (solid curve) the best-fit values of $\alpha$ and $K_{3,22}$ and (dashed) Dawson's[54] values for $\mathrm{Si}$, where $\alpha=0.88 \AA^{-2}$ and $K_{3,22}=1.11$. The two curves are indistinguishable elsewhere along the specular reflectivity rod. The shape of the Dawson's reflectivity curve, on the lower angle side of the forbidden Bragg reflection, agrees with the data better than our best-fit curve. However, the best-fit curve agrees better with the data on the higher angle side of the forbidden reflection. The shape of the forbidden reflection tail on the higher angle side cannot be explained by changes in the Ag island height distribution. Whereas the intensity of the low angle side can be manipulated by refining the details of the Ag film. We are confident that our bestfit values are in better agreement with the data than the Dawson's values because the best-fit curve corresponds better with the high angle side tail of the forbidden reflection.

\subsubsection{Thick Ag Film on $\mathrm{Si}(111)_{C} 7 \times 7$}

Figure 7.13(a) shows the best-fit curve of the non-spherical charge density distribution model, from Eq. (7.20), to the specular reflectivity data from a thick Ag 


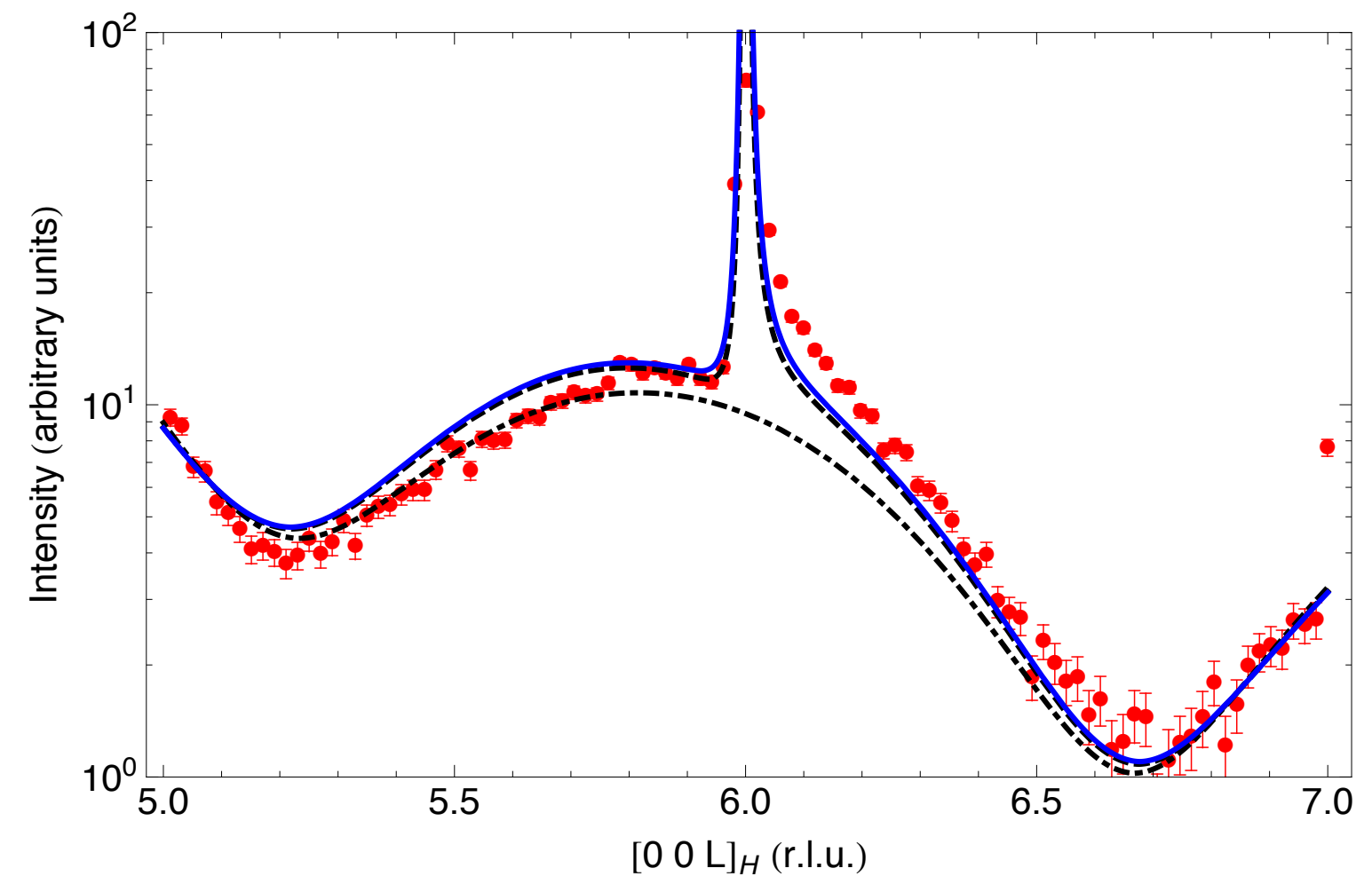

Figure 7.12: Magnified view of the X-ray specular reflectivity data of thin Ag film on $\mathrm{Si}(111)_{C} 7 \times 7$. Non-spherical charge density distribution model using: (solid blue curve) best-fit values and (dashed curve) Dawson's[54] values, $\alpha=0.88 \AA^{-2}$ and $K_{3,22}=1.11$. (dot-dashed curve) Spherical charge density distribution model for comparison. 
film on $\operatorname{Si}(111)_{C} 7 \times 7$ along with a best-fit curve of the conventional spherical charge density distribution model $\left(\gamma^{S i}(\vec{Q})=1\right)$ for comparison. The $\operatorname{Ag}(111)_{C}$ and $(222)_{C}$ Bragg peaks are located near $L_{H}=4$ and 8 , respectively, and the subsidiary oscillations are interference fringes from $32 \mathrm{ML}$ of $\mathrm{Ag}$. In the presence of thick $\mathrm{Ag}$ film the reflectivity is less sensitive to small deviations in the positions of the surface Si7x7 atoms, therefore these parameters were fixed at previously determined values[88]. Initially, the Ag structure parameters were refined by fitting the conventional spherical charge density distribution model to an abridged data set that omitted the region of data around the forbidden reflection. The refined Ag structure parameters were then used in the non-spherical charge density distribution model along with $\gamma^{S i}\left([00 L]_{H}\right)$ determined from the bare $\operatorname{Si}(111)_{C} 7 \times 7$ sample. The refined values of $\alpha$ and $K_{3,22}$ can be found in Table 7.2. The change in intensity between the two fit curves is seen in Fig. 7.13(b). The non-spherical charge density distribution model improves the quality of fit around the forbidden reflection, as seen in Fig. 7.13(c), while maintaining good agreement with the data without further refinement of the non-spherical charge density distribution correction.

Interestingly the thick $\mathrm{Ag}$ film aids in measuring the interference between the forbidden reflection and the surface waves. The thick Ag film enhances the intensity of the scattering between the $\mathrm{Si}(111)_{C}$ and $\mathrm{Si}(333)_{C}$ Bragg peaks. Over an order of magnitude increase in intensity from the bare $\mathrm{Si}(111)_{C} 7 \times 7$ sample is seen in Fig. 7.6(b). The greater electron density of $\mathrm{Ag}$ is largely responsible for the intensity gain. An estimate based on the atomic number indicates an increase by a factor of $11.2=\frac{Z_{A g}{ }^{2}}{Z_{S i}{ }^{2}}$, which is consistent with the enhancement seen in Fig. 7.6(b). The roughness from additional Ag layers dampen out the interference fringes between the Ag Bragg peaks, which results in an amplified and unvarying backdrop for the forbidden Bragg reflection to interfere with the surface waves. Furthermore, the thick Ag film reduces 

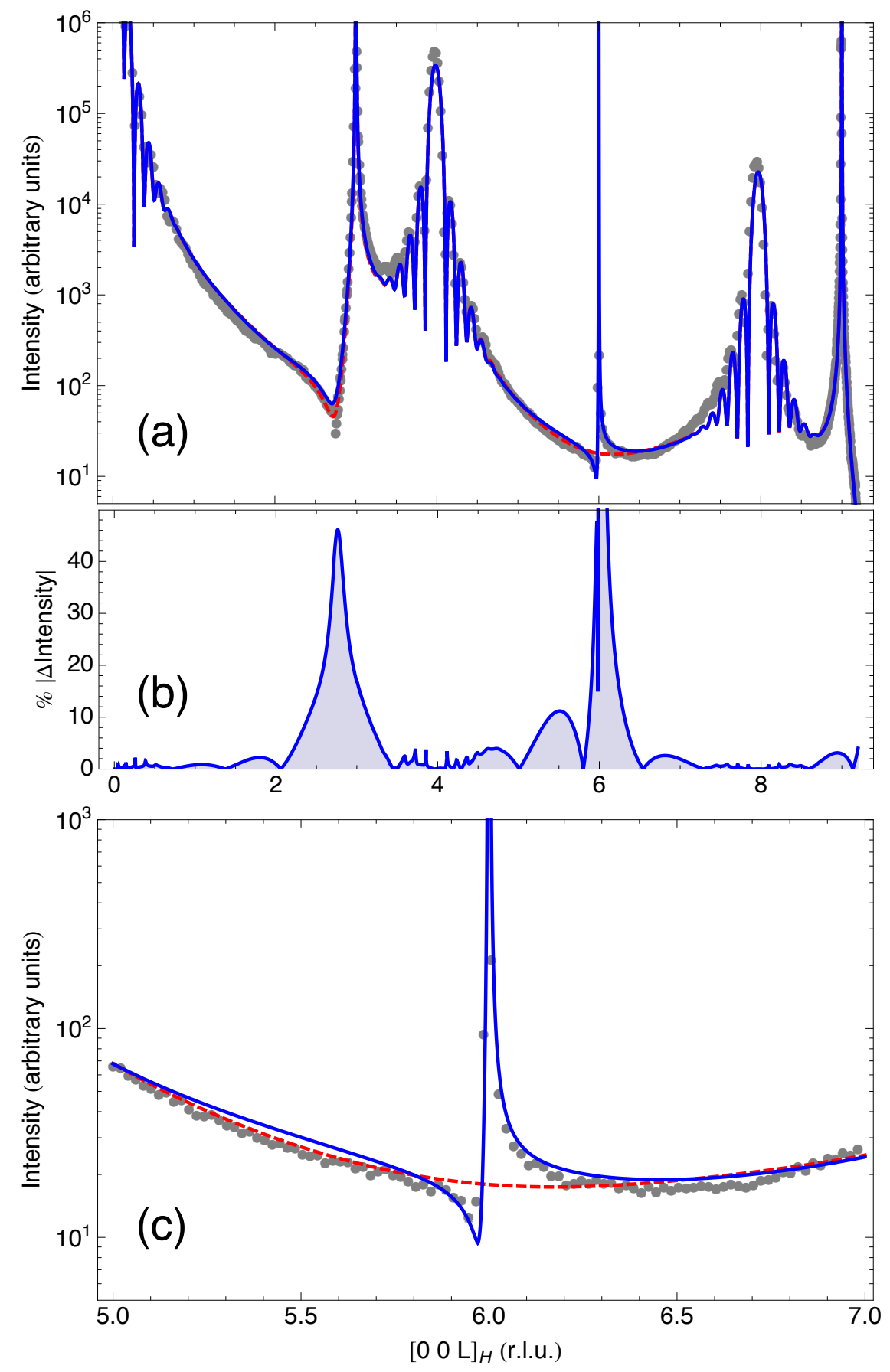

Figure 7.13: (a) and (c) best-fit of the (solid blue curve) non-spherical and (dashed red curve) spherical charge density distribution models to X-ray specular reflectivity data of thick $\mathrm{Ag}$ film on $\mathrm{Si}(111)_{C} 7 \mathrm{x} 7$. (c) is a magnified view of the extended range reflectivity shown in (a), near the base of the $\mathrm{Si}(222)_{C}$ forbidden reflection. (b) shows the percent difference in intensity between the spherical and non-spherical charge density distribution models shown in (a). 
the influence on the scattering from the Si surface, thereby trimming down the main scattering contributions to the bulk $\mathrm{Si}$ and the thick Ag film. The thicker film also dampens the contribution from the bulk Si non-spherical charge density distribution, and this change is noticeable between the percent change in intensities in Fig. 7.10(b) and Fig. 7.13(b). Remarkably, as a result, the thick Ag film enhances the measurement and refinement of $\operatorname{Im}\left(\gamma^{S i}\left(L_{H}=6\right)\right)$ by amplifying the amplitude scattered from the film that can interfere with the forbidden reflection. In addition, the featureless scattering in between the Ag Bragg peaks suppresses the sensitivity to the surface morphology in the region near the forbidden reflection peak.

A reduction in the $\operatorname{Im}\left(\gamma^{S i}\left(L_{H}=6\right)\right)$ error bars is an indicator of the scattering enhancement near the forbidden reflection. The error bars were established by determining the range that $\operatorname{Im}\left(\gamma^{S i}\left(L_{H}=6\right)\right)=$ constant could be varied while maintaining an acceptable fit to the data. $\operatorname{Im}\left(\gamma^{S i}\left(L_{H}=6\right)\right)=$ constant determined from the thick Ag film sample is $-0.033 \pm 0.003$. By comparison to the value determined from the bare $\operatorname{Si}(111)_{C} 7 \times 7$ sample, $-0.034 \pm 0.005$, is notable and reflects the improved conditions for establishing $\operatorname{Im}\left(\gamma^{S i}\left(L_{H}=6\right)\right)=$ constant.

\subsection{Discussion}

We have implemented the CTR technique — conventionally used to study surface structures - in a novel way by using the surface to examine the bulk charge density distribution. Our results provide new insight into the interaction of the nonspherical charge density distribution with CTRs. Our study has demonstrated that

with special care the radial distribution of the asymmetric bonding charge of Si can be obtained from X-ray specular reflectivity. With this one-dimensional measurement, the valence electron density distribution in diamond crystal structures can be 
determined in three-dimensions by exploiting its crystal symmetry. Furthermore, our study has also demonstrated that the anisotropic charge density distribution can be extracted from samples that have thin and thick Ag films, which shows that $\gamma(\vec{Q})$ does not depend on the surface morphology, as long as the surface causes slow intensity variations near the forbidden Bragg reflection.

The most striking result from this study is the discovery of novel interference between the surface and bulk waves that allows the non-spherical charge density distribution in certain crystal structures to be experimentally determined. The new capability arises from two effects:

1. interference between the bulk forbidden Bragg reflection and the CTR; and

2. interference between the non-spherical charge density distribution and the extended range CTR.

Of the two, the former is the stronger scattering effect and the interaction between the bulk and surface waves allows us to extract both the amplitude and the sign of the phase from the forbidden reflection, as we have demonstrated with the $\operatorname{Si}(222)_{C}$ reflection. The latter effect arises from the projection of the continuous non-spherical charge density distribution on to the CTR, which allows us to extract additional information on the radial density distribution of the covalent bond in Si. These novel interference effects allow us to expand the capabilities of X-ray CTR techniques as well as improves ability to determine the crystal surface structure.

By comparison, diffraction techniques are insensitive to the phase of the scattered wave and therefore loses the sign. Previous diffraction studies relied on a combination of temperature dependent X-ray and complimentary neutron diffraction measurements to infer the phase information of the forbidden Bragg reflections. As we discussed in Chapter 5, these studies were challenging experimentally. For example, 
these experiments relied on accurate scaling of the X-ray (probes the core and valence electrons) and neutron (measures the nuclear cross-section) measurements to extract the valence electron density contribution.

In contrast, our discovery allows us to determine the valence electron density distribution in diamond crystal structures from a single CTR measurement. The destructive interference between the bulk forbidden Bragg reflection and the CTR from the surface is highly sensitive to the sign of the phase from the bulk non-spherical charge density distribution. Interestingly, a "characteristic edge," which resides on the cusp side of the forbidden Bragg reflection (see Fig. 7.8), is independent of the details of the surface structure and therefore is helpful for determining the contribution of anisotropic charge density distribution at the forbidden reflection. Even in the presence of Ag a "characteristic edge" remains constant on the cusp side of the peak throughout all of the samples with Ag films. On the samples with Ag films we used the $\operatorname{Im}(\gamma(\vec{Q}))$ determined from the bare $\operatorname{Si}(111)_{C} 7 \mathrm{x} 7$ sample, which provided an impressively good agreement between the model curve and the "characteristic edge" - indicating that this feature originates from the bulk Si structure. We found that our experimentally determined value of the anisotropic charge density distribution correction for the $\operatorname{Si}(222)_{C}$ forbidden reflection, $\operatorname{Im}\left(\gamma^{S i}\left(L_{H}=6\right)\right)=-0.033 \pm 0.003$, was consistent across all our samples and, as seen in Table 7.1, is in agreement with previous results.

Determining the value of $\operatorname{Im}\left(\gamma^{S i}\left(L_{H}=6\right)\right)$ at the forbidden Bragg reflection allowed us to determine the distribution of the covalent bond density distribution along the bonding direction. This could be achieved because the impact of the anharmonic thermal vibrations and the centrosymmetric charge density distribution along the $\langle 111\rangle_{C}$ is negligible between $\operatorname{Si}(000)_{C}$ and $\operatorname{Si}(333)_{C}$ due to geometry. By constraining $\operatorname{Im}\left(\gamma^{S i}\left(L_{H}=6\right)\right)$ at the $\operatorname{Si}(222)_{C}$ forbidden Bragg reflection with the fit value, 
further details about the charge density distribution can be obtained from the bare $\mathrm{Si}(111)_{C} 7 \mathrm{x} 7$ specular reflectivity data. Specifically, the radial dependence, $\alpha$, and scale factor, $K_{3,22}$, parameters could be determined. This is possible, first, because the non-spherical charge density distribution is not constant nor a delta-function in real-space and therefore it is neither in reciprocal-space. Rather the non-spherical charge is distributed in real-space (see Fig. 4.2) and in reciprocal space (see Fig. 5.3). This reason has permitted diffraction techniques to extract charge density distribution information from forbidden and allowed Bragg reflections. Secondly, the scattering from the Si7x7 surface structure along the CTR is weak enough that it does not overwhelm the contribution from the bulk non-spherical charge density distribution. Lastly but not least, the ability to confidently determine $\operatorname{Im}(\gamma(\vec{Q}))$ at a forbidden Bragg reflection greatly aids in determining the asymmetric bonding charge density distribution parameters, $\alpha$ and $K_{3,22}$, from the CTR. The forbidden Bragg reflection is highly dependent on the anisotropic charge density distribution and is independent of the details of the surface structure. Whereas the scattering from the bulk non-spherical charge density distribution competes with the waves from the surface. Therefore by establishing the value of $\operatorname{Im}\left(\gamma^{S i}\left(L_{H}=6\right)\right)$ at the $\operatorname{Si}(222)_{C}$ forbidden Bragg reflection allowed us to set a valuable constraint that permitted us to refine the values of $\alpha$ and $K_{3,22}$ from fitting the specular reflectivity. This technique can also be applied to in-plane CTR geometries. Depending on the geometry, the contributions of the core centrosymmetric charge deformation can also be examined.

Lets take a moment to put our method of determining the asymmetric bonding charge density distribution into perspective. Diffraction techniques rely on the integrated intensity of the Bragg peaks along the $\langle 111\rangle_{C}$ direction. It can be seen from the $\operatorname{Im}(\gamma(\vec{Q}))$ curve in Fig. 5.6 the asymmetric bonding charge density distribution is limited to a region of reciprocal space where $\vec{Q}$ is small. Along the $\langle 111\rangle_{C}$ direction 
the charge density distribution is extended the furthest and has the greatest density along the specular reflectivity rod, $[00 L]_{H}$. This region is more confined along other directions. Therefore diffraction techniques is limited to three Bragg peaks, $(111)_{C}$, $(222)_{C}$, and $(333)_{C}$, to measure and determine the asymmetric bonding charge density distribution. It can be seen from the percent difference in intensity between the spherical and non-spherical charge density distribution models in Fig. 7.7 the contribution of the asymmetric bonding charge density distribution at the $\mathrm{Si}(111)_{C}$ and $\mathrm{Si}(333)_{C}$ reflections is quite small. The difference in intensity at the Bragg peaks due to the asymmetric bonding charge is less than $12 \%$ and $5 \%$, respectively. In contrast, the asymmetric bonding charge density distribution is spread throughout the CTR, therefore the distribution can be determined from the whole rod. Using the CTR to extract charge density distribution information not only increases the sampling of the distribution but is also has the potential to reveal new information between that is contained between the Bragg peaks - a noticeable improvement from a finite number of Bragg peaks.

Furthermore, it is notable that by determining $\alpha$ and $K_{3,22}$ - extracted from the one-dimensional specular X-ray reflectivity measurement - we are able to reconstruct the three-dimensional bonding charge density distribution, as seen in Fig. 7.9, by exploiting the crystal symmetry. This task would not have been possible to perform accurately without also attaining both the sign and amplitude of the anisotropic charge density at the forbidden reflection.

Interestingly, our results show an enhancement in the scattering region near the forbidden reflection by the presence of a Ag film. We found that with a thick Ag film the intensity of the CTR near the $\mathrm{Si}(222)_{C}$ forbidden reflection increases by over one order of magnitude from the bare $\mathrm{Si}(111)_{C} 7 \mathrm{x} 7$ sample, as seen in Fig. 7.6(b). The increased intensity aids in the ability to experimentally measure the interference at 


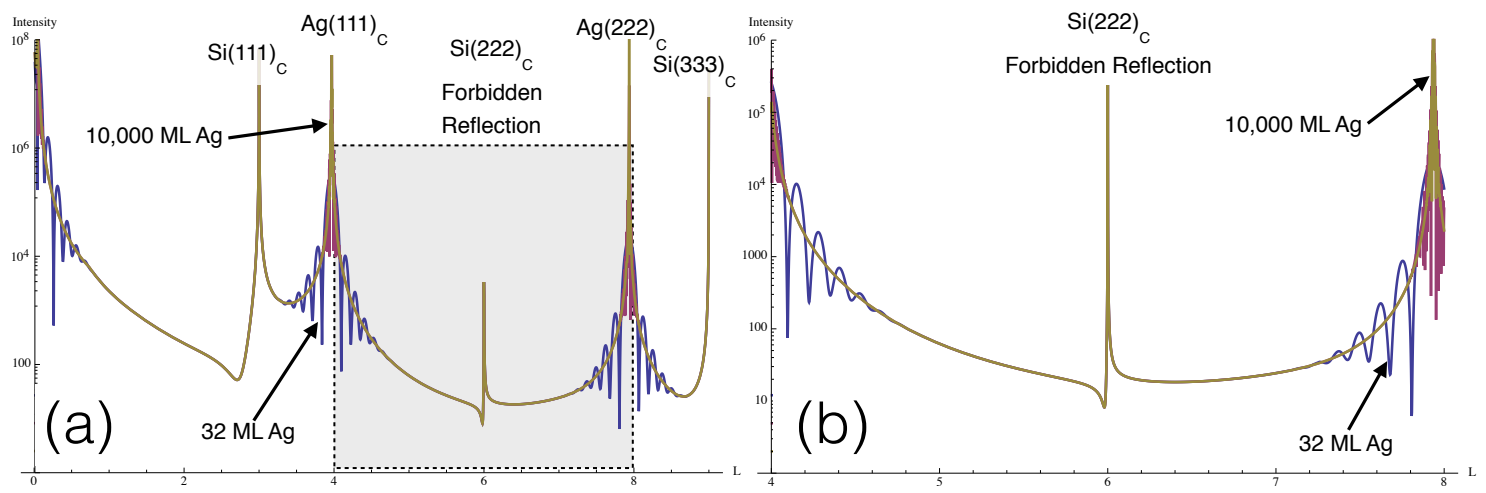

Figure 7.14: Calculated X-ray specular reflectivity curves for (blue curve) $32 \mathrm{ML}$ and (gold curve) 10,000 ML thick Ag films on $\mathrm{Si}(111)_{C} 7 \times 7$. (b) is a magnified view of the shaded region in (a) showing the region near the forbidden reflection. For thick films, changes to the $\mathrm{Ag}$ film thickness does not alter the reflectivity around the $\mathrm{Si}(222)_{C}$ forbidden reflection $\left(L_{H}=6\right)$.

the base of the forbidden reflection. Although, our calculations in Fig. 7.14 show that increasing the Ag film thickness by three orders of magnitude dose not further increase the intensity at the base of the $\mathrm{Si}(222)_{C}$ forbidden reflection. Rather, the increased film thickness dampens the Ag oscillations and increases the intensity of the Ag Bragg peaks.

Increases in intensity at the base of the forbidden Bragg reflection can be achieved by substituting $\mathrm{Ag}$ with an element that has a greater scattering cross section, i.e. greater $Z$. Care must be taken when selecting a candidate and one must take into account the lattice constant of the substitute material and select one that does not introduce a Bragg peak near the $\operatorname{Si}(222)_{C}$ forbidden reflection. In this situation the amplitude from the Bragg peak would complicate the interference with the $\mathrm{Si}(222)_{C}$ forbidden reflection. Rather, it is desirable for fitting the $\operatorname{Si}(222)_{C}$ forbidden reflection to have a thick film that makes the scattering near $L_{H}=6$ less sensitive to the Si substrate morphology as well as to the details of the film. Calculated curves in Fig. 7.15 demonstrate that when the substrate roughness is large the intensity drops off pre- 


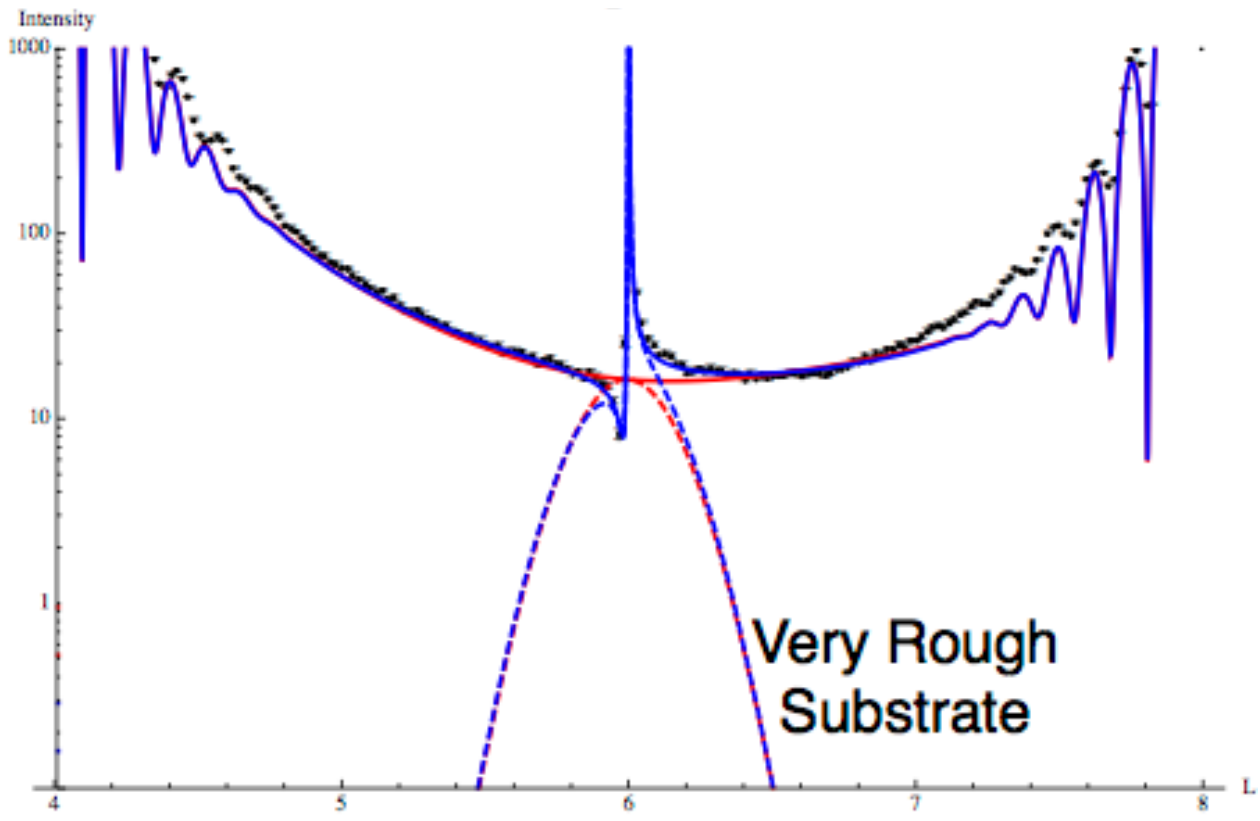

Figure 7.15: Calculated X-ray specular reflectivity curves for the thick Ag film on $\mathrm{Si}(111)_{C} 7 \times 7$ with different $\mathrm{Si}$ substrate roughness. The $\mathrm{Si}(222)_{C}$ forbidden reflection is at $L_{H}=6$. The best-fit curve is plotted with the solid blue curve. Keeping all other parameters fixed, the dashed blue curve is calculated for a substrate that is two orders of magnitude rougher than the roughness determined for the best-fit curve. The characteristic edge and cusp of the forbidden reflection remain even though the substrate is very rough.

cipitously between the Si Bragg peaks, yet the scattering near the forbidden Bragg reflection is preserved, including the characteristic edge and cusp, in the presence of a surface film.

The interplanar spacing, $d_{111}$, for the majority of elements, in there most common crystal structure can be found in Table A.1. The location of the $(111)_{C},(222)_{C}$, and $(333)_{C}$ Bragg peaks relative to the Si reciprocal lattice is also included in the table. A list of potential candidates for surface films on $\mathrm{Si}(111)_{C}$ that would provide an enhancement to the scattering is provided in Table A.2. From a practical standpoint Au would be a favorable substitute to Ag, where the intensity from a sufficiently thick $\mathrm{Au}$ is estimated to be three times greater than with Ag. Furthermore, the use of $\mathrm{Au}$ is also favorable because it is relatively chemically inert. 
Measuring and extracting a continuous electron density distribution is not limited to Si. Other crystals can be examined in using the method developed and demonstrated in this study. The continuous electron density distribution can be extracted from between the Bragg peaks along the CTR, as long as the surface is sufficiently smooth on the atomic scale. Centrosymmetric atoms can also be examined. In this case, $\gamma(\vec{Q})$, from Eq. (5.31) would only include $\operatorname{Re}(\gamma(\vec{Q}))$. Whereas, with other non-centrosymmetric atoms $\gamma(\vec{Q})$ would involve both real and imaginary parts, as in the case of Si. The phase can be extracted by two potential methods. The phase can be determined from the interference between the forbidden Bragg reflection and the CTR, as we demonstrated above. Though the adjacent cusp are only limited to crystals with non-centrosymmetric atoms such as Ge, $\alpha$-Tin, $\mathrm{C}$, and low $\Delta \mathrm{Z}$ compounds such as GaAs. In contrast to mono-atomic diamond crystal structures, in noncentrosymmetric compounds the spherical charge density distribution is not entirely canceled at the pseudo-forbidden reflections. Therefore low $\Delta \mathrm{Z}$ non-centrosymmetric compounds are advantageous for extracting the phase from the interference between the pseudo-forbidden Bragg reflection and the CTR. Otherwise the anisotropic component will be overwhelmed by the non-extinguished spherical charge density contribution. Secondly, destructive interference can be artificially introduce with a surface film. This method as was demonstrated above, where the destructive interference between the $\mathrm{Ag}$ and $\mathrm{Si}(111)_{C}$ Bragg peaks significantly enhanced the sensitivity to the anisotropic charge density distribution near $\mathrm{L}_{H} \approx 2.5$. Table A.1 can be used to identify a suitable film to deposit on a substrate. Utilizing the interference between the scattering from the surface and the bulk significantly expands the experimental capabilities for determining an electron density distribution.

In this study we have demonstrated experimentally that Si valence electron density distribution information can be attained using X-ray specular reflectivity from 
$\operatorname{Si}(111)_{C} 7 \times 7$ both with and without Ag films. With special care in dealing with the additional geometric complexities that are involved with in-plane CTR geometries one would be able to extract information about the charge density distribution along other directions as well. As seen in Fig. 5.10 and Fig. 7.4, the forbidden reflections along other CTRs would be sensitive to the anharmonic thermal vibrations of the core. Therefore at elevated temperatures, the sign of the anisotropic charge density along the rod could be determined with forbidden reflections that are very weak at room temperature.

In conclusion, using the unique abilities of the CTR technique to determine the bulk and surface structures simultaneously we have been able to determine - for the first time - the direction and radial density distribution of the covalent bond in Si. Using X-ray specular reflectivity we show that a novel interference effect permits one to determine the sign of the phase and therefore the direction of the anisotropic charge density. We demonstrate that the effect is robust by also verifying the effect on samples with different surface structures, including samples with thin and thick $\mathrm{Ag}$ films. Additionally, we show that non-spherical charge density distribution information can be extracted from CTR, which also allows for more accurate determination of the surface structure. The new capability to extract the charge density distribution information in-between the Bragg peaks and along different directions has the potential to be a valuable tool for expanding the understanding of charge density structure around an atom. 


\section{Appendix A}

\section{Lattice Matching}

\section{A.1 Lattice Matching}

The $(111)_{C}$ interplanar spacing, $d_{111}$, is shown in Table A.1 along with the location of the $(\text { nnn })_{C}$ Bragg peaks relative to the $\mathrm{Si}(111)_{C}$ reciprocal lattice units. $a, b, c$ and $\alpha, \beta, \gamma$ are the lengths and angles, respectively, of the real-space unit cell. The lattice parameters and crystal structures were compiled using MathematicA's[91] built-in function "LatticeData".

The interplanar spacing, $d_{h k l}$, for the orthorhombic system is [92]

$$
\frac{1}{d_{h k l}^{2}}=\frac{h^{2}}{a^{2}}+\frac{k^{2}}{b^{2}}+\frac{l^{2}}{c^{2}},
$$

where $h, k, l$ are the Miller indices. For the hexagonal system[69]

$$
\frac{1}{d_{h k l}^{2}}=\frac{4}{3}\left(\frac{h^{2}+h k+k^{2}}{a^{2}}\right)+\frac{l^{2}}{c^{2}} .
$$


For the rhombohedral we have,[69]

$$
\frac{1}{d_{h k l}^{2}}=\frac{\left(h^{2}+k^{2}+l^{2}\right) \sin (\alpha)^{2}+2(h k+k l+h l) \cos (\alpha)^{2}-\cos (\alpha)}{a^{2}\left(1-3 \cos (\alpha)^{2}+2 \cos (\alpha)^{3}\right)} .
$$

The spacing for the monoclinic is determined by[69]

$$
\frac{1}{d_{h k l}^{2}}=\frac{1}{\sin (\beta)^{2}}\left(\frac{h^{2}}{a^{2}}+\frac{k^{2} \sin (\beta)^{2}}{b^{2}}+\frac{l^{2}}{c^{2}}-\frac{2 h l \cos (\beta)}{a c}\right) .
$$

The lattice plane spacing for triclinic is determined by[92]

$$
\frac{1}{d_{h k l}^{2}}=\frac{1}{V^{2}}\left(S_{11} h^{2}+S_{22} k^{2}+S_{33} l^{2}+2\left(S_{12} h k+S_{23} k l+S_{13} h l\right)\right),
$$

where $V$ is unit cell volume and

$$
\begin{aligned}
& V^{2}=a^{2} b^{2} c^{2}\left(1+2 \cos (\alpha) \cos (\beta) \cos (\gamma)-\cos (\alpha)^{2}-\cos (\beta)^{2}-\cos (\gamma)^{2}\right) \\
& S_{11}=b^{2} c^{2} \sin (\alpha)^{2} \\
& S_{22}=a^{2} c^{2} \sin (\beta)^{2} \\
& S_{33}=a^{2} b^{2} \sin (\gamma)^{2} \\
& S_{12}=a b c^{2}(\cos (\alpha) \cos (\beta)-\cos (\gamma)) \\
& S_{23}=a^{2} b c(\cos (\beta) \cos (\gamma)-\cos (\alpha)), \\
& S_{13}=a b^{2} c(\cos (\gamma) \cos (\alpha)-\cos (\beta)) .
\end{aligned}
$$




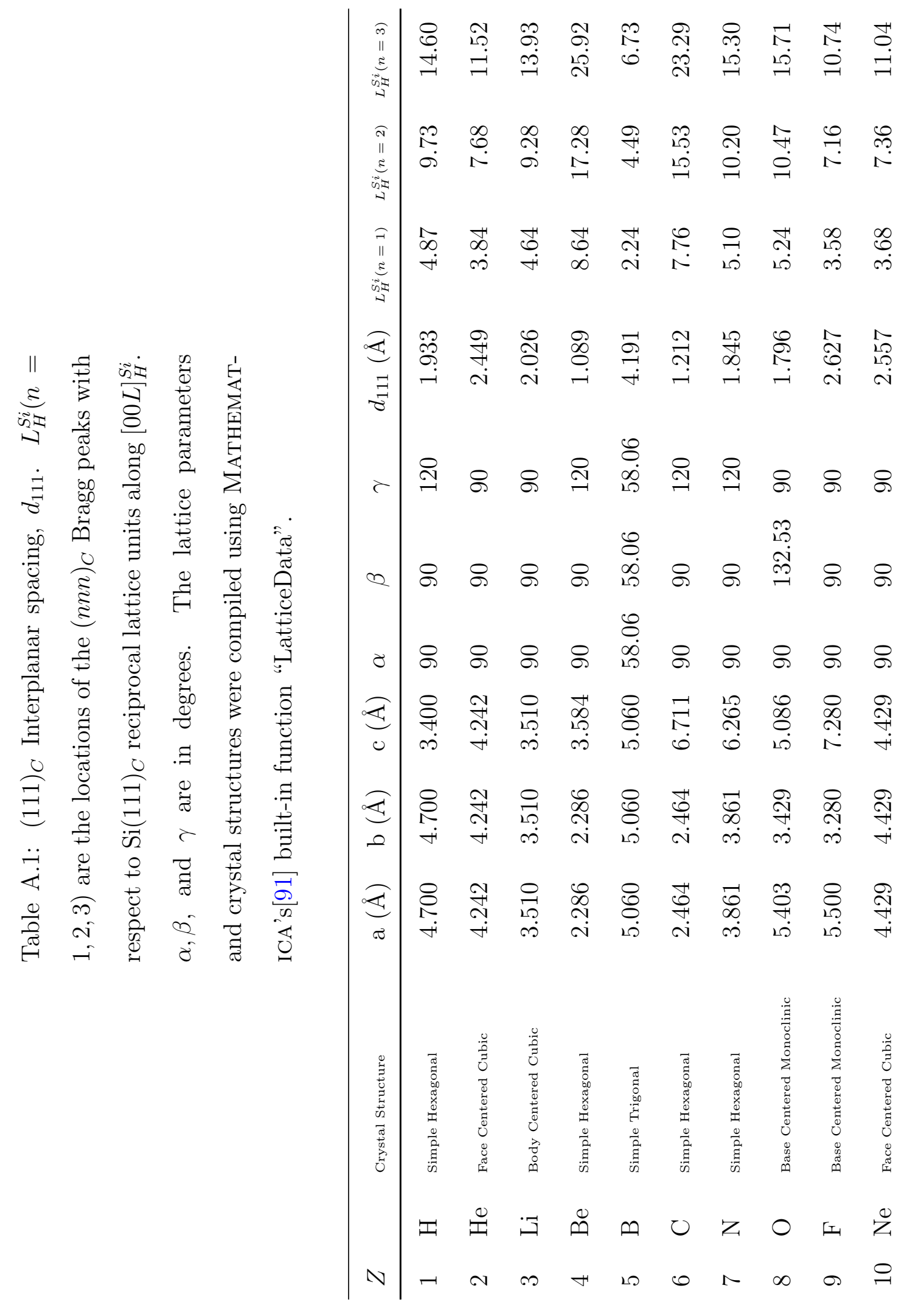




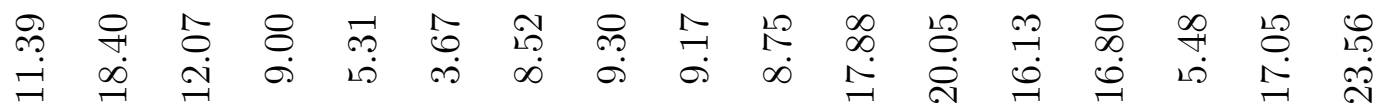

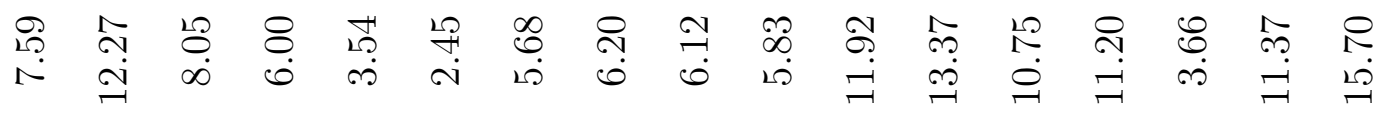

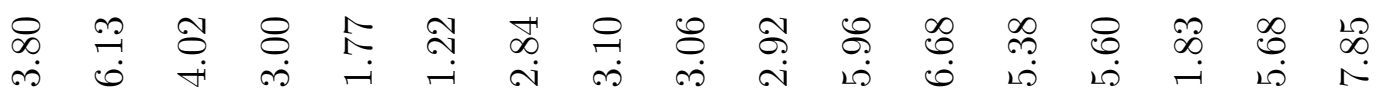

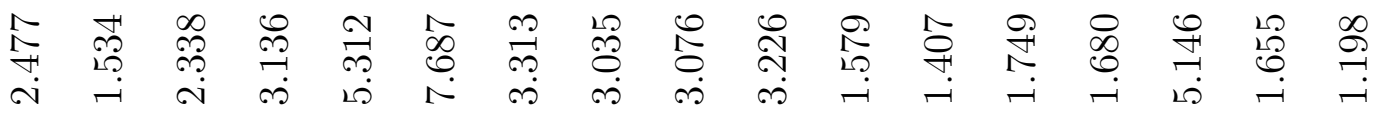

৪ ㅇ ஃ ৪

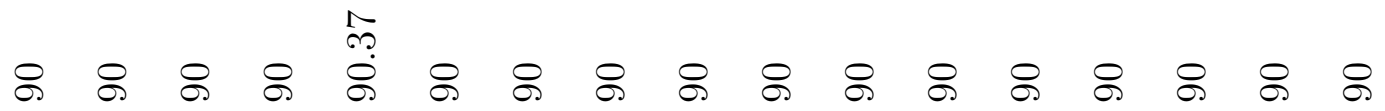

৪ ৪ ৪

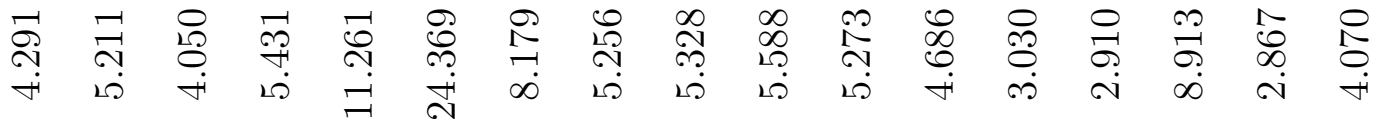

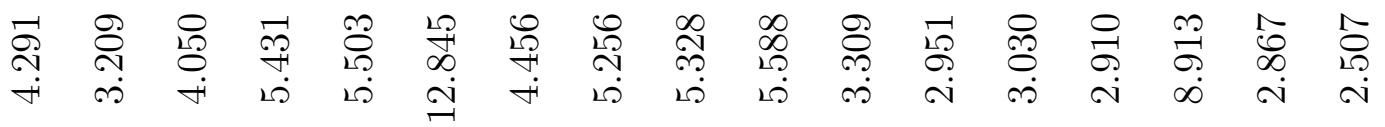

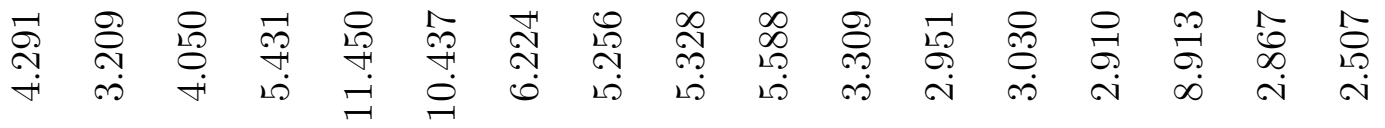

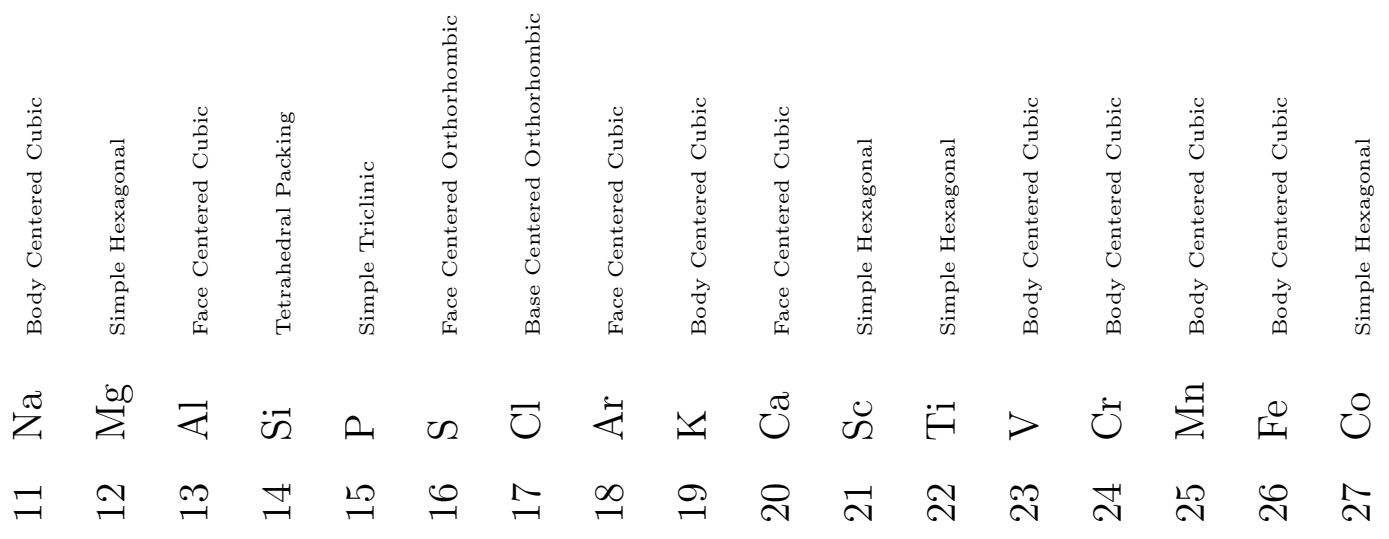




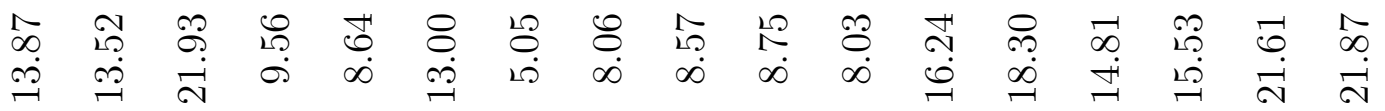

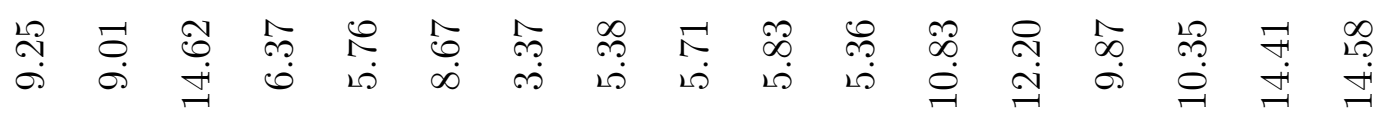

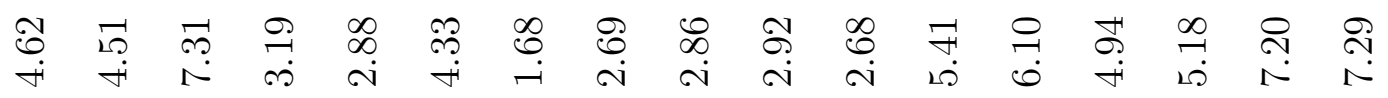

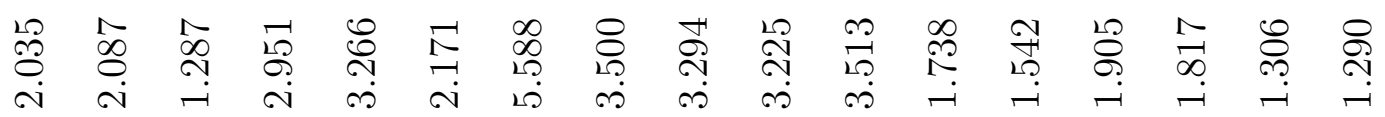

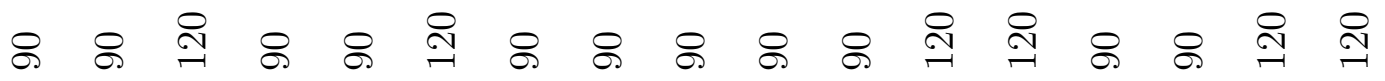

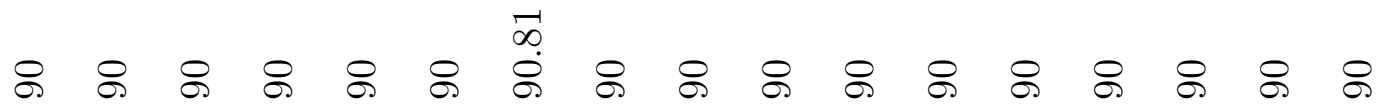

๑ \& \& \& \& \& \& \& \& \& \& \& \& \& \& \&

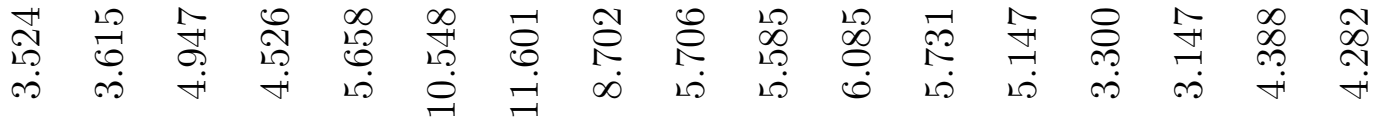

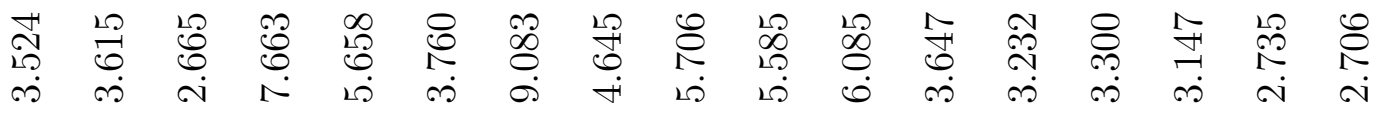

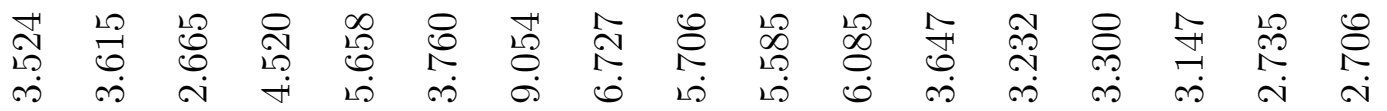

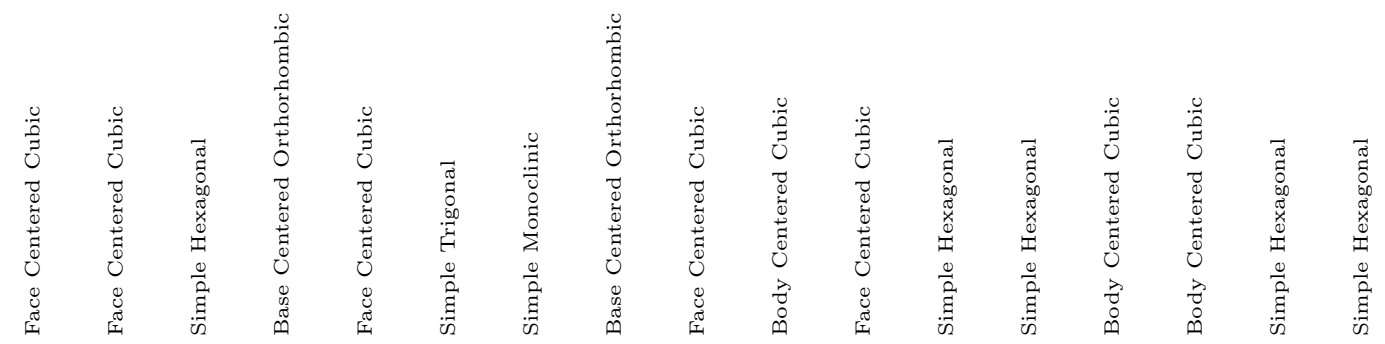

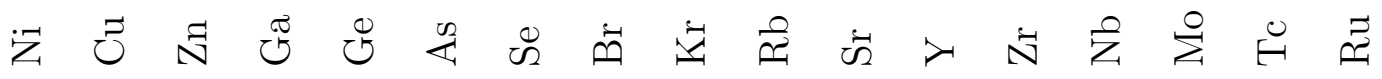

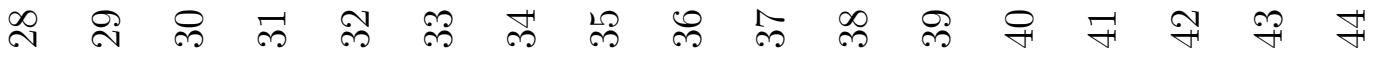




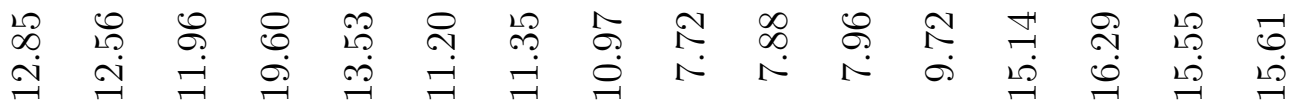

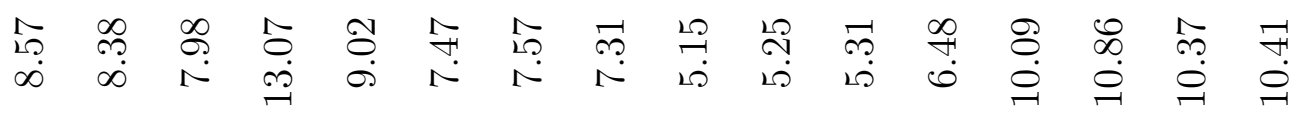

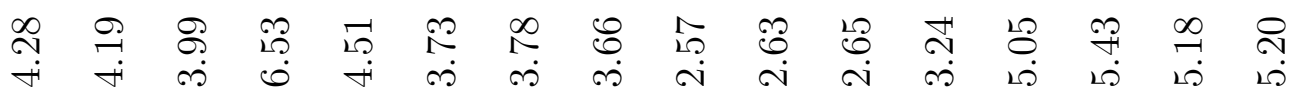

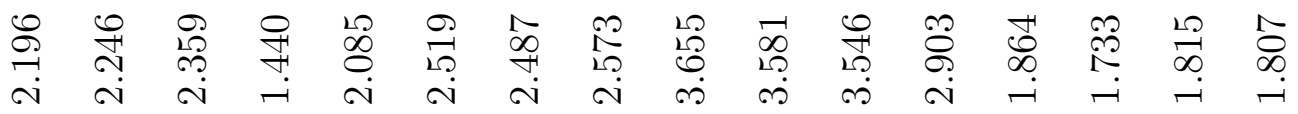

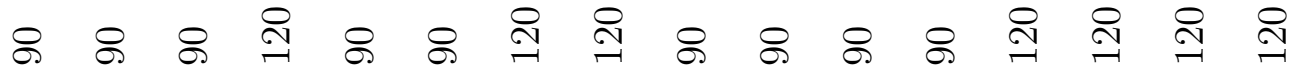

ஃ ஃ ஃ ஃ ஃ ஃ ஃ ஃ ஃ ஃ ஃ ஃ ஃ

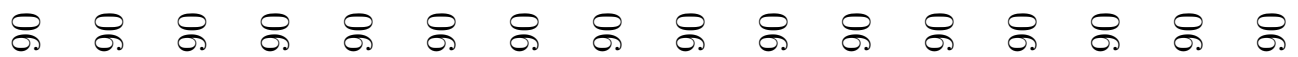

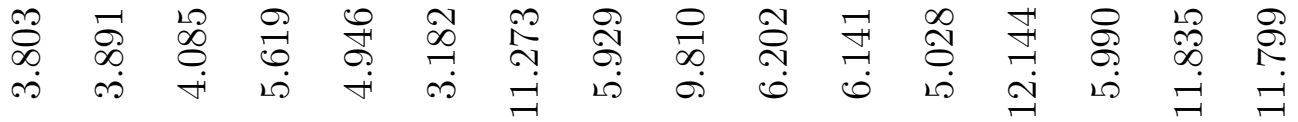

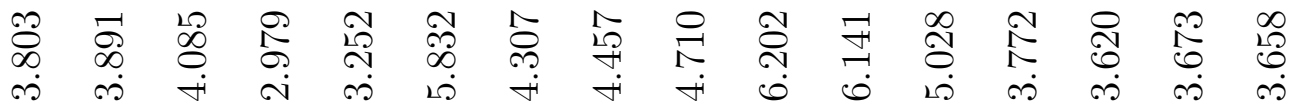

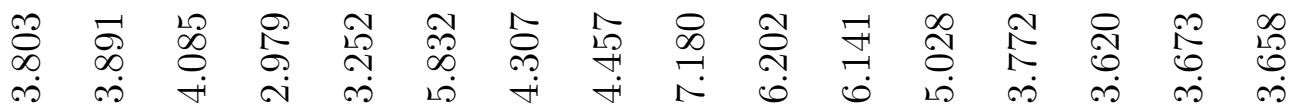

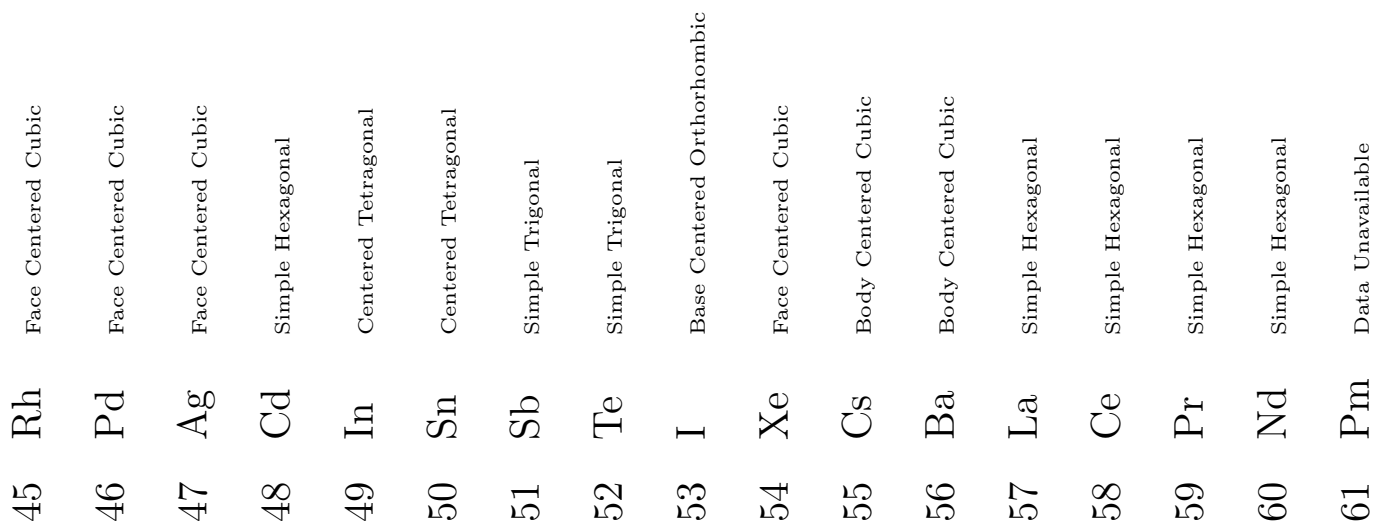




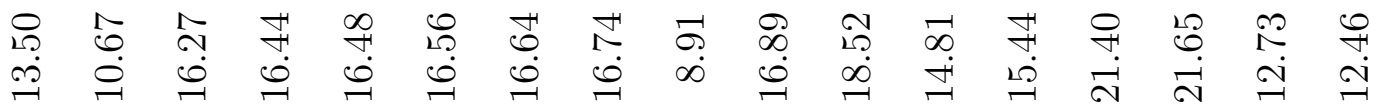

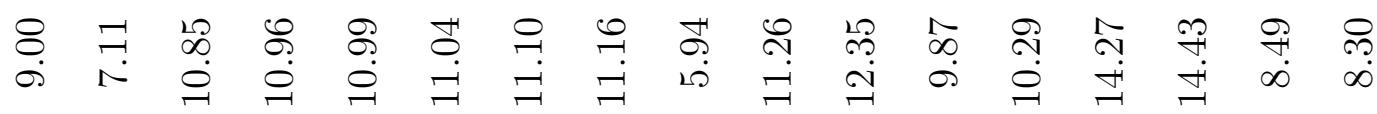

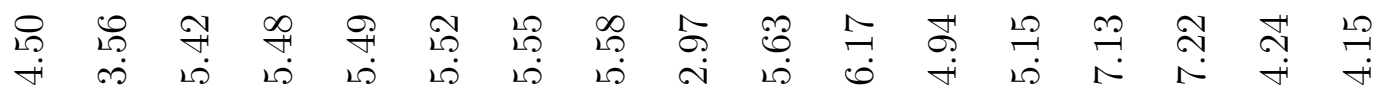

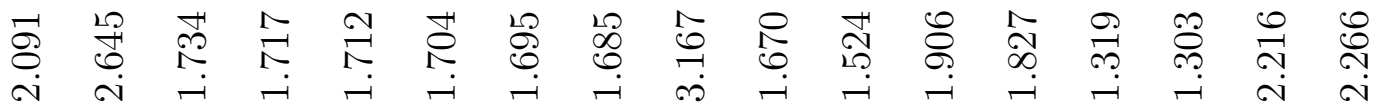

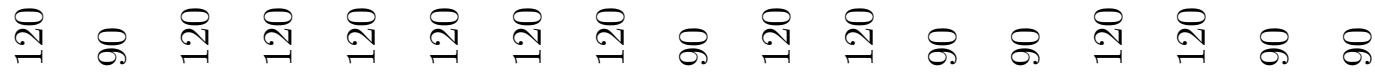

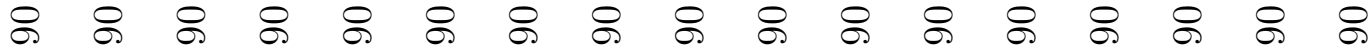

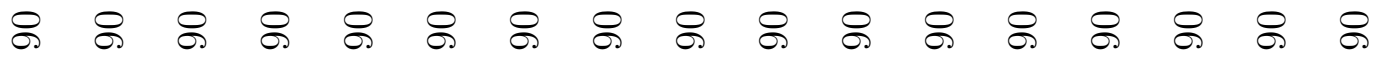

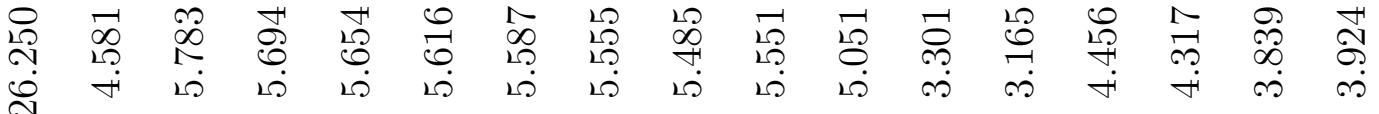

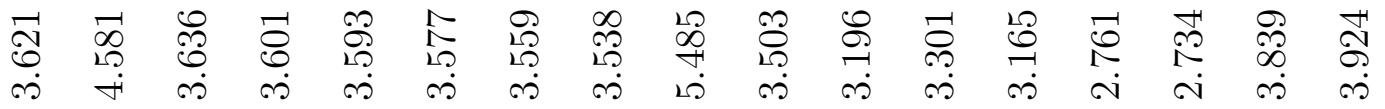

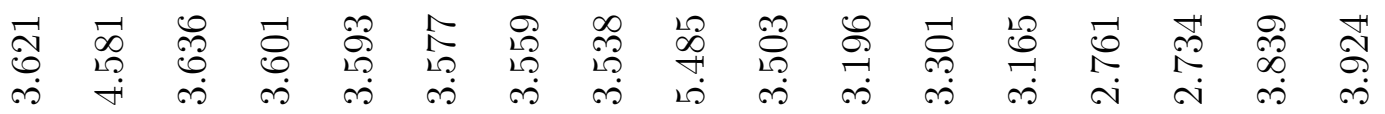

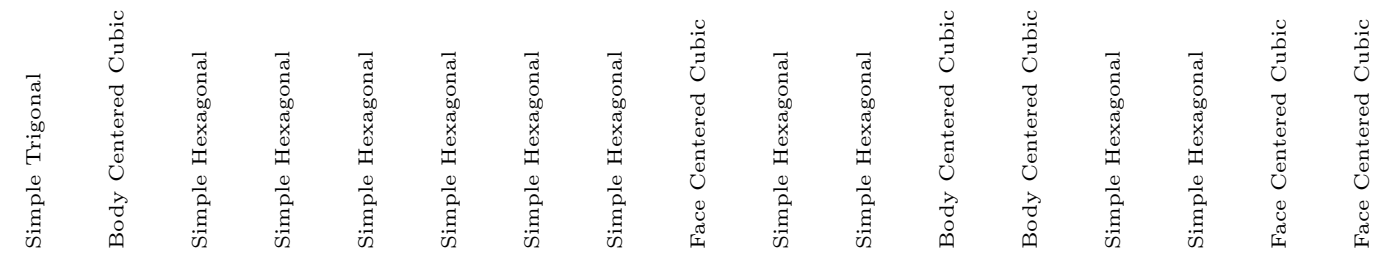

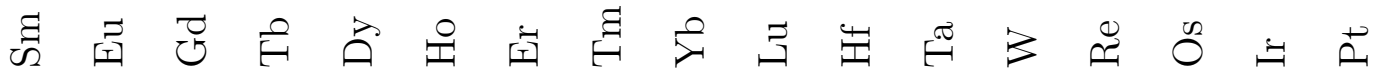
กู 


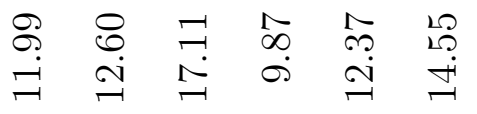

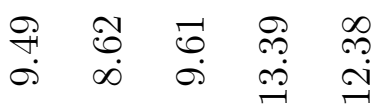

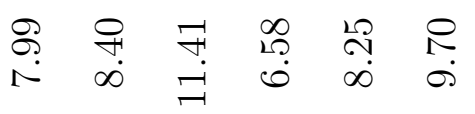

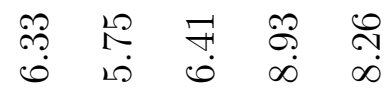

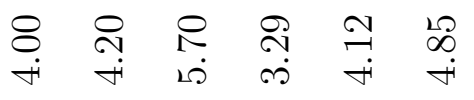

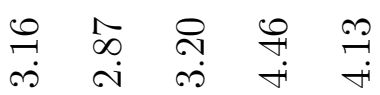

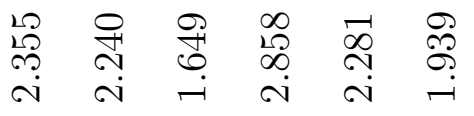

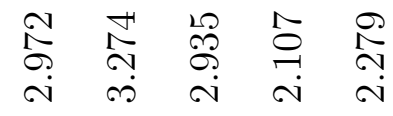

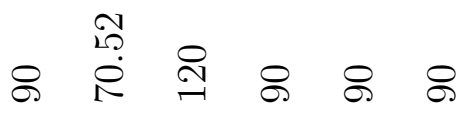

$$
\begin{aligned}
& 88888
\end{aligned}
$$

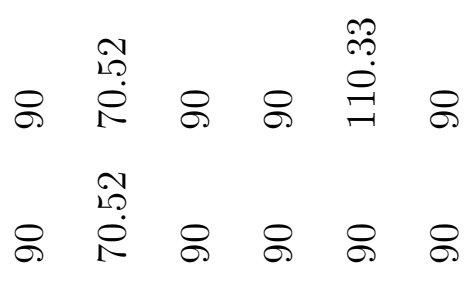

$$
\begin{aligned}
& \text { ৪ \& \& \& }
\end{aligned}
$$

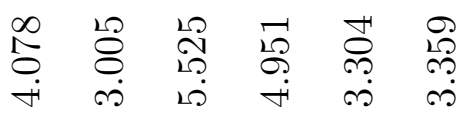

$$
\begin{aligned}
& \text { \& \& \& \& }
\end{aligned}
$$

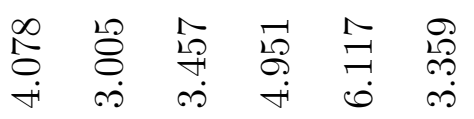

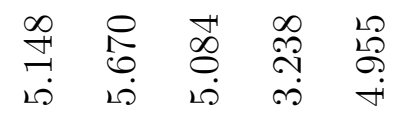

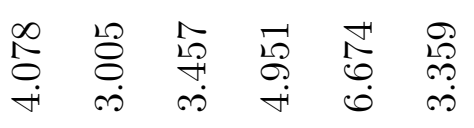

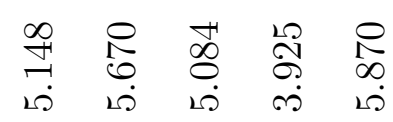

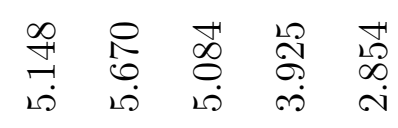

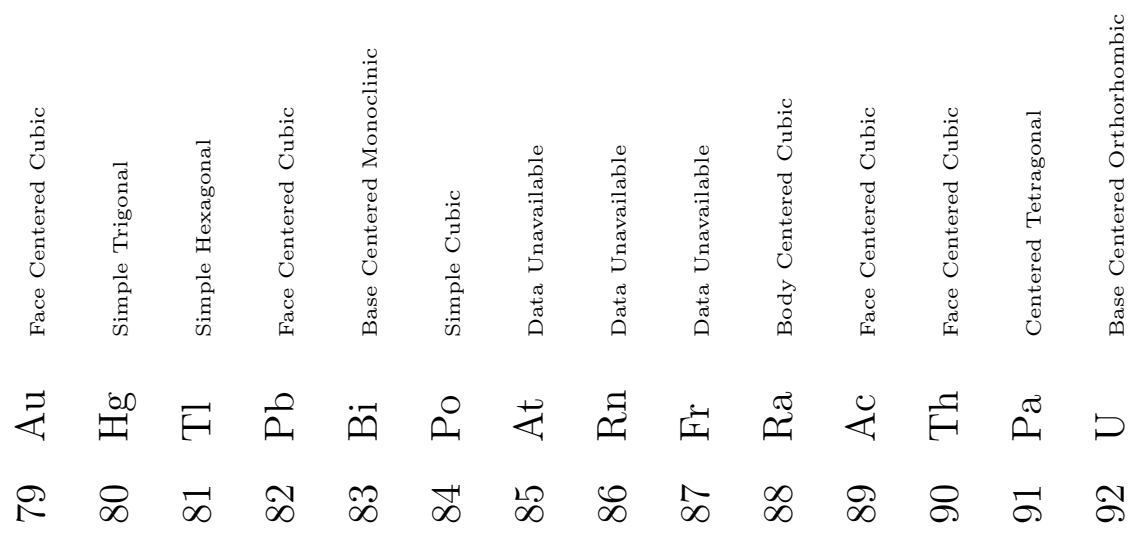


Table A.2: Candidates for surface films on $\mathrm{Si}(111)_{C}$, selected from Table A.1. $L_{H}^{S i}(n=1,2,1.5)$ are the locations of the $(n n n)_{C}$ Bragg peaks $(n=1,2)$ and the anti-Bragg $(n=1.5)$ with respect to $\mathrm{Si}(111)_{C}$ reciprocal lattice units along $[00 L]_{H}^{S i}$.

\begin{tabular}{lllccccr}
\hline$Z$ & & Crystal Structure & $d_{111}(\AA)$ & $L_{H}^{S i}(n=1)$ & $L_{H}^{S i}(n=2)$ & $L_{H}^{S i}(n=1.5)$ & $I_{\text {increase }}$ \\
\hline \hline 33 & $\mathrm{As}$ & Simple Trigonal & 2.171 & 4.33 & 8.67 & 6.50 & 5.6 \\
45 & $\mathrm{Rh}$ & Face Centered Cubic & 2.196 & 4.28 & 8.57 & 6.43 & 10.3 \\
77 & $\mathrm{Ir}$ & Face Centered Cubic & 2.216 & 4.24 & 8.49 & 6.37 & 30.3 \\
80 & $\mathrm{Hg}$ & Simple Trigonal & 2.240 & 4.20 & 8.40 & 6.30 & 32.7 \\
46 & $\mathrm{Pd}$ & Face Centered Cubic & 2.246 & 4.19 & 8.38 & 6.28 & 10.8 \\
78 & $\mathrm{Pt}$ & Face Centered Cubic & 2.266 & 4.15 & 8.30 & 6.23 & 31.0 \\
92 & $\mathrm{U}$ & Base Centered Orthorhombic & 2.279 & 4.13 & 8.26 & 6.19 & 43.2 \\
83 & $\mathrm{Bi}$ & Base Centered Monoclinic & 2.281 & 4.12 & 8.25 & 6.19 & 35.1 \\
79 & $\mathrm{Au}$ & Face Centered Cubic & 2.355 & 4.00 & 7.99 & 5.99 & 31.8 \\
47 & $\mathrm{Ag}$ & Face Centered Cubic & 2.359 & 3.99 & 7.98 & 5.98 & 11.3 \\
51 & $\mathrm{Sb}$ & Simple Trigonal & 2.487 & 3.78 & 7.57 & 5.67 & 13.3 \\
50 & $\mathrm{Sn}$ & Centered Tetragonal & 2.519 & 3.73 & 7.47 & 5.60 & 12.8 \\
\hline
\end{tabular}

\section{A.2 Potential Film Candidates on $\mathrm{Si}(111)_{C}$}

Table A.2 includes candidate elements that could be deposited on the $\mathrm{Si}(111)_{C}$ surface to enhance the specular reflectivity intensity near the $\mathrm{Si}(222)_{C}$ forbidden Bragg reflection. Si wafers are commercially available with $(100)_{C},(110)_{C}$, and $(111)_{C}$ terminations. Though the Si forbidden Bragg reflections do not appear in the specular reflectivity from the $(100)_{C}$ and $(110)_{C}$ terminated wafers because at least one of the Miller indices are zero along the specular direction. Therefore film candidates for $(100)_{C}$ and $(110)_{C}$ terminated wafers are not included here. The candidates were selected from Table A.1 base on the following criteria: The anti-Bragg location, halfway between the $(111)_{C}$ and $(222)_{C}$ Bragg peaks, did not exceed $L_{H}^{S i}=6 \pm 0.5$. The intensity is enhanced with the surface film, $I_{\text {increase }}>1$, where the enhancement is estimated by $I_{\text {increase }}=\frac{Z_{f i l m}{ }^{2}}{Z_{S i}{ }^{2}}$. 


\section{Bibliography}

[1] Josh Goldberger et al. "Silicon Vertically Integrated Nanowire Field Effect Transistors". In: Nano Letters 6.5 (May 2006), pp. 973-977. DOI: 10.1021/ nl060166j. URL: http://pubs.acs.org/doi/abs/10.1021/nl060166j.

[2] Volker Schmidt et al. "Realization of a Silicon Nanowire Vertical Surround-Gate Field-Effect Transistor". In: Small 2.1 (Jan. 2006), pp. 85-88. DOI: 10.1002/ smll.200500181. URL: http://doi.wiley.com/10.1002/smll.200500181.

[3] Bozhi Tian et al. "Coaxial silicon nanowires as solar cells and nanoelectronic power sources". In: Nature 449.7164 (Oct. 2007), pp. 885-889. DOI: 10.1038/nature06181. URL: http://www . nature.com/doifinder/10.1038/ nature06181.

[4] Francesco Priolo et al. "Silicon nanostructures for photonics and photovoltaics". In: Nature Nanotechnology 9.1 (Jan. 2014), pp. 19-32. DOI: 10.1038/nnano. 2013.271. URL: http://www . nature. com/doifinder/10.1038/nnano. 2013. 271.

[5] Candace K. Chan et al. "High-performance lithium battery anodes using silicon nanowires". In: Nature Nanotechnology 3.1 (Jan. 2008), pp. 31-35. DOI: 10. 1038/nnano. 2007.411. URL: http://www . nature.com/doifinder/10.1038/ nnano. 2007.411 .

[6] Kuiqing Peng et al. "Aligned Single-Crystalline Si Nanowire Arrays for Photovoltaic Applications". In: Small 1.11 (Nov. 2005), pp. 1062-1067. DOI: 10.1002/ smll.200500137. URL: http://doi.wiley.com/10.1002/smll.200500137.

[7] Kui-Qing Peng et al. "Silicon nanowires for advanced energy conversion and storage". In: Nano Today 8.1 (Feb. 2013), pp. 75-97. DOI: 10.1016/j . nantod. 2012 . 12 .009. URL: http://linkinghub. elsevier.com/retrieve/pii / S1748013212001466. 
[8] Akram I. Boukai et al. "Silicon nanowires as efficient thermoelectric materials". In: Nature 451.7175 (Jan. 2008), pp. 168-171. DOI: $10.1038 /$ nature06458. URL: http://www. nature.com/doifinder/10.1038/nature06458.

[9] Allon I. Hochbaum et al. "Enhanced thermoelectric performance of rough silicon nanowires". In: Nature 451.7175 (Jan. 2008), pp. 163-167. DOI: 10. 1038 / nature06381. URL: http: / / www . nature com/doifinder / 10 . 1038/nature06381.

[10] Roey Elnathan et al. "Engineering vertically aligned semiconductor nanowire arrays for applications in the life sciences". In: Nano Today 9.2 (Apr. 2014), pp. 172-196. DOI: $10.1016 / \mathrm{j}$. nantod . 2014.04 .001 . URL: http : //linkinghub.elsevier.com/retrieve/pii/S1748013214000462.

[11] M. Lajvardi et al. "Structural and optical properties of silicon nanowires synthesized by Ag-assisted chemical etching". In: Materials Science in Semiconductor Processing 40 (Dec. 2015), pp. 556-563. DOI: $10.1016 / \mathrm{j}$. mssp . 2015 . 07 .032. URL: http://linkinghub. elsevier.com/retrieve/pii/ S1369800115300688.

[12] Zhipeng Huang et al. "Metal-Assisted Chemical Etching of Silicon: A Review: In memory of Prof. Ulrich Gösele". In: Advanced Materials 23.2 (Jan. 2011), pp. 285-308. DOI: 10.1002/adma. 201001784. URL: http://doi.wiley.com/ 10.1002/adma. 201001784 .

[13] J. B. Roberto and B. W. Batterman. "Anharmonicity and the Temperature Dependence of the Forbidden (222) Reflection in Silicon". In: Physical Review B 2.8 (1970), p. 3220. URL: http://journals . aps .org/prb/abstract/10. 1103/PhysRevB.2.3220.

[14] David Keating et al. "Anharmonicity and the temperature dependence of the forbidden (222) reflection in silicon". In: Physical Review Letters 27.6 (1971), p. 320. URL: https://journals .aps .org/prl/abstract/10.1103/ PhysRevLett.27.320.

[15] P. Trucano and B. W. Batterman. "Bonding-Electron Distributions, Anharmonicity, and the Temperature Dependence of the Forbidden Si (442) Reflection". In: Physical Review B 6.10 (1972), p. 3659. URL: http://journals .aps . org/prb/abstract/10.1103/PhysRevB.6.3659.

[16] J. B. Hastings and B. W. Batterman. "High-order anharmonic forbidden neutron reflections in silicon". In: Physical Review B 12.12 (1975), p. 5580. URL: https://journals.aps.org/prb/abstract/10.1103/PhysRevB.12.5580. 
[17] J. Z. Tischler and B. W. Batterman. "Determination of magnitude, phase, and temperature dependence of forbidden reflections in silicon and germanium". In: Physical Review B 30.12 (1984), p. 7060. URL: http://journals . aps .org/ prb/abstract/10.1103/PhysRevB.30.7060.

[18] D. Mills and B. W. Batterman. "Synchrotron-radiation measurements of forbidden reflections in silicon and germanium". In: Physical Review B 22.6 (1980), p. 2887. URL: http://journals.aps .org/prb/abstract/10.1103/PhysRevB . 22.2887.

[19] Jesse W. Kremenak et al. "Insight on the silver catalyst distribution during silicon nanowire array formation: an X-ray reflectivity study". In: Nanoscale 9.48 (2017), pp. 19073-19085. DOI: 10.1039/C7NR05676A. URL: http://xlink. rsc . org $/$ ?DOI=C7NR05676A.

[20] Jesse W. Kremenak and Danielle V. Langdon. "Back cover". In: Nanoscale 9.48 (Dec. 2017), pp. 19416-19416. DOI: 10 . 1039/C7NR90272G. URL: http: //pubs.rsc.org/en/content/articlelanding/2017/nr/c7nr90272g.

[21] Kuiqing Peng et al. "Motility of Metal Nanoparticles in Silicon and Induced Anisotropic Silicon Etching". In: Advanced Functional Materials 18.19 (Oct. 2008), pp. 3026-3035. DOI: 10 . 1002 /adfm . 200800371. URL: http: / / doi . wiley.com/10.1002/adfm. 200800371.

[22] Miao-Rong Zhang, Feng-Xia Wang, and Ge-Bo Pan. "Metal-assisted photochemical etching of gallium nitride using electrodeposited noble metal nanoparticles as catalysts". In: Electrochemistry Communications 76 (Mar. 2017), pp. 59-62. DOI: 10 . 1016/j . elecom . 2017 . 01 .021. URL: http : //linkinghub.elsevier.com/retrieve/pii/S1388248117300309.

[23] Yunwon Song and Jungwoo Oh. "Fabrication of three-dimensional GaAs antireflective structures by metal-assisted chemical etching". In: Solar Energy Materials and Solar Cells 144 (Jan. 2016), pp. 159-164. DOI: 10.1016/j.solmat. 2015 . 08 .034. URL: http : / / linkinghub . elsevier . com/retrieve/pii / S0927024815004286.

[24] Fatima Toor et al. "Metal assisted catalyzed etched (MACE) black Si: optics and device physics". In: Nanoscale 8.34 (2016), pp. 15448-15466. DOI: 10.1039/ C6NR04506E. URL: http://xlink.rsc.org/?DOI=C6NR04506E.

[25] Hee Han, Zhipeng Huang, and Woo Lee. "Metal-assisted chemical etching of silicon and nanotechnology applications". In: Nano Today 9.3 (June 2014), pp. 271-304. DOI: 10 . 1016/ j . nantod . 2014 . 04 .013. URL: http : //linkinghub.elsevier.com/retrieve/pii/S1748013214000589. 
[26] Matt DeJarld et al. "Formation of High Aspect Ratio GaAs Nanostructures with Metal-Assisted Chemical Etching". In: Nano Letters 11.12 (Dec. 2011), pp. 5259-5263. DOI: 10.1021/n1202708d. URL: http://pubs . acs .org/doi/ $\mathrm{abs} / 10.1021 / \mathrm{nl} 202708 \mathrm{~d}$.

[27] Michael P. Marder. Condensed Matter Physics. 2nd ed. Hoboken: Wiley, 2010. ISBN: 978-0-470-61798-4. URL: https : / / books . google . com / books ? id = ijloadAt4BQC.

[28] Gilles Renaud, Rémi Lazzari, and Frédéric Leroy. "Probing surface and interface morphology with Grazing Incidence Small Angle X-Ray Scattering". In: Surface Science Reports 64.8 (Aug. 2009), pp. 255-380. DOI: $10.1016 /$ j. surfrep. 2009 . 07 .002. URL: http : / / linkinghub . elsevier . com/retrieve/pii / S0167572909000399.

[29] Sanjay K. Srivastava et al. "Excellent antireflection properties of vertical silicon nanowire arrays". In: Solar Energy Materials and Solar Cells 94.9 (Sept. 2010), pp. 1506-1511. DOI: 10 .1016/j . solmat . 2010.02 .033. URL: http: //linkinghub.elsevier.com/retrieve/pii/S0927024810000863.

[30] Kuiqing Peng et al. "Dendrite-Assisted Growth of Silicon Nanowires in Electroless Metal Deposition". In: Advanced Functional Materials 13.2 (2003), pp. 127132 .

[31] T. Qiu et al. "From Si nanotubes to nanowires: Synthesis, characterization, and self-assembly". In: Journal of Crystal Growth 277.1-4 (Apr. 2005), pp. 143148. DOI: 10 .1016/j . jcrysgro . 2005.01 .095. URL: http://linkinghub . elsevier.com/retrieve/pii/S0022024805001302.

[32] T. Qiu et al. "Intergrowth mechanism of silicon nanowires and silver dendrites". In: Journal of Electronic Materials 35.10 (2006), pp. 1879-1884.

[33] Hui Fang et al. "Silver catalysis in the fabrication of silicon nanowire arrays". In: Nanotechnology 17.15 (Aug. 2006), pp. 3768-3774. DOI: 10 . 1088/09574484/17/15/026. URL: http://stacks. iop.org/0957-4484/17/i=15/a= 026?key=crossref . c6736f c1ca5a444d9ba007e8c57ba974.

[34] Shinji Yae et al. "Formation of porous silicon by metal particle enhanced chemical etching in HF solution and its application for efficient solar cells". In: Electrochemistry Communications 5.8 (Aug. 2003), pp. 632-636. DOI: 10.1016/S13882481 (03)00146-2. URL: http://linkinghub.elsevier.com/retrieve/pii/ S1388248103001462.

[35] C. Chartier, S. Bastide, and C. Lévy-Clément. "Metal-assisted chemical etching of silicon in HF-H2O2". In: Electrochimica Acta 53.17 (July 2008), pp. 5509- 
5516. DOI: $10.1016 / \mathrm{j}$.electacta.2008.03.009. URL: http://linkinghub . elsevier.com/retrieve/pii/S0013468608003782.

[36] K. Q. Peng et al. "Fabrication of Single-Crystalline Silicon Nanowires by Scratching a Silicon Surface with Catalytic Metal Particles". In: Advanced Functional Materials 16.3 (Feb. 2006), pp. 387-394. DOI: 10.1002/adfm. 200500392. URL: http://doi.wiley.com/10.1002/adfm. 200500392.

[37] Winston Chern et al. "Nonlithographic Patterning and Metal-Assisted Chemical Etching for Manufacturing of Tunable Light-Emitting Silicon Nanowire Arrays". In: Nano Letters 10.5 (May 2010), pp. 1582-1588. DOI: 10 . 1021 / nl903841a. URL: http://pubs.acs.org/doi/abs/10.1021/n1903841a.

[38] P. Scherrer. "Bestimmung der Grösse und der inneren Struktur von Kolloidteilchen mittels Röntgenstrahlen". In: Nachrichten von der Gesellschaft der Wissenschaften zu Göttingen 26 (1918), pp. 98-100.

[39] Ilya M Lifshitz and Vitaly V Slyozov. "The kinetics of precipitation from supersaturated solid solutions". In: Journal of physics and chemistry of solids 19.1-2 (1961), pp. 35-50.

[40] Dinesh Kumar et al. "Room temperature growth of wafer-scale silicon nanowire arrays and their Raman characteristics". In: Journal of Nanoparticle Research 12.6 (Aug. 2010), pp. 2267-2276. DOI: 10.1007/s11051-009-9795-7. URL: http://link.springer.com/10.1007/s11051-009-9795-7.

[41] P. F. Miceli. "X-ray reflectivity from heteroepitaxial layers". In: Semiconductor Interfaces, Microstructures and Devices: Properties and Applications. Institute of Physics: Bristol. Ed. by Z. C. Feng. Bristol: IOP Publishing, 1993, pp. 87114.

[42] J. Als-Nielsen and Des McMorrow. Elements of Modern X-ray Physics. 2nd ed. Hoboken: Wiley, 2011. ISBN: 978-0-470-97395-0 978-0-470-97394-3.

[43] Y. Yoneda. "Anomalous Surface Reflection of X Rays". In: Physical Review 131.5 (Sept. 1963), pp. 2010-2013. DOI: 10 . 1103/PhysRev . 131 . 2010. URL: http://link.aps.org/doi/10.1103/PhysRev.131.2010.

[44] S. K. Sinha et al. "X-ray and neutron scattering from rough surfaces". In: Physical Review B 38.4 (1988), p. 2297. URL: http: / / journals . aps . org / prb/abstract/10.1103/PhysRevB.38.2297.

[45] P.A. Kienzle et al. REFLPAK. 2000. URL: http: / / www . ncnr . nist . gov / reflpak. 
[46] L. G. Parratt. "Surface Studies of Solids by Total Reflection of X-Rays". In: Physical Review 95.2 (July 1954), pp. 359-369. DOI: 10.1103/PhysRev. 95.359. URL: http://link.aps.org/doi/10.1103/PhysRev.95.359.

[47] Xing Zhong et al. "Unveiling the Formation Pathway of Single Crystalline Porous Silicon Nanowires". In: ACS Applied Materials \& Interfaces 3.2 (Feb. 2011), pp. 261-270. DOI: 10.1021/am1009056. URL: http://pubs .acs .org/ doi/abs/10.1021/am1009056.

[48] Xiaoge Gregory Zhang. Electrochemistry of Silicon and Its Oxide. New York: Kluwer Academic Publishers, 2001. ISBN: 0-306-47921-4. URL: http: //www . ebrary.com/.

[49] V.G. Tsirelson and R.P. Ozerov. Electron Density and Bonding in Crystals: Principles, Theory and X-ray Diffraction Experiments in Solid State Physics and Chemistry. Taylor \& Francis, 1996. ISBN: 978-0-7503-0284-5. URL: https: //books.google.com/books?id=ud_DmLcBXZ4C.

[50] Piero Macchi et al. "Modelling the experimental electron density: only the synergy of various approaches can tackle the new challenges". In: IUCrJ 2.4 (July 2015), pp. 441-451. DOI: 10 . 1107 / S2052252515007538. URL: http : //scripts.iucr.org/cgi-bin/paper?S2052252515007538.

[51] P. Coppens. X-Ray Charge Densities and Chemical Bonding. International Union of Crystallography Texts on Crystallography. International Union of Crystallography, 1997. ISBN: 978-0-19-535694-6. URL: https://books .google. com. jm/books? id=2CjYv3EuRxOC.

[52] Moshe Deutsch. "Electronic charge distribution in crystalline silicon". In: Physical Review B 45.2 (1992), p. 646. URL: http://journals . aps . org/prb/ abstract/10.1103/PhysRevB.45.646.

[53] Carlo Gatti and Piero Macchi, eds. Modern Charge-Density Analysis. DOI: 10.1007/978-90-481-3836-4. Dordrecht: Springer Netherlands, 2012. ISBN: 97890-481-3835-7 978-90-481-3836-4. URL: http://link. springer.com/10.1007/ 978-90-481-3836-4.

[54] B. Dawson. "The covalent bond in silicon". In: Proceedings of the Royal Society of London A: Mathematical, Physical and Engineering Sciences. Vol. 298. The Royal Society, 1967, pp. 379-394. URL: http : / / rspa . royalsocietypublishing.org/content/298/1455/379. short.

[55] B. Dawson. "The Covalent Bond in Diamond". In: Proceedings of the Royal Society A: Mathematical, Physical and Engineering Sciences 298.1454 (May 
1967), pp. 264-288. DOI: 10 . 1098/rspa . 1967 .0103. URL: http : / / rspa . royalsocietypublishing.org/cgi/doi/10.1098/rspa.1967.0103.

[56] B. Dawson and P. L. Sanger. "X-ray studies of covalent bonding in diamond and silicon". In: 301 (1967), pp. 195-209. URL: http : / / rspa . royalsocietypublishing.org/content/301/1465/195. short.

[57] Z. W. Lu, Alex Zunger, and Moshe Deutsch. "Electronic charge distribution in crystalline diamond, silicon, and germanium". In: Physical Review B 47.15 (1993), p. 9385. URL: https://journals .aps.org/prb/abstract/10.1103/ PhysRevB . 47.9385.

[58] Fred C. Von der Lage and H. A. Bethe. "A method for obtaining electronic eigenfunctions and eigenvalues in solids with an application to sodium". In: Physical Review 71.9 (1947), p. 612. URL: https://journals.aps.org/pr/ abstract/10.1103/PhysRev.71.612.

[59] M. Fehlmann. "Bonding Electron Distribution in Silicon". In: Journal of the Physical Society of Japan 47.1 (July 1979), pp. 225-231. DOI: 10.1143/JPSJ . 47.225. URL: https://doi.org/10.1143/JPSJ .47.225.

[60] B. Dawson. "A General Structure Factor Formalism for Interpreting Accurate X-ray and Neutron Diffraction Data". In: Proceedings of the Royal Society A: Mathematical, Physical and Engineering Sciences 298.1454 (May 1967), pp. 255-263. DOI: 10 . $1098 /$ rspa . 1967 .0102. URL: http://rspa . royalsocietypublishing.org/cgi/doi/10.1098/rspa.1967.0102.

[61] B. Dawson. "X-ray scattering and covalent bonding in germanium". In: Proceedings of the Royal Society of London A: Mathematical, Physical and Engineering Sciences. Vol. 298. The Royal Society, 1967, pp. 395-401. URL: http: //rspa.royalsocietypublishing.org/content/298/1455/395. short.

[62] B. Dawson and B. T. M. Willis. "Anharmonic Vibration and Forbidden Reflexions in Silicon and Germanium". In: Proceedings of the Royal Society A: Mathematical, Physical and Engineering Sciences 298.1454 (May 1967), pp. 307-315. DOI: 10 . 1098/rspa . 1967 .0105. URL: http : / / rspa . royalsocietypublishing.org/cgi/doi/10.1098/rspa.1967.0105.

[63] B. Dawson, A. C. Hurley, and V. W. Maslen. "Anharmonic Vibration in Fluorite Structures". In: Proceedings of the Royal Society of London. Series A, Mathematical and Physical Sciences 298.1454 (1967), pp. 289-306. URL: http: //www. jstor .org/stable/2416064. 
[64] U. W. Arndt et al. "X-rays". In: International Tables for Crystallography Volume C: Mathematical, physical and chemical tables. Springer, 2006, pp. 191258.

[65] P. Coppens, Z. Su, and P. J. Becker. "Analysis of charge and spin densities". In: International Tables for Crystallography Volume C: Mathematical, physical and chemical tables. Springer, 2006, pp. 713-734.

[66] J. B. Roberto, B. W. Batterman, and D. T. Keating. "Diffraction studies of the (222) reflection in Ge and Si: Anharmonicity and the bonding electron". In: Physical Review B 9.6 (1974), p. 2590. URL: http://journals.aps.org/prb/ abstract/10.1103/PhysRevB.9.2590.

[67] P. Coppens. "The Structure Factor". In: International Tables for Crystallography. 2nd ed. Vol. Volume B: Reciprocal space. 2010, pp. 10-23. ISBN: 978-14020-8205-4. DOI: 10.1107/97809553602060000759.

[68] P. J. E. Aldred and M. Hart. "The electron distribution in Silicon. I. Experiment". In: Proceedings of the Royal Society of London A: Mathematical, Physical and Engineering Sciences 332 (1973), pp. 223-238. URL: http: //rspa . royalsocietypublishing. org/content/332/1589/223. short.

[69] B.E. Warren. X-ray Diffraction. Addison-Wesley series in metallurgy and materials engineering. Dover Publications, 1969. ISBN: 978-0-486-66317-3. URL: https://books .google.com/books?id=wfLBhAbEYAsC.

[70] W. H. Bragg. "The intensity of X-ray reflection by diamond". In: Proceedings of the Physical Society of London 33.1 (1920), p. 304. URL: http://iopscience. iop.org/1478-7814/33/1/331.

[71] Charles Kittel. Introduction to Solid State Physics. 8th. John Wiley \& Sons, Inc, 2005. ISBN: 978-0-471-41526-8.

[72] Yiyao Chen. "In-situ x-ray scattering studies of Ag nano-structures". Thesis. University of Missouri-Columbia, 2016. URL: https : //mospace . umsystem . edu/xmlui/handle/10355/60410.

[73] Niels Bindzus et al. "Experimental determination of core electron deformation in diamond". In: Acta Crystallographica Section A Foundations and Advances 70.1 (Jan. 2014), pp. 39-48. DOI: 10 . $1107 /$ S2053273313026600. URL: http: //scripts.iucr.org/cgi-bin/paper?S2053273313026600.

[74] Mads R. V. Jørgensen et al. "Contemporary X-ray electron-density studies using synchrotron radiation". In: IUCrJ 1.5 (Sept. 2014), pp. 267-280. DOI: 10.1107/ 
S2052252514018570. URL: http : / / scripts . iucr . org / cgi-bin/paper? S2052252514018570.

[75] Nanna Wahlberg et al. "Powder X-ray Diffraction Electron Density of Cubic Boron Nitride". In: The Journal of Physical Chemistry C 119.11 (Mar. 2015), pp. 6164-6173. DOI: 10.1021/jp511985d. URL: http://pubs . acs .org/doi/ 10.1021/jp511985d.

[76] A. Fischer et al. "Experimental and Theoretical Charge Density Studies at Subatomic Resolution". In: The Journal of Physical Chemistry A 115.45 (Nov. 2011), pp. 13061-13071. DOI: 10.1021/jp2050405. URL: http://pubs . acs . org/doi/abs/10.1021/jp2050405.

[77] David Keating et al. "Forbidden (222) Neutron Reflection in Silicon: Anharmonicity and the Bonding Electrons". In: Physical Review B 4.8 (1971), p. 2472.

[78] Isao Fujimoto. "Temperature and pressure dependence of the Si (222) forbidden reflection and the vibration of the bonding charge". In: Physical Review B 9.2 (1974), p. 591. URL: http://journals . aps .org/prb/abstract/10 . 1103/ PhysRevB.9.591.

[79] N. K. Hansen and P. Coppens. "Testing aspherical atom refinements on smallmolecule data sets". In: Acta Crystallographica Section A 34.6 (Nov. 1978), pp. 909-921. DOI: 10 . 1107 / S0567739478001886. URL: http : / / scripts . iucr.org/cgi-bin/paper?S0567739478001886.

[80] S. R. Andrews and R. A. Cowley. "Scattering of x-rays from crystal surfaces". In: Journal of Physics C: Solid State Physics 18 (1985), pp. 6427-6439.

[81] I. K. Robinson. "Crystal truncation rods and surface roughness". In: Physical Review B 33.6 (1986), pp. 3830-3836.

[82] I. K. Robinson. "X-ray Crystallography of Surfaces and Interfaces". In: Acta Crystallographica Section A: Foundations of Crystallography 54.6 (1998), pp. 772-778. URL: http : / / scripts . iucr . org/cgi-bin/paper? S0108767398008125.

[83] I. K. Robinson and D. J. Tweet. "Surface x-ray diffraction". In: Reports on Progress in Physics 55 (1992), pp. 599-651.

[84] I. K. Robinson. "Surface Crystallography". In: Handbook on Synchrotron Radiation. Ed. by G. Brown and D. E. Moncton. Vol. 3. Elsevier Science Publishers B. V., 1991. 
[85] M. W. Gramlich et al. "Nonequilibrium behavior of the $\mathrm{Pb}$ wetting layer on Si(111)7x7". In: Physical Review B 84.7 (Aug. 2011). DoI: 10.1103/PhysRevB . 84.075433. URL: http://link.aps.org/doi/10.1103/PhysRevB.84.075433.

[86] Yiyao Chen et al. "In situ x-ray scattering study of Ag island growth on $\mathrm{Si}(111)$ 7x7". In: Physical Review B 94.4 (July 28, 2016). DOI: 10.1103/PhysRevB. 94. 045437. URL: http://link.aps.org/doi/10.1103/PhysRevB.94.045437.

[87] W. C. Elliott et al. "Orientation dependence of homoepitaxy: An in situ xray scattering study of Ag". In: Physica B: Condensed Matter 221.14 (1996), pp. $65-69$.

[88] I.K. Robinson and E. Vlieg. "X-ray reflectivity study of the $\mathrm{Si}(111) 7 \mathrm{x} 7$ surface". In: Surface Science 261 (1992), pp. 123-128.

[89] Michael W. R. Gramlich. "X-ray diffraction studies on the $\mathrm{Pb} / \mathrm{Si}(111)$ system". Thesis. University of Missouri-Columbia, 2011. URL: https://mospace. umsystem.edu/xmlui/handle/10355/14402.

[90] R. W. Alkire, W. B. Yelon, and J. R. Schneider. "Determination of the absolute structure factor for the forbidden (222) reflection in silicon using $0.12-\AA \gamma$ rays". In: Physical Review B 26.6 (1982), p. 3097. URL: http://journals.aps.org/ prb/abstract/10.1103/PhysRevB.26.3097.

[91] Wolfram Research, Inc. Mathematica, Version 11.3. Champaign, IL, 2018.

[92] A. M Glazer, Morgan \& Claypool Publishers, and Institute of Physics (Great Britain). A journey into reciprocal space: a crystallographer's perspective. OCLC: 1012426609. 2017. ISBN: 978-1-68174-621-0 978-1-68174-623-4 978-168174-620-3. URL: http://iopscience.iop.org/book/978-1-6817-4621-0. 


\section{VITA}

Jesse Wade Kremenak was born in Seoul, South Korea. He grew up in Silver Spring, Maryland, where he attended James Hubert Blake High School. In 2008, he received his Bachelor of Science degree in Physics and Art from Ursinus College, Collegeville, Pennsylvania, and was awarded the Thomas J. Watson Fellowship. He received a Masters of Science degree in Physics from the University of Missouri (MU) in 2013. During his graduate study Jesse has received several honors and awards, which include; the TA Choice Award, Missouri Student Association; Harry E. Hammond Award, Department of Physics and Astronomy; Superior Graduate Student Award, MU Graduate Student Association; inducted into the Rollins Society, University of Missouri; the National Association of Graduate-Professional Students (NAGPS) Award of Excellence in Advocacy; 2014 Outstanding Board Member Award, NAGPS; the Sandra K. Abell Science Education Award, MU Office of Graduate Studies; and the International Recognition Award, Missouri International Student

Council. In May 2018, Jesse will receive a Ph.D. in Physics from the University of Missouri. 